\title{
La violence au préscolaire et au primaire
}

Les défis et les enjeux de la collaboration entre l'école et les parents

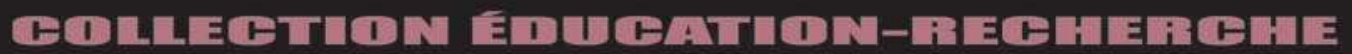

MARYSE PAQUIN

MARIE DROLET

Avec la collaboration de Rachel Hasan

Préface de

ÉGIDE ROYER

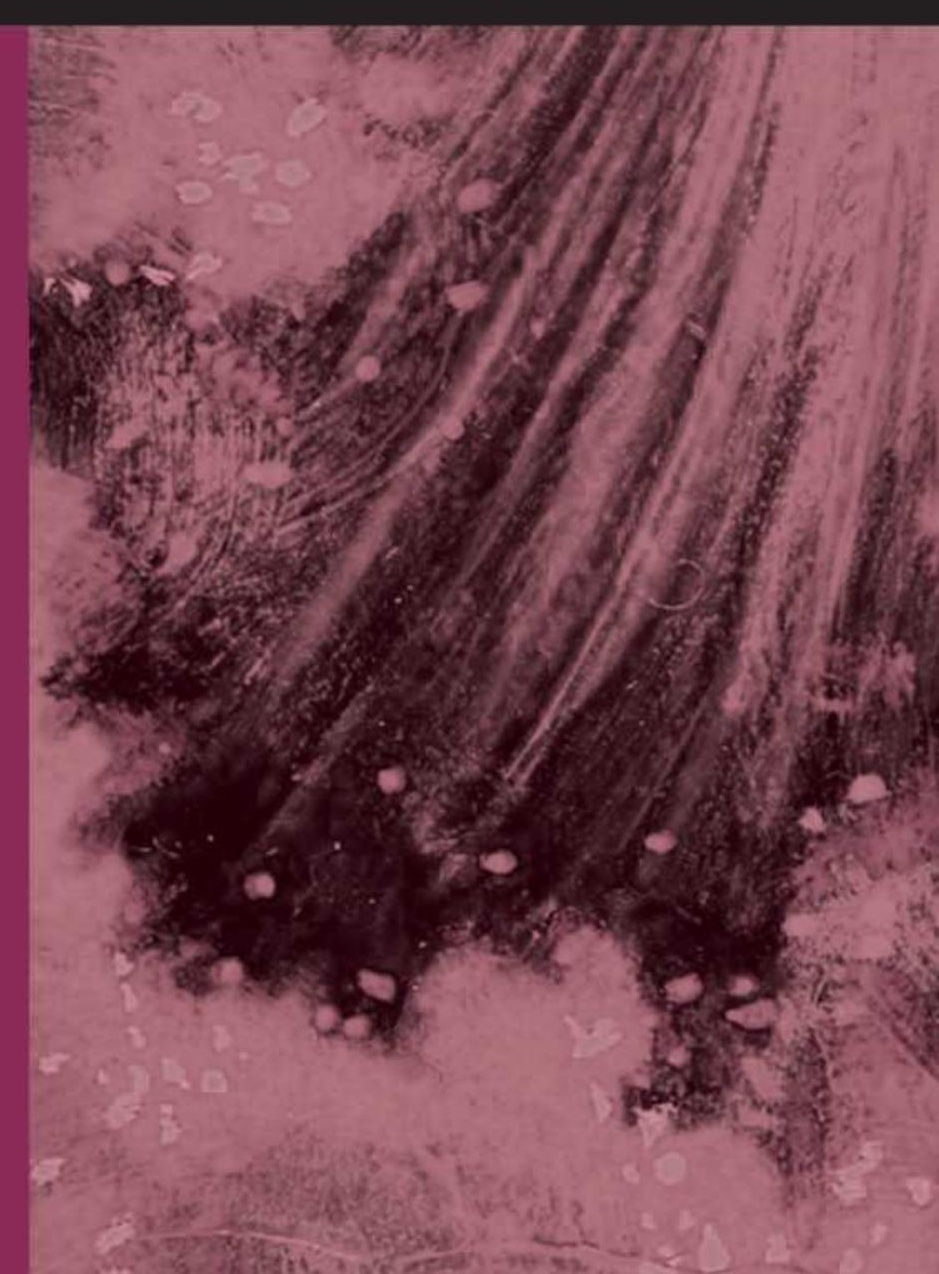

de l'Université du Québec 




\section{La violence au préscolaire et au primaire}


PRESSES DE L'UNIVERSITÉ DU QUÉBEC

Le Delta I, 2875, boulevard Laurier, bureau 450

Sainte-Foy (Québec) G1V 2M2

Téléphone: (418) 657-4399 • Télécopieur: (418) 657-2096

Courriel: puq@puq.ca·Internet : www.puq.ca

Diffusion / Distribution :

\section{CANADA et autres pays}

DisTRIBUTION DE LIVRES UNIVERS S.E.N.C.

845, rue Marie-Victorin, Saint-Nicolas (Québec) G7A 3S8

Téléphone: (418) 831-7474/1-800-859-7474 • Télécopieur: (418) 831-4021

FRANCE

AFPU-DIFFUSION

SoDIS

\section{SUISSE}

SERVIDIS SA

5, rue des Chaudronniers, $\mathrm{CH}-1211$ Genève 3, Suisse

Téléphone: 0229609525

Télécopieur: 0227763527

La Loi sur le droit d'auteur interdit la reproduction des œuvres sans autorisation des titulaires de droits. Or, la photocopie non autorisée - le «photocopillage »s'est généralisée, provoquant une baisse des ventes de livres et compromettant la rédaction et la production de nouveaux ouvrages par des professionnels. L'objet du logo apparaissant ci-contre est d'alerter le lecteur sur la menace que représente pour l'avenir de l'écrit le développement massif du «photocopillage ». 


\section{Collection ÉduCATION-RECHERCHE}

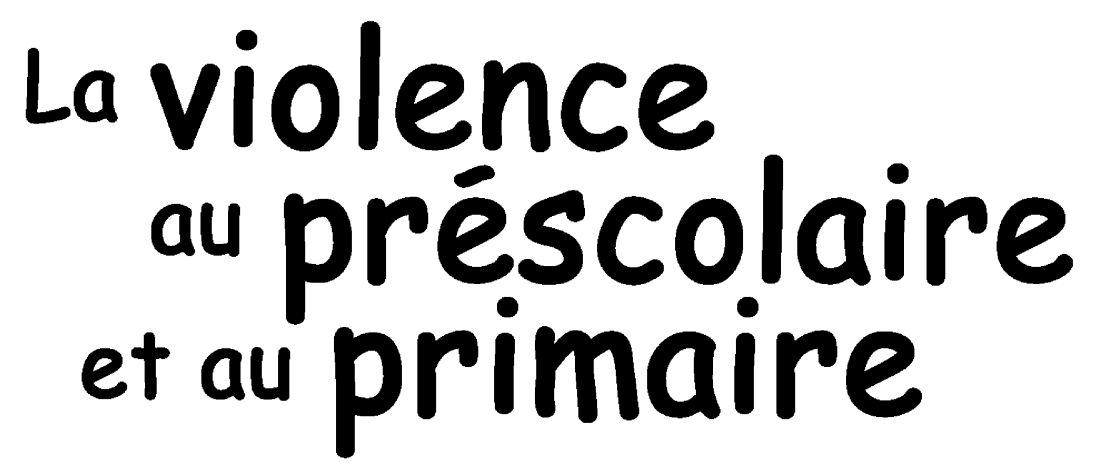

Les défis et les enjeux de la collaboration entre l'école et les parents

MARYSE PAQUIN et

MARIE DROLET

Avec la collaboration de

RACHEL HASAN

Préface de

ÉGIDE ROYER 
Paquin, Maryse, 1961-

La violence au préscolaire et au primaire: les défis et les enjeux de la collaboration entre l'école et les parents

(Collection Éducation-recherche; 19)

Comprend des réf. bibliogr.

ISBN 2-7605-1383-1

1. Violence dans les écoles - Ontario - Ottawa, Région d'. 2. Violence chez l'enfant - Ontario - Ottawa, Région d'. 3. Famille et école - Ontario - Ottawa, Région d'. 4. Éducation préscolaire - Participation des parents - Ontario Ottawa, Région d'. 5. Enseignement primaire - Participation des parents Ontario - Ottawa, Région d'. 6. Élèves - Suspension - Ontario - Ottawa, Région d'. I. Drolet, Marie, 1957- . II. Hasan, Rachel. III. Titre. IV. Collection.

Nous reconnaissons l'aide financière du gouvernement du Canada par l'entremise du Programme d'aide au développement de l'industrie de l'édition (PADIÉ) pour nos activités d'édition.

La publication de cet ouvrage a été rendue possible avec l'aide financière de la Société de développement des entreprises culturelles (SODEC).

Cet ouvrage a été publié grâce à une subvention de la Fédération canadienne des sciences humaines de concert avec le Progamme d'aide à l'édition savante, dont les fonds proviennent du Conseil de recherches en sciences humaines du Canada.

Révision linguistique : Louis Courteau

Mise en pages : INFO IOOO MOTS INC.

Couverture : RichaRd Hodgson

\section{PUQ 2006987654321}

Tous droits de reproduction, de traduction et d'adaptation réservés (C) 2006 Presses de l'Université du Québec

Dépôt légal - $1^{\text {er }}$ trimestre 2006

Bibliothèque nationale du Québec / Bibliothèque nationale du Canada

Imprimé au Canada 


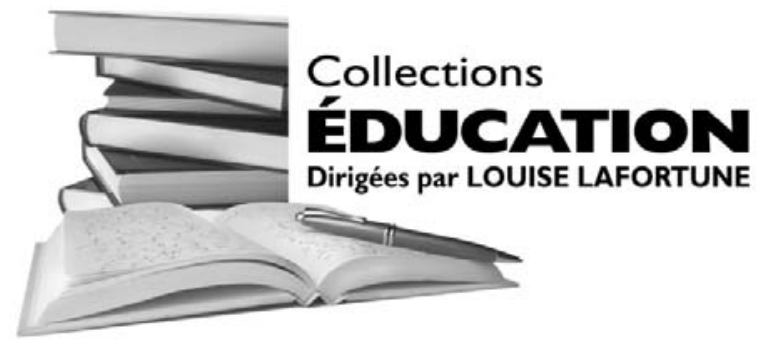

I es développements récents de la recherche en éducation Lont permis de susciter diverses réflexions pédagogiques et didactiques et de proposer plusieurs approches novatrices reconnues. Les nouveaux courants de recherche donnent lieu à un dynamisme et à une créativité dans le monde de l'éducation qui font en sorte que les préoccupations ne sont pas seulement orientées vers la recherche appliquée et fondamentale, mais aussi vers l'élaboration de moyens d'intervention pour le milieu scolaire.

Les Presses de l'Université du Québec, dans leur désir de tenir compte de ces intérêts diversifiés autant du milieu universitaire que du milieu scolaire, proposent deux nouvelles collections qui visent à rejoindre autant les personnes qui s'intéressent à la recherche (ÉduCATION-RECHERCHE) que celles qui développent des moyens d'intervention (ÉducAtionINTERVENTION).

Ces collections sont dirigées par madame Louise Lafortune, professeure au Département des sciences de l'éducation de I'Université du Québec à Trois-Rivières, qui, forte d'une grande expérience de publication et très active au sein des groupes de recherche et dans les milieux scolaires, leur apporte dynamisme et rigueur scientifique.

ÉdUCATION-ReCHERCHE et ÉdUCATION-INTERVENTION s'adressent aux personnes désireuses de mieux connaître les innovations en éducation qui leur permettront de faire des choix éclairés associés à la recherche et à la pédagogie. 



\section{$P R$ E F A C E}

Égide Royer, Ph. D., professeur, Faculté d'éducation, Université Laval codirecteur, Observatoire canadien pour la prévention de la violence à l'école 

Les conduites agressives de certains élèves du préscolaire et du primaire constituent une source de stress important, autant pour les intervenants scolaires que pour les jeunes eux-mêmes et leurs parents. Cette situation est présentement au premier rang des préoccupations des enseignants, qu'ils soient très expérimentés ou en début de carrière. Demandez, par exemple, à des enseignantes du préscolaire qui ont plus de vingt ans d'expérience de comparer les difficultés des élèves qu'elles vivaient dans les cinq premières années de leur carrière à celles de ceux qui fréquentent leur classe actuellement. La plupart de ces enseignantes vont témoigner qu'elles observent chaque semaine des incivilités et des comportements agressifs qui étaient relativement rares auparavant. Certaines de ces situations deviennent carrément dangereuses: agressions avec des ciseaux ou des crayons, crises de colère, fugues et menaces verbales envers les adultes, etc. Pour les enseignants, le fait d'avoir, comme c'est fréquemment le cas, un ou deux élèves aux comportements imprévisibles ou carrément incontrôlables en classe a un impact direct sur leur qualité de vie, sur celle des autres élèves de la classe, mais également sur la réussite éducative et l'insertion sociale de ces jeunes, de même que sur les relations entre l'école et leurs parents.

Les parents, de leur côté, ont parfois des difficultés à identifier les bonnes conduites à adopter pour prévenir et composer avec ce type de comportements lorsque leurs jeunes les manifestent à la maison. Être parent n'a jamais été facile et ne l'est pas davantage aujourd'hui. Ils sont souvent confrontés à des événements stressants (réorganisation familiale, chômage, violence conjugale, monoparentalité, etc.) qui viennent quelquefois perturber leurs pratiques éducatives. Les balises sur «le quand et le comment» intervenir, suite aux comportements violents de leur enfant, ne sont plus aussi claires qu'auparavant dans plusieurs familles. Doit-on le punir? Faut-il plutôt le faire réfléchir aux actes qu'il a posés? Serait-il mieux de l'ignorer? Devrait-on d'abord lui faire comprendre la portée de ses gestes? Cette ambivalence, voire cette insécurité quant à ce qui devrait être fait, explique en partie pourquoi on rencontre quelquefois des parents dont l'enfant a pratiquement pris le contrôle de l'environnement familial par ses crises et ses comportements agressifs. Ces parents se posent continuellement les questions que nous venons d'énumérer.

Il s'agit, à n'en pas douter, d'un défi éducatif nouveau, entre autres par son ampleur. Pourtant, les milieux scolaires continuent de recourir à des interventions d'un autre âge qui n'ont que peu d'efficacité pour faire œuvre d'éducation auprès de ces jeunes. À titre d'exemples, la punition et la suspension scolaire, accompagnées plus ou moins subtilement d'une certaine culpabilisation des parents pour leurs lacunes familiales, continuent d'être des réactions usuelles face aux conduites agressives des élèves. 
Ces manières de faire perdurent malgré l'importance de la somme des connaissances que nous avons sur les pratiques exemplaires. Force est de constater que les milieux scolaires ont peu intégré ces savoirs. L'ensemble des recherches réalisées sur les programmes exemplaires indique pourtant clairement que les interventions les plus efficaces sont celles qu'on applique en amont, de manière préventive, et qui font appel à une étroite collaboration entre l'école et les parents.

L'ouvrage que proposent Maryse Paquin et Marie Drolet est important. Il témoigne de façon éloquente du vécu des parents d'enfants agressifs dans leurs relations avec les pairs à l'école. Plus spécifiquement, il s'agit d'une étude qui présente un certain nombre d'enfants ayant des comportements violents et leurs parents et qui dégage les défis et les enjeux de la collaboration entre les milieux scolaire et familial. Les chercheures tracent dans cet ouvrage un portrait saisissant de la réalité de la violence au préscolaire et au primaire. La recherche qu'elles présentent rend justice au fait que les familles ont changé, que les jeunes ont changé et que les écoles vont devoir modifier certaines de leurs pratiques pour mieux répondre aux défis posés par ces changements. La collaboration entre l'école et les parents de ces jeunes devient, à n'en pas douter, un des enjeux majeurs de ce changement de pratiques.

L'empowerment parental dans la collaboration entre les milieux scolaire et familial est la pierre d'assise de cet ouvrage; il s'exerce au moyen des gestes posés par les parents lorsqu'ils sont confrontés aux comportements violents de leur enfant à l'école. L'étude décrit d'ailleurs fort bien les différentes activités de collaboration de ces parents avec l'école. Le manque d'écoute et d'ouverture du personnel scolaire et le recours beaucoup trop fréquent à la suspension scolaire y sont particulièrement mis en relief. Pourtant, sans cette collaboration, il apparaît illusoire de pouvoir composer avec les comportements violents chez les enfants du préscolaire et du primaire, et encore plus de les prévenir. Nous savons tous comment les parents deviennent graduellement très réfractaires aux commentaires négatifs que contiennent presque toutes les communications envoyées par l'école au sujet de leur enfant. Ils en viennent à se retirer de ces échanges, à les ignorer ou à en faire carrément une occasion de conflit avec le personnel scolaire.

Tous ceux et celles qui, comme moi, travaillent en adaptation scolaire sont conscients que certains jeunes entrent à l'école «handicapés » par leurs comportements. Je suis heureux d'avoir la chance de vous présenter le travail de deux chercheures qui prennent vraiment à cœur le bien-être et la réussite éducative de ces jeunes. Elles soutiennent ici cette cause par une recherche originale, porteuse de changements, en décrivant précisément la 
situation de ces parents et de ces enfants, ainsi qu'en proposant des pistes d'action pour faire œuvre d'éducation auprès des ces familles. Je me fais le porte-parole des chercheurs et des praticiens œuvrant auprès d'eux pour les remercier pour la pertinence de cet ouvrage. 


\section{Note Au lecteur}

Choix du genre: Dans le présent ouvrage, le genre masculin est utilisé, sans discrimination, dans le seul but d'alléger le texte et d'en faciliter la lecture. 


\section{TABLE DES MATIÈRES}

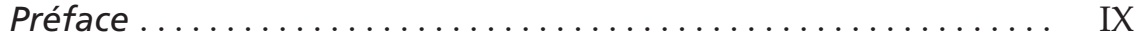

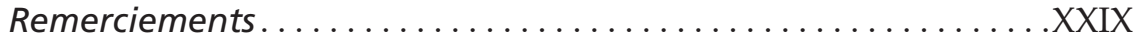

Introduction ............................ 1

Chapitre 1 L'état de la situation ................. 9

1. L'état de la violence au préscolaire et au primaire. . . . 11

1.1. L'intérêt accordé au phénomène........... 11

1.2. Les multiples définitions associées aux comportements violents . . . . . . . . . . . 12

1.3. Les types de comportements violents . . . . . . 15

1.4. Les caractéristiques individuelles, sociales et familiales. .................. 15

2. Le cadre légal face à la violence dans les écoles . . . . . . 19

2.1. La refonte des lois canadiennes touchant la jeunesse................... 20

2.2. La Politique de tolérance zéro du ministère ontarien de l'Éducation. . . . . . . . 21

2.3. Le code de conduite des écoles et leurs mesures disciplinaires. . . . . . . . . 21

2.4. Le Code de conduite provincial ............ 22

2.5. La Loi sur la sécurité dans les écoles de 1 'Ontario...................... 22

Chapitre 2 Les défis et les enjeux de la collaboration entre l'école et les parents............... 25

1. Le concept de collaboration............... 27

1.1. Les définitions de la collaboration et des concepts associés . . . . . . . . . . . . 27 
1.2. Les types de collaboration $\ldots \ldots \ldots \ldots \ldots \ldots, \quad 30$

1.3. Les conceptions de l'école au sujet de la collaboration avec les parents ......... 33

1.4. Le contexte propre au milieu scolaire franco-ontarien........................ 34

1.5. Le positionnement de la présente recherche.... 35

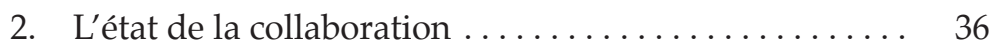

2.1. Pourquoi collaborer? .................. 36

2.2. Les comportements violents au préscolaire et au primaire...................... 38

2.3. Les diverses perspectives dans le temps ...... 39

2.4. Les nouvelles réalités familiales ........... 42

2.5. Les facteurs de soutien et les obstacles sous-jacents à la collaboration............ 44

2.6. Ce qu'en disent les études sur le milieu scolaire . $\quad 49$

3. Un court bilan des difficultés de collaboration....... 53

4. L'empowerment et les gestes posés par les parents ..... 55

4.1. L'empowerment: définitions............. 56

4.2. Les gestes d'empowerment posés par les parents .................... 58

\section{Chapitre 3 Le portrait des enfants ayant des comportements violents et celui} de leurs parents $\ldots \ldots \ldots \ldots \ldots \ldots \ldots \ldots \ldots, 61$

1. La méthodologie de recherche .............. 63

1.1. Les questions et les objectifs de la recherche.... 63

1.2. Les étapes de la recherche ............. 65

1.3. Les données sociodémographiques ........ 67

1.4. Les données sociodémographiques des enfants de 3 à 9 ans. . . . . . . . . . . . . . 71

1.5. Les limites de la recherche .............. 74

2. Les comportements violents des 3 à 6 ans......... 76

2.1. Le passage de la maison ou de la garderie

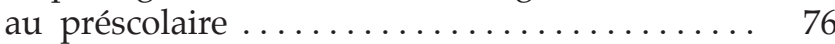

2.2. Les comportements violents .......... 77

2.3. La durée et la fréquence des comportements violents .......................... 82

2.4. Le lieu et le moment des comportements violents ......................... 83

2.5. La trajectoire des comportements violents ..... 84

2.6. Les pratiques éducatives parentales......... 85 
2.7. Les stresseurs familiaux ............ 87

2.8. Les facteurs explicatifs de la situation-problème à l'école . . . . . . . . . . . . . . . . . . . . . . 93

3. Les comportements violents des 6 à 9 ans......... 95

3.1. Le passage du préscolaire au primaire....... 96

3.2. Les comportements violents ........... 97

3.3. La durée et la fréquence des comportements violents ........................... 103

3.4. Le lieu et le moment des comportements violents ............................ 103

3.5. La trajectoire des comportements violents .... . 105

3.6. Les pratiques éducatives parentales . . . . . . . . . 107

3.7. Les stresseurs familiaux ................... 109

3.8. Les facteurs explicatifs de la situation-problème à l'école . . . . . . . . . . . . . . . . . . . . . . . 113

4. Le bilan du portrait de l'ensemble des participants de la recherche....................... 115

4.1. Les comportements violents des 3 à 9 ans ..... 115

4.2. La durée et la fréquence des comportements violents . . . . . . . . . . . . . . . . . . . . 120

4.3. Le lieu et le moment des comportements violents ........................ 121

4.4. La trajectoire des comportements violents ..... 122

4.5. Les pratiques éducatives parentales ayant un effet positif . .................... 123

4.6. Les pratiques éducatives parentales ayant un effet négatif...................... 124

4.7. Les stresseurs familiaux ............... 126

4.8. Les facteurs explicatifs de la situation-problème à l'école . . . . . . . . . . . . . . . . . . . . . . . . 127

Chapitre 4 La collaboration entre l'école et les parents. ... 131

1. Au sujet du concept de collaboration............. 133

1.1. L'implication parentale dans la vie scolaire de $l^{\prime}$ enfant. . . . . . . . . . . . . . . . . . . . . . . 134

1.2. L'implication parentale selon trois variables sociodémographiques................ 137

1.3. La communication entre les milieux scolaire et familial .......................... 142

1.4. Les difficultés de communication .......... 146

1.5. Un court bilan des résultats sur la collaboration .................... 150 
2. La participation des parents au processus de prise de décisions................... 151

2.1. L'invitation aux parents à participer à la recherche de solutions . . . . . . . . . . . . . . . 152

2.2. L'invitation aux parents à participer aux décisions ....................... 153

2.3. L'influence des parents sur les décisions déjà prises par l'école . . . . . . . . . . . . . . . . . 154

2.4. En résumé . . . . . . . . . . . . . . . . . . . . . . 157

3. La collaboration selon le profil des parents......... 158

3.1. Le profil des parents qui collaborent avec l'école ........................ 160

3.2. Le profil des parents qui ne collaborent pas avec l'école . . . . . . . . . . . . . . . . . . . . . 176

4. Les perceptions parentales à l'endroit du personnel scolaire ................................ 190

4.1. Des membres du personnel scolaire qui font leur marque.

4.2. Des membres du personnel scolaire qui alimentent la situation-problème . . . . . . . . . 195

4.3. D'autres perceptions parentales négatives à l'endroit du milieu scolaire. . . . . . . . . . . . . . . 200

4.4. En résumé . . . . . . . . . . . . . . . . . . . . . . . . . . . . 204

5. Les gestes d'empowerment posés par les parents ..... 205

5.1. Les gestes d'adaptation, d'opposition et de résistance.................... 205

5.2. L'adaptation pour une résolution de la situation-problème. . . . . . . . . . . . . . 206

5.3. L'opposition en vue d'une mobilisation des ressources. . . . . . . . . . . . . . . . . . . 208

5.4. La résistance comme autre option . . . . . . . . 211

5.5. En résumé . . . . . . . . . . . . . . . . . . . . . . . . 212

\section{Chapitre 5 La suspension scolaire pour comportements} violents ... . . . . . . . . . . . . . . . . . . . . . . . . 215

1. La problématique de la suspension scolaire . . . . . . . 217

1.1. L'origine de la mesure disciplinaire . . . . . . . . . 217

1.2. Des définitions ..................... 218

1.3. Les avantages et les inconvénients de la suspension scolaire . . . . . . . . . . . 218 
2. Les perceptions des parents face à la suspension scolaire externe ............................ 219

2.1. Les motifs du désaccord................ 220

2.2. L'ambivalence chez les plus ou moins en accord . . ......................... 227

2.3. Être d'accord tout en formulant certains souhaits ................... 229

2.4. Ne pas avoir d'opinion précise sur le sujet ..... 230

3. Une alternative à la suspension scolaire externe ..... 230

3.1. La suspension scolaire interne............ 231

3.2. Des mesures disciplinaires adaptées à chaque situation-problème $\ldots \ldots \ldots \ldots \ldots 232$

3.3. Les parents, des partenaires dans le processus de prise de décisions.................. 233

\section{Chapitre 6 Le bilan-synthèse des résultats} de la recherche .......................... 235

1. Le portrait des enfants . . . . . . . . . . . . . 237

2. Le portrait des parents ................. 238

3. La collaboration entre l'école et les parents ........ 240

4. La collaboration selon le milieu socioéconomique .... 244

5. Les facteurs de soutien sous-jacents à la collaboration . . . . . . . . . . . . . . . . . . . . . 246

6. Les obstacles sous-jacents à la collaboration........ 247

7. Les gestes d'empowerment posés par les parents ...... 250

8. Les perceptions des parents face à la suspension scolaire externe ........................ 253

9. Les perceptions des parents face aux autres mesures disciplinaires ..................... 254

10. Les éventuelles pistes d'intervention et de recherche..................... 256

Conclusion ........................................ 259

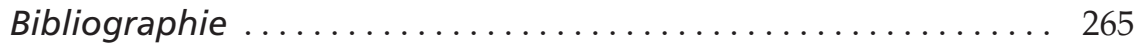




\section{Annexe 1 La grille d'entrevue parentale.}

Partie 1 - Cerner les perceptions des parents au sujet des comportements violents de leur enfant envers leurs pairs au préscolaire et au primaire.

Partie 2 - Décrire la problématique des parents d'enfants de 3 à 9 ans ayant des comportements violents envers leurs pairs au préscolaire et au primaire.

Partie 3 - Explorer les pratiques éducatives parentales ayant un effet positif et négatif sur les comportements violents de leur enfant . . . . . . . . . . . . . . . . . 293

Partie 4 - Tracer la trajectoire de la collaboration entre l'école et les parents, ainsi que les facteurs de soutien et les obstacles sous-jacents . . . . . . . . . . 294

Partie 5 - Cerner les perceptions des parents au sujet de l'influence, voire le contrôle, qu'ils exercent sur la situation-problème de leur enfant à l'école . . . . 295

Partie 6 - Dégager les occasions permettant aux parents de poser des gestes d'empowerment pour améliorer leur situation familiale et la situation-problème de leur enfant à l'école

Partie 7 - Cerner les perceptions des parents au sujet de la suspension scolaire et des autres mesures disciplinaires appliquées par l'école en réponse aux comportements violents de leur enfant envers ses pairs au préscolaire et au primaire. . . . . . . . . . . . . . . . . . . . . . . . 297

Partie 8-Données sociodémographiques ............ 298 
Annexe 2 Les catégories d'analyse .............. 303

A. L'ENFANT ............................. 305

- Caractéristiques personnelles................ 305

- Types de comportements violents............. 306

- Historique des comportements violents......... 306

- Raisons ou causes expliquant la présence des comportements violents selon le parent . . . . . 307

- Raisons ou causes expliquant la diminution des comportements violents selon le parent ...... 308

- Données sociodémographiques de l'enfant....... 308

B. LES PARENTS ET LA FAMILLE. . . . . . . . . . . 311

- Problématique des parents et de la situation familiale................. 311

- Compétences éducatives parentales ............ 312

- Empowerment parental .................... 313

- Données sociodémographiques des parents ...... 316

C. L'ÉCOLE................................. 319

- Information transmise aux parents ............. 319

- Attitudes, gestes et actions concrètes posés par l'école et par le personnel scolaire envers le parent .................... 319

- Attitudes, gestes et actions concrètes posés par le parent envers l'école et le personnel scolaire $\ldots \ldots \ldots \ldots \ldots \ldots \ldots \ldots$

- Perceptions des parents envers l'école . . . . . . . . . 321

- L'école perçue par les parents................ 321

- L'école perçue par l'enfant - Perceptions du code de conduite. .................. 322

Notices biographiques ............................ 325 



\section{$\begin{array}{lllllllll}T & A & B & L & E & A & U & X\end{array}$}

TABleAU 2.1 La typologie de l'implication parentale d'Epstein (1995) ...................... 31

TABLEAU 2.2 La typologie de l'investissement parental de Gordon (1996) . . . . . . . . . . . . . . . . . 32

TABleau 2.3 Les quatre modèles de l'influence entre l'école et les parents d'après Epstein (1992) ........... 41

TABLEAU 3.1 Les questions de recherche, principale et secondaires....................... 64

TABLEAU 3.2 Les sept objectifs de recherche ............... 64

TABLEAU 3.3 La répartition des parents selon le groupe d'âge $(n=60) \ldots \ldots \ldots \ldots \ldots \ldots \ldots \ldots \ldots \ldots \ldots \ldots, 68$

TABLEAU 3.4 Le statut des familles en fonction des tranches de revenu annuel $(n=60) \ldots \ldots \ldots \ldots \ldots \ldots . \ldots 9$

TABLEAU 3.5 L'occupation des parents $(n=60) \ldots \ldots \ldots \ldots \ldots \ldots$

TABLEAU 3.6 Le niveau de scolarité des parents $(n=60) \ldots \ldots \ldots .70$

TABLEAU 3.7 La langue parlée à la maison $(n=60) \ldots \ldots \ldots \ldots \ldots .71$

TABLEAU 3.8 La répartition des enfants selon le groupe d'âge

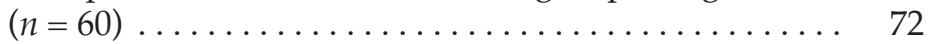

TABLEAU 3.9 Le statut des enfants dans la famille $(n=60) \ldots \ldots \ldots \quad 72$

TABleau 3.10 Le niveau scolaire des 3 à 9 ans $(n=60) \ldots \ldots \ldots \ldots$

TABLEAu 3.11 Les antécédents scolaires des 3 à 9 ans $(n=60) \ldots \ldots \quad 74$ 
TAbleau 3.12 Les antécédents médicaux des 3 à 9 ans $(n=60) \ldots . .75$

TABLEAU 3.13 Les comportements de violence physique des 3 à 6 ans $(n=23) \ldots \ldots \ldots \ldots \ldots \ldots \ldots \ldots \ldots \ldots \ldots$

TABLEAU 3.14 Les comportements de violence verbale avec ou sans gestes des 3 à 6 ans $(n=23) \ldots \ldots \ldots \ldots$

TABLEAU 3.15 Les problèmes internalisés des 3 à 6 ans $(n=23) \ldots \ldots 80$

TABLEAU 3.16 Les problèmes externalisés des 3 à 6 ans $(n=23) \ldots . .81$

TABLEAU 3.17 Les problèmes de socialisation des 3 à 6 ans $(n=23) \ldots \quad 82$

TABLEAU 3.18 La fréquence des comportements violents des 3 à 6 ans $(n=23) \ldots \ldots \ldots \ldots \ldots \ldots \ldots \ldots \ldots \ldots$

TABleaU 3.19 Le lieu et le moment des comportements violents des 3 à 6 ans $(n=23) \ldots \ldots \ldots \ldots \ldots \ldots \ldots \ldots \ldots \ldots$

TABLEAU 3.20 La trajectoire des comportements violents des 3 à 6 ans $(n=23) \ldots \ldots \ldots \ldots \ldots \ldots \ldots \ldots \ldots$

TABLEAU 3.21 Les pratiques éducatives parentales ayant un effet positif chez les 3 à 6 ans $(n=23) \ldots \ldots \ldots \ldots 86$

TABLEAU 3.22 Les pratiques éducatives parentales ayant un effet négatif chez les 3 à 6 ans $(n=23)$. . .

TABLEAU 3.23 Les stresseurs familiaux ayant un effet négatif sur les comportements des 3 à 6 ans $(n=23)$

TABLEAU 3.24 Les facteurs explicatifs de la situation-problème à l'école des 3 à 6 ans $(n=23) \ldots \ldots \ldots \ldots \ldots . \ldots 96$

TABleAu 3.25 Les comportements de violence physique

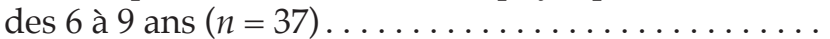

TABLEAU 3.26 Les comportements de violence verbale avec ou sans gestes des 6 à 9 ans $(n=37) \ldots \ldots \ldots .98$

TABLEAU 3.27 Les problèmes internalisés des 6 à 9 ans $(n=37) \ldots \ldots 100$

TABLEAU 3.28 Les problèmes externalisés des 6 à 9 ans $(n=37) \ldots \ldots 101$

TABLEAU 3.29 Les problèmes de socialisation des 6 à 9 ans $(n=37) \ldots 102$

TABLEAU 3.30 La fréquence des comportements violents des 6 à 9 ans $(n=37) \ldots \ldots \ldots \ldots \ldots \ldots \ldots \ldots \ldots$

TABleaU 3.31 Le lieu et le moment des comportements violents des 6 à 9 ans $(n=37) \ldots \ldots \ldots \ldots \ldots \ldots \ldots \ldots \ldots$ 
TABLEAU 3.32 La trajectoire des comportements violents des 6 à 9 ans $(n=37) \ldots \ldots \ldots \ldots \ldots \ldots \ldots \ldots \ldots$

TABLEAU 3.33 Les pratiques éducatives parentales ayant un effet positif chez les 6 à 9 ans $(n=37) \ldots \ldots \ldots \ldots .108$

TABLEAU 3.34 Les pratiques éducatives parentales ayant un effet négatif chez les 6 à 9 ans $(n=37) \ldots \ldots \ldots \ldots$

TABLEAU 3.35 Les stresseurs familiaux affectant négativement les comportements des 6 à 9 ans $(n=37) \ldots \ldots \ldots \ldots 112$

TABLEAU 3.36 Les facteurs explicatifs de la situation-problème à l'école chez les 6 à 9 ans $(n=37) \ldots \ldots \ldots \ldots \ldots . \ldots 114$

TABLEAU 3.37 Les comportements de violence physique

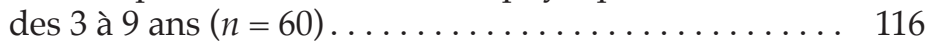

TABleaU 3.38 Les comportements de violence verbale avec ou sans gestes des 3 à 9 ans $(n=60) \ldots \ldots \ldots \ldots 117$

TABLEAU 3.39 Les problèmes internalisés des 3 à 9 ans $(n=60) \ldots \ldots 118$

TABLEAU 3.40 Les problèmes externalisés des 3 à 9 ans $(n=60) \ldots \ldots \quad 119$

TABLEAU 3.41 Les problèmes de socialisation des 3 à 9 ans $(n=60) \ldots 120$

TABLEAU 3.42 La fréquence des comportements violents des 3 à 9 ans $(n=60) \ldots \ldots \ldots \ldots \ldots \ldots \ldots \ldots \ldots \ldots \ldots \ldots$

TABleau 3.43 Le lieu et le moment des comportements violents

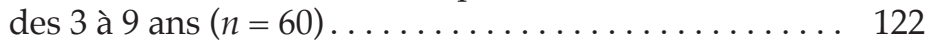

TABLEAU 3.44 La trajectoire des comportements violents chez les 3 à 9 ans $(n=60) \ldots \ldots \ldots \ldots \ldots \ldots \ldots \ldots$

TABLEAU 3.45 Les pratiques éducatives parentales ayant un effet positif chez les 3 à 9 ans $(n=60) \ldots \ldots \ldots \ldots 124$

TABLEAU 3.46 Les pratiques éducatives parentales ayant un effet négatif chez les 3 à 9 ans $(n=60) \ldots \ldots \ldots \ldots 125$

TABLEAU 3.47 Les stresseurs familiaux affectant négativement les comportements des 3 à 9 ans $(n=60) \ldots \ldots \ldots \ldots 127$

TABLEAU 3.48 Les facteurs explicatifs de la situation-problème à l'école chez les 3 à 9 ans $(n=60) \ldots \ldots \ldots \ldots \ldots 128$

TABleaU 4.1 Les types d'implication parentale dans la vie scolaire de l'enfant selon le milieu socioéconomique $(n=60) \ldots 137$

TABLEAU 4.2 L'implication parentale selon le nombre d'enfants par groupe d'âge $(n=43) \ldots \ldots \ldots \ldots \ldots \ldots \ldots$ 
TABLEAU 4.3 L'implication parentale selon le nombre de parents par niveau de scolarité $(n=43) \ldots \ldots \ldots \ldots \ldots \ldots . .140$

TABLEAU 4.4 L'implication parentale selon le nombre de familles par milieu socioéconomique $(n=43) \ldots \ldots \ldots \ldots \ldots, 141$

TABleAU 4.5 Les dimensions d'une bonne communication entre l'école et les parents selon le milieu socioéconomique $(n=60) \ldots \ldots \ldots \ldots \ldots \ldots \ldots, 145$

TABLEAU 4.6 Les dimensions d'une communication difficile entre l'école et les parents $(n=60) \ldots \ldots \ldots \ldots \ldots \ldots$

TABLEAU 4.7 L'invitation aux parents à participer à la recherche de solutions $(n=60) \ldots \ldots \ldots \ldots \ldots \ldots \ldots \ldots \ldots$

TABLEAU 4.8 L'invitation aux parents à participer aux décisions $(n=60) \ldots \ldots \ldots \ldots \ldots \ldots \ldots \ldots \ldots \ldots$

TABLEAU 4.9 L'influence des parents sur les décisions déjà prises par l'école selon le milieu socioéconomique $(n=60) \ldots 157$

TABLEAU 4.10 Le profil de collaboration des parents selon le milieu socioéconomique $(n=60)$

TABLEAU 4.11 Le profil des parents qui collaborent avec l'école $(n=25)$.

TABLEAU 4.12 Les dimensions de la reconnaissance de l'expertise de l'école $(n=25)$.

TABleau 4.13 Les dimensions de la reconnaissance de la complémentarité entre les milieux scolaire et familial $(n=23) \ldots \ldots \ldots \ldots \ldots \ldots \ldots, 166$

TABleAu 4.14 Les bienfaits de l'utilisation de services d'intervention sociale $(n=23) \ldots \ldots \ldots \ldots \ldots \ldots, 168$

TABleaU 4.15 Les constats des parents portant sur eux-mêmes $(n=22)$

TABLEAU 4.16 Les constats des parents portant sur la situation-problème de leur enfant $(n=11) \ldots \ldots \ldots 173$

TABleAu 4.17 Les dimensions d'un travail concerté entre tous les partenaires $(n=13)$

TABLEAU 4.18 Le profil des parents qui ne collaborent pas avec l'école $(n=19)$.

TABLEAU 4.19 Les dimensions de l'absence d'influence sur les décisions déjà prises par l'école $(n=19) \ldots \ldots . \quad 180$ 
TABleau 4.20 Les dimensions du sentiment d'impuissance des parents $(n=14) \ldots \ldots \ldots \ldots \ldots \ldots \ldots \ldots \ldots \ldots \ldots$

TABLEAU 4.21 La nature des jugements et les qualificatifs lourds de sens posés par l'école $(n=13) \ldots \ldots \ldots \ldots 184$

TABleaU 4.22 Les motifs de l'exclusion socioscolaire de l'enfant évoqués par l'école $(n=11) \ldots \ldots \ldots \ldots \ldots \ldots \ldots$

TABleAu 4.23 Les dimensions du désengagement de l'école à l'égard de l'enfant $(n=10) \ldots \ldots \ldots \ldots \ldots \ldots \ldots$

TABLEAU 4.24 Les dimensions du manque d'écoute et d'ouverture du personnel scolaire $(n=8) \ldots \ldots \ldots \ldots \ldots \ldots .187$

TABLEAU 4.25 Les dimensions d'une communication déficiente avec l'école $(n=8) \ldots \ldots \ldots \ldots \ldots \ldots \ldots \ldots \ldots$

TABLEAU 4.26 Les perceptions parentales à l'endroit du personnel

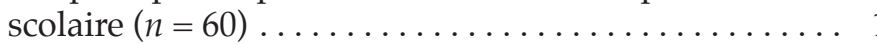

TABLEAU 4.27 Les perceptions parentales positives à l'endroit du personnel scolaire $(n=43)$

TABLEAU 4.28 La nature des perceptions parentales positives à l'endroit du personnel enseignant $(n=43) \ldots \ldots \ldots 193$

TABLEAU 4.29 La nature des perceptions parentales positives à l'endroit de la direction d'école $(n=43) \ldots \ldots \ldots \ldots . .194$

TABLEAU 4.30 Les perceptions parentales négatives à l'endroit du personnel scolaire $(n=31) \ldots \ldots \ldots \ldots$

TABLEAU 4.31 La nature des perceptions parentales négatives à l'endroit du personnel enseignant $(n=31) \ldots \ldots \ldots . \quad 197$

TABLEAU 4.32 La nature des perceptions parentales négatives à l'endroit de la direction d'école $(n=31) \ldots \ldots \ldots \ldots . .199$

TABLEAU 4.33 D'autres perceptions parentales négatives à $l^{\prime}$ endroit du milieu scolaire $(n=31) \ldots \ldots \ldots \ldots 200$

TABLEAU 4.34 La nature des perceptions parentales négatives à l'endroit du personnel scolaire en général $(n=31) \ldots 202$

TABLEAU 4.35 La nature des perceptions parentales négatives à l'endroit de l'école en général $(n=31) \ldots \ldots \ldots \ldots 203$

TABleau 4.36 Les gestes d'adaptation posés par les parents

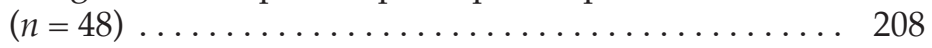

TABLEAU 4.37 Les gestes d'opposition posés par les parents $(n=48)$ 
XXVIII La violence au préscolaire et au primaire

TABLEAU 4.38 Les gestes de résistance posés par les parents

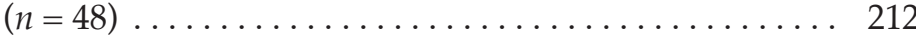

TABLEAU 5.1 Les perceptions des parents face à la suspension scolaire externe $(n=60) \ldots \ldots \ldots \ldots \ldots \ldots \ldots$

TABleau 5.2 Les motifs des parents en désaccord avec la suspension scolaire externe $(n=39) \ldots \ldots \ldots 225$

TABleaU 5.3 Les motifs des parents plus ou moins en accord avec la suspension scolaire externe $(n=11) \ldots \ldots \ldots 228$

TABLEAU 5.4 Les motifs des parents en accord avec la suspension scolaire externe $(n=6) \ldots \ldots \ldots \ldots \ldots \ldots \ldots$

TABLEAU 5.5 Les motifs des parents qui n'ont pas d'opinion précise sur la suspension scolaire externe $(n=4) \ldots \ldots \ldots 230$

TABLEAU 5.6 Les perceptions des parents face à la suspension scolaire interne $(n=60) \ldots \ldots \ldots \ldots \ldots \ldots \ldots \ldots \ldots \ldots \ldots$

TABLEAU 5.7 Les perceptions des parents face aux autres mesures disciplinaires $(n=60) \ldots \ldots \ldots \ldots \ldots \ldots \ldots \ldots \ldots \ldots \ldots \ldots$

TABLEAU 6.1 Le bilan-synthèse du portrait des enfants $(n=60) \ldots . .238$

TABLEAU 6.2 Le bilan-synthèse du portrait des parents $(n=60) \ldots . .240$

TABLEAU 6.3 Le bilan-synthèse de la collaboration entre l'école et les parents $(n=60) \ldots \ldots \ldots \ldots \ldots \ldots \ldots \ldots$

TABLEAU 6.4 Le bilan-synthèse de la collaboration selon le milieu socioéconomique $(n=60) \ldots \ldots \ldots \ldots \ldots \ldots \ldots \ldots$

TABLEAU 6.5 Le bilan-synthèse des facteurs de soutien sous-jacents à la collaboration $(n=25) \ldots \ldots \ldots \ldots \ldots \ldots \ldots \ldots$

TABLEAU 6.6 Le bilan-synthèse des obstacles sous-jacents à la collaboration $(n=19) \ldots \ldots \ldots \ldots \ldots \ldots \ldots$

TABLEAU 6.7 Le bilan-synthèse des gestes d'empowerment posés par les parents $(n=48) \ldots \ldots \ldots \ldots \ldots \ldots 252$

TABLEAU 6.8 Le bilan-synthèse des perceptions des parents face à la suspension scolaire externe $(n=60) \ldots \ldots \ldots 254$

TABLEAU 6.9 Le bilan-synthèse des perceptions des parents face aux autres mesures disciplinaires $(n=60) \ldots \ldots .256$ 


\section{REMERCIEMENTS}

Maryse Paquin et Marie Drolet 

Le présent ouvrage est le fruit de quatre années de recherche. Les résultats de l'étude initiale ${ }^{1}$ ont été remis au Centre national de prévention du crime du ministère de la Justice du Canada, dans le cadre du Programme de partenariat en prévention du crime. Nous tenons à les remercier pour leur appui financier de même que le Service de la recherche de l'Université d'Ottawa, dans le cadre du Programme de partenariat en sciences humaines et du Programme d'initiatives de recherche collaboratives interfacultaires. Sans ce financement ainsi que la subvention du Programme d'aide à l'édition savante de la Fédération canadienne des sciences humaines, obtenus au terme du processus d'arbitrage, ont permis la réalisation avec succès de toutes les étapes de cette recherche.

De plus, nous désirons exprimer notre profonde gratitude aux parents qui ont participé à cette recherche. Sans leur collaboration et leur disponibilité, ainsi que l'ouverture dont ils ont fait preuve, il aurait été impossible d'atteindre les objectifs que nous nous sommes fixés. Partager le vécu de ces familles, parfois heureux, parfois malheureux, et ce, dans le plus grand respect, a été une préoccupation constante.

Nous adressons aussi nos remerciements à madame Louise Lafortune pour son soutien et à madame Pauline Provencher, pour l'avoir assistée dans le processus d'arbitrage de la collection Éducation-Recherche, de même qu'aux Presses de l'Université du Québec pour le dévouement témoigné tout au long de ce projet d'édition.

Nous désirons également souligner la participation d'une collaboratrice, Rachel Hasan et de nombreuses assistantes de recherche, dont Nadya Chartier, ayant contribué au succès de cette entreprise. D'autres organismes de la région d'Ottawa y ont également participé de près ou de loin: le Conseil des écoles catholiques de langue française du CentreEst de l'Ontario, le Conseil des écoles publiques de l'Est de l'Ontario, ainsi que leurs directions d'écoles, le Centre psychosocial pour enfants et familles, le Département des techniques de services correctionnels de la Cité Collégiale, le Centre de ressources de la Basse-ville, le Mouvement d'implication francophone d'Orléans et le Réseau des services de garde

1. Des extraits de cet ouvrage sont tirés de Marie Drolet et Maryse Paquin en collaboration avec Magnolia Soutyrine, Rachel Hasan et Caroline Ringuet (2003). Pour contrer la violence chez les enfants de 3 à 9 ans : une collaboration entre les écoles et parents concernés, occasions d'empowerment pour les parents. Rapport technique, texte inédit remis au Centre national de prévention du crime, Justice Canada, juillet, 205 p. 
francophone de l'Ontario. Sans leur implication et leur engagement face à la question de la réussite scolaire dans le champ de l'enfance en difficulté, ce projet n'aurait pu être mené à bien.

En terminant, nous remercions sincèrement le professeur Égide Royer, de l'Université Laval, qui a gentiment accepté de signer la préface. Nous formulons le souhait que les pistes éventuelles pour l'intervention et la recherche qui sont contenues dans le présent ouvrage puissent s'avérer utiles et inspirantes. Donner suite à ces pistes, tout comme aux propos des parents constamment en quête de solutions, ne pourra selon nous, que contribuer au mieux-être des enfants, de leur famille, de l'école, et de tous ceux et celles qui sont impliqués de près ou de loin dans un processus de résolution de situations-problèmes, au bénéfice de toutes les parties. 
Introduction 

Depuis une dizaine d'années, on porte une attention particulière à la violence en milieu scolaire, soit aux enfants ayant des comportements violents envers leurs pairs (Astor, 1995; Herrenkohl, Huang, Kosterman, Hawkins, Catalano et Smith, 2001; Saravanamuttoo, 2001). À ce sujet, Tremblay (2000) précise que c'est entre l'âge de 2 et 3 ans que les enfants sont les plus violents physiquement. Les comportements violents, surtout lorsqu'ils sont fréquents et stables, deviennent un important facteur prédicteur de délinquance à l'adolescence ou même de criminalité à l'âge adulte (Farrington, 2000; Kosterman, Graham, Hawkins, Catalano et Herrenkohl, 2001 ; Leblanc, 1999 ; Stormont, 2000 ; Tremblay, 1995). En ciblant les enfants de 3 à 9 ans, le but ultime visé par la prévention et l'intervention précoces est d'éviter l'enchaînement de problèmes plus graves (Eisenberg, Guthrie, Murphy et Shepard, 1999; Tremblay, 2000).

C'est dans la foulée de ces constats et des résultats obtenus dans le cadre d'une étude exploratoire, qui mettait en relief l'augmentation de signalements de comportements violents chez les enfants du préscolaire et du primaire, que nous avons décidé de poursuivre nos recherches. Nos travaux se fondent sur les plus récents écrits en matière de prévention et d'intervention précoces, en ayant comme prémisse que les parents s'avèrent des partenaires-clés pour aider l'école à contrer les comportements violents chez leur enfant. Un tel constat a également reçu l'appui de la Fédération nationale des conseillers scolaires francophones (2003). Toutefois, selon bon nombre d'auteurs, l'enjeu stratégique pour y arriver semble reposer sur une bonne collaboration entre les milieux scolaire et familial, dans une démarche d'empowerment parental.

Dans ce contexte, le présent ouvrage s'intéresse non seulement aux enfants de 3 à 9 ans qui fréquentent les écoles élémentaires de langue française de la région d'Ottawa, mais également et surtout à leurs parents. Les conseils scolaires et de nombreux collaborateurs œuvrant auprès de cette clientèle ont été mis à contribution afin de favoriser leur participation à la présente recherche. À cet effet, ce projet a reçu l'appui de la Direction des services en langue française du ministère de l'Éducation et du ministère des Services sociaux et communautaires de l'Ontario. Fort de ces appuis, un travail de partenariat s'est installé dans l'optique d'une synergie entre les domaines éducationnel, social et communautaire. C'est dans le prolongement de cette synergie qu'ont été formulées les questions de recherche auxquelles se sont intéressées les auteures, soit la question principale: Quels sont les défis et les enjeux de la collaboration entre l'école et les parents d'enfants de 3 à 9 ans afin de contrer leurs comportements violents envers leurs pairs au préscolaire et au primaire? Puis, des questions de recherche secondaires ont été formulées, soit: Quel est le portrait des enfants de 3 à 9 ans ayant des comportements violents envers leurs pairs à l'école 
et de leurs parents? Quels sont les éléments constituant un contexte de collaboration et de non-collaboration entre l'école et les parents? Quels sont les facteurs de soutien et les obstacles sous-jacents? Quels sont les gestes d'empowerment posés par les parents leur permettant d'exercer une influence, voire un contrôle, sur la situation-problème de leur enfant à l'école? Finalement, quelles sont les mesures disciplinaires appliquées par l'école permettant de contrer les comportements violents des 3 à 9 ans et de ne pas entraver leur processus de scolarisation et de socialisation? Afin d'apporter des pistes de réponses à ces questions, nous avons formulé des objectifs de recherche. Leur atteinte permet d'exposer les résultats obtenus tout au long des chapitres du présent ouvrage.

Dans un premier temps, nous présentons la problématique des enfants de 3 à 9 ans ayant des comportements violents envers leurs pairs au préscolaire et au primaire. Au départ, nous exposons les facteurs de risque. Ceux-ci permettent de mieux situer la complexité de cette problématique. Puis, nous présentons le cadre légal relatif au contrôle de la violence dans les écoles, notamment celui qui est propre au milieu scolaire de l'Ontario. Dans cette province, intervenir contre la violence signifiait déjà depuis une décennie d'appliquer la Politique de tolérance zéro (Ministère de l'Éducation de l'Ontario, 1994). Ainsi, le document intitulé: Pour des écoles sans violence: une politique précise les enjeux liés au contrôle de la violence en milieu scolaire dans cette province. Avec l'adoption de cette Politique, les écoles avaient dorénavant la responsabilité de mettre en œuvre un code de conduite qui déterminait les conséquences qu'elles appliqueraient en réponse aux comportements violents des élèves. Une de ces mesures disciplinaires consistait en la suspension scolaire, et ce, dès le début de la scolarisation de l'enfant. À la suite de l'adoption, en 2000, de la Loi sur la sécurité dans les écoles (Ministère de l'Éducation de l'Ontario, 2000) et du Code de conduite provincial (Ministère de l'Éducation de l'Ontario, 2001a), le durcissement du climat provoqué par l'application de mesures coercitives uniformes était propice à générer un contexte davantage conflictuel entre l'école et les parents. Un tel contexte pouvait affecter les garderies scolaires qui devaient appliquer des mesures concomitantes à celles de l'école. Il pouvait également toucher les services sociaux à qui sont référés les enfants ayant des comportements violents et leurs parents afin qu'ils puissent recevoir de l'aide. C'est pourquoi, afin de tracer le profil des parents qui collaborent et de ceux qui ne collaborent pas avec l'école, il importait de mieux comprendre les facteurs de soutien et les obstacles sous-jacents à la collaboration entre l'école et les parents. C'est pour y parvenir que nous avons mené la présente recherche, qui consistait à réaliser des entrevues auprès de soixante parents d'enfants de 3 à 9 ans ayant des comportements violents envers leurs pairs au préscolaire et au primaire. Pour faire partie de l'échantillon, l'enfant devait faire l'objet d'une suspension ou d'une 
possibilité de suspension scolaire en réponse à de tels comportements. L'échantillon est constitué de parents volontaires ayant accepté de réaliser une entrevue téléphonique. La moitié d'entre eux ont réalisé une entrevue structurée et l'autre, une entrevue semi-structurée. La durée des entrevues varie de trente minutes à deux heures, en fonction de la disponibilité que les parents ont accepté de consentir.

Nous enchaînons ensuite avec le concept de collaboration entre l'école et les parents. Nous accordons une attention toute particulière à ce concept puisqu'il est indissociable de la problématique de la violence chez les enfants de 3 à 9 ans. Si les parents sont moins présents dans la vie scolaire de leur adolescent, il en va tout autrement lorsque leur enfant en bas âge fait face à une situation-problème à l'école. Toutefois, d'entrée de jeu, nous établissons une distinction entre les concepts d'implication et de participation parentales, souvent associés à celui de collaboration. Par la suite, les facteurs de soutien et les obstacles sous-jacents à la collaboration entre l'école et les parents sont circonscrits. Puis, nous nous intéressons à un autre concept-clé, celui de l'empowerment parental. Ce concept retient l'attention puisqu'il explique les réactions parentales face à leur collaboration ou leur non-collaboration avec l'école. Dans certains cas, les parents poseront des gestes d'adaptation, d'opposition et de résistance afin d'exercer une plus grande influence, voire un contrôle, sur la situation-problème de leur enfant à l'école.

Nous brossons ensuite le portrait des enfants violents de 3 à 9 ans et de leurs parents. Ce portrait permet de mieux saisir toute la situation vécue par les familles de l'échantillon. Nous décrivons également la méthodologie de recherche. Celle-ci a donné lieu à une analyse qualitative des données, présentée d'abord selon le regroupement des enfants de 3 à 6 ans et de leurs parents, puis selon celui des enfants de 6 à 9 ans et de leurs parents. Ces regroupements tiennent compte des deux importantes transitions qui surviennent dans la vie de l'enfant au cours de cette période, soit celle entre la maison/garderie et le préscolaire, et celle entre le préscolaire et le primaire. Ces regroupements se situent également dans le cadre d'action des professionnels qui œuvrent dans le domaine de la petite enfance, soit auprès d'enfants du préscolaire, ou auprès d'enfants qui sont au début de leur scolarisation, soit au primaire. L'analyse des données fait notamment ressortir les perceptions des parents face aux comportements violents de leur enfant, leurs pratiques éducatives, les stresseurs familiaux qu'ils affrontent, ainsi que les facteurs explicatifs de la situation-problème de leur enfant à l'école. Cette analyse des données contribue à enrichir la connaissance des perceptions qu'entretiennent les parents de la présente recherche face à leur milieu de vie familial et face au contexte scolaire dans 
lequel évolue leur enfant. Tout au long de ce chapitre et des suivants, nous présentons des tableaux contenant le nombre et le pourcentage des répondants, afin de faciliter la lecture de l'analyse qualitative des données.

L'analyse des données porte ensuite sur les perceptions qu'entretiennent les parents en regard de trois concepts sous-jacents à la collaboration, soit leur implication dans la vie scolaire, la communication entre les milieux scolaire et familial ainsi que la participation des parents au processus de prise de décisions concernant leur enfant à l'école. En somme, ces trois concepts se révèlent des indices précieux quant à la nature de la collaboration ou de la non-collaboration qui s'établit entre l'école et les parents. Nous cernons des facteurs de soutien et des obstacles ainsi que les gestes d'empowerment posés par les parents. Certains résultats sont présentés en fonction du milieu socioéconomique des familles, de l'âge de l'enfant et du niveau de scolarité des parents, car ces variables sociodémographiques déterminent différentes manières de collaborer avec l'école.

Nous nous sommes également intéressées à la suspension scolaire, puisqu'elle représente un enjeu crucial lié à la problématique des enfants de 3 à 9 ans ayant des comportements violents envers leurs pairs au préscolaire et au primaire. C'est pourquoi nous ferons le point sur les perceptions que les parents entretiennent face à l'application de cette mesure disciplinaire en réponse à de tels comportements. Après avoir exposé les principaux constats, nous présentons une alternative à la suspension scolaire externe, soit la suspension interne, de même que des suggestions d'application de mesures disciplinaires adaptées à chaque situation-problème. Toutes ces suggestions sont formulées dans l'optique que les parents soient des collaborateurs, voire des partenaires à part entière, dans le processus de prise de décisions concernant leur enfant à l'école.

Finalement, nous dressons le bilan-synthèse des résultats de l'ensemble de la recherche. Nous résumons les caractéristiques des enfants et de leurs parents. Par la suite s'enchaînent les principaux constats qui se dégagent de l'analyse des données quant à la collaboration entre l'école et les parents, aux facteurs de soutien et obstacles sous-jacents à cette collaboration, aux gestes d'empowerment posés par les parents, ainsi qu'à leurs perceptions de la suspension scolaire comme mesure disciplinaire appliquée en réponse aux comportements violents de leur enfant envers leurs pairs au préscolaire et au primaire. Pour terminer, nous présentons des pistes éventuelles d'intervention et de recherche dans le domaine de la prévention et de l'intervention précoces. Tout en témoignant des 
besoins exprimés par les familles ayant participé à notre recherche, ces pistes mènent à de nouveaux questionnements et invitent à poursuivre la réflexion et l'action déjà amorcées.

Compte tenu de l'importance de la problématique de la violence au préscolaire et au primaire et de ses conséquences à long terme, nous concluons à la nécessité de solutionner tôt les situations-problèmes. Pour ce faire, toutes les instances doivent être interpellées, soit d'abord et avant tout les parents, les enfants, l'école, les services sociaux et le milieu communautaire. Pour nous, sans véritable collaboration, voire partenariat, entre ces instances, toute tentative de contrer les comportements violents des enfants de 3 à 9 ans est vouées à l'échec. Toutefois, nous prévenons le lecteur que le fait de donner la parole aux parents suscite énormément de réactions. C'est que leur lecture de la situation-problème peut parfois apparaître inconfortable et dérangeante, notamment aux yeux du personnel scolaire. C'est pourquoi cette problématique commande de poursuivre non seulement la recherche, mais également la prévention et l'intervention précoces dans le champ de la violence au préscolaire et au primaire, en visant en bout de ligne à assurer le mieux-être des enfants, de leur famille, de l'école, de la communauté, voire de toute une société. 



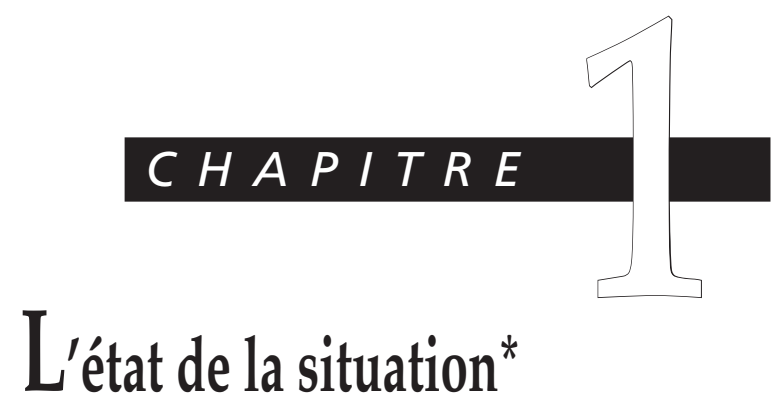

* Des extraits de ce chapitre sont tirés de: M. Paquin (2004). «Liminaire: la violence en milieu scolaire: une problématique qui concerne la famille, l'école et la communauté, voire la société», Éducation et francophonie, numéro spécial sur la violence à l'école, 32(1), p. 1-14. 

Le premier chapitre de cet ouvrage présente le contexte à partir duquel nous abordons le phénomène de la violence chez les enfants de 3 à 9 ans. Il contient deux parties dont la première porte sur l'état de la violence au préscolaire et au primaire, les multiples définitions, les types et les facteurs de risque associés aux comportements violents chez les enfants. Quant à la seconde partie, elle traite du cadre légal canadien et ontarien face à la violence dans les écoles.

\section{L'ÉTAT DE LA VIOLENCE AU PRÉSCOLAIRE ET AU PRIMAIRE}

La première partie vise plus particulièrement à dresser l'état de la violence au préscolaire et au primaire en tentant de répondre aux questions suivantes: Les comportements violents chez les enfants de 3 à 9 ans sontils plus fréquents qu'avant? S'entend-on sur une définition commune des comportements violents? De quels types de comportements violents s'agit-il? Quels sont les facteurs de risque associés à l'émergence de tels comportements chez ces enfants, soit leurs caractéristiques individuelles, sociales et familiales? Nous proposons plusieurs pistes de réponses en fonction des plus récents écrits portant sur ces questions.

\subsection{L'INTÉRÊT ACCORDÉ AU PHÉNOMÈNE}

Depuis quelques années, la question est sur toutes les lèvres: Assistonsnous à une flambée de la violence au préscolaire et au primaire? Le débat demeure toujours ouvert et les avis s'avèrent des plus partagés. Pour certains, les comportements violents chez les enfants sont nettement plus fréquents qu'auparavant (Bullis, Walker et Sprague, 2001). De plus, ils continueraient même de croître (Danyluk, 1995) en indiquant davantage d'hostilité et de cruauté envers les pairs (Kinnear, 1995). Pour d'autres, qui défendent le point de vue inverse, le manque de données objectives ne permettrait pas de circonscrire l'ampleur du phénomène (Funk, 2001; Hébert, 2001).

Au sujet de la violence à l'école, Debarbieux (2001) rappelle que le nombre de méfaits commis par les élèves demeure proportionnellement restreint par rapport à l'ensemble de la clientèle scolaire. Par ailleurs, l'intérêt médiatique accru des dernières années sur la question des homicides à multiples victimes commis par des adolescents (Lemonick, 2002), avive les inquiétudes et contribue à créer une fausse image du phénomène (Charlot et Émin, 2001; Martichoux, 2000 ; Trépanier, 1999). Un consensus émerge 
à l'effet que la majorité de la population perçoit maintenant cette violence comme un enjeu crucial (Carrington, 1999; Lorrain, 1999; Verlinden, Hersen et Thomas, 2000). Par conséquent, l'importance accordée au contrôle de la violence dans les écoles, et ce, dès le plus jeune âge, semble augmenter d'année en année (Tremblay, Boulerice, Harden, McDuff, Pérusse, Pihl et Zoccolillo, 1996; Vitaro et Gagnon, 2000). Plusieurs auteurs cherchent à saisir la problématique tant des agresseurs que des victimes (Conseil national de prévention du crime, 2002; Fortin, 2002 ; Kosterman, Graham, Hawkins, Catalano et Herrenkohl, 2001). D'autres arrivent à la conclusion que les manifestations de la violence dans les jeux vidéo, les films et les émissions de télévision contribuent de manière significative à sa banalisation (Freedman, 2002; Huesmann, Moise-Titus, Podolski et Eron, 2003). Pour ces raisons, un intérêt se fait sentir sur la question de la prévention et l'intervention précoces des comportements violents (Loeber et Farrington, 2000). C'est pourquoi, depuis un certain temps, une attention particulière est portée aux jeunes enfants (Herrenkohl, Huang, Kosterman, Hawkins, Catalano et Smith, 2001; Saravanamutoo, 2001) puisque les premières manifestations de violence apparaissent en très bas âge. $D^{\prime}$ une part, le point culminant de la violence physique dans le développement social des enfants apparaît, selon Tremblay (2000), entre l'âge de 2 et 3 ans. D'autre part, lorsqu'ils se maintiennent au préscolaire et au primaire et, surtout, lorsqu'ils sont fréquents et stables, les comportements violents deviennent un indicateur important de violence ou même de délinquance à l'adolescence ou à l'âge adulte (Farrington, 2000 ; Kosterman, Graham, Hawkins, Catalano et Herrenkohl, 2001; Leblanc, 1999; Stormont, 2000; Tremblay, 1995). En ciblant les 3 à 6 ans et le cycle préscolaire, de même que les 6 à 9 ans et le cycle primaire, le but ultime visé est de diminuer les risques de délinquance et de criminalité futures (Eisenberg, Guthrie, Murphy et Shepard, 1999; Tremblay, 2000).

\subsection{LES MULTIPLES DÉFINITIONS ASSOCIÉES AUX COMPORTEMENTS VIOLENTS}

Tout d'abord, qu'entend-on par " comportements violents » ? Cette définition peut prendre des formes multiples selon les perspectives adoptées par les chercheurs. Selon Duhamel-Maples (1996, p. 41), «une multitude d'appellations sont généralement utilisées pour désigner les comportements violents chez l'enfant». De plus, les définitions sont répertoriées dans au moins six champs professionnels. Si les divers termes sont parfois utilisés comme s'il s'agissait de concepts interchangeables, ils renvoient toutefois à des réalités fort différentes, d'après la description qu'en donnent les ouvrages consultés. 
En pédopsychiatrie, selon la $4^{\mathrm{e}}$ édition du Diagnostic and Statistical Manual of Mental Disorders, communément appelé le DSM-IV (American Psychiatric Association, 1996, p. 63), il est notamment question du Trouble du déficit de l'attention avec hyperactivité (TDAH) et impulsivité (TDAHI). Les trois composantes de ce trouble se rapportent à la difficulté chez l'enfant de se concentrer, jumelée à un haut niveau d'activité et à un manque de contrôle de soi. Dans le DSM-IV (American Psychiatric Association, 1996, p. 68), il est également question du Trouble oppositionnel avec provocation (TOP), qui comprend un ensemble de comportements négativistes, hostiles et provocateurs: colère, contestation, opposition, susceptibilité, attitude blâmante, méchanceté, etc. Le DSM-IV (American Psychiatric Association, 1996, p. 66) identifie aussi le Trouble des conduites (TC), qui semble même être de loin le plus grave problème pouvant affecter la vie de l'enfant (Gagnon et Vitaro, 2000). Ce trouble réfère à un ensemble de conduites, répétitives et persistantes, dans lequel sont bafoués les droits fondamentaux d'autrui ou les normes et règles sociales, telles que la cruauté physique envers les personnes ou les animaux, la destruction de biens matériels, la violation grave de règles établies, etc. Finalement, un quatrième Trouble recensé (American Psychiatric Association, 1996, p. 69) concerne le comportement perturbateur (TCP). Ce dernier se situe à mi-chemin entre le Trouble oppositionnel avec provocation et le Trouble des conduites. Sur la base d'une approche clinique, l'ensemble de ces Troubles «sont définis à partir de symptômes ayant persisté pendant au moins six mois, à un degré qui est inadapté et ne correspond pas au niveau de développement de l'enfant» (American Psychiatric Association, 1996, p. 63).

En psychologie, plusieurs termes ont été répertoriés, soit les désordres, les handicaps ou les perturbations d'ordre émotionnel (Kauffman, 1997). Ceux-ci renvoient à des conditions de souffrance morale chez l'enfant qui sont soit ponctuelles, soit persistantes. Elles comprennent l'anxiété, la maniacodépression, l'excessivité / compulsivité, etc. Toutefois, c'est le terme agressivité qui semble le plus couramment utilisé pour décrire l'état psychologique de l'enfant (Christenson, Hirsch et Hurley, 1997). Les plus récentes taxonomies distinguent même les comportements agressifs selon leur caractère internalisé ou externalisé (Gagnon et Vitaro, 2000). Notamment, l'agressivité externalisée se subdivise en deux types, soit les comportements réactifs (avec provocation) et proactifs (sans provocation).

Dans le domaine de l'éducation, il est plutôt question de problèmes, de difficultés ou de troubles de comportement (Fortin, Toupin, Pauzé, Déry et Mercier, 1996). Ceux-ci renvoient à l'incapacité chez l'enfant de fonctionner de manière «normale» dans le cadre des règles fixées par l'institution scolaire. Il est également question d'indiscipline (Ministère de l'Éducation du Québec, 1992), un terme que le personnel enseignant 
associe généralement à la gestion de classe. Jeffrey et Simard (2000, p. 17) définissent la gestion de classe comme étant « un travail qui touche notamment aux règles de la classe et aux conséquences à leur manquement, de même qu' aux routines de fonctionnement et à leur ordonnancement ». Selon ces mêmes auteurs, les premières semaines de l'année scolaire se révèlent particulièrement critiques, puisque $c^{\prime}$ est au cours de cette période que les enseignants tentent $\mathrm{d}$ 'anticiper et de prévenir les problèmes liés à cette gestion. À cet effet, ils consacrent beaucoup de temps à communiquer les règles, les procédures et leurs attentes face au comportement des élèves. C'est également au cours de cette période qu'ils cherchent à se faire une idée sur chacun d'eux.

Quant au domaine de la psychopédagogie, les plus récentes études retiennent le concept d'enfants à risque (Paquin et Drolet, 2000). Ce concept s'intéresse plus particulièrement à la possibilité qu'un enfant devienne tour à tour un agresseur ou une victime de la violence. À ce concept sont associés ceux des facteurs de protection et de résilience. Selon Fortin, Toupin, Pauzé, Déry et Mercier (1996), qui étudient la question sous des angles multifactoriels, les comportements violents sont le résultat de l'accumulation d'un certain nombre de facteurs de risque qui augmentent la probabilité de voir apparaître la situation-problème chez l'enfant. Par ailleurs, la présence d'un certain nombre de facteurs de protection diminue la probabilité de la voir émerger. Quant aux facteurs de résilience, ils correspondent à la capacité de l'enfant de faire face à des conditions adverses avec succès.

Finalement, dans le domaine de la criminologie, en contrevenant aux règles, à l'ordre et aux lois en vigueur, l'enfant posant des gestes violents est considéré comme étant un futur délinquant (Leblanc, 1999), ainsi qu'un criminel en devenir (Carrington, 1999).

En ce qui a trait au domaine du service social, ce champ porte une attention particulière au contexte dans lequel émerge le comportement violent (Hasan, Drolet et Paquin, 2003) qui peut être abordé davantage sous l'angle de l'antisociabilité (Patterson, 1992) et des comportements inadaptés socialement (Bishop et Rothbaum, 1992). Ces comportements renvoient à la difficulté d'établir des relations sociales satisfaisantes, de manière individuelle ou collective. Le peu d'habiletés ou de compétences chez l'enfant permettant de nouer des liens solides peut parfois révéler un manque de sociabilité (Pearl, 1987; Tarabulsy et Tessier, 1996).

Tous ces termes illustrent bien la diversité conceptuelle qui règne tant entre les différents domaines qu'au sein d'un même champ professionnel. D'où l'importance de préciser la définition de «comportements violents» qui est privilégiée dans le présent ouvrage. 
Selon les concepteurs de la Conflict Tactics Scale, Straus et Gelles (1990, p. 65), avoir des comportements violents signifie «agir avec l'intention réelle ou perçue de blesser une autre personne physiquement, matériellement ou symboliquement». Comme la notion d'intention occupe une place prépondérante dans cette définition et qu'elle ne tient pas assez compte du résultat de cette intention, Bouchard et Tessier (1996, p. 55) proposent la variante suivante, soit «toute action qui, dans une situation de conflit, compromet ou risque de compromettre l'intégrité ou le bien-être psychologique et physique de la personne». C'est la seconde définition qui a été privilégiée aux fins du présent ouvrage parce qu'elle rejoint davantage l'idée que l'enfant de 3 à 9 ans n'est pas toujours conscient des conséquences de l'acte posé, qu'il soit prémédité ou non. De même, ses comportements violents peuvent être de plusieurs types.

\subsection{LES TYPES DE COMPORTEMENTS VIOLENTS}

Selon les auteurs, les comportements violents peuvent consister en une atteinte physique (Bouchard, Pelchat et Boudreault, 1996a; Centre national d'information sur la violence dans la famille, 1997; Hoff, 1994), comme par exemple: mordre, frapper, tirer les cheveux, bousculer, donner des coups de pied ou des coups de poing. Ils peuvent également être de nature verbale et s'accompagner de gestes (Bouchard, Pelchat et Boudreault, 1996a; Ohsako, 1997), comme par exemple: détruire ses choses ou celles des autres, lancer, casser ou donner des coups de pied sur des objets ou cracher. Finalement, ils peuvent être de nature verbale sans s'accompagner de gestes (Anatrella, 1995), comme par exemple: insulter, blâmer, intimider, mentir, menacer de frapper ou de lancer des choses.

Quel que soit le type de comportements violents, ceux qui posent davantage de problèmes se présentent de manière fréquente et stable dans divers milieux et à travers le temps (Loeber et Farrington, 2000 ; Tremblay, 2000). De plus, déterminer si des comportements sont violents renvoie à des considérations culturelles et à certaines divergences selon les milieux (Bouchard, Pelchat et Boudreault, 1996b).

\subsection{LES CARACTÉRISTIQUES INDIVIDUELLES, SOCIALES ET FAMILIALES}

Pour mieux saisir la problématique des comportements violents, il importe d'exposer certaines caractéristiques individuelles, sociales et familiales de l'enfant. Ces caractéristiques constituent des facteurs étroitement associés au risque de voir émerger de tels comportements. À cette fin, on accorde 
une attention toute particulière au sexe de l'enfant, au type de relations sociales qu'il entretient avec ses pairs, ainsi qu'au milieu familial dont il est issu.

\subsubsection{Les enfants ayant des comportements violents}

Le premier facteur de risque concerne le sexe de l'enfant. Ainsi, les garçons davantage que les filles font preuve de violence physique envers leurs pairs à l'école (Cloutier et Renaud, 1990; Day, Golench, MacDougall et Beals-Gonzales, 1995; Tremblay, Boulerice, Harden, McDuff, Pérusse, Pihl et Zoccolillo, 1996). Toutefois, lorsqu'il s'agit de violence verbale, les filles sont tout aussi enclines que les garçons, sinon davantage, à user de tels comportements (Loeber et Farrington, 2000; Tremblay, 2000).

Le tempérament de l'enfant constitue également un facteur de risque important (Baudier et Céleste, 2000; Turcotte, St-Amand, Beaudoin et Champoux, 2001), notamment par l'expression incontrôlée de la colère et de la frustration (Fabes, Eisenberg, Jones, Smith, Guthrie, Poulin, Shepard et Friedman, 1999 ; Tessier, Tarabulsy et Provost, 1996). On note également que l'impulsivité, l'humeur changeante et le degré d'activité élevé (Farrington, 2000; Hébert, 2001) sont des caractéristiques étroitement associées aux comportements violents.

Enfin, l'enfant aux prises avec le Trouble du déficit de l'attention avec hyperactivité (Juneau et Boucher, 2004), de même que celui ayant des difficultés d'apprentissage scolaire (Bender et Smith, 1990; Fortin, Toupin, Pauzé, Déry et Mercier, 1996; Mishna, 2003) est davantage qu'un autre porté à faire preuve de violence. De plus, les garçons du préscolaire qui présentent de hauts niveaux d'opposition face à l'adulte, de même que de l'hostilité, sont ceux qui ont le plus de risques de faire preuve de violence physique à l'adolescence (Nagin et Tremblay, 1999), voire à l'âge adulte (Kratzer et Hodgins, 1997).

\subsubsection{L'école, la garderie scolaire et les pairs}

L'entrée à la garderie et au préscolaire force les enfants à se conformer à de nouvelles exigences et à créer d'autres types de relations que celles qui leur sont familières dans leur milieu familial (Angelino et Meyer, 2001; Dumont, Provost et Dubé, 1990 ; Provost, 1990 ; Tessier, Tarabulsy et Provost, 1996). L'absence d'habiletés sociales entraîne parfois le harcèlement et le rejet de l'enfant par les pairs (Cowie, 2000; Evans et Scheuer, 1987).

Pris dans un cercle vicieux, beaucoup d'enfants harcelés et rejetés ont des comportements violents (Evans et Scheuer, 1987; Farmer, Farmer et Gut, 1999) ou, à l'inverse, sont eux-mêmes victimes de violence de la part des 
autres enfants (Evans et Scheuer, 1987). Quoi qu'il en soit, le harcèlement et le rejet poussent certains d'entre eux à se comporter de manière encore plus violente (Boivin, 1996) et à faire preuve d'inaptitude sociale (Dumont, Provost et Dubé, 1990). Il devient donc difficile de savoir si ce sont les comportements violents qui provoquent le harcèlement et le rejet ou si, à l'inverse, ce sont ceux-ci qui entraînent les comportements violents (Ladd, 1999). Les programmes axés sur le développement des compétences sociales chez les enfants (Bélanger, Bowen et Rondeau, 1999; Bowen, Rondeau, Rajotte et Bélanger, 2000; Couture, 2001 ; Royer, Morand, Desbiens, Moisan et Bitaudeau, 2000 ; Saint-Laurent, Giasson, Simard, Dionne et Royer, 1995; Turcotte, Lamonde et Lindsay, 2002) et sur l'intérêt accordé aux victimes reposent sur l'enjeu consistant à briser ce cercle de violence.

\subsubsection{Les parents et la famille}

Certains chercheurs croient que l'enfant ayant des comportements violents envers ses pairs provient généralement d'une famille qui favorise et encourage leur émergence (Schreiber et Schreiber, 2002; Tremblay, 2000). D'autres vont même jusqu'à affirmer que les enfants vivant dans une famille où la violence est présente au quotidien ont davantage de risques d'user de celle-ci (Loeber et Farrington, 2000; Loeber, Farrington, StouthhammerLoeber et Van Kammen, 1998). De récentes études ont également montré que ces parents ne possèdent pas toujours les compétences éducatives requises pour faire face à la situation (Akande, 2001; Cowie, 2000; Fontaine et Pourtois, 1998; Loeber, Farrington, Stouthhammer-Loeber et Van Kammen, 1998; Tremblay, 2000). Des chercheurs ont ainsi relevé, dans les différents styles parentaux de discipline tels qu'autoritaire, libéral-permissif et indifférent-désengagé, que certaines attitudes et pratiques éducatives sont de nature à encourager l'émergence de comportements violents chez l'enfant (Baumrind, 1991; Darling et Steinberg, 1993; Dumont, Provost et Dubé, 1990).

Chez le parent ayant un style autoritaire, il semble qu'une discipline trop stricte, punitive ou coercitive augmente la possibilité de voir émerger des difficultés de comportement (Schreiber et Schreiber, 2002; Steinberg, Elmen et Mounts, 1989). En réaction à un tel contrôle, l'enfant peut faire preuve de comportements violents pour se rebeller contre le parent ou pour se venger (Dumont, Provost et Dubé, 1990; Loeber, Farrington, Stouthhammer-Loeber et Van Kammen, 1998). 
Pour ce qui est des parents ayant un style libéral-permissif, caractérisé par le laisser-faire (Cavell, 2000 ; Vézina, Bradet, Lord, Pelletier et Thibault, 1995), l'enfant peut se sentir légitimé d'adopter des comportements violents puisque ceux-ci n'entraînent pas de conséquences fâcheuses (Cowie, 2000 ; Dumont, Provost et Dubé, 1990 ; Farrington, 2000).

Quant aux parents ayant un style indifférent-désengagé, qui utilisent de façon inconstante des méthodes disciplinaires, soit en ne faisant subir aucune conséquence à leur enfant suite à des comportements violents ou en le punissant trop sévèrement, ils accroissent le risque de voir ces comportements émerger à nouveau (Cavell, 2000 ; Vézina, Bradet, Lord, Pelletier et Thibault, 1995). À cet égard, « la constance dans le style parental d'éducation est considérée comme tout aussi importante, voire davantage, que la méthode éducative choisie» (McCain et Mustard, 1999, p. 104).

À l'inverse, plusieurs auteurs mentionnent que les parents ayant un style disciplinaire démocratique contribuent de façon positive à la socialisation de l'enfant (Baumrind, 1991; Darling et Steinberg, 1993; Deslandes et Royer, 1994 ; Potvin, Deslandes, Beaulieu, Marcotte, Fortin, Royer et Leclerc, 1999). Ce style encourage l'autonomie et la participation à toutes formes d'échanges, dans un mode de communication claire et bidirectionnelle. Dans pareil contexte, l'enfant reçoit un soutien émotionnel de ses parents et est exposé très tôt à des situations de socialisation qui lui permettent de composer avec les pairs de manière positive (Prêteur, Lescarret et de Léonardis, 1998).

Un autre facteur de risque, soit le manque de supervision parentale, est étroitement associé à l'émergence de comportements violents chez l'enfant (Cowie, 2000 ; Farrington, 2000 ; Loeber, Farrington, StouthhammerLoeber et Van Kammen, 1998). La supervision parentale consiste à assurer un contrôle et vérifier la bonne marche d'une tâche, d'un travail ou d'une activité réalisés par leur enfant. Le fait qu'il ne soit pas toujours possible pour ces parents d'assurer la qualité de la supervision parentale peut faire en sorte qu'il soit très ardu de régler la situation-problème (Kinnear, 1995). Face aux échecs répétés, certains d'entre eux finissent par se sentir impuissants et désespérés (Cavell, 2000).

La monoparentalité constitue également un facteur de risque très important associé à l'émergence de comportements violents chez l'enfant (Turcotte, St-Amand, Beaudoin et Champoux, 2001). À cet égard, Nagin et Tremblay (2001) avancent même que la faible scolarité des mères monoparentales, de même que leur jeune âge, de surcroît si elles ont donné naissance à leur enfant à l'adolescence, constituent des facteurs de risque indissociables de cette problématique. Cette situation est particulièrement observable chez les jeunes mères de familles monoparentales ayant un fils au préscolaire. 
En somme, beaucoup d'enfants ayant des comportements violents proviennent de familles vivant en contexte de pauvreté (Farrington, 2000; Pearl, 1987). Ce milieu socioéconomique est même reconnu comme étant celui qui génère le plus d'enfants aux prises avec une telle problématique (Cavell, 2000 ; Centre national d'information sur la violence dans la famille, 1997; Farrington, 2000; Kinnear, 1995; Loeber et Farrington, 2000). Par ailleurs, l'Enquête longitudinale canadienne sur les enfants et les jeunes met en lumière que, pour ce qui est des enfants ayant des difficultés comportementales, «le facteur le plus déterminant ne se limite pas à la classe sociale et au revenu des familles, mais concerne davantage le style parental d'éducation» (McCain et Mustard, 1999, p. 103). Notamment, les pratiques éducatives parentales qui s'avèrent constantes semblent être un facteur de protection très important contre le risque de voir émerger chez l'enfant des comportements violents envers ses pairs au préscolaire et au primaire (Landy et Tam, 1996).

Enfin, la majorité des parents d'enfants qui ont des comportements violents vivent de manière isolée socialement (Kinnear, 1995; Martichoux, 2000). Le faible soutien social constitue un autre facteur de risque important associé aux difficultés vécues par les enfants (Landy et Tam, 1996). En outre, les programmes de soutien à la parentalité (Duhamel-Maples, 1996) et au développement de compétences éducatives parentales (Paquin, Papillon et Rousseau, 1996) se basent sur cet état des connaissances.

Bref, en dépit du nouvel intérêt des médias face à la violence dans les écoles, il demeure primordial de mieux la comprendre afin de mieux la prévenir. Il importe également de privilégier la prévention et l'intervention précoces afin d'augmenter les chances de succès d'une telle entreprise. Un aspect peu exploré jusqu'à maintenant consiste en une meilleure compréhension de ce que vivent les parents, soit l'influence, voire le contrôle qu'ils exercent sur la situation-problème de leur enfant à l'école. Toutefois, avant d'aborder les nouveaux enjeux sous-jacents à cette problématique, un regard s'impose sur le cadre légal inhérent au contrôle de la violence dans les écoles.

\section{LE CADRE LÉGAL FACE À LA VIOLENCE DANS LES ÉCOLES}

La deuxième partie vise à mieux comprendre le contexte ayant mené à la refonte des lois canadiennes touchant la jeunesse qui ont été récemment adoptées dans le but de mieux faire face au phénomène de la violence. Elle aborde également la question de la Politique de tolérance zéro du ministère ontarien de l'Éducation, du Code de conduite provincial et des mesures 
disciplinaires sous-jacentes, de même que la Loi sur la sécurité dans les écoles permettant de contrer précocement l'émergence de comportements violents chez les enfants envers leurs pairs. Ce cadre légal est intimement lié au contexte de la présente étude en raison des nouvelles mesures, lois et règlements que doit dorénavant appliquer le milieu scolaire.

\subsection{LA REFONTE DES LOIS CANADIENNES TOUCHANT LA JEUNESSE}

La série d'événements tragiques survenus au cours des dernières années, notamment à Columbine aux États-Unis et à Erfurt en Allemagne, où des adolescents ont eu recours à des armes à feu pour régler leurs comptes avec des pairs et des enseignants, a forcé les autorités du monde entier à réfléchir à la manière de mieux les contrer (Lemonick, 2002). Les échecs des nombreux efforts consentis pour éviter la répétition de tels drames ont conduit naturellement à l'idée de la prévention et de l'intervention précoces des comportements violents. Pour appuyer cette position, les résultats de recherches longitudinales montrent que les comportements des enfants représentent de précieux indicateurs quant à la manière probable dont ils agiront à l'adolescence (Nagin et Tremblay, 2001 ; Rich, 1992; Tremblay, 1995) et même à l'âge adulte (Kratzer et Hodgins, 1997).

Le Canada n'a pas échappé à cette prise de conscience puisque, selon Viau (2001), au cours de la dernière décennie, il affichait le taux de mises sous garde d'adolescents délinquants le plus élevé de tous les pays occidentaux, y compris les États-Unis. Notamment, dans le but de faire une distinction entre le grand nombre d'infractions mineures commises par des adolescents et le petit nombre d'infractions graves avec violence, le gouvernement canadien procédait, en 1998, à la refonte de la Loi sur les jeunes contrevenants (Ministère de la Justice du Canada, 1998). Puis, en 2002, il procédait à l'adoption de la nouvelle Loi sur le système de justice pénale pour les adolescents (Ministère de la Justice du Canada, 2002). Cette nouvelle loi fédérale donne notamment au tribunal pour adolescents le pouvoir d'imposer des peines d'adulte de plus de deux ans d'emprisonnement dès l'âge de 14 ans, soit deux ans de moins que l'âge qui était requis auparavant. 


\subsection{La Politique de tolérance zéro DU MINISTĖRE ONTARIEN DE L'ÉDUCATION}

En Ontario, l'intervention face à la violence dans les écoles signifiait déjà de se conformer à la Politique de tolérance zéro du ministère de l'Éducation et de la Formation (1994). De cette politique découlait l'obligation pour les écoles de la province de se doter d'un code de conduite prévoyant les diverses conséquences face aux comportements violents des élèves.

\subsection{LE CODE DE CONDUITE DES ÉCOLES ET LEURS MESURES DISCIPLINAIRES}

Les normes de gravité établies par le code de conduite des écoles correspondent généralement à celles de la Conflict Tactics Scale (Straus et Gelles, 1990). Cette échelle américaine détermine notamment deux catégories et sous-catégories de comportements violents, soit les verbaux et les physiques, mineurs et graves. À titre d'exemple, le comportement violent d'ordre physique le plus grave et le plus fréquent aux États-Unis, qui justifie la suspension scolaire externe chez les enfants de 3 à 9 ans, est celui de frapper un pair avec force et blessures, soit de donner un coup de pied, un coup de poing ou de mordre (Dupper et Bosch, 1996).

La situation qui prévaut dans les écoles américaines est très comparable à celle du Canada (Vitaro et Gagnon, 2000). Dans la province de l'Ontario, pour de tels comportements violents, la suspension scolaire externe est autorisée jusqu'à concurrence de vingt jours ouvrables au cours d'une même année (Ministère de l'Éducation et de la Formation de l'Ontario, 1994). Au-delà de ce nombre, le renvoi de l'école peut être envisagé pour une période d'une année si aucun changement n'est observé suite à des interventions répétées auprès de l'enfant et de sa famille (Ministère de l'Éducation de l'Ontario, 2000). Le renvoi débute généralement vers l'âge de 9 ans et implique un changement d'école. Actuellement, les élèves peuvent faire face à deux types de renvoi: partiel ou complet. Les élèves faisant face à un renvoi partiel de leur école font généralement l'objet d'un placement dans des écoles et des classes spéciales, aux termes de la Section 20 de la Loi sur l'éducation de l'enfance en difficulté (Ministère de l'Éducation de l'Ontario, 2001b). Quant aux élèves faisant face à un renvoi complet de toutes écoles de l'Ontario financées par des fonds publics, ils ont l'obligation depuis peu de s'acquitter avec succès d'un programme de discipline rigide (Ministère de l'Éducation de l'Ontario, 2001e) afin d'y être admis à nouveau. Pour répondre à ce nouveau besoin, une quinzaine 
d'écoles axées sur un tel programme disciplinaire, que l'on surnommait anciennement "écoles de réforme», ont été implantées à titre expérimental dans toutes les régions de l'Ontario.

\subsection{Le Code DE CONDUITE PRovincial}

Parallèlement à cette situation, dans le but de mieux encadrer le code des écoles, le ministère de l'Éducation de l'Ontario adoptait, en 2001, le Code de conduite provincial (Ministère de l'Éducation de l'Ontario, 2001a). Ce code, en plus d'élaborer des normes claires et uniformes pour toute la province, vise à assurer la sécurité de toute personne se trouvant dans une école. Il rend dorénavant obligatoire et immédiate la suspension scolaire externe, non seulement dans des cas de violence physique, mais également dans le cas de violence verbale, consistant notamment à menacer de causer des dommages corporels graves. À cette mesure s'ajoute le nouveau pouvoir des directions d'écoles et du personnel enseignant de suspendre ou de renvoyer les élèves dans le cas d'infractions graves, notamment en raison d'une agression physique causant des dommages corporels à autrui et nécessitant les soins d'un professionnel de la santé. L'adoption du Code de conduite provincial forçait ainsi les écoles de l'Ontario à réviser leur propre code de conduite, en vue de le rendre conforme aux nouvelles normes émises par le ministère de l'Éducation (Ministère de l'Éducation de l'Ontario, 2001a).

\subsection{La LoI SUR La SÉCURITÉ dANS Les ÉCOLES de L’ONTARIO}

Préalablement à l'adoption du Code de conduite provincial, dans la foulée du mouvement de durcissement des politiques et des programmes fédéraux visant la prévention et l'intervention précoces des comportements violents, l'Ontario procédait à l'adoption de la nouvelle Loi sur la sécurité dans les écoles (Ministère de l'Éducation de l'Ontario, 2000). Cette nouvelle loi autorise l'application de mesures coercitives et dissuasives dès l'apparition, chez les enfants, des premiers comportements jugés inappropriés en milieu scolaire. Parmi ces mesures, la suspension scolaire externe peut dorénavant être appliquée dès l'âge de 3 ans.

En définitive, c'est dans ce nouveau cadre légal, tant fédéral que provincial, que les écoles ontariennes doivent dorénavant envisager la gestion des comportements violents. C'est également dans ce contexte que le milieu scolaire risque fort, au cours des prochaines années, de connaître un phénomène de recrudescence de la suspension scolaire externe en réponse aux comportements violents des enfants de 3 à 9 ans. Ce cadre 
légal n'est pas sans causer un profond questionnement quant aux effets de l'application d'une telle mesure disciplinaire sur le processus de scolarisation et de socialisation des élèves du préscolaire et du primaire. De plus, cette situation invite les parents à mieux s'entendre avec l'école, tant sur la manière que sur les modalités qu'implique l'application des nouvelles lois, règlements et politiques en matière de contrôle de la violence dans les écoles de l'Ontario. À ce sujet, comment les parents peuvent-ils être amenés à mieux collaborer avec l'école en vue de trouver des solutions à la situation-problème de leur enfant au préscolaire et au primaire? Quel type de collaboration devraient-ils privilégier? Quelles sont les différentes conceptions que l'école entretient au sujet de la collaboration avec les parents? Bref, quels sont les facteurs de soutien et les obstacles sousjacents à une bonne collaboration entre les milieux scolaire et familial, dans une démarche d'empowerment parental? Dans le prochain chapitre, nous proposons plusieurs pistes de réponses en fonction des plus récents écrits sur ces questions. 



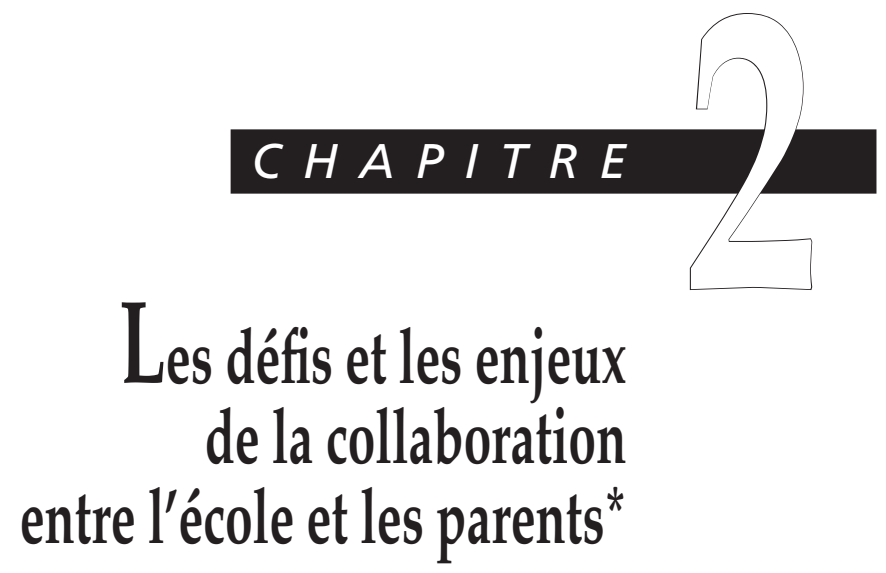

* Des extraits de ce chapitre sont tirés de: M. Paquin et M. Drolet (2005).«Travail de collaboration entre les parents et les autres intervenants", dans L. Massé, N. Desbiens et C. Lanaris (dir.), Les troubles de comportement à l'école: prévention, évaluation et intervention, Boucherville, Gaëtan Boucher éditeur, p. 297-307. 

Le deuxième chapitre de cet ouvrage porte sur les défis et les enjeux de la collaboration entre l'école et les parents. Dans un premier temps, nous présentons les définitions portant sur la collaboration ainsi que celles des concepts qui lui sont étroitement associés. Nous posons un regard sur les types de collaboration par rapport aux différentes conceptions qu'entretient l'école, dans le contexte propre au milieu scolaire franco-ontarien. En vue d'établir un état de la situation au sujet de la collaboration entre l'école et les parents, nous abordons diverses perspectives dans le temps ainsi que les nouvelles réalités familiales. Ce regard nous amène à identifier les facteurs de soutien et les obstacles sous-jacents à la collaboration entre l'école et les parents, tant sous l'angle des familles que par rapport aux perceptions qu'ils entretiennent face au milieu scolaire. Nous cernons ensuite le concept $\mathrm{d}^{\prime}$ empowerment parental en fonction des gestes d'adaptation, d'opposition et de résistance que les parents posent afin d'exercer une plus grande influence, voire un meilleur contrôle, sur la situation-problème de leur enfant à l'école.

\section{LE CONCEPT DE COLLABORATION}

Cette première partie vise à mieux comprendre le concept de collaboration entre l'école et les parents et les concepts qui lui sont étroitement associés. Par la suite, nous présentons deux typologies d'implication parentale dans la vie scolaire de l'enfant afin de jeter un éclairage sur différents aspects de la collaboration. Diverses conceptions qu'entretient le milieu scolaire à l'endroit des parents font également l'objet d'une attention particulière puisqu'elles rendent parfois la collaboration encore plus complexe. À cet effet, nous cernons le contexte propre au milieu scolaire franco-ontarien puisque l'échantillon de la présente recherche est essentiellement composé de parents d'enfants francophones qui habitent la région d'Ottawa. Finalement, nous positionnons la présente recherche par rapport aux principaux écrits qui ont été publiés sur le sujet ces dernières années.

\subsection{LES DÉFINITIONS DE LA COLLABORATION ET DES CONCEPTS ASSOCIÉS}

Plusieurs concepts sont étroitement associés à celui de collaboration entre l'école les parents, soit l'interaction ou la relation entre les milieux scolaire et familial, la participation ou l'implication parentales, la coopération ainsi que le partenariat entre l'école et la famille. La pluralité des définitions pour chacun d'eux, un manque de consensus entre les auteurs quant à leur nature et leur portée ainsi qu'un usage commun de tous ces termes, 
comme s'il s'agissait de concepts interchangeables, nuisent à leur bonne compréhension. Afin de bien saisir ce que signifie la collaboration entre l'école et les parents, il importe de lier les définitions aux différentes conceptions qui prévalent dans le monde de l'éducation.

Depuis une dizaine d'années, les notions de collaboration ou de partenariat ont supplanté celles de relation, de participation ou d'implication parentales (Epstein et Sanders, 1997; Gareau et Sawatsky, 1995; Kay, Fitzgerald et McConaughy, 2002; Montandon et Perrenoud, 1994; Pelco, Jacobson, Ries et Melka, 2000). Le terme collaboration entre les milieux scolaire et familial est même devenu l'un des plus importants dans le discours politique des dernières années (Conseil de la famille et de l'enfance du Québec, 2000; Ministère de l'Éducation du Québec, cité dans Deslandes, 1999; Ministère de l'Éducation de l'Ontario, 2001a, 2001b; Organisation de coopération et de développement économiques, 1997). Cette évolution n'est pas qu'un changement de termes; elle traduit différentes conceptions sous-jacentes.

Selon Christenson et Sheridan (2001, p. 95), la collaboration est définie comme étant «un cadre de travail dynamique qui implique une forme d'interaction collégiale, interdépendante et égalitaire entre les éducateurs et les familles, leur permettant de travailler ensemble pour atteindre des buts communs». Une bonne collaboration se traduit par la mise en place $d^{\prime}$ 'une communication bidirectionnelle et d'un soutien mutuel, ainsi que par des prises de décisions communes. Lors d'une négociation entre l'école et les parents, les deux milieux s'engagent à maintenir la relation positive. Toujours selon ces auteurs, les trois caractéristiques associées à une bonne collaboration sont la compréhension des contraintes de toutes les parties en cause, l'absence de rigidité dans les rôles et les responsabilités, ainsi que le maintien de frontières claires. Une écoute active des préoccupations de chacun s'avère cruciale. Le succès d'une telle entreprise repose sur la confiance mutuelle que développent les interlocuteurs, fondée sur une qualité de communication et le respect de leurs compétences (Adams et Christenson, 2000).

D'autres auteurs apportent entre tous ces termes des distinctions qu'ils empruntent aux sciences administratives (Bouchard, Clarkson et Tessier, 1996; Boutin et Le Cren, 1998; Dunst, Trivette et Johanson, 1994; Gareau et Sawatsky, 1995). Selon Bouchard, Clarkson et Tessier (1996, p. 23), la collaboration signifie «la participation à la réalisation d'une tâche ou la prise en charge d'une responsabilité sans faire intervenir de condition de réciprocité dans le partage de celles-ci». Quant à Deslandes (2001, p. 256), elle la décrit plutôt comme étant le « rôle des parents dans l'éducation de leur enfant à l'école et à la maison ». La participation parentale, qu'elle soit 
sur un plan individuel ou collectif, devient alors une situation permettant d'établir une collaboration, puis de mettre en place un partenariat avec l'école (Deslandes, 1999).

Pour Claes et Comeau (1996), il y a deux principales significations au terme "participation». Selon eux, dans un sens plus limité, participer veut dire que les parents s'informent de l'école et de ses programmes, alors que dans son sens le plus large, il consiste à ce qu'ils prennent part aux décisions et collaborent de façon active à la réalisation d'objectifs. Entre ces deux réalités, il y a des situations où la participation des parents signifie pour eux de s'engager, de coopérer, ou de s'associer à des projets et à des activités scolaires (Comeau et Salomon, 1994).

Epstein (1992) et l'Organisation de coopération et de développement économiques (1997) mettent plutôt en valeur la notion de partenariat, qui désigne une alliance formelle entre les deux milieux, leur permettant de travailler conjointement à l'atteinte de buts communs. Le partage de responsabilités est alors mis en évidence entre l'école et la famille, où des membres autres que les parents deviennent des partenaires potentiels (Epstein, 1992).

Pour Bouchard, Clarkson et Tessier (1996, p. 24), le partenariat se définit par «l'association de personnes qui reconnaissent leurs expertises et leurs ressources mutuelles, ainsi que par l'égalité dans la prise de décisions consensuelles entre les partenaires». Cette définition accorde une grande importance au pouvoir décisionnel que partagent les partenaires que sont les enseignants, les directions d'école et les parents. Deslandes (1999) soutient toutefois que le vrai partenariat suppose une confiance mutuelle et une communication bidirectionnelle, en plus d'un effet de réciprocité dans un rapport fondé sur le respect.

Par ailleurs, dans le domaine de la relation d'aide, Dunst, Trivette et Johanson (1994) définissent le partenariat entre un professionnel et un parent comme un travail de collaboration visant la poursuite de buts communs. Le partenariat se caractérise par des contributions mutuelles, une entente quant aux rôles de chacun, un désir de travailler ensemble vers l'atteinte de buts communs, un partage des responsabilités vis-à-vis des actions à entreprendre, ainsi que le développement d'une confiance mutuelle basée sur l'échange d'information. Dans ce type de relation, la responsabilité des décisions concernant l'enfant est assumée par les parents. Le partenariat donne à ces derniers l'occasion de devenir compétents dans les zones où se déroule le travail de collaboration. "La participation active des partenaires est requise dans le but de rehausser leur sentiment de contrôle et d'efficacité» (Dunst, Trivette et Johanson, 1994, p. 201). De plus, elle donne aux parents des occasions de poser des gestes 
d'empowerment, soit la perception d'exercer une influence, voire un contrôle sur la situation-problème de leur enfant à l'école. Cette perspective se fonde sur les compétences éducatives que possèdent déjà les parents et sur celles qu'ils développent tout au long de la relation d'aide.

Dans les écrits sur le sujet, il est également question de partenariat collaboratif ou associatif «lorsqu'il s'agit pour le parent de réaliser une tâche ou une responsabilité en réponse à l'invitation de l'école et des enseignants» (Deslandes, 1999, p. 3).

Enfin, seule la coopération semble se démarquer clairement des autres termes puisqu'elle se limite soit à un partage des tâches entre les parties (Bouchard, Clarkson et Tessier, 1996; Gareau et Sawatsky, 1995), soit au cadre des activités scolaires (Comeau et Salomon, 1994).

\subsection{Les tYPES DE COLLABORATION}

Toutes les définitions associées à la collaboration traduisent différentes manières pour les parents d'assurer leur présence dans la vie scolaire de leur enfant (Grolnick, Benjet, Kurowski et Apostoleris, 1997; Grolnick et Slowiaczek, 1994; Royer, Saint-Laurent, Bitaudeau et Moisan, 1995). La présence parentale se manifeste également par différentes formes d'implication (Comeau et Salomon, 1994; Reynolds, 1992). Afin de saisir la complexité du concept d'implication parentale et la variété des situations possibles, des typologies ont été élaborées, notamment par Epstein (1995) et Gordon (1996, cité dans dans Claes et Comeau, 1996).

La typologie la plus connue et la plus citée est celle d'Epstein (1995). Elle a fait école aux États-Unis en permettant d'établir des normes régissant les programmes américains d'implication parentale (National PTA, 2000, dans Christenson et Sheridan, 2001). À ce titre, l'auteure propose six types d'implication parentale dans la vie scolaire de l'enfant. Chacun des six types d'implication correspond à une forme de collaboration entre l'école et les parents. De plus, elle identifie des zones où l'école reconnaît et soutient l'apport des parents sur le plan individuel ou collectif.

Dans le type 1 (parenting), les compétences éducatives parentales font l'objet d'une promotion et sont soutenues par l'école, qui aide les parents à comprendre le développement de l'enfant et à lui fournir un environnement familial favorable à sa scolarisation et à sa socialisation. Cet aspect comprend l'influence de la famille sur le rendement scolaire, influence qui fait notamment appel aux conditions matérielles de vie. Dans le type 2 (communicating), la communication entre l'école et les parents est régulière, bidirectionnelle et significative. De plus, elle est basée sur la confiance et le soutien mutuels. Il s'agit principalement pour l'école 
d'informer les parents des programmes scolaires et des progrès de l'enfant. Lors de rencontres, il y a échange d'informations qui servent parfois à mettre en place un processus de résolution de problèmes. Dans le type 3 (volunteering), les parents sont les bienvenus à l'école. Leur aide et leur soutien sont désirés. Les parents sont notamment invités à participer aux activités de bénévolat organisées par l'école. Il peut s'agir d'un soutien aux levées de fonds ou à l'organisation d'activités se déroulant en classe, ou encore d'un accompagnement aux sorties scolaires. Dans le type 4 (learning at home), les parents jouent un rôle important à la maison. Ils sont invités à compléter le rôle de l'enseignant, notamment en assistant leur enfant pendant la période de devoirs et de leçons ou en discutant quotidiennement avec eux de sujets en relation avec les apprentissages scolaires. En ce qui a trait au type 5 (decision making and advocacy), les parents sont considérés comme des partenaires à part entière dans le processus de prise de décisions concernant leur enfant et leur famille. De plus, ils peuvent participer aux décisions touchant l'institution scolaire par le biais de comités. Finalement, dans le type 6 (collaborating with community), l'école est présente dans la communauté en mettant ses ressources au service de celle-ci. Ce type d'implication correspond à la volonté de l'école de s'intégrer à la communauté en offrant des services de soutien tant aux enfants qu'aux parents, ainsi qu'en rendant ses infrastructures accessibles aux organismes à l'extérieur des heures de classe, afin de leur permettre d'y tenir des activités de loisir ou autres. L'école fait partie intégrante de la communauté en y devenant la plaque tournante (Martin, Tett et Kay, 1999). La typologie de l'implication parentale d'Epstein (1995) est présentée au tableau 2.1.

\section{TABLEAU 2.1}

\section{La typologie de l'implication parentale d'Epstein (1995)}

1. Compétences éducatives parentales (parenting). Elles font l'objet d'une promotion et sont soutenues par l'école.

2. Communication entre l'école et les parents (communicating). Elle est régulière, bidirectionnelle et significative.

3. Activités de bénévolat (volunteering). Les parents sont les bienvenus à l'école. Leur aide et leur soutien sont désirés.

4. Apprentissage à la maison (learning at home). Les parents jouent un rôle important à la maison en assistant leur enfant pendant la période de devoirs et de leçons.

5. Prise commune de décisions (decision making and advocacy). Les parents sont considérés comme des partenaires à part entière dans le processus de prise de décisions concernant leur enfant et leur famille.

6. Collaboration avec la communauté (collaborating with community). L'école est présente dans la communauté et met ses ressources au service de celle-ci. 
Une autre typologie, cette fois élaborée par Gordon (1996, cité dans Claes et Comeau, 1996), illustre divers apports possibles des parents à la vie scolaire de leur enfant. Cette typologie peut reposer sur un modèle circulaire ou hiérarchique. Dans un modèle circulaire, tous les aspects d'investissement parental sont d'importance égale, tandis que dans un modèle hiérarchique, ils sont progressifs, allant de l'information jusqu'à la prise de décisions. Les activités officielles de l'école, soit les rencontres entre les enseignants et les parents, notamment lors de la remise des bulletins, constituent un prérequis à toute autre forme $\mathrm{d}$ 'investissement parental. Des rôles spécifiques sont attribués à chaque type d'investissement parental.

Dans le type 1, le parent joue son rôle d'éducateur en s'informant auprès de son enfant de ses apprentissages scolaires et en lui apportant son soutien à la maison. Dans le type 2, le parent joue un rôle d'auditeurobservateur en informant les membres de la communauté des activités scolaires. Dans le type 3, le rôle du parent est celui d'un bénévole. Il offre de la surveillance à l'école que fréquente son enfant et accompagne sa classe lors des sorties scolaires. Dans le type 4, le parent joue un rôle paraprofessionnel qui offre son soutien à l'enseignant en participant à l'organisation et à la réalisation d'activités pédagogiques en classe. Finalement, dans le type 5, le parent joue un rôle de décideur en participant aux prises de décisions et en coopérant à la formulation et à la réalisation de projets et d'objectifs communs. La typologie de l'investissement parental de Gordon (1996, cité dans Claes et Comeau, 1996) est présentée au tableau 2.2.

\section{TABleAu 2.2}

\section{La typologie de l'investissement parental de Gordon \\ (1996, cité dans Claes et Comeau, 1996)}

1. Le parent éducateur s'informe auprès de son enfant de ses apprentissages scolaires et lui apporte son soutien à la maison.

2. Le parent auditeur-observateur informe les membres de la communauté des activités scolaires.

3. Le parent bénévole offre de la surveillance à l'école que fréquente son enfant et d'accompagner sa classe lors des sorties scolaires.

4. Le parent paraprofessionnel offre son soutien à l'enseignant en participant à l'organisation et à la réalisation d'activités pédagogiques en classe.

5. Le parent décideur participe aux prises de décision et coopère à la formulation et à la réalisation de projets et d'objectifs communs.

Les typologies d'Epstein (1995) et de Gordon (1996, cité dans Claes et Comeau, 1996) illustrent le caractère multidimensionnel de l'implication et de l'investissement parental. Des nuances ont été apportées à ces 
deux typologies au cours des dernières années afin de s'assurer qu'elles donnent lieu à une bonne collaboration entre les milieux scolaire et familial. Toutefois, à l'image des multiples formes que peuvent prendre tant l'implication que l'investissement parental, chaque conception de l'école génère sa propre forme de collaboration avec les parents.

\subsection{LES CONCEPTIONS DE L'ÉCOLE AU SUJET DE LA COLLABORATION AVEC LES PARENTS}

L'école entretient différentes conceptions au sujet de la collaboration avec les parents. Notamment, elle peut leur imposer une vision hiérarchique ou participative du rôle qu'ils doivent jouer dans la collaboration (Claes et Comeau, 1996). Dans une vision hiérarchique, l'école est l'unique détentrice de l'autorité. Cette situation implique qu'elle se sent légitimée de contribuer à l'éducation du parent. Ce dernier est invité à collaborer essentiellement dans l'optique de faciliter la tâche de l'enseignant. On peut également le consulter pour certaines décisions à prendre concernant l'enfant et l'inviter à participer à des activités scolaires, mais l'école demeure le maître d'œuvre. À l'inverse, dans une vision participative, l'école encourage le développement de relations égalitaires entre l'enseignant et le parent. Elle favorise l'implication parentale dans les apprentissages scolaires de l'enfant ainsi que la participation «au choix et à la réalisation des priorités d'action» (Comeau et Salomon, 1994, p. 208).

Montandon et Perrenoud (1994) cernent diverses conceptions et attitudes que l'école entretient au sujet de la collaboration avec les parents. Selon ces auteurs, à certaines occasions, l'école considère les parents comme des clients. Il importe de les informer, parfois même abondamment. Toutefois, cette transmission d'informations, même si elle s'effectue de façon bidirectionnelle, n'engendre pas nécessairement une communication si elle porte essentiellement sur des événements ou des activités ponctuels. Pour Marcon (1999), le fait de prendre régulièrement contact avec les parents et de les informer de manière authentique demeure le point de départ pour accroître leur participation au processus de scolarisation de leur enfant. En ce qui concerne les progrès scolaires, les écoles sont obligées d'en informer les parents, et ce, à au moins deux ou trois reprises pendant l'année scolaire, selon la Charte ontarienne des droits et devoirs associée à la Loi de l'éducation (Ministère de l'Éducation de l'Ontario, 2001a).

Par ailleurs, les parents sont parfois considérés par l'école comme des cautions. L'école consulte les parents pour obtenir leurs opinions, mais «aucune garantie ne leur est donnée que leurs idées ou leurs recommandations seront mises en application» (Montandon et Perrenoud, 1994, p. 38). 
Lorsque les parents exercent des pressions pour faire reconnaître leurs idées, individuellement ou en groupe de pression, l'école peut les percevoir comme des adversaires. Cette perception engendre souvent des réactions négatives ou même défensives vis-à-vis les revendications et les récriminations parentales. Finalement, l'école perçoit assez négativement les parents qui revendiquent le droit de participer aux décisions concernant leur enfant. Les parents, qui insistent sur l'importance de prendre part à de telles décisions, en plus d'être informés, inscrivent cette forme de collaboration dans un idéal démocratique (Organisation de coopération et de développement économiques, 1997). Toutefois, cette situation peut générer des conflits lorsque la position de l'école et celle des parents sont trop éloignées l'une de l'autre (Boutin et Le Cren, 1998). De tels affrontements font tout de même partie intégrante du processus de collaboration et peuvent parfois être porteurs de solutions. Mais Peña (2000) soutient que la participation aux prises de décisions est davantage symbolique puisqu'elle nuit plus souvent qu'autrement à l'harmonie, crée trop de tensions et fait éclater les conflits entre l'école et les parents.

\subsection{LE CONTEXTE PROPRE AU MILIEU SCOLAIRE FRANCO-ONTARIEN}

Au cours des dernières années, la collaboration entre l'école et les parents a fait l'objet de quelques prises de position de la part du ministère de l'Éducation de l'Ontario (2001a). En faveur de la participation parentale qui reflète les principales composantes de la typologie d'Epstein (1995), le Ministère met notamment en valeur l'appui aux devoirs et aux leçons, la communication au sujet des progrès scolaires de l'enfant par l'intermédiaire des rencontres de remise des bulletins, le bénévolat, la participation aux décisions en matière d'éducation et l'implication parentale dans les comités d'école. Cette position vise à ce que «les parents puissent exercer une plus grande influence sur l'éducation de leurs enfants. [...]. La Charte ontarienne des droits et devoirs en éducation prévoit même que chaque parent ait le droit d'être un partenaire actif dans le système d'éducation » (Ministère de l'Éducation de l'Ontario, 2001a, p. 1-2). Plus précisément, la mise sur pied de conseils d'école, soit des comités consultatifs formés de parents élus, a été rendue obligatoire en 1997. Ces conseils visent à leur donner «le droit de se faire entendre et de participer de manière active à la vie de leur école» (Ministère de l'Éducation de l'Ontario, 2001a, p. 1). Les règlements adoptés en 2001 légitiment la consultation des conseils d'école sur des «questions influençant l'apprentissage des élèves » (Ministère de l'Éducation de l'Ontario, 2001b, p. 3). À cet égard, le ministère de l'Éducation de l'Ontario a clarifié la fonction consultative et la responsabilité du système scolaire 
de la manière suivante: «Les conseils scolaires et les directions d'écoles doivent parfois demander l'opinion des conseils d'école. [...]. Par contre, ils doivent toujours tenir compte des conseils prodigués par les conseils d'école» (Ministère de l'Éducation de l'Ontario, 2001a, p. 1). En outre, ils "étudient les recommandations formulées par les conseils d'école et les informent des mesures qu'ils entendent prendre à la suite de leurs recommandations» (Ministère de l'Éducation de l'Ontario, 2001b, p. 3).

Dans le contexte propre au milieu scolaire franco-ontarien, afin de contrer les comportements violents de l'enfant au préscolaire et au primaire, il importe de spécifier la position du ministère de l'Éducation à l'égard de la collaboration entre l'école et les parents. Notamment, dans le Code de conduite provincial (Ministère de l'Éducation de l'Ontario, 2001c, p. 6-7), il est du devoir des parents «d'appuyer les efforts du personnel de l'école visant à maintenir un climat d'apprentissage sûr et respectueux pour tous les élèves». En plus de s'intéresser activement au travail scolaire et de communiquer régulièrement avec l'école, les parents «encouragent et aident leur enfant à suivre les règles de comportement, aident le personnel de l'école à traiter des questions de discipline».

Finalement, comme les comportements violents sont souvent associés aux difficultés d'apprentissage, selon la Politique sur l'enfance en difficulté (Ministère de l'Éducation de l'Ontario, 2001d), les parents sont également invités à participer activement à l'élaboration du Plan d'enseignement individualisé (PEI), de concert avec le personnel scolaire.

\subsection{LE POSITIONNEMENT DE LA PRÉSENTE RECHERCHE}

La présente recherche s'inscrit dans la foulée des écrits de Christenson et Sheridan (2001). Dans ce contexte, la notion de collaboration entre l'école et les parents renvoie au travail conjoint effectué par les milieux scolaire et familial afin de contrer les comportements violents chez les 3 à 9 ans. La collaboration suppose une communication bidirectionnelle, un soutien mutuel et la prise de décisions communes concernant l'enfant. Ce travail signifie qu' une participation active des parents est requise à l'école. Leurs compétences étant reconnues, ceux-ci bénéficient d'occasions leur permettant de poser des gestes d'empowerment afin d'œuvrer à l'établissement d'un partenariat fondé sur la réciprocité.

La typologie d'Epstein (1995), plus particulièrement celle qui a été adaptée par les National Standards for Parents / Family Involvement Programs (National PTA, 2000 dans Christenson et Sheridan, 2001), est également celle que nous retenons, puisqu' elle repose sur six types reconnus d'implication parentale dans la vie scolaire de l'enfant. 
Par ailleurs, afin que se concrétise la participation active des parents dans le processus de prise de décisions concernant leur enfant à l'école, Montandon et Perrenoud (1994) soutiennent que des modifications doivent s'opérer dans les rapports entre le système scolaire et familial. Ces modifications concernent notamment une plus grande ouverture de la part du personnel scolaire face à l'apport parental. Une attention particulière y est accordée lors de l'analyse des données touchant les gestes d'empowerment parental.

Finalement, dans leur définition du partenariat entre l'école et les parents, Pourtois et Desmet (1997, p. 257) insistent sur une «tâche difficile à réaliser, toujours à redéfinir mais possible et prometteuse grâce à une intersubjectivité claire et voulue entre des partenaires». C'est pourquoi la question de la volonté, tant de l'école que des parents, de s'investir dans une collaboration efficace et fructueuse fait également l'objet $d^{\prime} u n e$ attention particulière lors de l'analyse des données.

\section{L'ÉTAT DE LA COLLABORATION}

Cette partie vise à faire état de la collaboration entre l'école et les parents. Nous y cernons d'abord les bienfaits de cette collaboration, notamment chez les enfants de 3 à 9 ans ayant des comportements violents envers leurs pairs au préscolaire et au primaire. Nous traitons ensuite du concept de collaboration dans une perspective historique. Puis, les nouvelles réalités des familles sont mises en lumière. Par la suite, nous précisons les facteurs de soutien et les obstacles sous-jacents à la collaboration entre l'école et les parents, en fonction de certaines variables sociodémographiques telles que le milieu socioéconomique et l'appartenance ethnoculturelle des parents. La présente partie se termine par un regard posé sur certaines considérations affectant la collaboration entre l'école et les parents sous l'angle du milieu scolaire.

\subsection{Pourquol collaborer?}

Selon beaucoup d'auteurs, la collaboration entre l'école et les parents est intimement liée à la réussite scolaire des élèves (Centre de recherche et d'intervention sur la réussite scolaire, 1993; Fortin et Mercier, 1994; Keith, Keith, Quirk, Sperduto, Santillo et Killings, 1998; Kohl, Lengua et McMahon, 2000; Organisation de coopération et de développement économiques, 1997; Peña, 2000 ; Reynolds, 1999; Royer, Saint-Laurent, Bitaudeau et Moisan, 1995; Saint-Laurent, Royer, Hébert et Tardif, 1994; Salomon et Comeau, 1998; Zellman et Waterman, 1998). Dans ce contexte, 
elle semble favoriser positivement le développement social des enfants du préscolaire et du primaire (Conseil de la famille et de l'enfance du Québec, 2000; Grolnick, Benjet, Kurowski et Apostoleris, 2002; Pourtois et Desmet, 1997; Royer, Morand, Desbiens, Moisan et Bitaudeau, 2000). Elle représente également un facteur de protection pour les élèves à haut risque d'échec et d'abandon scolaires (Centre de recherche et d'intervention sur la réussite scolaire, 2001; Potvin, Deslandes, Beaulieu, Marcotte, Fortin, Royer et Leclerc, 1999; Royer, Moisan, Saint-Laurent, Giasson et Boisclair, 1992), de même qu'un «filet de sécurité pour promouvoir l'apprentissage chez les enfants» (Christenson et Sheridan, 2001, p. 7). Pour sa part, le ministère de l'Éducation de l'Ontario (2001a, p. 1) avance que «les résultats des élèves sont meilleurs lorsque les parents s'intéressent à l'éducation de leurs enfants et $s^{\prime} y$ impliquent activement». La participation des parents au sein de l'école, notamment au niveau de la maternelle, permet aux enfants de mieux s'ajuster au monde scolaire (Deslandes et Jacques, 2004 ; Reynolds, Weissberg et Kasprow, 1992; Salomon et Comeau, 1998). Cet ajustement leur permet plus tard d'être davantage motivés (Gaziel et Warnet, 1996; Grolnick, Gurland, Jacob et Decourcey, 2002; Viau, 1999), voire de mieux performer (Normandeau et Nadon, 2000). De plus, elle a des effets positifs sur le développement de compétences sociales des enfants (Kohl, Lengua et McMahon, 2000) en diminuant leurs problèmes comportementaux (Algozzine et Kay, 2002; Burke, Loeber et Birmaher, 2002; Evans, Okifuji, Engler et Bromley, 1993; Goupil, 1997a; Reid, Eddy, Fetrow et Stoolmiller, 1999; Reynolds, 1999; Sheridan, Kratochwill et Elliott, 1990; Webster-Stratton, 1993). La collaboration entre l'école et les parents permet également aux enfants de développer une attitude plus positive à l'égard de l'école et une plus grande assiduité (Claes et Comeau, 1996; Deslandes, 2001). Développées tôt, de telles attitudes et valeurs peuvent avoir des répercussions chez les adolescents en accroissant leur désir de réussite scolaire (Paulson, 1994), en développant chez eux une plus grande persévérance aux études, voire en leur permettant d'accéder à des niveaux scolaires plus élevés (Reynolds et Walberg, 1992).

Les parents qui collaborent éprouvent généralement un plus grand sentiment de compétence éducative et entretiennent une meilleure communication avec leur enfant (Akkari, 1999; Peña, 2000). Bref, la collaboration bénéficie non seulement aux enfants mais également aux parents, en augmentant leur satisfaction envers l'école et leur confiance envers les enseignants (Dye, 1989).

Chez les enseignants, la collaboration avec les parents leur permet de développer une attitude plus positive envers ces derniers et envers leur pratique professionnelle (Gohier, 1996). Ils gagnent également un sentiment d'efficacité personnelle et une plus grande satisfaction professionnelle 
(Ozer et Bandura, 1990). Bref, les enseignants qui réussissent à intéresser les parents à l'école éprouvent des sentiments davantage positifs à l'égard de leur travail et ont moins tendance à considérer les parents incompétents, notamment ceux provenant de groupes socioéconomiquement défavorisés (Christenson et Sheridan, 2001; Epstein, 2001).

Il y a toutefois lieu de nuancer quelque peu tous ces constats positifs en raison du caractère multidimensionnel de l'implication parentale ainsi que des divergences de points de vue constatées dans les différentes études consultées (Feuerstein, 2000; Georgiou, 1999; Keith, Keith, Quirk, Sperduto, Santillo et Killings, 1998; Kohl, Lengua et McMahon, 2000 ; Marcon, 1999; Pelco, Jacobson, Ries et Melka, 2000; Zellman et Waterman, 1998). De plus, la qualité des contacts entre l'école et les parents, un aspect absent des deux typologies décrites précédemment, l'emporterait sur la quantité d'échanges et serait l'élément qui aurait le plus d'effets positifs chez les enfants. À l'inverse, la fréquence des contacts serait généralement associée aux difficultés encourues par l'enfant (Kohl, Lengua et McMahon, 2000; Marcon, 1999; Reynolds, Weissberg et Kasprow, 1992) puisque ce serait lors d'événements fâcheux que l'école aurait tendance à communiquer avec les parents (Deslandes, 2001; Marcon, 1999). Par contre, les parents amorceraient davantage de contacts avec le milieu scolaire lorsque leur enfant irait bien ou serait talentueux (Marcon, 1999). Dans ces circonstances, la collaboration avec l'école serait généralement initiée par les parents (Griffith, 1998). Par ailleurs, la collaboration entre les enseignants et les parents aurait facilement tendance à s'envenimer lors de contacts au sujet des comportements violents d'un enfant (Peña, 2000). Cette situation générerait un besoin accru de soutien social chez ces parents (Reynolds, Weissberg et Kasprow, 1992).

\subsection{LES COMPORTEMENTS VIOLENTS AU PRÉSCOLAIRE ET AU PRIMAIRE}

Depuis quelques années, les chercheurs accordent une attention particulière à la prévention et à l'intervention précoces des comportements violents en milieu scolaire (Algozzine et Kay, 2002; Evans, Okifuji, Engler et Bromley, 1993 ; Reid, Eddy, Fetrow et Stoolmiller, 1999; Reynolds, Weissberg et Kasprow, 1992; Sheridan, Kratochwill et Elliott, 1990; Webster-Stratton, Reid et Hammond, 2001; Zellman et Waterman, 1998). Selon eux, consolider la collaboration entre l'école et les parents s'avère une priorité afin de contrer les comportements violents au préscolaire et au primaire. En cette matière, la collaboration entre l'école et les parents apparaît comme un des aspects les plus importants des programmes de prévention et d'intervention précoces (Bélanger, Bowen et Rondeau, 1999; Bowen, Desbiens, Rondeau 
et Ouimet, 2000a; Turcotte, Lamonde et Lindsay, 2002). Un élément fondamental de ces programmes repose sur des pratiques éducatives parentales favorisant l'encadrement positif des enfants à la maison (Royer, Morand, Desbiens, Moisan et Bitaudeau, 2000). Cet encadrement consiste à limiter les interventions coercitives, à renforcer les encouragements, à développer une plus grande constance dans les pratiques éducatives des parents, ainsi qu'une plus grande cohérence avec celles de l'école (Armstrong, Caron et Guimond, 1992; Cavell, 2000 ; Dangel et Polster, 1984). L'évaluation de tels programmes s'avère toutefois récente (Centre de recherche et d'intervention sur la réussite scolaire, 2002). La poursuite des recherches en ce domaine devrait éventuellement permettre de valider de tels constats.

Par ailleurs, Grolnick, Benjet, Kurowski et Apostoleris (1997) relèvent que les parents ont tendance à moins s'impliquer dans la vie scolaire de leur enfant ayant des comportements violents, considérant désagréables les interactions avec l'école et préférant ainsi ignorer la situation-problème. Dans le même sens, Deslandes (2001, p. 265) constate que «les parents sont davantage portés à aider un enfant qui, pour la première fois, fait preuve de violence à l'école». Ces derniers constats appuient l'idée de l'importance de la prévention précoce afin d'éviter l'émergence de tels comportements.

Bref, s'il y a tout lieu que les parents collaborent très tôt avec l'école, il est également important de se demander à partir de quel moment l'institution scolaire s'est montrée ouverte à cette pratique. Pour donner une meilleure idée de l'évolution historique du concept de collaboration entre l'école et les parents, nous présentons diverses perspectives dans le temps.

\subsection{LES DIVERSES PERSPECTIVES DANS LE TEMPS}

La collaboration entre l'école et les parents n'est pas nouvelle. Du début de la colonisation française en Amérique jusqu'à la fin des années 1960, l'institution scolaire faisait partie intégrante de la communauté en étant perçue comme un prolongement de la famille (Gareau et Sawatsky, 1995). Au cours de toutes ces années, l'autorité des enseignants, en majorité des ecclésiastiques, se substituait même à celle des parents. Les religieux n'admettaient pas que ceux-ci interviennent dans la vie scolaire de leur enfant, considérant leur présence à l'école comme une entrave à la discipline scolaire.

Puis, au cours de la décennie 1970, avec le début de la professionnalisation de l'enseignement (Dubet, 1997), le métier d'enseignant se complexifie. Il apparaît dorénavant comme une force de connaissances 
(Pourtois et Desmet, 1997) et de notoriété (Montandon, 1994). Ce phénomène est accru par le passage des programmes de formation de l'école normale à l'université (Gareau et Sawatsky, 1995). De plus, les administrations scolaires exercent un leadership qui contribue à maintenir l'absence de pouvoir aux parents (Berger Hepworth, 1991). «Les professionnels de l'éducation ont longtemps considéré que les parents n'ont aucune autorité en matière d'enseignement» (Montandon, 1994, p. 189). De plus, on note toujours une conception largement répandue à savoir que la participation parentale s'avère possible, mais essentiellement lorsqu' elle fait suite aux demandes formulées par l'école (Zellman et Waterman, 1998).

Puis, depuis le début des années 1980, un effort de rapprochement entre l'école et les parents s'amorce (Berger Hepworth, 1991). Les notions de collaboration ou de partenariat entre les deux milieux prennent un essor (Montandon et Perrenoud, 1994; Pourtois et Desmet, 1997), tant et si bien qu'au début des années 1990, soit au moment où les résultats de plusieurs recherches dans le domaine de l'éducation invitent à une plus grande présence des parents à l'école, on observe un changement de cap. Les parents, beaucoup plus instruits, s'intéressent dorénavant au processus de scolarisation et de socialisation de leur enfant (Conseil de la famille et de l'enfance, 2000 ; Pourtois et Desmet, 1997). Or, ce niveau d'instruction et cet intérêt accru peuvent parfois devenir une source d'incompréhension, voire de conflits, entre l'école et les parents. Cette situation est davantage exacerbée lorsque ces derniers surveillent de près les résultats de leur enfant (de Singly, 2000; Dubet, 1997; Dubet et Martuccelli, 1996; Van Zanten et Duru-Bellat, 1999; Peña, 2000 ; Pourtois et Desmet, 1997). Perrenoud (2001a, 2001b) avance même que les effets de la nouvelle économie interfèrent de plus en plus entre les milieux scolaire et familial, en créant davantage de pression entre les deux.

Par ailleurs, selon Christenson et Sheridan (2001), l'institution scolaire réalise actuellement un autre passage: elle ne se questionne plus uniquement sur les modalités de participation des parents, mais également sur le soutien à leur apporter en vue de leur permettre de contribuer davantage au développement socioscolaire de leur enfant. Il ne s'agit plus seulement de prévenir l'échec et l'abandon scolaires, mais d'établir à l'école un milieu de résilience (Reynolds, 1999). En ce sens, un milieu est résilient lorsqu'il vise à outiller les enfants afin qu'ils apprennent à surmonter les difficultés ou les obstacles qu'ils rencontrent dans leur processus de scolarisation et de socialisation (Ehrensaft et Tousignant, 2004).

À cet égard, Epstein (1992) propose quatre modèles qui tracent l'évolution historique de l'influence entre l'école et les parents. D'un système fermé et favorisant une relation séparée, elle passe à un système plus écologique (Bronfenbrenner, 1986; Phillips-Smith, Connell, Wright, Sizer, Norman, 
Hurley et Walker, 1997) qui permet tant aux enseignants qu'aux parents de s'influencer mutuellement en devenant de véritables partenaires dans l'éducation des enfants. Les quatre modèles de l'influence entre l'école et les parents d'après Epstein (1992) sont présentés au tableau 2.3.

\section{TABLEAU 2.3}

\section{Les quatre modèles de l'influence entre l'école et les parents d'après Epstein (1992)}

1) Distincte (separate).

Met l'accent sur les contributions séparées des institutions scolaires et familiales. Les enseignants et les parents agissent indépendamment les uns des autres ou dans deux mondes en parallèle. L'école ne communique avec les parents que lors de problèmes graves.

2) Séquentielle (sequenced). Montre le développement des responsabilités selon des stades, notamment celles des parents, qui sont surtout responsables de l'éducation de l'enfant d'âge préscolaire. Par la suite, l'école assume de plus en plus cette charge.

3) Intégrée (embedded). Témoigne de l'interaction constante entre les différentes composantes du système social (individus, groupes, organismes) et de leur influence réciproque.

4) Partagée (overlapping). Reconnaît le lien et la dynamique existant non seulement entre l'institution scolaire et la famille, mais également en interaction avec la communauté. Ce partage renforce l'implication parentale dans la vie scolaire de l'enfant.

Le dernier modèle d'Epstein (1992), soit celui de l'influence partagée entre l'école, la famille et la communauté, a donné lieu à l'élaboration de la typologie de l'implication parentale (Epstein, 1995), telle que décrite au point 1.2. du présent ouvrage. C'est également celui qui retient notre attention en raison de son ouverture favorisant des formes reconnues d'implication parentale dans la vie scolaire de l'enfant. Le modèle de l'influence partagée entre l'école, la famille et la communauté (Epstein, 1995) est illustré à la figure 2.1.

Selon le dernier modèle d'Epstein, les trois milieux que sont l'école, la famille et la communauté peuvent se rapprocher ou s'éloigner en fonction des forces qu'ils exercent les uns sur les autres, soit les caractéristiques, les philosophies et les pratiques propres à chacun d'eux. En ce sens, la question des comportements violents au préscolaire et au primaire peut s'étudier selon de multiples combinaisons, soit en fonction d'un seul milieu, 
soit en interaction avec un, deux ou trois autres d'entre eux. En ce qui a trait aux enfants de 3 à 9 ans, les deux milieux les plus dynamiques de ce système en interaction sont la famille, qui représente le milieu immédiat de l'enfant, et l'école, qui est l'un des premiers milieux institutionnels avec lesquels il entre en contact.

FIGURE 2.1

Le modèle de l'influence partagée entre l'école, la famille et la communauté d'après Epstein (1995)

Tiré de Deslandes (2001, p. 263),

avec la permission des Publications du Québec

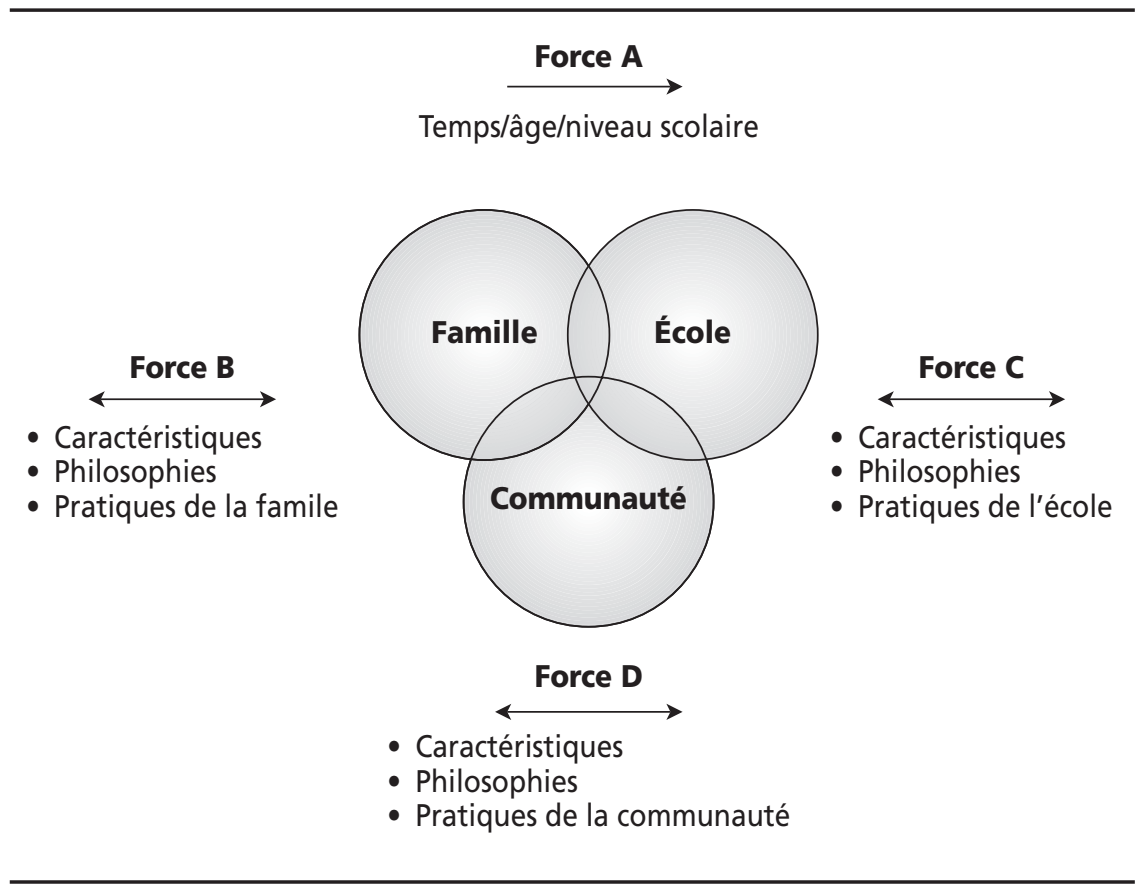

\subsection{LES NOUVELLES RÉALITÉS FAMILIALES}

Pour mieux saisir la teneur du parcours historique de la collaboration entre l'école et les parents, il importe de mettre en lumière des changements importants qui sont survenus au niveau de la famille au cours des trente dernières années (Conseil de la famille et de l'enfance, 2000). Ces changements, en plus de diversifier les réalités familiales, deviennent des éléments de contextualisation permettant de mieux comprendre les nouvelles responsabilités que les parents exercent à l'égard du milieu scolaire. Parmi ces 
nouvelles réalités se trouvent la centration sur l'enfant, l'entrée massive des mères sur le marché du travail, la diversité des structures familiales et le conflit travail-famille.

Concernant la centration sur l'enfant, l'Institut Vanier (2000) observe qu'elle est occasionnée par une baisse de la fécondité, due à une conjugaison de réalités individuelles et de facteurs économiques. De cette baisse de fécondité résulte une réduction du nombre d'enfants par famille et une proportion accrue d'enfants uniques (Conseil de la famille et de l'enfance, 2000). Ces petites familles deviennent plus centrées sur les enfants. Cette centration, soit cet intérêt accru pour l'enfant, amène les parents à surveiller son cheminement de près, à exercer des pressions en faveur de sa réussite scolaire (Van Zanten et Duru-Bellat, 1999) et, même, à demander au milieu scolaire de rendre des comptes (Perrenoud, 2001a, 2001b).

Par ailleurs, l'entrée massive des mères sur le marché du travail fait qu'elles disposent de moins de temps pour entretenir des contacts avec l'école (Deslandes, 2001; Organisation de coopération et de développement économiques, 1997). Actuellement, trois mères sur quatre ayant un enfant d'âge scolaire et $70 \%$ de celles ayant un enfant de trois à cinq ans occupent un emploi (Institut Vanier, 2000). Il s'agit en 25 ans d'une augmentation de la présence sur le marché du travail qui correspond à $20 \%$ des mères d'enfants âgés de six à 15 ans et à $40 \%$ de celles d'enfants de moins de six ans (Higgins et Duxbury, 2002).

Concernant la diversité des structures familiales, de 1971 à 1996, la proportion de familles intactes a chuté de $87 \%$ à $78 \%$. À l'inverse, la proportion de familles monoparentales est passée de $14 \%$ à $22 \%$. Ces dernières ont généralement beaucoup de difficulté à concilier le travail et la vie familiale. L'une de ces difficultés est le stress accru par le manque de ressources financières (Higgins et Duxbury, 2002). Il découle de cette situation que les mères de familles monoparentales s'impliquent moins directement à l'école que les parents de familles intactes. Il leur faudrait des horaires de rencontres plus souples pour pouvoir être davantage présentes à l'école à l'extérieur des heures de travail (Grolnick, Benjet, Kurowski et Apostoleris, 1997). Par ailleurs, les mères de familles monoparentales sont tout autant impliquées à la maison auprès de leurs enfants que celles des familles intactes. En raison d'un manque chronique de temps et d'argent, elles donnent toutefois la priorité aux contacts avec l'enseignant, au détriment de la participation à des activités scolaires (Kohl, Lengua et McMahon, 2000).

Le conflit travail-famille ne concerne pas que les familles monoparentales puisqu'il s'étend aux familles biparentales et biactives (Higgins et Duxbury, 2002). On observe en effet un accroissement des heures de 
travail chez tous les segments de la population active. Cet accroissement se traduit par un minimum de 45 heures par semaine qu'une majorité de Canadiens consacrent dorénavant à leur emploi (Higgins et Duxbury, 2002). L'augmentation de l'activité professionnelle engendre une présence moindre à la maison. Par conséquent, il y a une diminution du temps consacré à l'enfant (Conseil de la famille et de l'enfance, 2000); ce qui n'est pas sans occasionner des pressions supplémentaires sur les parents. Leur manque de temps entrave leurs interactions avec l'école (Marcon, 1999), autant que celles qu'ils entretenaient auparavant avec leur enfant à la maison (Higgins et Duxbury, 2002).

\subsection{LES FACTEURS DE SOUTIEN ET LES OBSTACLES SOUS-JACENTS À LA COLLABORATION}

Selon Epstein (1995), il n'y a pas que les nouvelles réalités familiales qui nuisent à la collaboration entre l'école et les parents. Les quatre constats qui ressortent le plus clairement de ses recherches sont que la collaboration entre l'école et les parents:

1) diminue avec les années de scolarité de l'enfant, à moins qu'un travail ne soit effectué par l'école pour maintenir une telle pratique;

2) est davantage présente chez les parents issus de milieux aisés, à moins que les écoles travaillent à rejoindre les familles vivant en contexte de pauvreté;

3) provient davantage des écoles de milieux défavorisés qui communiquent avec les parents essentiellement lorsqu'il y a des problèmes, à moins qu'elles instaurent un équilibre avec des contacts plus positifs;

4) touche moins les familles monoparentales, les parents qui travaillent, ceux qui vivent loin de l'école et les pères qui disposent de moins de temps pour s'impliquer, à moins que les écoles innovent en permettant à ces familles de faire du bénévolat à différents moments de la journée.

Ces constats demandent toutefois un approfondissement en raison de certains facteurs qui fournissent un soutien ou qui deviennent des obstacles sous-jacents à la collaboration entre l'école et les parents. L'âge des enfants, le sentiment de compétence éducative parentale et certaines variables, telles que le milieu socioéconomique et l'appartenance ethnoculturelle des parents, sont les principaux facteurs à considérer. 


\subsubsection{L'âge des enfants}

L'âge des enfants est un facteur déterminant qui influence la collaboration entre l'école et les parents, car cette collaboration est plus grande lorsqu'ils sont au début de leur processus de scolarisation et de socialisation (Eccles et Harold, 1991; Montandon, 1996; Organisation de coopération et de développement économiques, 1997; Reynolds, Weissberg et Kasprow, 1992). Par ailleurs, le processus d'autonomie qui s'installe ainsi que la taille et la bureaucratisation des écoles secondaires contribuent progressivement à l'éloignement des parents (Deslandes, 2001). Le sentiment de compétence éducative limitée et d'étrangeté qu'ils ressentent par rapport au milieu scolaire et à ses programmes ne peut qu'accroître cette distance (Larose, Bédard et Terrisse, 2002). La participation parentale, définie cette fois en termes d'aspirations élevées et d'une bonne communication entre les parents et les adolescents, aurait toutefois des effets positifs sur la réussite scolaire des élèves du secondaire (Keith, Keith, Quirk, Sperduto, Santillo et Killings, 1998).

\subsubsection{Le sentiment de compétence éducative des parents}

Le sentiment de compétence éducative des parents se révèle un facteur de soutien sous-jacent à la collaboration avec le milieu scolaire (Eccles et Harold, 1993). S'ils se sentent compétents dans l'éducation de leur enfant, les parents feront davantage d'appels téléphoniques à l'école, plus de bénévolat, et ils participeront davantage aux activités scolaires (HooverDempsey, Bassler et Brissie, 1992). Le sentiment de compétence éducative parentale, dans la foulée des travaux d'Ozer et Bandura (1990), se définit comme étant «la croyance qu'a un parent d'exercer une influence positive sur son enfant» (Hoover-Dempsey, Bassler et Brissie, 1992, p. 287). Cette expression se définit également comme «la croyance que quelqu'un est capable d'atteindre les résultats désirés à partir des efforts qu'il déploie et de ceux que déploient les autres pour lui » (Hoover-Dempsey, Bassler et Brissie, 1992, p. 291). En ce sens, Grolnick, Benjet, Kurowski et Apostoleris (1997) soutiennent que les parents s'impliquent davantage lorsqu'ils se perçoivent efficaces et qu'ils se sentent de bons éducateurs. De plus, les parents auront tendance à participer davantage s'ils ont développé ce sentiment de compétence éducative ou cette conviction d'exercer une telle influence chez leur enfant (Hoover-Dempsey et Sandler, 1995, cité dans Deslandes, 1999). 


\subsubsection{Les parents vivant en contexte de pauvreté}

Le sentiment de compétence éducative limitée et d'étrangeté s'avère plus fréquent chez les parents vivant en contexte de pauvreté (Kohl, Lengua et McMahon, 2000 ; Larose, Bédard et Terrisse, 2002). En ce sens, le milieu socioéconomique se révèle un important facteur prédicteur de la participation parentale à l'école (Dubet et Martuccelli, 1996; Lareau, 1987; Lytton et Pyryt, 1998; Marcon, 1999 ; Pelco, Jacobson, Ries et Melka, 2000 ; Raffaele et Knoff, 1999). En effet, les parents ayant une plus faible scolarité et vivant en contexte de pauvreté ne pourraient ou ne voudraient pas s'impliquer dans la scolarisation de leur enfant, car ils se sentiraient facilement surpassés (Epstein, 1992; Kohl, Lengua et McMahon, 2000). Ces parents se sentiraient également peu ou pas compétents à aider celui-ci au plan scolaire en raison de leur propre passé parsemé d'échecs. Cette situation occasionnerait un transfert de la compétence éducative aux enseignants, mieux éduqués et provenant des classes moyenne et moyenne-supérieure, qui pourraient percevoir ces parents selon un cadre hiérarchique de références (Kohl, Lengua et McMahon, 2000 ; Marcon, 1999).

Par ailleurs, les parents vivant en contexte de pauvreté ont leur propre forme d'implication auprès de leur enfant à la maison. Celle-ci compense leur manque de présence à l'école (Epstein, 1992). Malgré les différences d'apport entre ces parents et ceux des classes moyenne et moyennesupérieure, leur forme d'implication bénéficierait tout autant à leur enfant (McCarthey, 2000). Il ne s'agirait ni d'un manque (Cloutier, Champoux et Jacques, 1997) ni d'une démission (Lahire, 1995) face à l'école, mais d'une conception qui confère à l'institution scolaire l'entière responsabilité d'agir auprès de l'enfant. Cette action est même perçue par certains parents comme un tremplin permettant à leur enfant d'accéder plus tard à de meilleures conditions de vie que les leurs (Glassman, 1994).

Ainsi, vivre en contexte de pauvreté dans un quartier aux ressources limitées signifie pour ces familles qu'elles doivent affronter des stress importants, et ce, de façon quotidienne (Lareau et Horvat, 1999; Raffaele et Knoff, 1999). Un tel contexte de vie les oblige à mettre en œuvre des moyens et des stratégies permettant de s'adapter, voire de survivre (Eccles et Harold, 1993). Pour ces parents, «leurs sentiments de subir ou, à l'inverse, de contrôler les événements de leur vie a pour effet de susciter soit un intérêt, soit un désintérêt pour les apprentissages scolaires, de même que développer une attitude soit positive, soit négative par rapport à l'école » (Comeau et Salomon, 1994, p. 206). Par conséquent, pour agir auprès d'eux, il faut des plans d'accès, des mesures compensatoires, des passerelles qui assurent leur implication accrue à l'école, voire une collaboration de tous les instants entre ces deux milieux (Algozzine et Kay, 2002; Raffaele et Knoff, 1999). 
De telles barrières entre l'école et les parents vivant en contexte de pauvreté requièrent des prises en considération spéciales pour qu'on puisse les rejoindre dans leur quotidien (Eccles et Harold, 1993). Par contre, les écoles qui offrent un soutien matériel et psychologique aux enfants, ainsi que des ateliers de développement des compétences sociales, peuvent compenser certaines limites encourues par l'absence des parents vivant dans un tel contexte. Il peut également s'agir d'ateliers de formation sur les pratiques éducatives parentales ou de groupes de parentage. Ces ateliers sont un moyen plus facile de cibler certains parents afin de les inciter à s'impliquer dans la vie scolaire de leur enfant. Selon Zellman et Waterman (1998), les parents qui s'impliquent à la suite d'une telle formation diminuent l'utilisation de certaines pratiques éducatives négatives, telles que celles qui sont parfois présentes dans des milieux familiaux aux prises avec une problématique de négligence. Par ailleurs, devant de telles mesures incitatives, certains parents peuvent poser des gestes de résistance (Van Zanten, 2001) ou de survie (Vatz Laaroussi, 1996) afin de préserver leur identité et de se protéger (Dubet et Martuccelli, 1996). Paradoxalement, ces mesures peuvent contribuer à accroître leur distance avec l'école.

\subsubsection{Les parents des classes moyenne et moyenne-supérieure}

À l'inverse, les contacts entre l'école et les parents regroupés dans les classes moyenne et moyenne-supérieure sont plus fréquents en raison d'un niveau d'instruction et de ressources financières plus élevés (Montandon, 1996). Ces parents sont davantage impliqués dans l'éducation de leurs enfants, qui réussissent mieux à l'école (Epstein, 1992). Ils seraient également davantage conscients de l'importance de soutenir directement leur enfant dans leur processus de scolarisation et de socialisation. Ils valoriseraient ainsi l'école aux yeux de leur enfant afin qu'elle agisse comme un tremplin social (Pourtois et Desmet, 1997). De surcroît, «en essayant de répertorier en positif ce que serait un bon parent d'élève, on décrit une personne qui entretient des contacts avec les enseignants, qui s'intéresse au travail scolaire [...]. Il s'agit en fait du profil d'un parent d'une nouvelle classe moyenne et moyenne-supérieure, consommateur averti et usager responsable, aux attentes bien définies» (Montandon, 1996, p. 71).

Également, les parents des classes moyenne et moyenne-supérieure se sentiraient aisément coupables et responsables de ce qui arrive à leur enfant (Dubet, 1997; Dubet et Martuccelli, 1996). Une attitude de consommateurs de services se serait développée chez eux (Hirschhorn, 2001; de Singly, 2000; Dubet, 1997; Dubet et Martuccelli, 1996; Van Zanten et Duru-Bellat, 1999; Pourtois et Desmet, 1997; Vincent et Tomlinson, 1997). Le consumérisme donne place à une connaissance des rouages administratifs, à une implication active et à un empowerment accru de ces parents. 
Cette situation apparaît alors comme une «appropriation progressive de l'école» de leur part (Conseil de la famille et de l'enfance, 2000, p. 7), et ce, de façon individuelle ou collective. Toutefois, le consumérisme est une source potentielle de conflits et d'évaluation du monde scolaire, en entravant la collaboration (Perrenoud, 2001, 2002). L'implication parentale dans la vie scolaire de l'enfant et la collaboration entre l'école et les parents deviendraient ainsi de nouvelles expressions pour désigner certaines valeurs davantage propres aux classes moyenne et moyenne-supérieure (Johnson, 1994).

\subsubsection{L'origine ethnoculturelle des parents}

L'origine ethnoculturelle des parents apparaît comme un facteur critique limitant leur implication à l'école (Desimone, 1999; Eccles et Harold, 1993; Kohl, Lengua et McMahon, 2000; Lareau et Horvat, 1999; Lee Yao, 1993 ; Peña, 2000 ; Rich, 1993). À cet effet, les difficultés socioéconomiques et l'ethnicité coïncident souvent avec le manque de présence parentale à l'école (McAndrew, 2002). Selon Peña (2000), l'accès aux parents vivant en contexte minoritaire pluriethnique est difficile parce qu'ils se sentent intimidés par le personnel et la structure scolaires. Des différences culturelles et des cadres de référence autres expliqueraient la distance qui se crée entre l'école et ces familles. De plus, des difficultés de communication, surtout lorsque ces parents ne maîtrisent pas suffisamment la langue d'usage, seraient souvent à l'origine de ces difficultés (Lee Yao, 1993).

Selon McAndrew (2002), le facteur ethnoculturel exige l'adoption de mesures spécifiques afin de favoriser le développement de liens entre l'école et les familles vivant en contexte minoritaire pluriethnique. Développer de tels liens implique l'utilisation de moyens et de stratégies favorisant l'implication de ces parents à l'école, en vue d'établir une collaboration entre ces deux milieux (Rich, 1993). Par exemple, Quintero (1999) met en valeur le caractère informel que doit garder l'environnement, de même que les contacts offrant la possibilité de mettre l'ethnicité en valeur. Peña (2000) insiste pour que les difficultés quotidiennes vécues par ces familles soient prises en considération en tant que barrières limitant l'accès à l'école. Le fait de prendre ces difficultés en considération est d'autant plus important que l'école les perçoit comme des interférences dans l'implication des parents.

Concernant la situation des enfants et des parents des groupes ethnoculturels, Zellman et Waterman (1998) mettent en lumière des constats intéressants. Notamment, lors d'une analyse transversale, il appert que les parents vivant en contexte minoritaire pluriethnique d'une même catégorie socioéconomique coopéreraient davantage à la vie scolaire de leurs 
enfants que les autres parents, y compris ceux ne vivant pas dans un tel contexte. Dans le même ordre d'idées, il appert également que ce seraient les parents des communautés afro-américaines qui s'impliqueraient le plus activement à l'école que fréquente leur enfant. En ce sens, les enseignants de ces écoles, aussi membres de ces groupes, les inciteraient davantage à la participation.

Par ailleurs, la majorité des parents d'origine asiatique œuvreraient davantage sur le plan des devoirs et des activités parascolaires, en ayant un grand respect pour l'autorité de l'enseignant et en lui faisant confiance. Ces derniers communiqueraient peu avec l'école, ce geste étant considéré comme de la surveillance (Lee Yao, 1993).

Finalement, la participation des parents autochtones et les aspirations élevées qu'ils entretiennent envers la réussite scolaire au secondaire auraient des effets bénéfiques chez leur adolescent (Keith, Keith, Quirk, Sperduto, Santillo et Killings, 1998). À ce sujet, Kohl, Lengua et McMahon (2000) ne perçoivent pas de différences statistiquement significatives entre la participation des parents d'enfants caucasiens de la maternelle et les parents afro-américains. Or, à l'inverse, lors d'analyses de parcours individuels, des parents des communautés afro-américaines ressentiraient une plus grande distance par rapport à l'école. Les raisons évoquées par ces chercheurs pour justifier cette distance consistent en de mauvaises expériences scolaires passées ou une représentation de leur sentiment de compétence éducative parentale, définie précédemment par HooverDempsey, Bassler et Brissie (1992), qui n'inclut pas la composante de la collaboration avec l'école.

Pour toutes ces raisons, Desimone (1999) soutient l'idée de l'importance de poursuivre la recherche afin de mieux comprendre les enjeux liés aux diversités de pratiques d'implication chez les parents vivant en contexte minoritaire pluriethnique.

\subsection{CE QU'EN DISENT LES ÉTUDES SUR LE MILIEU SCOLAIRE}

Les études sur la collaboration entre l'école et les parents précédemment répertoriées se centrent majoritairement sur ses bienfaits, notamment chez l'élève. Elles répertorient également les facteurs de soutien et les obstacles sous-jacents à cette collaboration, en fonction des variables sociodémographiques des parents d'enfants ayant des comportements violents envers leurs pairs au préscolaire et au primaire. 
Par ailleurs, Christenson et Sheridan (2001) soutiennent qu'il y a toujours une relation entre l'école et les parents. Ceux-ci sont soit actifs, soit passifs. Dans ce dernier cas, ils deviendront actifs lors de difficultés. Selon Epstein (citée par Christenson et Sheridan, 2001, p. 24), «20\% des parents sont des partenaires actifs; $70 \%$ le seraient si l'école leur fournissait plus d'informations ou de soutien; seulement $10 \%$ d'entre eux ne collaborent pas, ne le feront pas et ne peuvent pas collaborer, en raison de difficultés personnelles majeures». Les pratiques des écoles et des enseignants semblent donc être l'élément le plus déterminant en faveur de la collaboration avec les parents (Adams et Christenson, 2000; Deslandes, 2001, 2002b; Dubet et Martuccelli, 1996; Eccles et Harold, 1993; Epstein, 2001; Grolnick, Benjet, Kurowski et Apostoleris, 1997; Martin, Tett et Kay, 1999; Organisation de coopération et de développement économiques, 1997; Saint-Laurent, Royer, Hébert et Tardif, 1994; Salomon et Comeau, 1998).

Si les parents se sentent les bienvenus à l'école, il y a davantage de chances qu'ils participent, s'impliquent et collaborent. Toutefois, les possibilités d'action laissées aux parents découlent essentiellement des perceptions et des décisions prises par le milieu scolaire (Saint-Laurent, Royer, Hébert et Tardif, 1994). Tout le travail déployé en faveur de l'implication parentale dans la vie scolaire de l'enfant n'agirait donc qu'en faveur du bénévolat, de la participation aux activités parascolaires et de la communication, soit des échanges entre les deux milieux au sujet des progrès scolaires de l'enfant (Feuerstein, 2000), ce constat prévalant surtout pour les élèves à haut risque d'échec et d'abandon scolaires (Gavin et Greenfield, 1998; Potvin, Deslandes, Beaulieu, Marcotte, Fortin, Royer et Leclerc, 1999; Royer, Saint-Laurent, Bitaudeau et Moisan, 1995).

D'autres auteurs ont tenté de cerner les obstacles sous-jacents à la collaboration ou à la relation entre l'école et les parents. Il s'agit notamment du manque de clarté dans la communication, évoqué par Christenson et Sheridan (2001), des malentendus cités par Dubet (1997), des incompréhensions mutuelles observées par Montandon (1994), du transfert des responsabilités concernant l'éducation des enfants et justifiant leurs problèmes, constaté par Lazar et Slostad (1999) et, finalement, de l'échec de la communication, voire des revendications mutuelles, reconnu par Claes et Comeau (1996).

L'ampleur des désaccords entre l'école et les parents semble augmenter en fonction de l'ampleur des difficultés encourues par l'enfant (Christenson et Sheridan, 2001; Lazar et Slostad, 1999). Plus les difficultés sont importantes, selon Dubet et Martuccelli (1996), plus les parents ont peur d'être confrontés par le personnel scolaire. De leur côté, les enseignants ont peur d'être touchés dans leur identité professionnelle et de faire remettre en 
question leurs pratiques. Or, la situation-problème de l'enfant n'a guère de chances de s'améliorer si tant l'école que les parents ne s'efforcent pas réellement de collaborer. "C'est ce que signifie l'appel constant à la communication entre l'école et les parents» (Dubet et Martuccelli, 1996, p. 111).

Quoi qu'il en soit, un point central de malentendu entre le milieu scolaire et familial demeure l'intérêt que les parents éprouvent face à la scolarisation et la socialisation de leur enfant aux yeux de l'enseignant. Sont-ils impliqués et veulent-ils s'impliquer? À ce sujet, les enseignants entretiennent, dans l'ensemble, une image négative du rôle des parents (Montandon, 1994) et de leur intérêt, voyant en eux des démissionnaires (Martichoux, 2000). À l'inverse, les parents affirment qu'ils s'engagent à la maison selon leurs propres pratiques et, pour s'acquitter adéquatement de ce rôle, qu'ils ont besoin d'informations de la part de l'école (Epstein et Dauber, 1991; Epstein, 2001).

En guise d'exemples illustrant cette divergence dans la perception de la collaboration entre l'école et les parents, Salomon et Comeau (1998) ont étudié un échantillon de près de 4400 sujets (2 897 parents, 181 directions d'école et 1312 enseignants du primaire). En lien avec les six formes d'implication énumérées dans la typologie d'Epstein (1995), ces chercheures ont remarqué que les enseignants doutent des bénéfices de la participation parentale. Â ce sujet, un tiers d'entre eux ne voient pas ce que les parents viennent faire à l'école, et la moitié ne pensent pas que leur présence améliore la qualité des activités scolaires ou les stimule pédagogiquement. Par ailleurs, elles ont également remarqué que les parents sont en général systématiquement plus convaincus que les enseignants des bienfaits de leur participation. Quant aux parents non-membres des comités d'école, ils semblent s'intéresser davantage à une participation ayant trait à leur propre enfant qu'à des modifications majeures du milieu éducatif, et une minorité d'entre eux considèrent que la participation à l'école n'est qu'une mode. Les parents qui sont membres des comités d'école, eux, préconisent qu'on leur confie un rôle consultatif plus important.

À ce sujet, Saint-Laurent, Royer, Hébert et Tardif (1994) constatent des différences de perceptions importantes chez un échantillon de plus de 1500 sujets (957 parents et 630 enseignants du primaire). Les parents disent investir passablement de temps et d'énergie auprès de leur enfant et vouloir collaborer avec l'école. De plus, ils se déclarent satisfaits des informations reçues et de la communication entretenue avec l'école. Par contre, les parents provenant de groupes socioéconomiquement défavorisés désirent obtenir davantage de renseignements sur les programmes et le matériel scolaires. Du côté des enseignants, ceux-ci surestiment le degré de satisfaction des parents quant aux renseignements qui leur sont transmis et 
voient peu la nécessité de tenir des réunions d'information. Finalement, ils désirent limiter leur disponibilité à l'égard des activités parascolaires étant donné leur manque de temps, même s'ils croient à la collaboration.

Par ailleurs, avec un échantillon de 700 sujets (558 parents et 142 enseignants surtout du primaire), Gettinger et Waters-Guetschow (1998) avancent des conclusions allant dans le même sens que celles de Salomon et Comeau (1998) ou de Saint-Laurent, Royer, Hébert et Tardif (1994). Ces enseignants considèrent en effet que les parents ont plus de barrières et moins d'occasions de participer aux activités tenues à l'école que les parents ne le perçoivent eux-mêmes. Par ailleurs, certains parents notent un manque d'occasions de s'engager à l'école, une indifférence de la part du personnel scolaire et l'aspect intimidant du langage particulier propre à ce milieu (HooverDempsey, Bassler et Brissie, cités dans Peña, 2000). De plus, s'ils s'impliquent trop fréquemment ou de façon trop intrusive, les parents sentent les effets pervers de la distance (Reynolds, Weissberg et Kasprow, 1992).

Quant à Montandon (1994), qui analyse l'identité professionnelle des enseignants sous l'angle des obstacles sous-jacents à la collaboration, elle croit tout comme Cifali (1997) qu'elle est actuellement remise en question. Parallèlement à ce constat, Gohier (1997) s'interroge sur la professionnalisation des enseignants en fonction des nouveaux rôles éducatifs que ce mouvement leur impose. Dans le même sens, Perrenoud (2001a) observe que, dans un tel contexte de remise en question, les enseignants se sentent menacés et dévalorisés. Bref, il n'est pas étonnant de constater une plus grande réticence de leur part à l'égard des parents, puisqu'ils ont peur d'être envahis, d'être exposés à de fréquentes critiques, d'affronter des attentes inconciliables et de vivre avec davantage de conflits (Pourtois et Desmet, 1997). À ce sujet, Montandon (1994) note un paradoxe entre le désir des enseignants d'être considérés comme des professionnels et la difficulté qu'ils ont à assumer les nouvelles responsabilités qui leur sont dévolues, soit de consentir une plus grande place aux parents dans le processus de prise de décisions et de faire preuve d'une plus grande transparence en regard du vécu quotidien de l'enfant, notamment au préscolaire et au primaire.

Cullingford et Morrison (1999) avancent que les enseignants se plaignent aussi d'un manque de temps et d'un surcroît de tâches. En ce sens, la collaboration avec les parents représente pour eux un surplus d'efforts en plus de se révéler parfois une expérience difficile. Ces auteurs précisent que la racine du problème ne repose pas sur les personnes ou sur la personnalité de ces dernières, mais plutôt sur les circonstances qui nuisent à un rapprochement entre les milieux scolaire et familial. À cet égard, tout en valorisant la collaboration, les enseignants appréhendent l'ingérence des parents, ce qui toucherait à leur identité professionnelle. 
En définitive, la collaboration entre l'école et les parents semble facile à proposer mais difficile à mettre en place (Conseil de développement de la recherche sur la famille du Québec, 2002; Montandon, 1996; Montandon et Perrenoud, 1994; Pourtois et Desmet, 1997). En ce sens, Deslandes (1999) considère le partenariat comme un projet en devenir. Quant à l'implication parentale, telle que définie par Epstein (1995), elle comporte des bienfaits dont les enfants tirent grandement avantage. Toutefois, les résultats d'études qui visent à stimuler la participation parentale relèvent peu de différences significatives sur l'augmentation des résultats scolaires des élèves. Cette situation semble attribuable au fait que les parents qui présentent un plus grand intérêt à l'égard du processus de scolarisation et de socialisation sont ceux dont les enfants réussissent le mieux et sont le mieux adaptés à l'école (Deslandes, 2001) et, en bout de ligne, qui collaborent le plus avec le personnel scolaire.

\section{UN COURT BILAN DES DIFFICULTÉS DE COLLABORATION}

Cette troisième partie présente un court bilan des difficultés inhérentes à l'établissement d'un travail de collaboration entre l'école et les parents. Beaucoup d'avantages découlent de la collaboration entre les milieux scolaire et familial, et ce, en faveur de l'enfant et de son développement. Il est toutefois plus réaliste de considérer que cette collaboration concerne un enfant provenant des classes moyenne et moyenne-supérieure. De plus, cette collaboration a davantage de chances de naître et de croître entre l'école et les parents d'un enfant qui ne présente aucune difficulté particulière et qui va bien au plan socioscolaire. Les parents d'un tel enfant éprouvent généralement un plus grand sentiment de compétence éducative et s'intéressent depuis longtemps à son développement tant social, affectif que scolaire. Dans ces cas, la collaboration avec l'école est d'autant plus facilitée si ces parents n'affrontent pas de barrières de communication du fait de ne pas maîtriser suffisamment la langue d'usage ou de vivre en contexte minoritaire pluriethnique (Desimone, 1999).

À ce titre, les parents des milieux désavantagés reçoivent un double message. D'une part, ils sont porteurs du problème en raison de leur manque d'implication parentale dans la vie scolaire de leur enfant; $d$ 'autre part, ils sont invités à protéger ses intérêts et à participer activement au processus de prise de décisions le concernant (Nakagawa, 2000). Les éléments sous-jacents aux difficultés inhérentes à la collaboration ne sont alors pas ciblés. L'encadrement potentiel des pratiques éducatives parentales, entre autres auprès des enfants ayant des comportements violents, 
n'est pas désigné (Vincent et Tomlinson, 1997). Or «les difficultés de comportement constituent d'importantes sources de tensions familiales et de conflits», notamment avec l'école (Claes et Comeau, 1996, p. 75). Quel que soit le milieu socioéconomique, la situation-problème d'un enfant concerné par cette problématique paraît insupportable aux yeux de ses parents (Deslandes et Royer, 1994). Les rencontres avec l'école visant à mettre en place une collaboration avec les parents peuvent facilement dériver et se centrer sur ces derniers et sur leurs pratiques éducatives. Les parents se sentent parfois blâmés et la collaboration avec l'école devient alors plus difficile à instaurer (Dubet et Martuccelli, 1996).

Par ailleurs, certains auteurs considèrent que la collaboration entre l'école et les parents s'avère problématique en raison de difficultés organisationnelles. Gareau et Sawatzky (1995) avancent comme principale barrière à la collaboration un manque de planification et de savoir-faire de la part des autorités scolaires. Lazar et Slostad (1999) ciblent plutôt la formation déficiente des enseignants et la rigidité de la culture scolaire.

La formation des enseignants semble faire peu de place à la compréhension des enjeux liés au vécu quotidien des familles. Les enseignants sont ainsi peu sensibilisés face aux différentes problématiques familiales. Quant à la rigidité de la culture scolaire, où domine l'idée que la salle de classe appartient aux enseignants, elle ne favoriserait pas l'implantation de programmes basés sur la collaboration entre l'école et les parents. Cette situation est observable malgré que nombre de guides pédagogiques en faveur de l'amélioration de la collaboration entre les milieux scolaire et familial aient été élaborés et mis à la disposition des enseignants ces dernières années (Goupil, 1997b ; Royer, Saint-Laurent, Bitaudeau et Moisan, 1995).

Pour leur part, Christenson et Sheridan (2001) relèvent quatre conditions pour atteindre une collaboration optimale entre l'école et les parents: la définition de buts communs, des attitudes positives invitant à un processus de résolution de problèmes sans blâme de la part des enseignants comme des parents, un climat de confiance et de respect mutuels, ainsi que le partage des responsabilités, incluant la prise de décisions communes concernant l'enfant.

Quant à Epstein (2001), à partir des résultats de ses nombreuses recherches, elle propose qu'une formation détaillée sur le sujet fasse partie des programmes de formation à l'enseignement, car lorsque les enseignants voient dans la collaboration avec les parents une valeur ajoutée à leur travail, ils l'intègrent plus facilement. 
D'autres auteurs accusent plutôt l'asymétrie de pouvoirs entre l'école et les parents d'être la source des problèmes de communication, rendant le dialogue difficile entre les deux milieux (Dubet et Martuccelli, 1996; Gareau et Sawatzky, 1995; Lazar et Slostad, 1999; Marcon, 1999; Montandon et Perrenoud, 1994; Vincent et Tomlinson, 1997). Montandon et Perrenoud (1994, p. 14) signalent que «l'école ne mesure pas toujours sa force». Selon Lawler et Yoon (1995), l'asymétrie de pouvoirs entre deux parties rend problématique la négociation entre celles-ci en vue d'établir des ententes. En effet, l'instance qui détient le plus de pouvoir tend à argumenter pour que l'autre partie considère le pouvoir comme étant partagé et, de là, que les ententes soient négociées en faveur de sa propre position. Quant à la partie détenant le moins de pouvoir, elle argumentera en fonction de la notion d'équité. Cette asymétrie de pouvoirs entrave le processus de collaboration et de prise de décisions entre l'école et les parents. Pour appuyer ce constat, dans une étude portant sur les conférences de cas, Bouchard, Clarkson et Tessier (1996) ont observé que $40 \%$ des intervenants ont tendance à imposer leurs opinions aux parents et que $70 \%$ des parents sont plutôt passifs dans la prise de décisions. Dans le prolongement de cette étude, Bouchard et Kalubi (2003) confirment ce constat de l'écart entre les deux milieux.

En définitive, ce court bilan sur la collaboration entre l'école et les parents nous a permis de dégager un certain nombre de facteurs de soutien et d'obstacles sous-jacents à la collaboration entre l'école et les parents. Toutefois, sans cette collaboration, il apparaît illusoire de pouvoir contrer les comportements violents chez les enfants de 3 à 9 ans, envers leurs pairs au préscolaire et au primaire.

Dans le but de mieux comprendre que la collaboration entre l'école et les parents procure à ces derniers des occasions de poser des gestes d'empowerment, il importe de circonscrire ce concept. Il importe également de cerner le contexte à l'intérieur duquel l'empowerment s'exerce.

\section{L'EMPOWERMENT ET LES GESTES POSÉS PAR LES PARENTS}

Cette quatrième partie vise à mieux connaître et comprendre le concept d'empowerment parental. Pour ce faire, des définitions permettent d'en saisir la complexité. L'empowerment se voit entre autres dans les gestes posés par les parents, et ce, en fonction de leur perception d'exercer une influence, voire un contrôle sur la situation-problème de leur enfant à l'école. 


\subsection{L'EMPOWERMENT: DÉFINITIONS}

Plusieurs perspectives se dégagent du concept d'empowerment. Elles vont d'une vision technocratique à un courant écologique, en passant par une position radicale de conscientisation (Drolet et Charpentier, 1999; Parsloe, 1995; Vincent, 1996). Un consensus se dessine néanmoins parmi les tenants d'orientations sociopolitiques différentes, pour définir minimalement le concept d'empowerment comme la prise en charge par la personne de sa situation personnelle. Or, ce concept ne se limite pas exclusivement à une prise en charge puisque les notions d'influence, de contrôle et de pouvoir en constituent l'essence même (Drolet et Charpentier, 1999; Breton, 1998; Pinderhughes, 1995; Rappaport, 1987).

La vision technocratique préconise la prise en charge des personnes, de leur autonomie à l'égard des mécanismes de soutien social, de leur responsabilisation par rapport à leurs problèmes, uniquement afin de réduire l'usage des services (MSSC, 1997). Cette vision s'avère réductrice et elle ne constitue pas de l'empowerment. Malgré leurs divergences idéologiques, les théoriciens s'accordent tout de même sur cette définition de l'empowerment, soit le sentiment ou la perception d'exercer une influence, voire un contrôle, sur les événements de sa vie (Breton, 1994; Rappaport, 1987).

L'empowerment désigne également une démarche où les personnes vivant dans des contextes défavorables acquièrent une meilleure maîtrise ou emprise, exercent une plus grande influence, voire un plus grand pouvoir, sur certains aspects de leur existence (Adams, 1996; Cameron et Cadell, 1997; Le Bossé, 1996; Vincent, 1996). Cette démarche permet aux personnes concernées de contrôler davantage les divers aspects de leur réalité, par des gestes concrets, malgré des conditions de vie limitatives et reliées à des contextes spécifiques (Le Bossé, 1995).

Le courant écologique met en évidence le partage inéquitable des ressources. Dans cette optique, il préconise l'empowerment pour une meilleure adaptation sociale. Les problèmes individuels sont contextualisés à l'intérieur de leurs situations limitatives. L'adaptation suppose une mobilisation des ressources pour et par ceux n'ayant que peu ou pas de droits dans la société (Dunst, Trivette et Johanson, 1994; Le Bossé, 1996; Zimmerman, 1995).

Par ailleurs, une position plus radicale s'élabore à partir d'une analyse sociohistorique des rapports structurels d'oppression. Partant d'une démarche de conscientisation, l'empowerment se réalise par des luttes collectives visant le changement social (Breton, 1994; Gutiérrez, 1994; Lee, 1994 ; Moreau et Léonard, 1993). Les modèles de pratiques basés sur 
ce type d'empowerment offrent des occasions aux exclus d'acquérir une voix et de s'en servir pour participer aux décisions qui affectent leur vie, dans le but d'agir pour obtenir une juste part des ressources de la société (Breton, 1998).

Pour sa part, l'empowerment psychologique tel que défini par Zimmerman (1995) se situe dans un courant écologique. Ce concept s'étudie au plan individuel et collectif, par l'analyse de ses composantes comportementales, interactionnelles et intrapersonnelles. Les composantes comportementales s'attardent aux gestes d'adaptation et de résistance. Quant aux composantes interactionnelles, elles s'intéressent aux habiletés personnelles à s'affirmer, à résoudre des situations-problèmes, à prendre des décisions et à mobiliser des ressources afin d'améliorer ses conditions de vie, de même qu'à exercer une compréhension critique du contexte social. Pour ce qui est des composantes intrapersonnelles, elles mettent l'accent sur les résultats d'une démarche d'empowerment, soit le contrôle interne, l'efficacité personnelle, le pouvoir d'influence et la compétence. Le contrôle interne suppose chez la personne la perception d'un lien entre les résultats d'une action et ses capacités, ses caractéristiques et ses comportements (Kieffer, 1984; Zimmerman et Rappaport, 1988; Zimmerman, 1995). En ce qui a trait à l'efficacité personnelle, elle renvoie à la confiance qu'ont les personnes dans leur capacité de prendre les mesures nécessaires pour atteindre leurs objectifs (Ozer et Bandura, 1990 ; Kieffer, 1984 ; Zimmerman et Rappaport, 1988; Zimmerman, 1995). Quant au pouvoir d'influence d'une personne, il se traduit par la capacité d'agir sur les forces qui affectent sa vie ou de produire des effets désirés chez les autres (Pinderhughes, 1995), alors que la compétence désigne une reconnaissance par autrui de ses accomplissements personnels (Dangel et Polster, 1984), voire de son savoir-agir (Lasnier, 2000, p. 31). À ce titre, le sentiment de compétence tel que défini par Hoover-Dempsey, Bassler et Brissie (1992), comme facteur de soutien à l'implication des parents dans la vie scolaire de leur enfant, fait directement appel à cette dimension de l'empowerment.

En somme, l'empowerment psychologique comprend une interaction dynamique entre les composantes comportementales, interactionnelles et intrapersonnelles. Puisqu'il s'agit d'un processus fondé sur l'exercice d'une influence, voire d'un contrôle sur sa propre vie, une telle interaction s'inscrit dans un partenariat égalitaire (Dunst, Trivette et Johanson, 1994; Gutiérrez, DeLois et GlenMaye, 1995; Kay, Fitzgerald et McConaughy, 1997; Kieffer, 1984; Moreau et Léonard, 1993). 


\subsection{LES GESTES D'EMPOWERMENT POSÉS PAR LES PARENTS}

Montandon et Perrenoud (1994) insistent sur l'importance des représentations dans les relations entre l'école et les parents. Le concept de représentation désigne ici «l'organisation personnelle du savoir d'un individu qui tend à l'élaboration d'un système explicatif et opératoire, mettant en jeu des catégories et des relations particulières, pour structurer son expérience personnelle» (Ackerman et Rialan, cités dans Schiele et Boucher, 1997, p. 429). Quant au concept de perception, il renvoie aux représentations conscientes d'un individu à partir de ses sensations (Le Petit Larousse, 1999). Puisque les parents perçoivent l'école, comme institution, à travers les contacts et les échanges qu'ils entretiennent avec les individus y œuvrant (personnel enseignant, direction d'école, personnel non enseignant, etc.), il convient davantage d'utiliser le concept de perception dans le présent ouvrage pour décrire les représentations qu'ils se font de l'école.

À partir de ces représentations, le savoir se construit. Tel que Montandon et Perrenoud (1994, p. 21) l'avancent: «Les interactions entre l'école et la famille dépendent grandement de leurs représentations réciproques. » Et, dans la foulée de ce constat, Jodelet (1997, p. 59) croit qu'elles permettent l'émergence d'une «forme de savoir pratique». Chaque acteur adopte ainsi des positions lors de la prise de contact, lorsque des négociations s'entament entre les deux parties. De ces échanges peut émerger ou non une collaboration. Toutefois, «à partir d'une conception stratégique de l'action, chacun est orienté par ses propres intérêts, la représentation $q^{\prime}$ 'il en a et par les règles de l'organisation [...]. Le tout est fonction d'un pouvoir lié à sa capacité de négociation» (Dubet, 1994, p. 86). Bref, les ressources auxquelles chaque acteur a accès, l'autorité découlant de l'organisation structurelle et la force de persuasion jouent un rôle crucial dans la capacité d'accepter ou de refuser les demandes émanant de l'autre (Crozier et Friedberg, 1977).

Or, dans le contexte de l'asymétrie de pouvoir entre l'école et les parents, le fait pour le personnel scolaire d'agir au plan individuel tout en représentant les intérêts collectifs de l'institution complique le dialogue. Cette situation tend à personnaliser les entraves institutionnelles. C'est pourquoi il importe aux parents de faire le partage entre ce qui revient aux personnes et aux institutions, afin d'éviter une individualisation des contextes. Ce partage est d'autant plus important lorsqu'apparaissent les difficultés qu' ont les parents avec l'école, en raison de tensions élevées liées à leurs divergences de positions au sujet de la situation-problème de leur enfant. Ces éléments contextualisent les enjeux de la collaboration entre l'école et les parents. Dans un tel contexte d'asymétrie de pouvoir, les parents peuvent se voir offrir des occasions de poser des gestes d'empowerment, à 
savoir des actions concrètes leur permettant d'exercer une influence, voire un contrôle sur leur réalité familiale et sur celle de leur enfant à l'école. Les gestes d'adaptation que peut poser un individu visent à maîtriser, tolérer et diminuer l'impact d'un événement stressant sur le système familial (Desmarais, Beauregard, Guérette, Hrimech, Lebel, Martineau et Péloquin, 2000 ; Paulhan et Bourgeois, 1995). Les parents peuvent également s'opposer aux décisions déjà prises par l'école. L'opposition implique l'action de faire obstacle à quelqu'un ou quelque chose. Par des gestes d'opposition, les parents affirment leur autorité en contrecarrant les décisions prises à leur encontre. Les gestes d'adaptation et d'opposition font appel à la gestion des émotions et, encore plus, à l'analyse et à la résolution de problèmes. Ils rejoignent la démarche d'empowerment psychologique, telle que définie par Zimmerman (1995), par la mise en action personnelle et par la mobilisation de ressources permettant d'exercer une influence positive, voire de surmonter, la situation-problème de l'enfant.

Par ailleurs, lors de décisions imposées, les parents peuvent poser des gestes de résistance (Ashforth et Mael, 1998). La résistance est définie comme étant «des actes ou des omissions intentionnels qui défient les désirs des autres» (Ashforth et Mael, 1998, p. 90). Selon Rooney (1992), il peut s'agir de gestes posés par des clients dans un contexte non volontaire, soit des comportements qui ne vont pas dans le sens des attentes. La résistance peut également consister en des pratiques silencieuses de protestation, soit des gestes isolés, fragmentés, peu visibles dans le contexte d'une relation de pouvoir où le client ne peut pas véritablement se désengager (Trethewey, 1997). Elle peut également se traduire par des tactiques visant à aller chercher des gains (Cohen, 1998). L'humour, le retrait, la conformité, la manipulation, la dépendance deviennent alors des occasions de poser des gestes d'empowerment (Pinderhughes, 1995). Pour sa part, Van Zenten (2001) reconnaît également qu'il peut s'agir d'accords tacites ou de défections, des gestes posés par les parents afin d'exercer une influence, voire un contrôle sur leur réalité. Dans le même esprit, Vatz-Laaroussi (1996) parle de gestes de survie posés dans le but de se protéger (Dubet et Martuccelli, 1996).

En définitive, dans le cadre du présent ouvrage, la forme de pouvoir que les parents peuvent exercer par rapport à l'école correspond à leur capacité d'influencer les forces qui affectent leur vie ainsi que celle de leur enfant à l'école afin d'obtenir les effets désirés (Pinderhughes, 1995). Dans le contexte relatif à la collaboration entre l'école et les parents, l'empowerment est défini comme étant la perception qu'ont les parents d'exercer une influence, voire un contrôle sur les situations-problèmes et les événements (Rappaport, 1987). Les parents sont considérés comme des partenaires qui prennent une place et la parole et qui participent d'une façon ou d'une 
autre. Ils peuvent négocier avec le milieu scolaire, à partir de leurs perceptions, de leurs intérêts et de ceux de leur enfant. Ils peuvent également poser des gestes d'adaptation, d'opposition ou de résistance par rapport aux propositions de l'école. Tout ce contexte est propice à leur fournir des occasions de poser des gestes d'empowerment. En outre, l'analyse et la résolution de la situation-problème ainsi que la mobilisation de ressources deviennent des indicateurs de la mise en œuvre de l'empowerment, qui s'exerce par les gestes que posent les parents. Cette perspective soulève une autre des questions secondaires de la présente recherche à savoir quels gestes d'empowerment posés par les parents leur permettent d'exercer une influence, voire un contrôle sur la situation-problème de leur enfant à l'école? Nous explorerons cette question sous l'angle des perceptions des parents concernés par la problématique des comportements violents de leur enfant de 3 à 9 ans envers leurs pairs au préscolaire et au primaire.

Avant toutefois d'aborder les résultats relatifs aux perceptions parentales, nous consacrerons le prochain chapitre à la présentation du portrait des enfants et des parents qui sont les sujets de la présente recherche. 


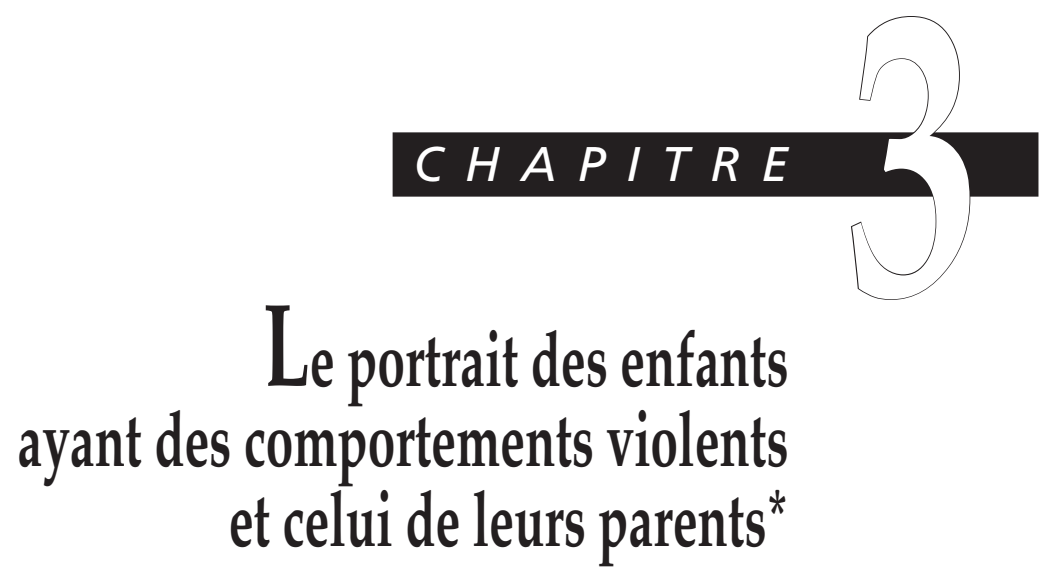

Des extraits de ce chapitre sont tirés de: R. Hasan, M. Drolet et M. Paquin (2003). «Les conduites violentes chez les 3 à 6 ans: comprendre pour mieux intervenir", Reflet, Revue ontaroise d'intervention sociale et communautaire, 9(1), p. 150-177. 

Le troisième chapitre de cet ouvrage présente le portrait des enfants ayant des comportements violents selon les perceptions des parents ayant participé à la recherche. Dans une première partie, nous décrivons la méthodologie ayant servi à recueillir les données auprès de soixante parents d'enfants de 3 à 9 ans ayant des comportements violents envers leurs pairs au préscolaire et au primaire. Cette partie enchaîne avec les résultats portant sur les perceptions parentales au sujet des comportements violents de leur enfant, des divers problèmes associés, de même que sur la durée, la fréquence, le lieu, le moment et la trajectoire de ces comportements. Puis, nous présentons les résultats relatifs aux pratiques éducatives parentales ayant un effet positif et négatif, aux stresseurs familiaux affectant négativement les comportements de l'enfant, ainsi qu'aux facteurs explicatifs de sa situation-problème à l'école. Les résultats sont d'abord présentés pour le groupe des parents d'enfants de 3 à 6 ans, qui sont au préscolaire, puis pour celui des parents d'enfants de 6 à 9 ans, qui sont au primaire. Le chapitre se termine par un bilan brossant le portrait de l'ensemble des sujets de l'échantillon.

\section{LA MÉTHODOLOGIE DE RECHERCHE}

Cette première partie présente les questions de recherche, principale et secondaires, auxquelles nous nous sommes intéressées. Puis, nous exposons les objectifs de la recherche découlant de ces questions. Par la suite, nous décrivons les différentes étapes de la recherche, soit la collecte, le traitement, l'analyse et l'interprétation des données. Finalement, nous dressons le portrait des enfants et de leurs parents ayant participé à la collecte de données et nous établissons les limites de la recherche.

\subsection{LES QUESTIONS ET LES OBJECTIFS DE LA RECHERCHE}

Les questions de recherche ont été formulées dans la foulée des constats les plus récents portant sur la problématique des enfants ayant des comportements violents envers leurs pairs au préscolaire et au primaire. De cette problématique découle une meilleure connaissance et compréhension du portrait des enfants et de leurs parents, du contexte de collaboration et de non-collaboration avec l'école, des facteurs de soutien et des obstacles sous-jacents, des gestes d'empowerment posés par les parents afin d'exercer une influence, voire un contrôle sur la situation-problème de leur enfant à l'école, ainsi que des diverses mesures disciplinaires appliquées par l'école en réponse aux comportements violents des enfants envers leurs pairs au préscolaire et au primaire. Les questions de recherche principale et secondaires sont présentées au tableau 3.1. 


\section{TABleau 3.1}

\section{Les questions de recherche, principale et secondaires}

\begin{tabular}{ll}
$\begin{array}{l}\text { Question } \\
\text { principale }\end{array}$ & $\begin{array}{l}\text { Quels sont les défis et les enjeux de la collaboration entre l'école } \\
\text { et les parents d'enfants de } 3 \text { et } 9 \text { ans afin de contrer leurs compor- } \\
\text { tements violents envers leurs pairs au préscolaire et au primaire? }\end{array}$ \\
\hline $\begin{array}{l}\text { Questions } \\
\text { secondaires }\end{array}$ & $\begin{array}{l}\text { 1) Quel est le portrait des parents et des enfants de } 3 \text { à } 9 \text { ans } \\
\text { ayant des comportements violents envers leurs pairs au } \\
\text { préscolaire et au primaire? }\end{array}$ \\
& 2) Quels sont les éléments constituant un contexte de collabora- \\
tion et de non-collaboration entre l'école et les parents de ces \\
enfants? \\
3) Quels sont les facteurs de soutien et les obstacles sous-jacents à \\
la collaboration entre l'école et les parents de ces enfants? \\
4) Quels sont les gestes d'empowerment posés par les parents leur \\
permettant d'exercer une influence, voire un contrôle sur la \\
situation-problème de leur enfant à l'école? \\
5) Quelles sont les mesures disciplinaires appliquées par l'école \\
permettant de contrer les comportements violents des 3 à 9 ans \\
envers leurs pairs au préscolaire et au primaire et de ne pas \\
entraver leur processus de scolarisation et de socialisation?
\end{tabular}

Ces questions ont donné lieu à l'identification de sept objectifs de recherche ayant constitué chacune des parties de la grille d'entrevue parentale. Les objectifs sont présentés au tableau 3.2.

\section{TABLEAU 3.2}

\section{Les sept objectifs de recherche}

1. Cerner les perceptions des parents au sujet des comportements violents de leur enfant envers leurs pairs au préscolaire et au primaire.

2. Décrire la problématique des parents et de leur enfant de 3 à 9 ans ayant des comportements violents envers leurs pairs au préscolaire et au primaire.

3. Explorer les pratiques éducatives parentales ayant un effet positif et négatif sur les comportements violents de leur enfant.

4. Tracer la trajectoire de la collaboration entre l'école et les parents et exposer les facteurs de soutien et les obstacles sous-jacents.

5. Cerner les perceptions des parents au sujet de l'influence, voire le contrôle qu'ils exercent sur la situation-problème de leur enfant à l'école.

6. Dégager les occasions permettant aux parents de poser des gestes d'empowerment pour améliorer leur situation familiale et celle de leur enfant à l'école.

7. Cerner les perceptions des parents face à la suspension scolaire ainsi qu'aux autres mesures disciplinaires appliquées par l'école en réponse aux comportements violents de leur enfant envers leurs pairs au préscolaire et au primaire. 
L'atteinte de l'ensemble des objectifs permet de répondre à la question principale de recherche. Pour ce qui est de l'atteinte des trois premiers objectifs, elle permet de répondre à la première question secondaire. Quant à l'atteinte de l'objectif 4, elle permet de répondre aux deuxième et troisième questions secondaires. Puis, l'atteinte des objectifs 5 et 6 permet de répondre à la quatrième question secondaire. Finalement, l'atteinte de l'objectif 7 permet de répondre à la cinquième question secondaire de recherche.

\subsection{LES ÉTAPES DE LA RECHERCHE}

Afin d'apporter des pistes de réponses aux questions de recherche, nous avons effectué des entrevues auprès de soixante parents d'enfants de 3 à 9 ans ayant des comportements violents envers leurs pairs au préscolaire et au primaire. Pour faire partie de l'échantillon, l'enfant devait faire l'objet d'une suspension ou d'une possibilité de suspension scolaire en réponse à de tels comportements. Dans ce contexte, il incombait au milieu scolaire de juger de la teneur des comportements et de déterminer s'ils étaient inappropriés au sens de la Loi sur la sécurité dans les écoles (Ministère de l'Éducation de l'Ontario, 2000) et du Code de conduite provincial (Ministère de l'Éducation de l'Ontario, 2001a).

Le recrutement des participants à la recherche s'est fait auprès des deux conseils scolaires de langue française de la région d'Ottawa, ainsi qu'auprès d'organismes communautaires francophones, soit le Centre psychosocial pour enfants et familles et le Centre de ressources de la Basse-ville. Finalement, quelques parents ont participé à la recherche à la suite d'une invitation publique parue dans les journaux locaux et régionaux. L'échantillon est constitué de parents qui se sont tous portés volontaires (Mayer, Ouellet, Saint-Jacques et Turcotte, 2000) et qui ont signé un formulaire de consentement libre et éclairé les informant de leurs droits et responsabilités. Une fois leur consentement obtenu, les parents ont participé à une entrevue téléphonique. Les membres de l'équipe de recherche ont élaboré une grille pour la réalisation de l'entrevue. La grille d'entrevue parentale comporte sept parties se rapportant à chacun des objectifs de recherche identifiés au tableau 3.2. Une fois la grille d'entrevue parentale élaborée, elle a été soumise individuellement à des enseignants, des directeurs d'école, des directeurs de services à l'élève, des psychologues et des travailleurs sociaux, recrutés dans le milieu scolaire et communautaire. La mise en commun des commentaires formulés par ceux-ci a permis d'apporter des modifications par consensus à la version initiale de la grille d'entrevue. Cette version modifiée a ensuite fait l'objet d'une préexpérimentation auprès de six parents qui ont été exclus de l'échantillon 
de recherche. À la suite de quoi, des corrections mineures ont été apportées dans une version finale qui a été soumise aux soixante parents constituant l'échantillon final. La version finale de la grille d'entrevue parentale est présentée à l'annexe 1 .

En ce qui a trait à la collecte des données, la moitié des parents ont réalisé une entrevue structurée et l'autre, une entrevue semi-structurée, le tout en fonction des disponibilités de chaque participant. La durée des entrevues varie de trente minutes à deux heures. Les entrevues téléphoniques ont été enregistrées, puis transcrites sur traitement de texte.

En ce qui concerne le traitement des données, le contenu des entrevues transcrites a été dépouillé et codifié à l'aide du logiciel d'analyse qualitative $\mathrm{N}-\mathrm{VIVO}$ (version 1.3). Le dépouillement et la codification ont été effectués en fonction des unités de sens qui se dégageaient des propos des parents (Mayer, Ouellet, Saint-Jacques et Turcotte, 2000). Afin d'y parvenir, nous avons effectué une première lecture en collaboration avec notre assistante de recherche. Cette première lecture dite «horizontale» des unités de sens consistait à repérer les thèmes, les divergences et les convergences qui émanaient du contenu de certaines entrevues ciblées pour leur profondeur. Cette étape a permis d'assurer une cohérence interne au dépouillement des unités de sens, en plus de nous permettre d'élaborer une grille contenant des catégories d'analyse. Une fois la grille d'analyse des unités de sens complétée, une deuxième lecture dite «verticale» a permis de bonifier les catégories (Huberman et Miles, 1991). Afin d'assurer la validité de la codification, quelques entrevues codifiées ont fait l'objet d'un calcul de l'indice $\mathrm{d}^{\prime}$ accord interjuges. Cette étape a ensuite permis à l'assistante de recherche de procéder de manière autonome au dépouillement et à la codification de l'ensemble des entrevues de recherche. La grille des catégories d'analyse est présentée à l'annexe 2 .

L'ensemble des entrevues codifiées ont ensuite été classifiées selon les deux groupes d'âge des enfants, soit les 3 à 6 ans, correspondant au préscolaire, et les 6 à 9 ans, correspondant au primaire. Puis, ces deux regroupements ont été classifiés en fonction du milieu socioéconomique des familles, soit les parents vivant en contexte de pauvreté et ceux des classes moyenne et moyenne-supérieure. De plus, selon le milieu socioéconomique, nous avons accordé une attention particulière au niveau de scolarité des parents. En effet, les deux variables sociodémographiques que sont le milieu socioéconomique des familles et le niveau de scolarité des parents s'avèrent de précieux facteurs prédicteurs de la collaboration entre l'école et les parents (Dubet et Martuccelli, 1996; Epstein, 1995; Grolnick, Gurland, Jacob et Decourcey, 1997; Lareau, 1987; Marcon, 1999). 
L'analyse des données a été effectuée de manière inductive (L'Écuyer, 1985). Afin d'y parvenir, les chercheures et l'assistante de recherche ont entrepris une troisième lecture, soit un va-et-vient entre les données codifiées et le contenu des entrevues. Cette étape a permis de procéder à l'interprétation des données qui portaient davantage sur la mise en perspective des propos à travers le contenu global des entrevues.

Finalement, l'utilisation du logiciel N-VIVO (version 1.3) nous a permis de procéder à une quantification des participants. Des tableaux récapitulatifs sont présentés essentiellement dans le but de faciliter la lecture des résultats de l'analyse qualitative. Cette quantification comprend des nombres et des pourcentages. À des fins pratiques, ces derniers sont arrondis à 0,5 .

\subsection{LES DONNÉES SOCIODÉMOGRAPHIQUES}

L'échantillon se compose de soixante parents ayant participé à la recherche. Les données sociodémographiques présentent l'âge, le statut familial et le milieu socioéconomique des familles, l'occupation, le niveau de scolarité et la langue parlée à la maison.

\subsubsection{L'âge des parents}

Neuf parents sur dix sont des mères, dont la moyenne d'âge se situe entre 30 et 34 ans. L'échantillon compte également une grand-mère adoptive qui a entre 50 et 54 ans. Quant aux pères, au nombre de six, leur moyenne d'âge se situe entre 40 et 44 ans. Une seule mère a entre 20 et 24 ans et aucun père n'a moins de 20 ans. Par conséquent, cet échantillon rejoint très peu les jeunes parents. Par ailleurs, cinq mères ont entre 45 et 49 ans, ce qui signifie qu'elles ont accouché de l'enfant concerné autour de la quarantaine. Autre fait à signaler, les pères répondants de l'échantillon ont tous un fils âgé de 6 à 9 ans. La répartition des parents selon le groupe d'âge est présentée au tableau 3.3. 
TABLEAU 3.3

La répartition des parents selon le groupe d'âge $(n=60)$

\begin{tabular}{lrrrr}
\hline & \multicolumn{2}{c}{ Mères } & \multicolumn{2}{c}{ Pères } \\
\cline { 2 - 5 } & $\boldsymbol{N}$ & $\%$ & $\boldsymbol{N}$ & $\%$ \\
\cline { 2 - 5 } & 54 & 90,0 & 6 & 10,0 \\
\hline $20-24$ ans & 1 & 1,5 & & \\
\hline $25-29$ ans & 13 & 22,0 & & \\
\hline $30-34$ ans & 16 & 27,0 & 1 & 1,5 \\
\hline $35-39$ ans & 9 & 15,0 & 1 & 1,5 \\
\hline $40-44$ ans & 9 & 15,0 & 2 & 3,5 \\
\hline $45-49$ ans & 5 & 8,0 & 1 & 1,5 \\
\hline $50-54$ ans & 1 & 1,5 & 1 & 1,5 \\
\hline
\end{tabular}

\subsubsection{Le statut familial et le milieu socioéconomique des familles}

Au plan du statut familial, deux familles sur cinq sont intactes, près de la moitié sont monoparentales et dix pour cent sont reconstituées ou recomposées, à savoir que ces dernières ont un enfant commun aux nouveaux conjoints. Parmi les familles monoparentales, 27 sont dirigées par une mère seule et deux par un père seul.

En ce qui concerne le milieu socioéconomique, selon le Conseil national du bien-être social (2002), 23 familles vivent dans un contexte de pauvreté ${ }^{1}, 12$ se situent dans la classe moyenne et 25 se situent dans les classes moyenne et moyenne-supérieure. En concordance avec les résultats des plus récentes études sur la pauvreté au Canada (Conseil national du bien-être social, 2002), 18 des 23 familles qui vivent en contexte de pauvreté sont également monoparentales. Sur ces 18 familles, une seule est dirigée par un père seul. Par ailleurs, six des familles à haut revenu sont dirigées par une mère seule. Ce qui signifie que monoparentalité ne rime pas toujours avec pauvreté. Le statut des familles en fonction des tranches de revenu annuel est présenté au tableau 3.4.

1. Le statut familial est déterminé par le nombre de membres en fonction du revenu annuel. 
Tableau 3.4

Le statut des familles en fonction des tranches de revenu annuel $(n=60)$

\begin{tabular}{|c|c|c|c|c|c|c|c|c|}
\hline & \multirow{2}{*}{\multicolumn{2}{|c|}{ Intactes }} & \multicolumn{4}{|c|}{ Monoparentales } & \multirow{2}{*}{\multicolumn{2}{|c|}{$\begin{array}{c}\text { Reconstituées/ } \\
\text { Recomposées }\end{array}$}} \\
\hline & & & \multicolumn{2}{|c|}{ Mères } & \multicolumn{2}{|c|}{ Pères } & & \\
\hline & $N$ & $\%$ & $N$ & $\%$ & $N$ & $\%$ & $N$ & $\%$ \\
\hline & 25 & 42,0 & 27 & 45,0 & 2 & 3,5 & 6 & 10,0 \\
\hline Moins de $20000 \$$ & & & 9 & 15,0 & 1 & 1,5 & 1 & 1,5 \\
\hline $20000 \$$ à $24999 \$$ & & & 4 & 6,5 & & & 1 & 1,5 \\
\hline $25000 \$$ à $29999 \$$ & 1 & 1,5 & 1 & 1,5 & & & 1 & 1,5 \\
\hline $30000 \$$ à $34999 \$$ & 3 & 5,0 & 4 & 6,5 & & & & \\
\hline $35000 \$$ à $39999 \$$ & 1 & 1,5 & 1 & 1,5 & 1 & 1,5 & & \\
\hline $40000 \$$ à $44999 \$$ & 1 & 1,5 & 1 & 1,5 & & & & \\
\hline $45000 \$$ à $49999 \$$ & 3 & 5,0 & 1 & 1,5 & & & & \\
\hline $50000 \$$ à $59999 \$$ & 4 & 6,5 & 3 & 5,0 & & & & \\
\hline $60000 \$$ à $74999 \$$ & 1 & 1,5 & 3 & 5,0 & & & 1 & 1,5 \\
\hline $75000 \$$ et plus & 11 & 19,0 & & & & & 2 & 3,5 \\
\hline
\end{tabular}

\subsubsection{L'occupation des parents}

Trente-neuf mères sur 54 sont à temps plein ou partiel sur le marché du travail, trois sont aux études à temps plein ou partiel, trois sont à la recherche d'un emploi et huit demeurent à la maison. Quant à la grandmère, elle demeure également à la maison. Parmi les pères, quatre occupent un emploi à temps plein, un est à la recherche d'un emploi et l'occupation du dernier père est indéterminée. Par conséquent, quatre parents sur cinq de cet échantillon sont réputés faire partie de la population active. L'occupation des parents est présentée au tableau 3.5. 
TABleau 3.5

L'occupation des parents $(n=60)$

\begin{tabular}{|c|c|c|c|c|}
\hline & & & & \\
\hline & $N$ & $\%$ & $N$ & $\%$ \\
\hline & 54 & 90,0 & 6 & 10,0 \\
\hline Travail à temps plein & 33 & 55,0 & 4 & 6,5 \\
\hline À la maison & 9 & 15,5 & & \\
\hline Travail à temps partiel & 6 & 10,0 & & \\
\hline Recherche d'emploi & 3 & 5,0 & 1 & 1,5 \\
\hline Études à temps plein & 2 & 3,5 & & \\
\hline Études à temps partiel & 1 & 1,5 & & \\
\hline Indéterminée & & & 1 & 1,5 \\
\hline
\end{tabular}

\subsubsection{Le niveau de scolarité des parents}

Quinze mères possèdent un diplôme d'études universitaires, 14 un diplôme d'études collégiales, 23 un diplôme d'études secondaires et deux n'ont terminé que leurs études élémentaires. Quant aux pères, deux possèdent un diplôme d'études universitaires, deux un diplôme d'études collégiales, un a un diplôme d'études secondaires et le niveau de scolarité du dernier père est indéterminé. Dans cet échantillon, malgré le fait que le quart des mères détiennent un diplôme universitaire, en moyenne, leur niveau de scolarité est moins élevé que celui des pères. Le niveau de scolarité des parents est présenté au tableau 3.6.

TABleau 3.6

Le niveau de scolarité des parents $(n=60)$

\begin{tabular}{lrccc}
\hline & \multicolumn{2}{c}{ Mères } & \multicolumn{2}{c}{ Pères } \\
\cline { 2 - 5 } & $\boldsymbol{N}$ & $\%$ & $\boldsymbol{N}$ & $\%$ \\
\cline { 2 - 5 } & 54 & 90,0 & 6 & 10,0 \\
\hline Élémentaire & 2 & 3,5 & & \\
\hline Secondaire & 23 & 38,5 & 1 & 1,5 \\
\hline Collégial & 14 & 23,5 & 2 & 3,5 \\
\hline Université & 15 & 25,0 & 2 & 3,5 \\
\hline Indéterminé & & & 1 & 1,5 \\
\hline
\end{tabular}




\subsubsection{La langue parlée à la maison}

La moitié des familles déclarent parler français à la maison. Le quart des familles déclarent parler le français et l'anglais. Finalement, sept familles déclarent parler le français ainsi qu'une autre langue, soit le créole, l'espagnol, le jamaïcain ou une langue africaine. Toutefois, sur ce dernier nombre, quatre ne maîtrisent ni le français, ni l'anglais, parce qu'elles sont nouvellement arrivées au pays. De plus, ces sept familles vivent toutes en contexte de pauvreté. La langue parlée à la maison est présentée au tableau 3.7.

TABleau 3.7

La langue parlée à la maison $(n=60)$

\begin{tabular}{lcccc}
\hline & \multicolumn{2}{c}{ Mères $(\boldsymbol{n}=\mathbf{5 4})$} & \multicolumn{2}{c}{ Pères $(\boldsymbol{n}=\mathbf{6})$} \\
\cline { 2 - 5 } & $\boldsymbol{N}$ & $\boldsymbol{\%}$ & $\boldsymbol{N}$ & $\boldsymbol{\%}$ \\
\cline { 2 - 5 } & 54 & 90,0 & 6 & 10,0 \\
\hline Français & 25 & 42,5 & 4 & 6,5 \\
\hline Français et anglais & 23 & 38,0 & 1 & 1,5 \\
\hline Français et créole & 3 & 5,0 & & \\
\hline Français et espagnol & 2 & 3,5 & & \\
\hline Français et jamaïcain & 1 & 1,5 & & \\
\hline Français et africain & & & 1 & 1,5 \\
\hline
\end{tabular}

\subsection{LES DONNÉES SOCIODÉMOGRAPHIQUES DES ENFANTS DE 3 À 9 ANS}

L'échantillon d'enfants se compose de 54 garçons et de six filles. Cette répartition entre les sexes, soit neuf garçons pour une fille, correspond à la situation généralement décrite dans les écrits sur la question, à savoir que la problématique des comportements violents est davantage présente chez le sexe masculin que féminin (Kauffman, 1997). Les données sociodémographiques présentent l'âge, le statut dans la famille, le niveau scolaire, les antécédents scolaires et médicaux.

\subsubsection{L'âge des enfants}

Dans le regroupement des 3 à 6 ans, il y a 23 enfants, soit 20 garçons et trois filles, tandis que dans celui des 6 à 9 ans, il y en a 37, soit 34 garçons et trois filles. Le plus grand nombre d'enfants de 6 à 9 ans, comparativement à ceux 
des 3 à 6 ans, semble concorder avec le fait que plus ils vieillissent, plus les comportements violents s'aggravent (Loeber et Farrington, 2000). La répartition des enfants selon le groupe d'âge est présentée au tableau 3.8.

TABLEAU 3.8

La répartition des enfants selon le groupe d'âge $(n=60)$

\begin{tabular}{lrrrr}
\hline & \multicolumn{2}{c}{ Garçons } & \multicolumn{2}{c}{ Filles } \\
\cline { 2 - 5 } & $\boldsymbol{N}$ & $\%$ & $\boldsymbol{N}$ & $\%$ \\
\cline { 2 - 5 } & 54 & 90,0 & 6 & 10,0 \\
\hline 3 à 4 ans & 3 & 5,0 & 1 & 1,5 \\
\hline 4 à 5 ans & 6 & 10,0 & 1 & 1,5 \\
\hline 5 à 6 ans & 11 & 18,5 & 1 & 1,5 \\
\hline 6 à 7 ans & 9 & 15,0 & 3 & 5,0 \\
\hline 7 à 8 ans & 11 & 18,5 & & \\
\hline 8 à 9 ans & 14 & 23,0 & & \\
\hline
\end{tabular}

\subsubsection{Le statut des enfants dans la famille}

Des soixante enfants, un peu plus du tiers sont uniques, alors qu'un peu moins du tiers sont les benjamins d'une famille comprenant de deux à sept enfants. Également, le quart d'entre eux sont les aînés d'une famille comprenant deux ou trois enfants. Finalement, un sur dix est le cadet d'une famille, en se situant soit entre le premier et le troisième enfant de la famille, soit entre le deuxième et le quatrième enfant. Le statut des enfants dans la famille est présenté au tableau 3.9.

TABleAu 3.9

Le statut des enfants dans la famille $(n=60)$

\begin{tabular}{lcccr}
\hline & \multicolumn{2}{c}{ Garçons } & \multicolumn{2}{c}{ Filles } \\
\cline { 2 - 5 } & $\boldsymbol{N}$ & $\%$ & $\boldsymbol{N}$ & $\%$ \\
\cline { 2 - 5 } & 54 & 90,0 & 6 & 10,0 \\
\hline Unique & 20 & 33,5 & 1 & 1,5 \\
\hline Benjamin & 16 & 26,5 & 2 & 3,5 \\
\hline Aîné & 12 & 20,0 & 3 & 5,0 \\
\hline Cadet & 6 & 10,0 & & \\
\hline
\end{tabular}




\subsubsection{Le niveau scolaire des enfants}

Des soixante enfants, 23 sont au préscolaire, soit quatre à la garderie, sept à la maternelle et 12 au jardin. Par ailleurs, 37 enfants sont au primaire, soit 12 en première année, 15 en deuxième année et dix en troisième année. Parmi eux, un garçon et une fille ont doublé leur première année, trois garçons ont doublé leur deuxième année et quatre garçons ont doublé leur troisième année. Le niveau scolaire des 3 à 9 ans est présenté au tableau 3.10.

Tableau 3.10

Le niveau scolaire des 3 à 9 ans* $(n=60)$

\begin{tabular}{lrrrr}
\hline & \multicolumn{2}{c}{ Garçons } & \multicolumn{2}{c}{ Filles } \\
\cline { 2 - 5 } & $\boldsymbol{N}$ & $\%$ & $\boldsymbol{N}$ & $\%$ \\
\cline { 2 - 5 } & 54 & 90,0 & 6 & 10,0 \\
\hline Garderie & 3 & 5,0 & 1 & 1,5 \\
\hline Maternelle & 6 & 10,0 & 1 & 1,5 \\
\hline Jardin & 11 & 18,5 & 1 & 1,5 \\
\hline Première année & 10 & 16,5 & 2 & 3,5 \\
\hline Deuxième année & 14 & 23,0 & 1 & 1,5 \\
\hline Troisième année & 10 & 16,5 & & \\
\hline
\end{tabular}

\subsubsection{Les antécédents scolaires des enfants}

Deux enfants sur cinq éprouvent des difficultés d'apprentissage dans certaines matières, notamment en lecture, en écriture et en mathématiques. Ces difficultés nécessitent la mise en œuvre d'un Plan d'enseignement individualisé (PEI) et d'un suivi de la part d'un orthopédagogue (en classe-ressource) ou d'un éducateur spécialisé (en classe régulière). Ainsi, le doublement et les difficultés d'apprentissage riment souvent avec les difficultés de comportement (Bender et Smith, 1990 ; Fortin, Toupin, Pauzé, Déry et Mercier, 1996; Mishna, 2003). Par ailleurs, dix enfants ont bénéficié d'un placement dans une école et une classe spéciales, aux termes de la «Section 20» de la Loi sur l'éducation de l'enfance en difficulté (Ministère de l'Éducation de l'Ontario, 2001b). Les antécédents scolaires des 3 à 9 ans sont présentés au tableau 3.11.

* En Ontario, les enfants admis au préscolaire peuvent débuter la maternelle dès l'âge de 4 ans. S'ils sont inscrits à temps complet, ils fréquenteront le Jardin d'enfant l'année suivante, puis commenceront le primaire en $1^{\text {re }}$ année. S'ils sont inscrits à temps partiel, ils fréquenteront soit une garderie, soit un service de garde en milieu scolaire pour la portion de la journée où ils ne sont pas en classe. Garderie $=3 \mathrm{ou}$ 4 ans; Maternelle $=4$ ou 5 ans; Jardin $=5$ ans; $1^{\text {re }}$ année $=6$ ans, etc. 
TABLEAU 3.11

Les antécédents scolaires des 3 à 9 ans $(n=60)$

\begin{tabular}{lcccc}
\hline & \multicolumn{2}{c}{ Garçons } & \multicolumn{2}{c}{ Filles } \\
\cline { 2 - 5 } & $N$ & $\%$ & $N$ & $\%$ \\
\cline { 2 - 5 } & 54 & 90,0 & 6 & 10,0 \\
\hline $\begin{array}{l}\text { Difficultés d'apprentissage } \\
\text { dans les matières }\end{array}$ & 21 & 35,0 & 2 & 3,5 \\
\hline $\begin{array}{l}\text { Plan d'enseignement } \\
\text { individualisé (PEI) }\end{array}$ & 17 & 28,0 & 1 & 1,5 \\
\hline $\begin{array}{l}\text { Suivi d'un orthopédagogue } \\
\text { (en classe-ressource) }\end{array}$ & 16 & 26,5 & 2 & 3,5 \\
\hline $\begin{array}{l}\text { École et classe spéciales } \\
\text { (Section 20) }\end{array}$ & 8 & 13,0 & 2 & 3,5 \\
\hline $\begin{array}{l}\text { Suivi d'un éducateur spécialisé } \\
\text { (classe régulière) }\end{array}$ & 1 & 1,5 & & \\
\hline
\end{tabular}

\subsubsection{Les antécédents médicaux des enfants}

Quant à leurs antécédents médicaux, le quart des enfants ont un diagnostic du Trouble du déficit de l'attention avec hyperactivité (TDAH), nécessitant la prise quotidienne de Ritalin ${ }^{\circledR}$. Selon Juneau et Boucher (2004), ces enfants sont davantage portés que les autres à faire preuve de violence. De plus, près du tiers d'entre eux éprouvent divers problèmes de santé physique ou mentale nécessitant une médication prescrite par un professionnel de la santé. Par ailleurs, la moitié des enfants de l'échantillon bénéficient des services d'un travailleur social tandis que deux sur cinq font l'objet d'un suivi auprès d'un psychologue. Également, trois garçons voient régulièrement un pédopsychiatre ou un pédiatre. Finalement, trois enfants participent à des programmes de rééducation du langage (orthothérapie) et un garçon bénéficie de services de rééducation physique (ergothérapie). Les antécédents médicaux des 3 à 9 ans sont présentés au tableau 3.12.

\subsection{LES LIMITES DE LA RECHERCHE}

Dans la présente recherche, nous avons identifié plusieurs limites. La première concerne le nombre restreint de sujets y ayant participé, qui ne permet pas la généralisation, mais la transférabilité des résultats obtenus à l'ensemble des parents d'enfants ayant les mêmes caractéristiques qui fréquentent une école élémentaire de langue française en Ontario. La deuxième limite vient du fait que les résultats de la présente recherche traduisent uniquement les perceptions des parents y ayant participé. 
TABleau 3.12

Les antécédents médicaux des 3 à 9 ans $(n=60)$

\begin{tabular}{lcccc}
\hline & \multicolumn{2}{c}{ Garçons } & \multicolumn{2}{c}{ Filles } \\
\cline { 2 - 5 } & $N$ & $\%$ & $N$ & $\%$ \\
\cline { 2 - 5 } & 54 & 90,0 & 6 & 10,0 \\
\hline Suivi par un travailleur social & 26 & 43,0 & 4 & 6,5 \\
\hline Suivi par un psychologue & 22 & 36,0 & 2 & 3,5 \\
\hline $\begin{array}{l}\text { Médicament prescrit par un } \\
\text { professionnel de la santé }\end{array}$ & 16 & 27,0 & 2 & 3,5 \\
\hline $\begin{array}{l}\text { Problèmes de santé physique } \\
\text { ou mentale }\end{array}$ & 14 & 24,0 & 2 & 3,5 \\
\hline $\begin{array}{l}\text { Trouble du déficit de l'attention } \\
\text { avec hyperactivité }\end{array}$ & 14 & 24,0 & 1 & 1,5 \\
\hline Ritalin ${ }^{\circledR}$ & 12 & 20,0 & 1 & 1,5 \\
\hline $\begin{array}{l}\text { Suivi par un pédopsychiatre } \\
\text { Suivi par un pédiatre }\end{array}$ & 3 & 5,0 & & \\
\hline $\begin{array}{l}\text { Suivi en rééducation du langage } \\
\text { (orthothérapie) }\end{array}$ & 1 & 5,0 & & \\
\hline $\begin{array}{l}\text { Suivi en rééducation physique } \\
\text { (ergothérapie) }\end{array}$ & 1 & 1,5 & 2 & 3,5 \\
\hline
\end{tabular}

Comme ces perceptions n'ont fait l'objet ni d'une consultation, ni d'une validation auprès du personnel œuvrant dans le milieu scolaire, dans les services sociaux ou communautaires, elles se limitent aux sujets constituant l'échantillon. La troisième limite concerne le fait que les filles et les pères ne sont pas assez représentés dans l'échantillon comparativement aux garçons et aux mères, malgré tous les efforts déployés pour en recruter davantage. La quatrième limite a trait aux familles vivant en contexte de pauvreté, qui sont assez peu représentées dans l'échantillon. Celui-ci ne regroupe en effet que 18 familles vivant dans un tel contexte, dont seulement six parents d'enfants de 3 à 6 ans. Enfin, la cinquième limite concerne les familles vivant en contexte minoritaire pluriethnique, qui ne sont pas suffisamment nombreuses dans l'échantillon pour refléter la réalité propre à la communauté francophone de l'Ontario. À ce sujet, seulement sept familles vivant dans un tel contexte ont accepté de participer à la présente recherche.

Malgré ces limites, l'échantillon se compose uniquement de parents dont les enfants ont des comportements violents au préscolaire et au primaire, respectant ainsi un principe d'homogénéité. En réponse à de tels comportements, ces enfants ont fait l'objet d'une suspension ou d'une 
possibilité de suspension scolaire, au sens de la Loi sur la sécurité dans les écoles (Ministère de l'Éducation de l'Ontario, 2000) et du Code de conduite provincial (Ministère de l'Éducation de l'Ontario, 2001a). En participant à la présente recherche, ces familles ont accepté de parler de leur vécu, de leur vision et de leur réalité quotidienne, tout en faisant part de leurs préoccupations et des solutions qu'ils envisagent. En ce sens, les résultats obtenus nous permettent d'amorcer un processus de réflexion et d'intervention auprès d'enfants ayant des comportements violents au préscolaire et au primaire. Un tel processus pourra ultérieurement s'appliquer à d'autres communautés francophones, incluant celles où la langue française est minoritaire au Canada.

\section{LES COMPORTEMENTS VIOLENTS DES 3 À 6 ANS}

Dans cette partie, nous abordons le passage entre la maison, la garderie et le cycle préscolaire. Puis, nous présentons les comportements violents, les problèmes internalisés et externalisés, les problèmes de socialisation, la durée, la fréquence, le lieu et le moment des comportements violents, de même que la trajectoire qu'ils empruntent. En terminant, nous présentons les pratiques éducatives parentales ayant un effet positif et négatif, les stresseurs familiaux affectant négativement les comportements et les facteurs explicatifs de la situation-problème de l'enfant à l'école selon les perceptions des parents de ce sous-échantillon.

\subsection{LE PASSAGE DE LA MAISON OU DE LA GARDERIE AU PRÉSCOLAIRE}

Le passage de la maison ou de la garderie au préscolaire n'est pas sans occasionner de grands bouleversements dans la vie de l'enfant. D'un système familial où il ne transige qu'avec des règles établies avec l'environnement immédiat, soit avec ses parents et la fratrie, et ce, généralement depuis sa naissance, l'enfant qui entre à la garderie se voit confronté avec les premières règles de fonctionnement institutionnelles. Ces règles concordent parfois avec celles qui émanent du milieu familial ; à l'inverse, elles peuvent aussi se situer en rupture avec le vécu de l'enfant, ce qui exige de lui une période intense d'ajustements afin de répondre à la demande d'adaptation au nouveau milieu. La nature des premières relations que l'enfant entretient avec les autres jette les bases sur lesquelles s'édifiera sa socialisation. L'une des transformations majeures qui s'opère dans la vie de l'enfant est l'arrivée des pairs comme agents de socialisation. L'entrée 
au préscolaire signifie que les enfants deviennent progressivement moins dépendants de leurs parents, tandis que s'accroît parallèlement l'influence des pairs sur leurs comportements (Fortin, 1999).

\subsection{LES COMPORTEMENTS VIOLENTS}

Les comportements violents des enfants de ce sous-échantillon ont été divisés en deux catégories, inspirées de la Conflict Tactics Scale de Straus et Gelles (1990). La première regroupe la violence physique, c'est-à-dire les comportements qui portent atteinte physiquement et qui blessent la personne visée. La deuxième regroupe deux sous-catégories, soit la violence verbale sans geste, qui consiste à insulter, menacer de violence physique, etc., et la violence verbale avec gestes, soit le fait de lancer des objets, de frapper des objets, de taper du pied, etc. La violence verbale comprend également des gestes de violence symbolique, à savoir de bouder, de refuser de discuter, etc.

\subsubsection{La violence physique}

Chez les 23 enfants de 3 à 6 ans, la violence physique ressort des données d'entrevue. La majorité des garçons de ce sous-échantillon frappent, tapent et donnent des coups de pied. Un peu moins des deux tiers des enfants donnent des coups de poing, mordent, poussent, se battent, malmènent ou intimident leurs pairs. Les parents décrivent certains comportements violents remarqués chez leur enfant: Au début de l'année, c'était à coups de poing. Maintenant ça semble être mieux, il va juste pousser $(91)^{*}$; S'il n'aime pas ce que les autres disent, il va frapper (92); Il avait donné un coup de pied à son frère qui était en train de jouer, et à l'estomac en plus! (108).

Le plus souvent, les jeunes enfants vont surtout frapper les pairs (Cowie, 2000) ou les frères et sœurs. Ces enfants vont rarement diriger leur violence contre un adulte. En effet, un très petit nombre d'enfants ont frappé leur enseignant. Quoique le nombre d'enfants qui frappent leurs parents soit légèrement plus élevé, il demeure toutefois assez minime par rapport à l'ensemble de ce sous-échantillon. Également, une petite fille a coupé les cheveux d'un autre enfant avec des ciseaux, en passant dangereusement près des yeux. Les comportements de violence physique des 3 à 6 ans sont présentés au tableau 3.13 .

* Le chiffre entre parenthèses correspond au numéro du répondant. 
TABLEAU 3.13

Les comportements de violence physique des 3 à 6 ans $(n=23)$

\begin{tabular}{lcccc}
\hline & \multicolumn{2}{c}{ Garçons } & \multicolumn{2}{c}{ Filles } \\
\cline { 2 - 5 } & $N$ & $\%$ & $N$ & $\%$ \\
\cline { 2 - 5 } & 20 & 87,0 & 3 & 13,0 \\
\hline Frappent et tapent & 20 & 87,0 & 1 & 4,0 \\
\hline Donnent des coups de pied & 20 & 87,0 & 1 & 4,0 \\
\hline Donnent des coups de poing & 14 & 61,0 & 1 & 4,0 \\
\hline Mordent & 13 & 56,0 & 1 & 4,0 \\
\hline Se battent & 13 & 56,0 & 1 & 4,0 \\
\hline Poussent & 11 & 48,0 & 2 & 8,0 \\
\hline Malmènent ou intimident & 11 & 48,0 & 1 & 4,0 \\
\hline Frappent l'enseignant & 5 & 22,0 & & \\
\hline Frappent les parents & 3 & 13,0 & & \\
\hline Coupe les cheveux & & & 1 & 4,0 \\
\hline
\end{tabular}

\subsubsection{La violence verbale avec ou sans gestes}

Près des trois quarts des enfants de notre échantillon détruisent leurs jouets, ceux des autres ou même d'autres objets qui leur tombent sous la main. Les deux tiers d'entre eux frappent des objets, les lancent comme des projectiles, ou même leur donnent des coups de pied.

Quant à la violence verbale sans geste, un peu plus de la moitié des enfants insultent les autres, plus de deux enfants sur cinq font des menaces et les trois quarts d'entre eux blâment les autres. Également, le quart des enfants utilisent un langage vulgaire pouvant porter atteinte à l'intégrité des personnes visées. Les comportements de violence verbale, avec ou sans gestes, des 3 à 6 ans sont présentés au tableau 3.14. 
TABLEAU 3.14

Les comportements de violence verbale avec ou sans gestes des 3 à 6 ans $(n=23)$

\begin{tabular}{lcccc}
\hline & \multicolumn{2}{c}{ Garçons } & \multicolumn{2}{c}{ Filles } \\
\cline { 2 - 5 } & $\boldsymbol{N}$ & $\%$ & $\boldsymbol{N}$ & $\%$ \\
\cline { 2 - 5 } & 20 & 87,0 & 3 & 13,0 \\
\hline Détruisent des objets & 15 & 65,0 & 2 & 8,0 \\
\hline Frappent des objets & 14 & 61,0 & 1 & 4,0 \\
\hline Lancent des objets & 13 & 56,0 & 2 & 8,0 \\
\hline Donnent des coups de pied aux objets & 13 & 56,0 & 1 & 4,0 \\
\hline Insultent & 11 & 48,0 & 2 & 8,0 \\
\hline Font des menaces & 9 & 39,0 & 1 & 4,0 \\
\hline Blâment & 7 & 30,0 & 2 & 8,0 \\
\hline Utilisent un langage vulgaire & 5 & 22,0 & 1 & 4,0 \\
\hline
\end{tabular}

\subsubsection{Les problèmes internalisés}

L'anxiété, l'insécurité et les sentiments dépressifs ressortent chez les trois quarts des enfants de ce sous-échantillon aux prises avec des problèmes internalisés. Les résultats de la présente recherche se différencient de ceux des écrits en raison de l'accent mis par les parents sur la souffrance morale vécue par leur enfant: Il est dans un stade où il est tout le temps triste (92); Il se sent triste parce qu'il ne se sent pas à la hauteur de ce qu'on lui demande (99).

Près des deux tiers des enfants manquent d'estime de soi et de confiance en soi. Également, quelques-uns s'isolent et se blâment: Il se disait méchant et se blâmait pour tout (109); Être dans son petit coin (110).

Un dernier entretient des idées suicidaires: Il me demande souvent s'il va mourir. Il dit qu'il ne veut pas mourir (92). Les problèmes internalisés des 3 à 6 ans sont présentés au tableau 3.15. 
TABLEAU 3.15

Les problèmes internalisés des 3 à 6 ans $(n=23)$

\begin{tabular}{lcccc}
\hline & \multicolumn{2}{c}{ Garçons } & \multicolumn{2}{c}{ Filles } \\
\cline { 2 - 5 } & $\boldsymbol{N}$ & $\%$ & $\boldsymbol{N}$ & $\%$ \\
\cline { 2 - 5 } & 20 & 87,0 & 3 & 13,0 \\
\hline Éprouvent de l'anxiété & 15 & 65,0 & 2 & 8,0 \\
\hline Se sentent insécures & 15 & 65,0 & 1 & 4,0 \\
\hline Ont des sentiments dépressifs & 14 & 61,0 & 1 & 4,0 \\
\hline Manquent d'estime de soi & 13 & 56,0 & 2 & 8,0 \\
\hline Manquent de confiance en soi & 11 & 48,0 & 2 & 8,0 \\
\hline S'isolent & 5 & 25,0 & 2 & 8,0 \\
\hline Se blâment & 2 & 8,0 & 1 & 4,0 \\
\hline Entretient des idées suicidaires & 1 & 4,0 & & \\
\hline
\end{tabular}

\subsubsection{Les problèmes externalisés}

Les enfants de ce sous-échantillon sont également aux prises avec nombre de problèmes externalisés: Avoir quelqu'un dans son espace qui le regarde, il «réactionne» [réagit]! (108).

Presque tous les parents déclarent que leur enfant n'écoute pas les consignes. Les trois quarts d'entre eux ont tendance à s'opposer à l'adulte, tout en faisant preuve de suractivité: Il est superactif, il ne peut pas rester calme, comme s'il ne peut pas rester assis (102).

Ces dernières caractéristiques sont souvent associées aux enfants ayant le Trouble du déficit de l'attention avec hyperactivité (TDAH). Selon Juneau et Boucher (2004), il est parfois difficile de départager ce qui revient à ce diagnostic et à la problématique des comportements violents. Cette situation complexifie la situation-problème de l'enfant, en laissant facilement place à des interprétations hâtives de la part du personnel scolaire.

D'autres comportements d'opposition sont également très présents chez les deux tiers des enfants, tels que chialer ou faire des crises. Également, on constate des sautes d'humeur et de l'impulsivité chez plus de la moitié d'entre eux, tandis que les colères, la frustration et l'impatience sont présents chez le quart des enfants. Les problèmes externalisés des 3 à 6 ans sont présentés au tableau 3.16. 
TABleau 3.16

Les problèmes externalisés des 3 à 6 ans $(n=23)$

\begin{tabular}{lcccc}
\hline & \multicolumn{2}{c}{ Garçons } & \multicolumn{2}{c}{ Filles } \\
\cline { 2 - 5 } & $\boldsymbol{N}$ & $\%$ & $\boldsymbol{N}$ & $\%$ \\
\cline { 2 - 5 } & 20 & 87,0 & 3 & 13,0 \\
\hline N'écoutent pas les consignes & 18 & 78,0 & 3 & 13,0 \\
\hline S'opposent à l'adulte & 15 & 65,0 & 2 & 8,0 \\
\hline Font preuve de suractivité & 15 & 65,0 & 1 & 4,0 \\
\hline Chialent & 14 & 61,0 & 2 & 8,0 \\
\hline Font des crises & 13 & 56,0 & 2 & 8,0 \\
\hline Ont des sautes d'humeur & 13 & 56,0 & 2 & 8,0 \\
\hline Font preuve d'impulsivité & 11 & 48,0 & 2 & 8,0 \\
\hline Font des colères & 7 & 30,5 & 1 & 4,0 \\
\hline Éprouvent de la frustration & 6 & 26,0 & 1 & 4,0 \\
\hline Font preuve d'impatience & 5 & 22,0 & 1 & 4,0 \\
\hline
\end{tabular}

\subsubsection{Les problèmes de socialisation}

La majorité des enfants de ce sous-échantillon qui éprouvent des problèmes de socialisation ont de grandes difficultés à fonctionner en groupe. Plus des trois quarts d'entre eux s'isolent et jouent plus souvent seuls: Il ne voit plus personne. Il n'a pas d'amis (80). Or, selon les parents, ces enfants aiment ou préfèrent jouer en groupe. Afin de se faire des amis, ils utilisent diverses stratégies: Il a des bonbons dans les mains. Puis, il va arriver devant un petit ami et va dire: Écoute, j'ai des bonbons, si tu es mon ami, je vais t'en donner (99).

À l'inverse, chez les trois quarts des enfants, la difficulté de partager avec les pairs constitue une barrière lorsqu'ils cherchent à se faire des amis. Par ailleurs, plus de la moitié d'entre eux refusent souvent de se plier aux règles. Finalement, un peu plus du tiers des parents déclarent avoir appris que certains pairs se moquent de leur enfant, le poussent ou le frappent.

Dans la foulée des écrits consultés, les enfants violents deviennent facilement la cible de rejet de la part de leurs pairs. Ils peuvent également devenir davantage violents lorsqu'ils sont eux-mêmes victimes de la violence exercée par leurs pairs. Ce lien entre violence et victimisation s'avère très fréquent, créant ainsi un cercle vicieux difficile à briser. Les problèmes de socialisation des 3 à 6 ans sont présentés au tableau 3.17. 
TABLEAU 3.17

Les problèmes de socialisation des 3 à 6 ans $(n=23)$

\begin{tabular}{lcccc}
\hline & \multicolumn{2}{c}{ Garçons } & \multicolumn{2}{c}{ Filles } \\
\cline { 2 - 5 } & $N$ & $\%$ & $N$ & $\%$ \\
\cline { 2 - 5 } & 20 & 87,0 & 3 & 13,0 \\
\hline Ne fonctionnent pas en groupe & 19 & 82,5 & 3 & 13,0 \\
\hline S'isolent & 18 & 78,0 & 1 & 4,0 \\
\hline Jouent seuls & 18 & 78,0 & 1 & 4,0 \\
\hline Ont de la difficulté à partager & 17 & 74,0 & 2 & 8,0 \\
\hline Refusent de se plier aux règles & 11 & 48,0 & 2 & 8,0 \\
\hline Sont l'objet de moqueries & 8 & 35,0 & 1 & 4,0 \\
\hline Se font pousser par les autres & 7 & 31,0 & 1 & 4,0 \\
\hline Se font frapper par les autres & 6 & 26,0 & 1 & 4,0 \\
\hline
\end{tabular}

\subsection{LA DURÉE ET LA FRÉQUENCE DES COMPORTEMENTS VIOLENTS}

Pour être considérés problématiques, les comportements violents doivent s'avérer fréquents et stables dans le temps (Cowie, 2000; Day, Golench, MacDougall et Beals-Gonzales, 1995; Tremblay, 2000). À ce sujet, Loeber et Farrington (2000) sont d'avis qu'ils doivent être observés durant une période de six mois ou plus.

Sans pouvoir fixer précisément depuis combien de temps ils durent, quelques parents affirment que les comportements violents de leur enfant sont présents depuis une assez longue période: Depuis que l'enfant a 2 ans, j'ai eu plein de problèmes avec lui (51); Depuis un an et demi, j'ai remarqué une différence. Une certaine intensité (111).

Quant à la fréquence des comportements violents, elle peut varier considérablement d'un enfant à l'autre, allant d'une fois par jour à une fois par mois. À ce sujet, les parents observent que chez le quart des enfants de ce sous-échantillon, ils se produisent à raison d'une fois par jour. Chez un autre quart, ces comportements sont présents une fois par semaine, tandis que chez un autre quart, ils se produisent plusieurs fois par semaine. Enfin, chez le dernier quart, ils sont présents soit plusieurs fois par mois ou une seule fois par mois. Également, chez une dernière petite fille, les comportements violents se présentent de manière épisodique. La fréquence des comportements violents des 3 à 6 ans est présentée au tableau 3.18. 
Tableau 3.18

La fréquence des comportements violents des 3 à 6 ans $(n=23)$

\begin{tabular}{lrrrr}
\hline & \multicolumn{2}{c}{ Garçons } & \multicolumn{2}{c}{ Filles } \\
\cline { 2 - 5 } & $\boldsymbol{N}$ & $\%$ & $\boldsymbol{N}$ & $\%$ \\
\cline { 2 - 5 } & 20 & 87,0 & 3 & 13,0 \\
\hline Une fois par jour & 6 & 26,0 & & \\
\hline Plusieurs fois par semaine & 6 & 26,0 & 1 & 4,0 \\
\hline Une fois par semaine & 6 & 26,0 & & \\
\hline Plusieurs fois par mois & 1 & 4,0 & 1 & 4,0 \\
\hline Une fois par mois & 1 & 4,0 & & \\
\hline De manière épisodique & & & 1 & 4,0 \\
\hline
\end{tabular}

\subsection{LE LIEU ET LE MOMENT DES COMPORTEMENTS VIOLENTS}

Pour ce qui est du lieu où se produisent les comportements violents, la majorité des parents déclarent que leur enfant ne fait preuve de tels comportements qu'à l'école. À l'inverse, deux parents de ce sous-échantillon rapportent que leur enfant se comporte mieux à l'école qu'à la maison.

En ce qui a trait au moment où se produisent les comportements violents, le tiers des parents mentionnent que c'est lorsque la fatigue est plus grande chez leur enfant, soit vers la fin de l'après-midi. À ce sujet, une mère est catégorique: C'est pire quand il est fatigué. Après 5 heures, «forget it»! (108).

Ainsi, il devient important de considérer non seulement le rôle que les pairs jouent au préscolaire par rapport à l'émergence des comportements violents, mais également le moment de la journée, voire de l'année où ils surviennent, tant à l'école qu'à la maison. Le lieu et le moment des comportements violents des 3 à 6 ans sont présentés au tableau 3.19.

Tableau 3.19

Le lieu et le moment des comportements violents des 3 à 6 ans $(n=23)$

\begin{tabular}{|c|c|c|c|c|}
\hline & \multicolumn{2}{|c|}{ Garçons } & \multicolumn{2}{|c|}{ Filles } \\
\hline & $N$ & $\%$ & $N$ & $\%$ \\
\hline & 20 & 87,0 & 3 & 13,0 \\
\hline À l'école & 19 & 82,5 & 2 & 8,0 \\
\hline$\overline{\mathrm{A}}$ la fin de l'après-midi & 6 & 26,0 & 2 & 8,0 \\
\hline À la maison & 1 & 4,0 & 1 & 4,0 \\
\hline
\end{tabular}




\subsection{LA TRAJECTOIRE DES COMPORTEMENTS VIOLENTS}

Selon la moitié des parents, les difficultés de leur enfant ont commencé avec l'entrée à la maternelle ou au jardin. Pour un peu moins d'un tiers des parents, elles étaient présentes même avant, soit avec l'entrée à la garderie. À ce sujet, près du quart des parents se contredisent tout au long de leur entrevue et déclarent deux possibilités: Il a toujours été comme ça [...]. Ça fait... Bien, depuis l'année passée, depuis qu'il va à la garderie (102).

Comme le suggère Tremblay (2000), il est possible que ces parents perçoivent la violence de leur enfant comme étant assez récente en raison de ses manifestations qui ont évolué avec le temps, les étapes de son développement et l'ampleur des contacts avec les pairs. Ils oublient facilement des événements lointains à l'occasion desquels des comportements violents pouvaient être présents.

En général, les parents de ce sous-échantillon mentionnent des événements survenus au cours de l'année et demie ayant précédé l'entrevue de recherche. Ainsi, quelques parents déclarent que les comportements violents de leur enfant étaient présents bien avant l'entrée au préscolaire. Par ailleurs, un peu plus du tiers d'entre eux déclarent que les comportements de leur enfant sont pires depuis le début du préscolaire. La trajectoire des comportements violents des 3 à 6 ans est présentée au tableau 3.20.

TABLEAu 3.20

La trajectoire des comportements violents des 3 à 6 ans $(n=23)$

\begin{tabular}{lrrrr}
\hline & \multicolumn{2}{c}{ Garçons } & \multicolumn{2}{c}{ Filles } \\
\cline { 2 - 5 } & $\boldsymbol{N}$ & $\%$ & $\boldsymbol{N}$ & $\%$ \\
\cline { 2 - 5 } & 20 & 87,0 & 3 & 13,0 \\
\hline $\begin{array}{l}\text { Avec l'entrée à la maternelle/ } \\
\text { au jardin }\end{array}$ & 11 & 48,0 & 1 & 4,0 \\
\hline Avec l'entrée à la garderie & 6 & 26,0 & 1 & 4,0 \\
\hline Avant l'entrée au préscolaire & 3 & 13,0 & 1 & 4,0 \\
\hline Pires depuis le préscolaire & 8 & 35,0 & 1 & 4,0 \\
\hline
\end{tabular}




\subsection{Les PRATIQUES ÉdUCATIVES PARENTALES}

Dans les ouvrages consultés, une des raisons importantes justifiant les comportements violents chez les enfants semble être la difficulté qu'ont leurs parents de leur imposer une discipline et de maintenir une routine (Akande, 2001; Vézina, Bradet, Lord, Pelletier et Thibault, 1995; Armstrong, Caron et Guimond, 1992). Pourtant, les parents de ce sous-échantillon déclarent offrir un éventail de pratiques éducatives appropriées, tel que recommandé par les professionnels et enseigné dans les groupes de parentage.

L'inconstance dans l'utilisation des pratiques éducatives ayant un effet positif et négatif, comme la décrivent les parents, peut être associée au phénomène de la désirabilité sociale ainsi qu'au sentiment de culpabilité qu'ils éprouvent souvent en regard des comportements violents de leur enfant. Par ailleurs, les deux tiers des parents se déclarent satisfaits de leurs pratiques éducatives.

Comme l'avancent Trudelle et Montambault (1994), ces parents présentent généralement un important sentiment de compétence par rapport à leurs pratiques éducatives réelles. Ce sentiment peut également être attribuable au fait que tous les enfants ou leur famille, à l'exception d'une, bénéficient de services de relation d'aide. Le fait de recevoir un suivi de la part d'un professionnel peut expliquer en partie pourquoi les parents éprouvent un fort sentiment de compétence éducative même si leur enfant éprouve des difficultés comportementales persistantes, pour lesquelles ils utilisent des pratiques ayant parfois un effet parfois positif, parfois négatif.

\subsubsection{Les pratiques ayant un effet positif}

Parmi les pratiques éducatives parentales ayant un effet positif chez l'enfant, près des trois quarts des parents utilisent le retrait et le renforcement positif, soit des compliments, des sorties ou des cadeaux. Un peu plus de la moitié d'entre eux ont recours à la perte de privilèges, à la technique 1-2-3 (avertissement-arrêt d'agir-conséquence) ou au dialogue avec leur enfant en lui expliquant ce qui est acceptable comme comportement. Par ailleurs, les deux tiers des parents déclarent utiliser toujours la même technique et deux sur cinq déclarent maintenir une routine que l'enfant doit suivre. Les pratiques éducatives parentales ayant un effet positif chez les 3 à 6 ans sont présentées au tableau 3.21. 
TABLEAU 3.21

Les pratiques éducatives parentales ayant un effet positif chez les 3 à 6 ans $(n=23)$

\begin{tabular}{lcccc}
\hline & \multicolumn{2}{c}{ Garçons } & \multicolumn{2}{c}{ Filles } \\
\cline { 2 - 5 } & $\boldsymbol{N}$ & $\%$ & $\boldsymbol{N}$ & $\%$ \\
\cline { 2 - 5 } & 20 & 87,0 & 3 & 13,0 \\
\hline Retrait & 14 & 61,0 & 3 & 13,0 \\
\hline Renforcement positif & 14 & 61,0 & 2 & 8,0 \\
\hline Toujours la même technique & 13 & 56,0 & 2 & 8,0 \\
\hline Dialogue & 12 & 52,0 & 2 & 8,0 \\
\hline Perte de privilèges & 11 & 48,0 & 2 & 8,0 \\
\hline Technique 1-2-3 & 11 & 48,0 & 2 & 8,0 \\
\hline Maintien d'une routine & 8 & 35,0 & 1 & 4,0 \\
\hline
\end{tabular}

\subsubsection{Les pratiques ayant un effet négatif}

Certains parents jugent qu'ils utilisent des pratiques éducatives parentales ayant un effet négatif chez leur enfant. La moitié d'entre eux affirment qu'ils n'interviennent pas toujours ou, à l'inverse, qu'ils interviennent trop, surtout lorsqu'ils sont fatigués. Deux parents sur cinq déclarent qu'ils recourent à des pratiques coercitives, comme crier après leur enfant: J'essaie d'être patiente, mais il y a des jours où j'en ai vraiment pas. Je frappe pas mes enfants. Mais des fois, ma voix est un peu forte. Puis, j'ai tendance à crier pas mal (110).

Bien qu'ils avouent y recourir, ces parents prennent conscience que les pratiques coercitives ont un effet négatif chez leur enfant. C'est pourquoi ils essaient, dans la mesure du possible, de ne plus le faire: Crier le fige, sauf qu'il ne comprend pas pourquoi je le fais (44); Quand on élève la voix, c'est pire. Plus on s'énerve, plus il s'énerve (51).

Le tiers des parents ont tendance à punir leur enfant de manière abusive, ce qui contribue à détériorer le climat familial: Pendant une couple de mois, à chaque soir pendant une heure et demie de temps. Il sortait de sa chambre, je le reconduisais. J'étais "écourée net» [j'en avais marre]! (111).

Un parent sur cinq admet qu'il frappe ou tape parfois son enfant et, comme dans le cas des cris, il déclare ne plus vouloir le faire: Taper, mais j'aime pas ça. Je ne veux plus le faire (09). 
Deux d'entre eux soulèvent le fait de ne pas faire preuve d'une discipline assez stricte. Un autre confie que son enfant est trop gâté (99), tandis qu'un dernier avoue avoir un grand manque de structure (108). Les pratiques éducatives parentales ayant un effet négatif chez les 3 à 6 ans sont présentées au tableau 3.22.

TABLEAU 3.22

Les pratiques éducatives parentales ayant un effet négatif
chez les 3 à 6 ans $(n=23)$

\begin{tabular}{lrrrr}
\hline & \multicolumn{2}{c}{ Garçons } & \multicolumn{2}{c}{ Filles } \\
\cline { 2 - 5 } & $\boldsymbol{N}$ & $\%$ & $\boldsymbol{N}$ & $\%$ \\
\cline { 2 - 5 } & 20 & 87,0 & 3 & 13,0 \\
\hline N'interviennent pas toujours & 10 & 43,0 & 2 & 8,0 \\
\hline Interviennent trop & 9 & 39,0 & 1 & 4,0 \\
\hline Crient & 8 & 35,0 & 2 & 8,0 \\
\hline Punissent de manière abusive & 7 & 31,0 & 1 & 4,0 \\
\hline Frappent et tapent & 3 & 13,0 & 1 & 4,0 \\
\hline N'ont pas de discipline assez stricte & 2 & 8,0 & & \\
\hline Gâte & 1 & 4,0 & & \\
\hline Manque de structure & 1 & 4,0 & & \\
\hline
\end{tabular}

\subsection{LES STRESSEURS FAMILIAUX}

Les comportements violents de l'enfant affectent négativement la situation familiale (Cowie, 2000 ; Turcotte, St-Amand, Beaudoin et Champoux, 2001). Certains parents croient que l'inverse est également vrai, à savoir que ce sont les événements stressants de la vie familiale qui ont un effet négatif sur leurs comportements, tant et si bien qu'il devient difficile, voire impossible de déterminer lequel vient avant l'autre.

Quoi qu'il en soit, les stresseurs familiaux jouent un rôle indéniable dans l'émergence des comportements violents au préscolaire et au primaire. À ce sujet, les parents de ce sous-échantillon énumèrent différents événements stressants vécus par la famille qui leur semblent avoir un effet négatif sur les comportements de leur enfant: J'ai comme l'impression qu'ils vivent les mêmes rythmes que nous autres. Cet enfant-là a le SPM [Syndrome prémenstruel] en même temps que moi (99). 
Plus spécifiquement, cinq différents stresseurs familiaux sont identifiés selon un ordre décroissant d'importance, soit les conflits intrafamiliaux, le peu ou l'absence de relations avec le père, le manque de temps des parents, l'isolement social et la maladie de la mère.

\subsubsection{Les conflits intrafamiliaux}

Les conflits intrafamiliaux représentent une importante source de stress au sein de la famille. Ils incluent les disputes, la séparation ou le divorce et la violence conjugale. À cet égard, les deux tiers des parents mentionnent vivre ou avoir vécu de tels conflits et le tiers d'entre eux sont persuadés qu'ils constituent la cause principale des comportements violents de leur enfant. Une des mères explique que le stress découlant de tels conflits affecte grandement le climat familial: Quand le niveau de stress est extrêmement élevé, il y a des problèmes partout. Le niveau de stress est gardé de façon normale, il n'y en a pas (109).

Les disputes ont mené, dans deux cas, à la séparation ou au divorce. Ces parents exposent la manière dont cette situation bouleverse et affecte négativement les comportements de leur enfant: Mon fils me dit: Papa, j'ai un trou dans mon cour. Maman a fait beaucoup de peine à toi! [...]. C'est comme une flèche que j'ai dans mon cour [...]. Quand cette flèche-là, je la sens, je deviens un enfant méchant, c'est pour ça que je fais mal aux autres (93).

Ces parents reconnaissent que la séparation ou le divorce est la cause principale des comportements violents de leur enfant. En effet, les situations qui y mènent engendrent une qualité moindre du fonctionnement familial et fragilisent l'enfant (Flanagan, 1999; Saint-Jacques, Drapeau et Cloutier, 2001). En ce sens, la séparation ou le divorce des parents semblent être de loin le stresseur familial ayant le plus d'effets négatifs sur les problèmes comportementaux des enfants (Jutras, 1999; Saint-Jacques, Drapeau et Cloutier, 2001; Schick, 2002).

Un dernier type de conflit abordé par les parents concerne la violence conjugale. Trois mères croient qu'elle a eu un effet très négatif sur les comportements de leur enfant. L'une d'elles, qui a subi de la part de son ex-mari des sévices physiques dont son jeune fils a été témoin, se confie: J'ai été une femme battue et mon fils a vu ça à l'âge de 4 ans. Il a vu "you do what you want». Depuis ce temps-là, il est méchant. Si ça ne va pas à sa façon, il frappe. Il dit qu'il a pas peur de moi (80).

Lorsqu' on étudie la problématique des comportements violents, il ne faut pas minimiser celle des enfants témoins de violence conjugale, car elle complexifie la situation-problème (Bourassa et Turcotte, 1998). 


\subsubsection{Le peu ou l'absence de relations avec le père}

L'Institut Vanier de la famille (2000) considère que les pères d'aujourd'hui accordent en moyenne plus de temps à leurs enfants qu'il y a dix ans. À l'inverse, la séparation ou le divorce entraînent une diminution significative, voire une rupture complète des relations entre le père et ses enfants (Cavell, 2000). La diminution ou l'absence de relations mène parfois le père au désengagement (Quéniart, 2001).

La moitié des familles de ce sous-échantillon sont monoparentales, c'est-à-dire qu'elles sont dirigées par une mère seule et, dans un seul cas, par un père seul. Dans le cas des familles dirigées par une mère seule, les deux tiers des enfants sont peu en contact avec le père biologique tandis que le tiers d'entre eux ne le voient jamais. Trois mères parmi ces dernières familles monoparentales voient dans l'absence du père la cause principale des comportements violents de leur enfant. Aucune de ces quatre mères ne souligne les effets négatifs que cette absence génère dans sa propre vie, mais elles insistent sur ceux que cette situation occasionne dans la vie de leur enfant: Il a une absence de modèle masculin, car je vis seule. Il voit son père aux deux semaines. Des fois, il ne le prend pas (01); Il ne voit pas son père et dans son environnement, ce ne sont que des femmes. La présence de son père lui manque beaucoup (28).

Environ la moitié des mères de ce sous-échantillon indiquent que leur enfant a très peu de relations avec le père. Cette situation est également mentionnée par une mère provenant d'une famille intacte. Cette mère observe que son conjoint interagit peu avec leur enfant à cause de ses longues heures de travail, ce qui semble lui nuire. Comme les mères le formulent dans leurs commentaires, la présence du père est importante pour le développement de l'enfant (Flanagan, 1999; Nugent, 1992). Son absence ou le peu de relations avec lui peut perturber l'enfant et avoir un effet négatif sur ses comportements.

\subsubsection{Le manque de temps des parents}

Plus de la moitié des parents de ce sous-échantillon soutiennent qu'ils aimeraient passer en moyenne plus de temps avec leur enfant: Il faut que je m'organise pour avoir plus de temps, et alors, ça va bien aller (111).

Huit d'entre eux indiquent qu'ils éprouvent des difficultés à concilier le travail et la famille, en raison d'un horaire surchargé, de lourdes responsabilités et du surcroît de stress qui en découle. Sur ce nombre, quatre croient que le manque de temps accordé à leur enfant constitue la cause principale de ses comportements violents: À la maison, nous sommes deux parents qui travaillent, qui travaillent très très fort pour répondre aux besoins des 
enfants, et puis qui donnent tout ce qu'ils peuvent. Naturellement, c'est sûr que par bouts, c'est épuisant. Autant pour les parents que pour les enfants. Travailler au-dessus de 40 heures par semaine, chacun de notre côté. De partir de la maison à 7 heures 15 et de revenir à 6 heures 15. Là, c'est le souper, les devoirs de l'aîné, les bains, puis le dodo. On a pas de temps pour s'amuser avec eux. Il faut aller aux priorités (99).

Toutes les familles biparentales sauf une et toutes les familles monoparentales sauf deux de ce sous-échantillon sont sur le marché du travail. Deux études récentes, l'une menée par Santé Canada et intitulée Enquête nationale sur le conflit entre le travail et la vie personnelle (Higgins et Duxbury, 2002), l'autre menée par Développement des ressources humaines du Canada et intitulée Témoignages canadiens: à la recherche de la conciliation travail-vie personnelle (Duxbury, Higgins et Coghill, 2003), confirment qu'il y a une augmentation globale du temps de travail chez les Canadiens. En moyenne, ceux-ci travaillent 42,5 heures par semaine, tandis que pour le quart d'entre eux, il est question de plus de 50 heures. Cette situation amène une réduction d'un tiers du temps disponible consacré aux enfants (Higgins et Duxbury, 2002). Puisque les parents doivent travailler de longues heures, il ne reste que très peu de temps pour réaliser toutes les tâches familiales (Higgins et Duxbury, 2002; Institut Vanier, 2000 ; McCain et Mustard, 1999).

Les parents de ce sous-échantillon étant presque tous engagés sur le marché du travail, ils peuvent facilement vivre des horaires analogues à ceux auxquels ces études font référence. Une mère décrit ainsi les sacrifices qu'elle doit faire pour concilier son travail avec sa vie familiale: Je travaille la nuit pour avoir plus de temps avec mon enfant. L'école, qui sait que je travaille à la maison, me demande d'aller le chercher à tous les midis. J'ai ruiné ma santé avec ça. Malgré tout, je dois continuer à travailler de nuit pour améliorer ma situation financière (44).

Les conditions de vie difficiles des parents entraînent de la fatigue et du stress, affectent leurs pratiques éducatives, nuisent à la discipline et à sa constance. Face à ces conditions, il devient impératif de s'interroger sur leurs effets à long terme. De plus, il faut s'interroger sur les effets négatifs de l'absence de temps des parents, à l'instar des questions que pose leur horaire surchargé, sur la santé des enfants (Kirkey, 2002).

Par ailleurs, sous l'angle de la lutte contre la pauvreté, deux mères mentionnent l'importance qu'a eue pour elles leur retour aux études ou sur le marché du travail: Je suis retournée travailler, ce qui a énormément amélioré ma situation financière. J'avais un stress de moins dans ma vie. Ma qualité de vie s'est également améliorée parce que je peux mieux faire ce que je veux avec mon enfant. J'ai pu améliorer la nourriture et les vêtements (13); 
Je suis retournée à l'école pour nous aider, pour avoir une meilleure job. Je ne lui donne pas autant d'attention qu'avant, parce que je suis à l'école. Sauf quand je suis là, je suis là! (112).

\subsubsection{L'isolement social}

Un parent sur cinq de ce sous-échantillon reconnaît que le fait de ne pas bénéficier de soutien lui fait vivre de l'isolement social. Cet isolement a un effet négatif sur les comportements de leur l'enfant. Le soutien se définit comme étant une interaction entre deux personnes dont l'une éprouve un besoin d'aide et l'autre tente de le combler par des ressources ou par diverses autres formes (Hamel, 2001). Lors de difficultés, le soutien des personnes de l'entourage et des réseaux formels et informels peut avoir un effet tampon (Tarabulsy et Tessier, 1996). À cet égard, trois mères parlent surtout de l'isolement par rapport à leur famille. Le soutien familial paraît indispensable afin de se ressourcer au plan émotionnel, en plus d'apporter une aide concrète: Je me sens seule. J'aimerais avoir l'appui de ma mère. Ma mère m'a beaucoup aidée avec mon fils aîné. Elle n'est plus ici maintenant (09); Je sais qu'il s'ennuie beaucoup de ses grands-parents. Je ne viens pas d'ici. Je n'ai aucune famille dans la région. Il trouve ça très difficile (13).

Puisque la famille est la première source de soutien (Fortin, 1999), il n'est pas surprenant que son absence fasse ressortir un grand sentiment $\mathrm{d}$ 'isolement. Toutefois, quoique ce soutien puisse être fondamental, il peut aussi comporter un effet pervers. À cet effet, Cavell (2000) note que les parents d'enfants ayant des comportements violents vivent moins de contacts positifs et sentent que leur entourage les juge et les critique. Les réseaux sociaux formels et informels des parents deviennent alors une occasion de détresse au lieu d'une source de soutien réel. Les parents cessent d'y recourir par crainte de l'opinion qu'ils pourraient se faire de la situation-problème (Fortin, 1999). Une mère explique ses inquiétudes: Lorsqu'on parle à d'autres, on se fait toujours critiquer et on se fait dire qu'on l'élève mal (44).

\subsubsection{La maladie de la mère}

Une seule mère de ce sous-échantillon considère que sa maladie représente une importante source de stress familial ayant eu un effet négatif sur les comportements de son fils. L'importance de cette situation force à y accorder une attention particulière, en dépit du fait qu'il s'agisse d'une famille intacte: Tout va bien. Il se fait gâter par tout le monde. Il est le plus jeune de toute la gang. Ça fait que tout le monde l'adore (97). 
Lorsque cette mère évoque ses problèmes de santé, elle insiste sur le fait que son enfant souffre beaucoup de cette situation: Ça fait depuis qu'il a 2 ans que je suis bien malade. J'ai eu trois opérations en cinq ans. Ça fait qu'il a toujours vu ça. Il m'a toujours vue malade et faible. Il m'a vue sortir de l'hôpital. Je me souviens, il était fâché contre moi parce que j'étais pas là pour le prendre dans mes bras... Il ne comprenait pas. Il était trop jeune. Je me souviens de ça, là, il était vraiment blessé, pauvre enfant [...]. Je commence juste à reprendre mes forces. Ma dernière opération, c'était au mois d'août dernier. Là, je commence à me remettre sur pied (97). Il est important de spécifier que cette mère a été malade pendant une période d'environ cinq ans, ce qui signifie que son fils a vécu cette situation presque toute sa vie.

En définitive, tout changement ou obstacle important survenant dans la vie des familles semble se répercuter sur celle de l'enfant. Ce dernier est affecté par l'instabilité et toute autre situation entraînant des perturbations familiales (Cavell, 2000; Flanagan, 1999). Les conditions de vie difficiles des familles ont un effet négatif sur les comportements des enfants, une situation sous-estimée dans les écrits. Plus spécifiquement, les longues heures de travail représentent un nouvel aspect de la problématique des comportements violents chez les enfants du préscolaire dont il faut mieux comprendre les effets à long terme. Les stresseurs familiaux ayant un effet négatif sur les comportements des 3 à 6 ans sont présentés au tableau 3.23.

TABLEAU 3.23

Les stresseurs familiaux ayant un effet négatif sur les comportements des 3 à 6 ans $(n=23)$

\begin{tabular}{lcccc}
\hline & \multicolumn{2}{c}{ Garçons } & \multicolumn{2}{c}{ Filles } \\
\cline { 2 - 5 } & $N$ & $d$ & $N$ & $\%$ \\
\cline { 2 - 5 } & 20 & 87,0 & 3 & 13,0 \\
\hline Conflits intrafamiliaux & 14 & 61,0 & 1 & 4,0 \\
\hline Peu de relations avec le père & 13 & 56,0 & & \\
\hline Manque de temps des parents & 11 & 48,0 & 1 & 4,0 \\
\hline Difficulté à concilier travail & 6 & 22,0 & 2 & 8,0 \\
et famille & 5 & 22,0 & & \\
\hline Isolement social & 3 & 13,0 & & \\
\hline Disputes & 3 & 13,0 & & \\
\hline Violence conjugale & 3 & 13,0 & & \\
\hline Absence du père & 1 & 4,0 & 1 & 4,0 \\
\hline Séparation ou divorce & 1 & 4,0 & & \\
\hline Maladie de la mère & & & & \\
\hline
\end{tabular}




\subsection{LES FACTEURS EXPLICATIFS DE LA SITUATION-PROBLÈME À L'ÉCOLE}

Selon les parents de ce sous-échantillon, les facteurs pouvant expliquer la situation-problème de leur enfant montrent que, sans négliger l'importance des stresseurs familiaux, il est crucial d'examiner la situation qui prévaut à l'école, et ce, dès l'entrée à la maternelle et au jardin: La vie, c'est très difficile et ça commence à l'école (108).

\subsubsection{L'attention accordée aux enfants}

Pour expliquer les comportements violents de leur enfant, un consensus semble émerger de la part des parents de ce sous-échantillon à l'effet que $c^{\prime}$ est une manière pour lui d'attirer l'attention: Il requiert toujours mon attention (20); Il a juste besoin d'une attention quelconque [...]. C'est un enfant qui a besoin d'attention (110).

Un peu moins de la moitié des parents croient que si la situation de leur enfant est pire à l'école qu'à la maison, c'est parce que les enseignants ne lui accordent pas toute l'attention requise: Je me suis rendu compte que mon enfant n'avait pas toute l'attention dont il avait besoin. Ça m'a fait un peu de peine. On écrivait des notes dans l'agenda vers la fin de l'année, au mois de mai ou juin, et on avait beau demander des choses à l'avance, il n'y avait aucune réponse. Une fois, je suis arrivée en classe et je me suis rendue compte qu'elle disait aux enfants de mettre des autocollants qui montraient qu'elle avait regardé l'agenda. J'ai trouvé ça absolument ridicule. Les enfants allaient mettre l'agenda sur les genoux de l'enseignante. Elle ne le regardait même pas (01).

\subsubsection{La supervision à l'école}

Dans son ouvrage sur la violence à l'école, Astor (1995) explique qu'elle est surtout présente lorsqu'il y a un manque de supervision de la part des enseignants. Il ajoute que la majorité des comportements violents au préscolaire et au primaire surviennent lorsque la supervision est au minimum, soit lors des récréations.

Le tiers des parents de ce sous-échantillon considèrent que la période des récréations est particulièrement difficile pour leur enfant, car c'est un moment propice à l'émergence et au maintien de ses comportements violents. Pour ces raisons, certains parents préféreraient que l'organisation des récréations soit améliorée: C'est là où tout son fou sort et c'est là en général qu'il se fait punir (99); Ce qui se passe dans la cour d'école... C'était trop grand pour lui. Il se sentait perdu. Je pense qu'il n'y avait pas d'activités dirigées, pas 
assez pour un enfant. Donc, ils sont laissés à eux-mêmes dans la cour [...]. Je trouve que dans les heures de dîner, il n'y a pas assez d'activités. Il y a beaucoup de violence à cause de ça (108).

\subsubsection{La discipline scolaire}

Le tiers des parents de ce sous-échantillon se déclarent en désaccord avec la façon de gérer la classe de l'enseignant et trouvent qu'elle est inadéquate pour leur enfant. Par ailleurs, deux parents estiment que l'enseignant est trop sévère tandis que le même nombre, à l'inverse, jugent qu'il est trop permissif. Enfin, deux autres parents ne critiquent pas la manière d'être de l'enseignant, mais laissent entendre que les différences quant à l'application de la discipline à la maison et à l'école créent de la confusion chez leur enfant.

En général, les deux tiers des parents témoignent qu'ils aimeraient que l'organisation scolaire soit mieux adaptée aux besoins de leur enfant: Ça deorait donner plus à l'enfant au lieu de toujours le structurer. C'est le temps du casse-tête, c'est le temps de ce jeu, c'est le temps de tel truc, tout est minuté pour l'enfant. Je pense que cet enfant-là, il a une certaine révolte en lui. Moi, ce que je pense, c'est que c'est une révolte à tout ce qui est au-dessus de lui, tout ce qu'on décide pour lui en tout temps (99).

\subsubsection{L'étiquetage des enfants}

Le tiers des parents de ce sous-échantillon affirment que le fait que leur enfant est étiqueté comme étant violent n'améliore pas sa situationproblème à l'école: Ils sont rapides pour mettre des étiquettes. C'est un danger. Dès qu'il y en a un qui est hyperactif, ça y est, on ne peut pas travailler avec lui parce qu'il dérange la classe (01); Ça prendrait des enseignants qui n'attendent pas des enfants parfaits (44); Je crois que mon fils, en allant à cette école, il s'est bâti un mauvais nom (80); Sa réputation est faite! (97).

Deux parents ont même retiré leur enfant de l'école ou de la garderie pour l'inscrire dans une autre institution, espérant ainsi solutionner sa situation-problème. Malheureusement, dans bien des cas, le transfert de l'enfant d'une institution à l'autre est loin de faire disparaître ses antécédents scolaires, car son dossier est transféré avec lui, ce qui explique que son étiquetage se poursuit généralement bien au-delà d'une première mauvaise expérience. 


\subsubsection{La stabilité du personnel scolaire}

Près du quart des parents de ce sous-échantillon rapportent diverses situations impliquant des conséquences négatives, suite à un changement de personnel survenu au cours de l'année scolaire. Un d'entre eux affirme que le manque de stabilité du personnel à la garderie scolaire est un phénomène ayant grandement favorisé les comportements violents de son enfant: Pourquoi ça allait pas à la garderie? À chaque fois qu'il y avait une gardienne avec qui ça fonctionnait, elle partait. C'était une nouvelle. Du changement dans cette place-là, c'était terrible. Au niveau des gardiennes, c'était terrible. Aussitôt que mon fils s'adaptait à une nouvelle personne, ça changeait. C'était encore une nouvelle. Alors, ça lui prenait un certain temps à s'adapter. Une fois qu'il était adapté, ça changeait encore (109).

Selon un dernier parent, le changement d'enseignant a aussi influencé négativement les comportements de son fils: Il y avait trop d'élèves dans la classe, bien trop d'élèves. Ils ont alors décidé d'engager une autre enseignante et de diviser la classe en deux. Mon garçon est allé dans l'autre classe. Ça l'a dérangé. Je sens que ça l'a dérangé parce qu'il aimait bien son premier enseignant et qu'il a d̂ิu recommencer à zéro avec l'autre. Je comprends pourquoi ils ont pris cette décision, mais je ne pense pas que c'était judicieux de le faire à ce moment-là (114).

Le changement d'école peut également contribuer à l'émergence de comportements violents. En plus du changement de classe et d'école, il ne faut pas minimiser l'effet négatif lié aux difficultés de communication que l'enfant éprouve en raison qu'il ne maîtrise pas suffisamment la langue d'usage: Le changement d'école anglaise pour une école française a été difficile parce qu'il ne comprend pas bien. Ça déclenche souvent ses colères (108).

En définitive, beaucoup de facteurs sont à considérer lorsque survient une situation-problème à l'école. Ceux-ci se rapportent essentiellement à l'organisation scolaire. Les facteurs explicatifs de la situation-problème à l'école des 3 à 6 ans sont présentés au tableau 3.24.

\section{LES COMPORTEMENTS VIOLENTS DES 6 À 9 ANS}

Dans cette section, nous abordons le passage entre le préscolaire et le primaire. Puis, nous présentons les comportements violents, les problèmes internalisés et externalisés, les problèmes de socialisation, la durée, la fréquence, le lieu et le moment des comportements violents, de même que la trajectoire qu'ils empruntent. En terminant, nous présentons les pratiques éducatives parentales ayant un effet positif et négatif, les 
stresseurs familiaux ayant un effet négatif sur les comportements et les facteurs explicatifs de la situation-problème de l'enfant à l'école, selon les perceptions des parents de ce sous-échantillon.

TABLEAu 3.24

\section{Les facteurs explicatifs de la situation-problème à l'école} des 3 à 6 ans $(n=23)$

\begin{tabular}{lcccc}
\hline & \multicolumn{2}{c}{ Garçons } & \multicolumn{2}{c}{ Filles } \\
\cline { 2 - 5 } & $N$ & $\%$ & $N$ & $\%$ \\
\cline { 2 - 5 } & 20 & 87,0 & 3 & 13,0 \\
\hline Organisation trop rigide & 13 & 57,0 & 2 & 8,0 \\
\hline $\begin{array}{l}\text { Manque d'attention } \\
\text { de l'enseignant }\end{array}$ & 9 & 39,0 & 1 & 4,0 \\
\hline $\begin{array}{l}\text { Manque de supervision } \\
\text { (récréation) }\end{array}$ & 8 & 35,0 & & \\
\hline Gestion de classe inadéquate & 7 & 31,0 & 1 & 4,0 \\
\hline Étiquetage des enfants & 6 & 26,0 & 2 & 8,0 \\
\hline Changement de personnel & 5 & 22,0 & & \\
\hline Discipline trop sévère & 2 & 8,0 & & \\
\hline Discipline trop permissive & 2 & 8,0 & & \\
\hline Changement de classe & 1 & 4,0 & & \\
ou d'école & &
\end{tabular}

\subsection{LE PASSAGE dU PRÉSCOLAIRE AU PRIMAIRE}

À première vue, le monde de l'enfant d'âge scolaire peut sembler assez similaire à celui de l'enfant du préscolaire. La famille, les amis et l'école font déjà partie de son univers social. Toutefois, la nature des relations que l'enfant entretient avec les différents éléments de son environnement est le siège de changements importants lors de son passage du préscolaire au primaire. L'une des différences majeures réside dans l'importance respective des parents et des pairs comme agents de socialisation. De plus, à l'âge scolaire, les interactions sociales avec les pairs augmentent en fréquence, alors que celles avec les parents occupent de moins en moins de place dans la vie de l'enfant (Rubin, 1996). 


\subsection{LES COMPORTEMENTS VIOLENTS}

Tout comme dans le cas des enfants de 3 à 6 ans, nous avons divisé les comportements violents chez les enfants de 6 à 9 ans en deux catégories selon la Conflict Tactics Scale de Straus et Gelles (1990). La première regroupe la violence physique et la seconde, la violence verbale. Cette dernière regroupe deux sous-catégories, soit la violence verbale sans geste et avec gestes. La violence verbale sans geste comprend également la violence symbolique.

\subsubsection{La violence physique}

La violence physique est beaucoup plus fréquente que la violence verbale chez les 37 enfants de ce sous-échantillon. Les deux tiers des garçons frappent et tapent les autres: À la garderie, il y avait un bâton de baseball en plastique et il a frappé un élève en arrière des jambes avec (90); Il va me frapper (94.1); Des fois, quand je vais le taper sur la main, il va me retaper, mais c'est pas... Tu sais, il a peur. C'est juste pour dire qu'il a le dernier mot (96).

Un peu moins des deux tiers de ces enfants se battent et donnent des coups de poing. Plus de la moitié donnent des coups de pied, tandis que l'autre moitié mordent: Il frappe, casse des choses et donne des coups de pied à sa sceur (37); Il va me mordre (94.1); Elle a «kické» son frère (95). Également, le tiers des enfants de ce sous-échantillon malmènent ou intimident leurs pairs. Finalement, quelques-uns poussent. Les comportements de violence physique des 6 à 9 ans sont présentés au tableau 3.25.

TABLEAU 3.25

Les comportements de violence physique des 6 à 9 ans $(n=37)$

\begin{tabular}{lcccc}
\hline & \multicolumn{2}{c}{ Garçons } & \multicolumn{2}{c}{ Filles } \\
\cline { 2 - 5 } & $\boldsymbol{N}$ & $\%$ & $\boldsymbol{N}$ & $\%$ \\
\cline { 2 - 5 } & 34 & 92,0 & 3 & 8,0 \\
\hline Frappent et tapent & 24 & 65,0 & & \\
\hline Se battent & 22 & 59,5 & & \\
\hline Donnent des coups de poing & 21 & 57,0 & 1 & 2,5 \\
\hline Donnent des coups de pied & 19 & 51,0 & 1 & 2,5 \\
\hline Mordent & 18 & 48,5 & & \\
\hline Malmènent ou intimident & 13 & 35,0 & & \\
\hline Poussent & 2 & 5,5 & 1 & 2,5 \\
\hline
\end{tabular}




\subsubsection{La violence verbale avec ou sans gestes}

Pour ce qui est de la violence verbale sans geste, les trois quarts des enfants à l'étude blâment le plus souvent les autres: C'est toujours la faute des autres (05); Il a blâmé un autre enfant alors que c'était lui (23); C'est pas de sa faute, c'est jamais lui qui commence (83).

Les deux tiers des enfants insultent et un peu plus du tiers d'entre eux font des menaces: Dis-le pas à Madame X sinon, tu vas voir ce que je vais te faire (90); Il avait dit: "Je m'en vais pis je reviens pas!» Il a commencé à mettre son linge dans un sac (94.1).

Également, le quart des enfants utilisent un langage vulgaire pouvant porter atteinte à l'intégrité des personnes visées: La façon de parler. Il est aussi bête à la maison que dehors avec ses amis. Il n'a aucun respect (83); Il y a des mots vraiment, là, comme "bitch", "son of a bitch», des choses comme ça (90).

Quant à la violence verbale avec gestes, un peu plus de la moitié des enfants détruisent leurs jouets, ceux des autres, ou même d'autres objets qui leur tombent sous la main: Son travail, il va le déchirer (38); Il détruit ses jouets (88); Ce qu'il a devant lui, s'il est pas content, il va le déchirer (90).

Un peu moins du quart frappent des objets, les lancent comme des projectiles, ou même leur donnent des coups de pied: Il a lancé une chaise sur la porte du garde-robe (05); Il a "garoché» quelque chose par la fenêtre (90); Il va donner des coups de pied sur le mur (96). Les comportements de violence verbale avec ou sans gestes des 6 à 9 ans sont présentés au tableau 3.26.

TABleau 3.26

Les comportements de violence verbale avec ou sans gestes des 6 à 9 ans $(n=37)$

\begin{tabular}{lcccc}
\hline & \multicolumn{2}{c}{ Garçons } & \multicolumn{2}{c}{ Filles } \\
\cline { 2 - 5 } & $\boldsymbol{N}$ & $\%$ & $\boldsymbol{N}$ & $\%$ \\
\cline { 2 - 5 } & 34 & 92,0 & 3 & 8,0 \\
\hline Blâment & 25 & 67,5 & 3 & 8,0 \\
\hline Insultent & 22 & 59,5 & 2 & 5,5 \\
\hline Détruisent des objets & 20 & 54,0 & 1 & 2,5 \\
\hline Font des menaces & 14 & 38,0 & 1 & 2,5 \\
\hline Utilisent un langage vulgaire & 8 & 21,5 & 1 & 2,5 \\
\hline Frappent des objets & 5 & 13,5 & 1 & 2,5 \\
\hline Lancent des objets & 5 & 13,5 & 1 & 2,5 \\
\hline Donnent des coups de pied & 5 & 13,5 & 1 & 2,5 \\
aux objets & & & &
\end{tabular}




\subsubsection{Les problèmes internalisés}

Quant aux problèmes internalisés, l'anxiété et l'insécurité ressortent chez plus des trois quarts des enfants de ce sous-échantillon: Il a le gros défaut de se fouiller dans le nez, mais ça c'est un geste nerveux, comme de se ronger les ongles, parce que dès qu'il s'en rend compte, il arrête tout de suite. Ça le rend bien malheureux, cette manie-là. Lui, il dit que c'est à cause de ça qu'il n'a pas d'amis (11.1); Il devient très nerveux quand il est à l'école (15); Il ne va pas loin sans moi. Il doit savoir où je suis en tout temps (58); Mon enfant est très «insécure» (85); Il a peur que je parte. Il est bien «insécure» (86); Quand il est vraiment stressé, il se met les deux mains sur la tête. Là, il faut que je lui dise de relaxer (90); Il fait toujours des cauchemars. C'est rien que de la violence dans son cauchemar. C'est toujours comme quelque chose avec moi et mon partenaire. Qu'il m'a tuée durant la nuit! (94.1).

Les sentiments dépressifs et la tristesse apparaissent également chez la moitié des garçons: Il est souvent triste (06); Je pensais qu'il était déprimé, ou même qu'il était morbide, parce qu'il fait tout le temps des dessins de squelettes, de têtes de mort et de vampires (12.1); Il se met facilement à pleurer (22); Il devient en peine (86); Il va pleurer. Il va regretter ses gestes. Il va pleurer comme un bébé parfois (90); À l'école, on m'a dit qu'il est toujours triste, qu'il a quelque chose dans son cœur qu'il veut pas dire. Il veut pas parler (105).

Le tiers des enfants manquent d'estime de soi et de confiance en soi. Également, quelques-uns d'entre eux s'isolent, se blâment ou entretiennent des idées suicidaires: Là il va dire: "Qu'est-ce que j'ai fait mon Dieu? ». Il dit qu'il ne comprend pas pourquoi il agit comme ça (90); Il a déjà allumé le plafond en feu... C'était durant la nuit. Il a pris un briquet et il a allumé un rouleau de papier de toilette. Il voulait qu'on meure tous ensemble! (12.1).

Une dernière petite fille va exprimer sa souffrance en posant des gestes de violence physique contre soi: Elle se frappe. Elle va se frapper sur la tête et sur le ventre (107). Les problèmes internalisés des 6 à 9 ans sont présentés au tableau 3.27.

\subsubsection{Les problèmes externalisés}

La presque totalité des enfants de ce sous-échantillon s'opposent à leurs parents. De plus, la majorité d'entre eux n'écoutent pas les consignes: Il n'écoute jamais rien de ce que je dis (84); Il n'écoute pas du tout (94.1).

Les sautes d'humeur, le chialage et l'impulsivité sont présents chez les trois quarts d'entre eux: Il est capable de changer de décision vite (06); Des fois, il ne sait plus ce qu'il fait. Sans être violent, c'est la façon dont il réagit. 
D'une minute à l'autre, il change complètement (43); Ah oui! Il «chiale» [se plaint] tout le temps. "Ah! Ça me tente pas d'aller chez grand-maman! Pourquoi est-ce qu'on s'en va là? » (soupir) (96).

TABLEAU 3.27

Les problèmes internalisés des 6 à 9 ans $(n=37)$

\begin{tabular}{lcccc}
\hline & \multicolumn{2}{c}{ Garçons } & \multicolumn{2}{c}{ Filles } \\
\cline { 2 - 5 } & $\boldsymbol{N}$ & $\%$ & $\boldsymbol{N}$ & $\%$ \\
\cline { 2 - 5 } & 34 & 92,0 & 3 & 8,0 \\
\hline Éprouvent de l'anxiété & 30 & 82,0 & 1 & 2,5 \\
\hline Se sentent insécures & 30 & 82,0 & 1 & 2,5 \\
\hline Ont des sentiments dépressifs & 18 & 48,5 & & \\
\hline Manquent d'estime de soi & 10 & 27,0 & 3 & 8,0 \\
\hline Manquent de confiance en soi & 8 & 21,5 & 2 & 5,5 \\
\hline S'isolent & 3 & 8,0 & & \\
\hline Se blâment & 2 & 5,5 & & \\
\hline Entretiennent des idées suicidaires & 2 & 5,5 & & \\
\hline Pose des gestes contre soi & \multicolumn{5}{c}{1} & 2,5 \\
\hline
\end{tabular}

En plus de ces comportements oppositionnels, la suractivité est présente dans les mêmes proportions: Il est actif et il a des fois des problèmes internes, c'est difficile pour lui de s'asseoir tranquillement (38); S'asseoir et écouter, c'est difficile pour mon enfant (84); Il ne peut pas s'asseoir, il court toujours (94.1).

Également, les deux tiers des parents affirment que leur enfant est influençable et que ses agissements découlent de ceux des pairs: Tous les gars se battent et lui, il fait pareil (11.1); La semaine passée, il y a un petit ami qui a dit à $X$ : "Donne un coup de poing à ton frère!». Mon enfant l'a frappé dans le ventre (12.1); Son comportement est influencé négativement par d'autres enfants (22); Il est le type qui suit les autres (32); Il dit toujours que c'est l'autre qui a commencé, puis que c'est lui qui a dit de le faire (94.1); Elle voit agir les plus vieux. Puis, elle voit aussi son frère (106).

Par ailleurs, la frustration est palpable chez la moitié des enfants: Elle se frustre facilement (95).

Les parents notent également le refus de se soumettre à l'autorité: Il refuse d'écouter et de se soumettre à l'autorité (15); Son comportement envers l'autorité $n^{\prime} e s t$ pas correct (81). Le tiers des parents déclarent que leur enfant fait des crises: Il fait sa crise (83); Il fait des crises de nerfs (88). 
Dans cinq cas, les parents déclarent que, lors de crises, il est difficile pour leur enfant, voire impossible, de se contrôler: Il a de la difficulté à se contrôler (46); Il était hors de contrôle (84); Il n'est plus capable de se retenir (96).

D'autres comportements externalisés sont également présents, tels que faire des colères, mentir et voler: Il va mentir pour ses devoirs. Il va mentir: "Ben, j'ai pas touché à ça!». Des choses qu'il a pas besoin de mentir, il va mentir quand même (05); Il a des excès de rage et de colère (47); Il n'est pas capable de dire la vérité (83); Il va voler (94.1).

L'impatience est aussi présente: Il a de la difficulté à accepter qu'il doive attendre pour avoir quelque chose (62).

Également, deux garçons ont fait des fugues et un dernier triche au jeu. Les problèmes externalisés des 6 à 9 ans sont présentés au tableau 3.28.

TABLeau 3.28

Les problèmes externalisés des 6 à 9 ans $(n=37)$

\begin{tabular}{lcccc}
\hline & \multicolumn{2}{c}{ Garçons } & \multicolumn{2}{c}{ Filles } \\
\cline { 2 - 5 } & $N$ & $\%$ & $N$ & $\%$ \\
\cline { 2 - 5 } & 34 & 92,0 & 3 & 8,0 \\
\hline S'opposent à l'adulte & 33 & 89,5 & 3 & 8,0 \\
\hline N'écoutent pas les consignes & 28 & 75,5 & 3 & 8,0 \\
\hline Ont des sautes d'humeur & 26 & 70,0 & 1 & 2,5 \\
\hline «Chialent» (se plaignent) & 26 & 70,0 & 3 & 8,0 \\
\hline Font preuve d'impulsivité & 25 & 67,5 & 1 & 2,5 \\
\hline Font preuve de suractivité & 25 & 67,5 & 1 & 2,5 \\
\hline Se laissent influencer & 21 & 57,0 & 1 & 2,5 \\
\hline Éprouvent de la frustration & 17 & 46,0 & 1 & 2,5 \\
\hline Refusent de se soumettre à l'autorité & 17 & 46,0 & 1 & 2,5 \\
\hline Font des crises & 11 & 30,0 & & \\
\hline Font des colères & 6 & 16,0 & & \\
\hline Manquent de contrôle de soi & 5 & 13,5 & & \\
\hline Mentent & 5 & 13,5 & & \\
\hline Volent & 5 & 13,5 & & \\
\hline Font preuve d'impatience & 3 & 8,0 & 1 & 2,5 \\
\hline Fuguent & 2 & 5,5 & & \\
\hline Triche & 1 & 2,5 & & \\
\hline
\end{tabular}




\subsubsection{Les problèmes de socialisation}

Le quart des enfants de ce sous-échantillon qui éprouvent des problèmes de socialisation n'ont pas d'amis, c'est pourquoi beaucoup jouent plus souvent seuls qu'en groupe, malgré que les parents affirment que leur enfant aime beaucoup jouer avec les autres ou même le préfère. Afin de parvenir à se faire des amis, les enfants utilisent diverses stratégies: Il a toujours quelque chose avec lui, comme un «skateboard», un «bicycle» ou une trottinette. Pour se faire des amis, il va leur dire: "Viens jouer! Regarde mon "skate", veux-tu l'essayer?" (96).

D'autres enfants sont portés à s'isoler et leurs parents ne parviennent pas facilement à les sortir de cet isolement: Il n'est pas capable de m'en parler (84); Il ne parle plus comme avant pour m'expliquer comment il se sent (85); Il est fermé et je ne réussis pas toujours à le sortir de lui-même (90); Aller s'asseoir dans son petit coin (95).

Deux autres parents affirment que leur enfant se sent rejeté: Il se sent à part des autres, ça le rend triste (11.1); Parfois, quand il y a des travaux de groupe, $X$ n'est pas souvent choisi par ses amis. Il l'a remarqué et il nous en a parlé (12.1).

Également, quelques enfants se font pousser, frapper ou agacer par les autres. Les problèmes de socialisation des 6 à 9 ans sont présentés au tableau 3.29.

TABLEAU 3.29

Les problèmes de socialisation des 6 à 9 ans $(n=37)$

\begin{tabular}{lcccc}
\hline & \multicolumn{2}{c}{ Garçons } & \multicolumn{3}{c}{ Filles } \\
\cline { 2 - 5 } & $N$ & $\%$ & $N$ & $\%$ \\
\cline { 2 - 5 } & 34 & 92,0 & 3 & 8,0 \\
\hline Jouent seuls & 9 & 24,0 & 1 & 2,5 \\
\hline Ne fonctionnent pas en groupe & 5 & 13,5 & 1 & 2,5 \\
\hline S'isolent & 5 & 13,5 & & \\
\hline Se sentent rejetés & 2 & 5,5 & & \\
\hline Se font pousser par les autres & 2 & 5,5 & & \\
\hline Se font frapper ou taper & 2 & 5,5 & & \\
\hline Se fait agacer & 1 & 2,5 & & \\
\hline
\end{tabular}




\subsection{LA DURÉE ET LA FRÉQUENCE DES COMPORTEMENTS VIOLENTS}

Parmi les indices qui montrent que les comportements violents durent depuis un certain temps, les parents sont en mesure de comparer différentes périodes au cours desquelles ces comportements augmentent ou diminuent: Mon enfant a encore des épisodes où il a des mauvais comportements. Ce n'est plus régulier comme c'était il y a trois mois (37); Cette année, il est pas pire. Les autres années, oui, ça arrivait pas mal souvent. Au moins une fois par mois. Cette année, il a eu une mauvaise période. Puis, ça a arrêté (96).

Par ailleurs, un parent constate que les comportements violents de son enfant sont davantage présents au début de l'année scolaire: Quand l'école commence, elle a plus de "misère» [difficulté] [...]. Elle a des petites habitudes. Puis des fois, elle peut être un peu plus agressive (95).

Pour un autre, ceux-ci sont apparus au cours de la première année de scolarisation: C'est à partir de la première année que tout a commencé (52).

Un dernier parent affirme qu'ils ont débuté vers l'âge de 5 ans: En maternelle et au début du jardin, il n'y avait pas de problèmes avec mon enfant. Vers la fin, il y a eu un changement de direction et il se faisait suspendre pour n'importe quoi (84).

Quant à la fréquence des comportements violents, elle varie considérablement d'un enfant à l'autre, allant d'une fois par jour à quelques fois par année. Ainsi, six parents déclarent que leur enfant a des comportements violents une fois par jour, huit d'entre eux parlent de plusieurs fois par semaine. Pour six autres, il en est question une fois par semaine, tandis que six autres les constatent plusieurs fois par mois. Chez cinq enfants, de tels comportements se produisent une fois par mois; chez cinq autres, de manière épisodique. La fréquence des comportements violents des 6 à 9 ans est présentée au tableau 3.30.

\subsection{LE LIEU ET LE MOMENT DES COMPORTEMENTS VIOLENTS}

En ce qui a trait au lieu où les comportements de violence s'exercent, plus des trois quarts des parents affirment que c'est davantage à l'école qu'à la maison: À la maison, il est plus ouvert aux changements. À la maison, les changements sont "l'fun» [agréables]. À l'école, un changement c'est par rapport aux travaux et il prend ça comme de la pression. Il n'aime pas vraiment être à l'école. Tout est plus facile à la maison (38). 
TABLEAu 3.30

\section{La fréquence des comportements violents des 6 à 9 ans $(n=37)$}

\begin{tabular}{lcccc}
\hline & \multicolumn{2}{c}{ Garçons } & \multicolumn{2}{c}{ Filles } \\
\cline { 2 - 5 } & $\boldsymbol{N}$ & $\%$ & $\boldsymbol{N}$ & $\%$ \\
\cline { 2 - 5 } & 34 & 92,0 & 3 & 8,0 \\
\hline Une fois par jour & 6 & 16,0 & & \\
\hline Plusieurs fois par semaine & 8 & 21,5 & & \\
\hline Une fois par semaine & 6 & 16,0 & & \\
\hline Plusieurs fois par mois & 4 & 11,0 & 2 & 5,5 \\
\hline Une fois par mois & 5 & 13,5 & & \\
\hline De manière épisodique & 5 & 13,5 & 1 & 2,5 \\
\hline
\end{tabular}

Les parents constatent que les comportements violents des enfants sont pires à l'école puisqu'ils sont en contact avec les autres élèves: Ce n'est pas pareil à la maison parce qu'elle n'a pas d'amies pour la déranger ici (12); Il est moins physique à la maison. À l'école, c'est plus souvent à cause des autres enfants. À la maison, il frappe moins (43); ̀̀ la maison, il est avec des adultes. Son comportement est différent lorsqu'il se trouve avec d'autres enfants (58); Je pense que c'est plutôt le contact avec les autres enfants qui le dérange (90).

À l'inverse, le quart des parents constatent que leur enfant fait davantage preuve de comportements violents à la maison qu'à l'école. Cette situation est observable lorsque le degré de tolérance est moins élevé dans le milieu scolaire que dans le milieu familial: À l'école, mon enfant va mieux. À la maison, il surveille moins son comportement, car il est plus à l'aise ici (10); C'est moins intense à la maison puisqu'il est plus libre. Il n'est pas dans une classe. C'est aussi qu'on dit non à l'école (88); Il me semble qu'il se retient un peu plus à l'école (96); Je pense qu'elle est différente à la maison. Elle est moins gênée. Elle est un peu plus gênée à l'école (106).

Lorsque les enfants ont moins de comportements violents à la maison qu'à l'école, les parents y voient plusieurs raisons: C'est différent à la maison, parce que maman est ici. Puis, à l'école il va s'essayer plus (06); Non, parce que les parents sont là. Donc, il n'a pas ces comportements (49); En notre présence, il n'y a pas d'agression physique (62); C'est moins pire à la maison parce que je le tiens un peu plus serré. Quand il n'est pas à l'école, il est calme (86); À l'école, elle peut se faire "chicaner» [réprimander] par les profs, mais c'est pas sévère comme à la maison (95).

À la maison, l'enfant reçoit ainsi davantage d'attention de la part de ses parents: Il va être plus tranquille vu qu'il joue seul (05); C'est moins pire à la maison. Quand c'est un à un, ça va (23); À la maison, je le laisse être comme il est (48); Il est plus attentif. C'est un sur un (85). 
Par ailleurs, lorsque les comportements violents sont plus fréquents à la maison qu'à l'école, les parents reconnaissent certains moments de la journée où ils sont davantage présents: C'est surtout durant les devoirs (06); À la maison, je pense que c'est peut-être le matin, de temps en temps. Mais, c'est plus le soir qu'il va m'exaspérer (52). Maintenant, moi on dirait que je mange tous les coups: le soir et le matin (90).

À l'inverse, lorsqu'ils sont plus fréquents à l'école qu'à la maison, chez le tiers des enfants, les comportements violents surviennent à l'heure du dîner et dans la cour d'école: C'est plutôt dans la cour d'école que dans la classe (05); Les problèmes, c'était dans la cour d'école (06); Ce que je vois ou que je ressens le plus à l'école, c'est dans le temps de l'heure du dîner. C'est de la grosse merde là-dedans (12.1); Il crache sur le conducteur d'autobus, frappe la surveillante lors du dîner (22); Possiblement, parce que c'est dans ce temps-là qu'elle a eu ses deux feuilles bleues. Toujours à la récréation (106). Le lieu et le moment des comportements violents des 6 à 9 ans sont présentés au tableau 3.31.

TABLeAu 3.31

\section{Le lieu et le moment des comportements violents} des 6 à 9 ans $(n=37)$

\begin{tabular}{lcccc}
\hline & \multicolumn{2}{c}{ Garçons } & \multicolumn{2}{c}{ Filles } \\
\cline { 2 - 5 } & $\boldsymbol{N}$ & $\%$ & $\boldsymbol{N}$ & $\%$ \\
\cline { 2 - 5 } & 34 & 92,0 & 3 & 8,0 \\
\hline$\grave{\text { À l'école }}$ & 23 & 62,0 & 3 & 8,0 \\
\hline Lors des récréations & 7 & 19,0 & 2 & 5,5 \\
\hline Lors du dîner & 4 & 11,0 & 1 & 2,5 \\
\hline À la fin de l'après-midi & 1 & 2,5 & & \\
\hline À la maison & 11 & 30,0 & & \\
\hline Le matin & 3 & 8,0 & & \\
\hline Lors des devoirs & 2 & 5,5 & & \\
\hline Le soir & 2 & 5,5 & & \\
\hline
\end{tabular}

\subsection{LA TRAJECTOIRE DES COMPORTEMENTS VIOLENTS}

Les deux tiers des parents déclarent que les comportements violents de leur enfant ont coïncidé avec le début de la première année scolaire: C'est depuis son entrée à l'école, en première année (11); Quand il est allé à la maternelle, ce n'était pas trop mal, car c'était surtout du jeu. En première année, ç'a été le 
traumatisme (12.1); Mais c'est surtout au cours de sa première année que ça a été le plus mal. Il n'a pas été à la garderie (48); À la transition entre les deux écoles. Depuis le début de la $1^{r e}$ année (50); C'est quand l'école a commencé. Avant ça, non, non. Mon Dieu! X était la meilleure petite fille que tu pouvais avoir. «Believe me or not", j'ai eu plus de troubles lorsqu'elle avait cinq ans que quand elle avait deux ou trois ans (95); Depuis qu'elle a commencé l'école. Je pense qu'elle a fait un $180^{\circ}$. Quand elle a commencé, elle était une petite fille très fine. Puis, elle a «viré à l'envers » [complètement changé] (107).

Pour d'autres parents, $c^{\prime}$ est en deuxième ou en troisième année que les comportements violents sont apparus. Finalement, trois parents admettent que les comportements violents de leur enfant étaient présents bien avant l'entrée en première année: J'ai seulement pensé qu'il était actif. Il grimpait partout et écrivait sur les murs. À la maison, c'était moins pire parce qu'il n'était pas en interaction avec les autres (23); Il a un caractère un peu difficile. Il faut toujours faire attention avec lui (11); Depuis qu'il est en maternelle, sur le bulletin, le développement personnel et social, c'est pas fort (90).

Par ailleurs, trois parents affirment que les comportements violents de leur enfant n'ont pas cessé de s'amplifier depuis la première année, alors qu'un peu moins du tiers d'entre eux déclarent qu'ils n'ont pas cessé de s'améliorer jusqu'à aujourd'hui: Ça c'est l'année passée. Mais là, ça fait un mois et demi qu'il ne s'est rien passé... Je touche du bois. Tout va bien. Mais, cette année, ça a été super! (06); Son comportement s'est beaucoup amélioré depuis le début de l'année (37); Maintenant, c'est excellent. Depuis les deux derniers mois, il ne fait plus rien de mal (50). La trajectoire des comportements violents des 6 à 9 ans est présentée au tableau 3.32 .

TABLEAU 3.32

La trajectoire des comportements violents des 6 à 9 ans $(n=37)$

\begin{tabular}{lrrrr}
\hline & \multicolumn{2}{c}{ Garçons } & \multicolumn{2}{c}{ Filles } \\
\cline { 2 - 5 } & $\boldsymbol{N}$ & $\%$ & $\boldsymbol{N}$ & $\%$ \\
\cline { 2 - 5 } & 34 & 92,0 & 3 & 8,0 \\
\hline Avec l'entrée en première année & 21 & 57,0 & 2 & 5,5 \\
\hline Avec l'entrée en deuxième année & 5 & 13,5 & 1 & 2,5 \\
\hline Avec l'entrée en troisième année & 5 & 13,5 & & \\
\hline Avant l'entrée au primaire & 3 & 8,0 & & \\
\hline Pires depuis la première année & 3 & 8,0 & & \\
\hline Mieux aujourd'hui & 10 & 30,0 & 1 & 2,5 \\
\hline
\end{tabular}




\subsection{LeS PRATIQUES ÉdUCATIVES PARENTALES}

Tout comme les parents des 3 à 6 ans, ceux des 6 à 9 ans ont recours à des pratiques éducatives ayant un effet parfois positif, parfois négatif, afin de contrer les comportements violents de leur enfant. Il apparaît paradoxal dans ce contexte que les parents ressentent un fort sentiment de compétence éducative. Ce constat va dans le sens des observations de Trudelle et Montambault (1994) à savoir que la désirabilité sociale fait en sorte que beaucoup de parents se déclarent satisfaits de la manière dont ils $s^{\prime} y$ prennent avec leur enfant, en omettant de nommer certaines de leurs pratiques éducatives ayant un effet négatif chez leur enfant.

\subsubsection{Les pratiques ayant un effet positif}

Parmi les pratiques éducatives parentales ayant un effet positif, les trois quarts des parents de ce sous-échantillon dialoguent avec leur enfant et leur expliquent ce qui est acceptable comme comportement. Dans les mêmes proportions, $\mathrm{d}^{\prime}$ autres mentionnent qu'ils utilisent toujours la même technique, soit le retrait et la perte de privilèges. Également, le tiers d'entre eux utilisent le renforcement positif, soit des compliments, des sorties ou des cadeaux: À un moment donné, une travailleuse sociale m'avait donné des techniques de récompense (86).

Quelques autres parents donnent des tâches supplémentaires à leur enfant ou font appel à la technique 1-2-3. Finalement, la mère d'une dernière fille déclare maintenir toujours la même routine. Les pratiques éducatives parentales ayant un effet positif chez les 6 à 9 ans sont présentées au tableau 3.33.

\subsubsection{Les pratiques ayant un effet négatif}

Les parents nomment des pratiques éducatives qui leur semblent avoir un effet négatif chez leur enfant. La moitié d'entre eux affirment qu'ils n'interviennent pas toujours lorsqu'ils sont fatigués, en dépit que seule la constance représente une pratique éducative considérée positive, selon Angelino et Meyer (2001): À un moment donné, quand je suis à bout, je ferme les yeux (88); Des fois, $j^{\prime}$ 'suis assez «tannée» [j'en ai assez] de discuter, que là je vais dire oui, parce que je suis trop «tannée» [j'en ai assez] de l'entendre (106).

À l'inverse, d'autres parents avouent que lorsqu'ils sont fatigués, ils interviennent trop ou trop vite, et ce, surtout lorsqu'ils sont à bout: Non. C'est le contraire, quand je suis fatigué, je réagis trop vite (62); Quand c'est de la fatigue, je vais crier deux fois plus fort (84). 
TABLEAU 3.33

Les pratiques éducatives parentales ayant un effet positif chez les 6 à 9 ans $(n=37)$

\begin{tabular}{lcccc}
\hline & \multicolumn{2}{c}{ Garçons } & \multicolumn{2}{c}{ Filles } \\
\cline { 2 - 5 } & $\boldsymbol{N}$ & $\boldsymbol{\%}$ & $\boldsymbol{N}$ & $\boldsymbol{\%}$ \\
\cline { 2 - 5 } & 34 & 92,0 & 3 & 8,0 \\
\hline Dialogue & 25 & 67,5 & 2 & 5,5 \\
\hline Toujours la même technique & 24 & 65,0 & 1 & 2,5 \\
\hline Retrait & 22 & 59,5 & 2 & 5,5 \\
\hline Perte de privilèges & 19 & 51,0 & 2 & 5,5 \\
\hline Renforcement positif & 12 & 32,5 & 2 & 5,5 \\
\hline Tâches supplémentaires & 3 & 8,0 & 1 & 2,5 \\
\hline Technique 1-2-3 & 3 & 8,0 & & \\
\hline Maintien d'une routine & \multicolumn{5}{c}{1} & 2,5 \\
\hline
\end{tabular}

Crier paraît être une pratique coercitive ayant un effet négatif chez l'enfant. Le tiers des parents qui y ont recours essaient de ne plus le faire: Si je crie ou quelque chose comme ça, il va juste empirer (05); Crier, pour lui, c'est très violent, ça ne fonctionne pas. C'est comme le frapper (38); Lorsque je lève la voix ou que je crie, il m'ignore (48). À l'inverse, un parent considère que le fait de parler calmement à son enfant ne donne pas de bons résultats: Tant que je ne crie pas, il ne bouge pas (86).

Également, le quart des parents déclarent ne pas avoir de discipline assez stricte: Dans certaines choses, on n'intervient pas. On n'intervient pas partout. C'est sûr qu'il y a toujours un peu de laxisme de notre part (02); Quand il se «chicane» [chamaille] avec sa sœur, je n'interviens pas trop, car ça bâtit leur caractère (22); Je me suis fait dire de ne pas le punir lorsqu'il revient de l'école, parce qu'il a déjà eu une conséquence. Il ne faut pas le punir deux fois pour la même affaire (81).

Ce style de parentage, habituellement reconnu comme étant libéral / permissif, crée chez l'enfant des problèmes de discipline (Cowie, 2000; McCain et Mustard, 1999). Un second style parental souvent associé aux enfants ayant des comportements violents est dit autoritaire (Day, Golench, MacDougall et Beals-Gonzales, 1995; Ladd, 1999; Rubin, 1996).

Parmi les autres pratiques coercitives que les parents utilisent, ceux-ci admettent parfois frapper, taper, pousser leur enfant ou le retenir par le bras: Parfois, je suis un peu violent avec lui. Je le brasse (02); Si X en a assez de $Y$, il lui donnera une bonne volée et il le laissera tranquille. Dans le fond, c'est de ça dont il a besoin (12.1); L'événement déclencheur a été une tape sur l'oreille 
qu'il a reçue de son père. Il s'est mis à saigner parce qu'il avait une infection (22); Le retenir par le bras lorsqu'il se fâche. Ça n'a pas fonctionné (85); Après la troisième fois que j'avais entendu ça, je l'ai poussé au point qu'elle est tombée par terre (95).

Les punitions abusives font également l'objet de tentatives de la part des parents dont deux avouent qu'elles peuvent s'avérer trop longues: $S i$ la punition dure une semaine... (83). D'autres mettent leur enfant à genoux ou dans un coin: Des fois, il doit se mettre à genoux pour réfléchir (10); Va dans le coin. Pas juste dans la chambre. Debout. Face au mur. Pas le dos au mur, la face au mur! (95).

Pour un autre parent, il s'agit d'avoir mis à quelques reprises du savon dans la bouche de son enfant: S'il dit des méchants mots, ça m'est arrivé d'y mettre du savon dans la bouche (90).

Un parent affirme avoir fait de la discipline qui a pu s'avérer traumatisante psychologiquement pour son enfant: J'ai fait beaucoup de discipline, sans violence, mais peut-être traumatisante psychologiquement (12.1).

Nous classons ce genre de mesures disciplinaires dans la violence verbale avec gestes: J'ai totalement démoli la chambre et le mobilier. Je n'arrivais plus à me contrôler. Je ne les ai pas tapés parce que je suis contre la violence. Ils se sont mis à deux contre $Y$ et j'avais l'impression que je devenais folle. À un moment, $X$ a dit à $Y:$ "Toi, je vais te tuer». J'ai décidé d'enlever le matelas de $X$. Il a couché par terre toute la fin de semaine (12.1). Les pratiques éducatives parentales ayant un effet négatif chez les 6 à 9 ans sont présentées au tableau 3.34.

\subsection{LES STRESSEURS FAMILIAUX}

Tout comme dans le cas des 3 à 6 ans, les comportements violents des 6 à 9 ans ont un effet négatif sur la vie familiale et vice versa. Selon les parents de ce sous-échantillon, les conflits intrafamiliaux, le peu ou l'absence de relations avec le père et le manque de temps des parents sont les stresseurs familiaux qui semblent avoir le plus d'effets négatifs sur les comportements de leur enfant.

\subsubsection{Les conflits intrafamiliaux}

Chez les 6 à 9 ans, les conflits intrafamiliaux englobent les disputes, la séparation ou le divorce et la violence conjugale. À ce sujet, un peu plus $\mathrm{du}$ tiers des parents constatent que de fortes disputes dans le couple ont eu des répercussions importantes sur l'enfant. De telles disputes ont mené à la rupture de couples, ce qui a amené l'enfant à se comporter de manière 
encore plus violente: Mon mari et moi, on parlait assez fort. Lorsque je disais que c'était pas le temps, parce que le jeune était alentour, on continuait quand même nos discussions. C'était malheureusement toujours avec X autour (81).

TABLEAu 3.34

Les pratiques éducatives parentales ayant un effet négatif chez les 6 à 9 ans $(n=37)$

\begin{tabular}{llccc}
\hline & \multicolumn{2}{c}{ Garçons } & \multicolumn{2}{c}{ Filles } \\
\cline { 2 - 5 } & $N$ & $\%$ & & $\%$ \\
\cline { 2 - 5 } & 34 & 92,0 & 3 & 8,0 \\
\hline N'interviennent pas toujours & 18 & 48,5 & 1 & 2,5 \\
\hline Crient & 12 & 32,5 & 1 & 2,5 \\
\hline N'ont pas de discipline & 9 & 24,0 & & \\
assez stricte & 5 & 13,5 & 1 & 2,5 \\
\hline Interviennent trop & 5 & 13,5 & 1 & 2,5 \\
\hline Frappent et tapent & 5 & 13,5 & 1 & 2,5 \\
\hline Poussent & 4 & 11,0 & & \\
\hline Retiennent par le bras & 3 & 8,0 & & \\
\hline Punissent de manière abusive & 1 & 2,5 & 2 & 5,5 \\
\hline $\begin{array}{l}\text { Interviennent seulement } \\
\text { s'ils sont à bout }\end{array}$ & 1 & 2,5 & 1 & 2,5 \\
\hline $\begin{array}{l}\text { Mettent l'enfant à genoux ou } \\
\text { dans un coin }\end{array}$ & 1 & 2,5 & & \\
\hline Met du savon dans la bouche & 1 & 2,5 & & \\
\hline A une discipline psychologiquement \\
traumatisante
\end{tabular}

Par ailleurs, quatre parents déclarent que leur conjoint était lui-même violent et que cette situation a laissé des séquelles à leur enfant: Quand je restais avec leur père, il y avait beaucoup de violence, mais c'était plutôt verbal. Il y avait beaucoup de violence verbale, constamment. Puis, des fois, aussi, quand ça faisait pas son affaire, il se fâchait contre des objets. Les enfants ont vu ça (106).

Dans certains cas, il peut s'agir de violence conjugale. À cet effet, une mère raconte que son ex-mari était très agressif, qu'il a proféré des menaces de mort à son endroit et à celui de son nouveau conjoint, que sa petite fille a entendues: Quand je rencontrais mon mari, il disait toujours 
qu'il était pour tuer mon "chum». Il disait ça à mon enfant. Qu'il était pour le battre. Puis, de faire attention parce que s'il nous voyait dans la rue, il était pour nous faire mal (107).

\subsubsection{Le peu ou l'absence de relations avec le père}

Six mères associent les comportements violents de leur enfant au peu de relations que le père entretient avec lui, voire à son absence: Récemment, il a passé un mois sans le voir. Il l'a vu la semaine dernière. Et là, je vois mon fils qui devient très triste et agressif (11.1); Si le père restait dans la vie de mon fils, ça serait pas pire. Mais c'est qu'il vient, puis il disparaît pour huit mois. Là, mon fils est agressif parce qu'il est fâché contre son père parce qu'il est parti (86); Il fait rien que dire que son père lui manque. Puis, tout à coup, il se tourne de bord et dit qu'il l'haït son papa (94.1).

L'une de ces mères confie que c'est le travailleur social de l'école qui a associé les comportements violents de son enfant à l'absence du père: En prématernelle, le travailleur social avait identifié que son problème était l'absence de son père. Des fois, il passait trois mois sans le voir (74).

Par ailleurs, les comportements violents semblent exacerbés lorsque les enfants reviennent d'une visite ou d'une période de garde assumée par le père: C'est un gros problème quand il revient de chez son père. Là, il n'est pas parlable. Il m'envoie promener et n'écoute rien de ce que je dis en revenant de là (05); Lorsque mon fils revient de chez son père, il fonctionne mal pendant deux jours (74); Le comportement de mon garçon devient plus «baveux» [arrogant], moins patient et plus agressif lorsqu'il revient de chez son père (83).

Même lorsque le père a la garde de son enfant, il peut se montrer très peu impliqué: Lorsqu'il a les enfants, il ne s'en occupe pas plus que ça (83); Il $n^{\prime} a$ rien donné pour les enfants. Il ne veut jamais les prendre avec lui (105).

\subsubsection{Le manque de temps des parents}

Sept parents affirment manquer de temps pour s'occuper de leur enfant en raison de leur emploi: Je travaille le soir. Donc, c'est difficile (28); Je suis tellement occupée au bureau qu'il n'y a plus rien qui rentre. Des pleines journées de travail, oui. Puis, je suis souvent obligée de travailler tard le soir et les fins de semaine. Alors, des fois, quand tu arrives à la maison, t'es épuisée (106).

Au manque de temps peut également s'ajouter un surcroît de tâches à accomplir: J'aide mes deux fils avec leurs devoirs. Après ça, mon autre garçon de 5 ans veut que je m'occupe de lui, et ma fille de 2 ans, elle nous interrompt toujours. Après ça, le bébé commence à pleurer (94.1). 
Le manque de temps jumelé à un surcroît de tâches occasionnent, par conséquent, un sentiment d'épuisement chez quatre parents de l'échantillon: L'autre jour, je lui ai dit que je rêvais de me casser une jambe, pour être bien pendant deux semaines à l'hôpital (12.1); Je suis épuisée après ma journée. J'ai des maux de dos en plus (95).

La difficulté de concilier le travail avec la vie familiale apparaît encore plus aiguë lorsqu'il s'agit d'un nouvel emploi, puisque cette situation requiert encore plus d'investissement dans le travail, au détriment de la famille: Le soir quand je reviens, je prépare le souper, on mange, on fait les devoirs, on prend la douche et c'est le coucher. J'ai pas le temps de rien faire d'autre (105). Les stresseurs familiaux affectant négativement les comportements des 6 à 9 ans sont présentés au tableau 3.35.

TABLEAU 3.35

Les stresseurs familiaux affectant négativement les comportements des 6 à 9 ans $(n=37)$

\begin{tabular}{lcccc}
\hline & \multicolumn{2}{c}{ Garçons } & \multicolumn{2}{c}{ Filles } \\
\cline { 2 - 5 } & $N$ & $\%$ & $N$ & $\%$ \\
\cline { 2 - 5 } & 34 & 92,0 & 3 & 8,0 \\
\hline Conflits intrafamiliaux & 13 & 35,0 & 1 & 2,5 \\
\hline Manque de temps des parents & 6 & 16,0 & 1 & 2,5 \\
\hline Absence du père & 6 & 16,0 & & \\
\hline Disputes & 3 & 8,0 & 1 & 2,5 \\
\hline Séparation ou divorce & 3 & 8,0 & 1 & 2,5 \\
\hline Violence conjugale & 3 & 8,0 & 1 & 2,5 \\
\hline Peu de relations avec le père & 3 & 8,0 & 1 & 2,5 \\
\hline $\begin{array}{l}\text { Perturbations au retour } \\
\text { de la garde }\end{array}$ & 3 & 8,0 & & \\
\hline Surcroît de tâches à accomplir & 3 & 8,0 & 1 & 2,5 \\
\hline Épuisement & 3 & 8,0 & 1 & 2,5 \\
\hline $\begin{array}{l}\text { Difficulté à concilier travail } \\
\text { tt famille }\end{array}$ & 3 & 8,0 & 1 & 2,5 \\
\hline Manque d'implication du père & 2 & 5,5 & & \\
\hline
\end{tabular}




\subsection{LES FACTEURS EXPLICATIFS DE LA SITUATION-PROBLÈME À L'ÉCOLE}

Selon les parents de ce sous-échantillon, les facteurs pouvant expliquer la situation-problème de leur enfant à l'école laissent supposer que la structure et le fonctionnement institutionnels représentent des facteurs importants pouvant avoir des répercussions néfastes sur la vie de l'enfant.

\subsubsection{Le changement de classe, d'école ou de personnel scolaire}

Quatre parents mentionnent que le changement de classe, d'école ou de personnel scolaire occasionnent des effets négatifs chez leur enfant: Il a souvent changé d'enseignante depuis le début de l'année scolaire, ce qui cause un autre impact négatif (22); Il a changé d'enseignante pendant l'année et l'adaptation a été difficile (37); En plus, ils ont déménagé d'école. Donc, il y avait plein de bouleversements. En $2^{e}$ année, ils ont refait les classes. Ils ont mis les élèves normaux ensemble et les autres dans une autre classe. Ça allait mal. Lors du changement d'enseignante, ça a pris un mois avant que la structure soit en place, avant de retrouver le moral. Ses enseignantes n'avaient jamais le même style (38).

\subsubsection{Les relations entre l'enseignant et les enfants}

Six parents constatent l'effet négatif que peut avoir une relation difficile entre l'enseignant et leur enfant: Son enseignante lui disait tout le temps qu'il n'était pas capable de rien faire (52); C'est très clair que mon fils et son enseignante, ça ne va pas. Je pense qu'il y a une incompatibilité de caractère entre ces deux-là (99); L'enseignante l'avait humiliée en classe en lui disant en avant de tous les autres qu'elle ne pouvait pas lire, puis qu'elle retardait tout le monde. Ça l'a pas aidée non plus (106).

\subsubsection{La motivation scolaire}

Pour trois parents, l'école impose trop de devoirs et de la matière trop difficile à absorber pour leur enfant: Comme je trouve qu'il y a une chose... une grosse affaire, c'est qu'il y a trop de devoirs. Puis, c'est ça qui cause de la "chicane» [des querelles] plus qu'autre chose (05); L'enfant trouve l'école trop difficile. Donc, ça le décourage (74).

À l'inverse, un autre parent constate que son enfant n'a pas de défi intellectuel à l'école, ce qui influence négativement ses comportements en classe: Il s'ennuie. C'est «plate» [ennuyant] à l'école. Il haït ça. Ce n'est pas intéressant pour lui (88). 
Deux derniers parents affirment que leur enfant n'est pas assez stimulé au plan kinesthésique pour être motivé dans les apprentissages scolaires: En première année, il a eu une enseignante... c'était d'être assis à son pupitre et d'écouter pendant des heures interminables (38).

\subsubsection{La supervision à l'école}

Deux parents affirment qu'ils ne veulent plus envoyer leur enfant à la garderie scolaire en raison d'un manque de supervision: C'était un gros groupe, avec plus ou moins de surveillance. Donc, aussitôt que c'est comme ça, c'est pire. Je peux pas l'envoyer là. Il faut qu'il soit gardé dans une maison (05); Mon enfant se fait toujours punir et ça le fâche puisqu'il sait qu'il y a d'autres enfants qui ont de mauvais comportements et qui ne subissent aucune conséquence parce qu'ils échappent au contrôle de l'enseignante (23). Les facteurs explicatifs de la situation-problème à l'école chez les 6 à 9 ans sont présentés au tableau 3.36 .

TABLEAU 3.36

Les facteurs explicatifs de la situation-problème à l'école chez les 6 à 9 ans $(n=37)$

\begin{tabular}{lcccc}
\hline & \multicolumn{3}{c}{ Garçons } & \multicolumn{2}{c}{ Filles } \\
\cline { 2 - 5 } & $N$ & $\%$ & $N$ & $\%$ \\
\cline { 2 - 5 } & 34 & 92,0 & 3 & 8,0 \\
\hline $\begin{array}{l}\text { Relation enseignant-enfant } \\
\text { difficile }\end{array}$ & 5 & 13,5 & 1 & 2,5 \\
\hline $\begin{array}{l}\text { Changement de classe } \\
\text { ou d'école }\end{array}$ & 4 & 11,0 & & \\
\hline $\begin{array}{l}\text { Changement de personnel } \\
\text { scolaire }\end{array}$ & 4 & 11,0 & \\
\hline Trop de devoirs / Matière difficile & 3 & 8,0 & & \\
\hline $\begin{array}{l}\text { Manque de stimulation } \\
\text { kinesthésique }\end{array}$ & 2 & 5,5 & \\
\hline $\begin{array}{l}\text { Manque de supervision } \\
\text { (récréation) }\end{array}$ & 2 & 5,5 & \\
\hline Absence de défi intellectuel & 1 & 2,5 & \\
\hline
\end{tabular}




\section{LE BILAN DU PORTRAIT DE L'ENSEMBLE DES PARTICIPANTS DE LA RECHERCHE}

La quatrième partie de ce chapitre présente le bilan du portrait de l'ensemble des enfants ayant des comportements violents selon les perceptions parentales et celui des parents ayant participé à la recherche. Ce bilan présente également les principaux faits saillants qui se dégagent du portrait des garçons de 3 à 6 ans et de leurs parents, comparativement à ceux des 6 à 9 ans et de leurs parents. Un regard est également posé sur le portrait des comportements violents chez les garçons comparativement à celui chez les filles de 3 à 9 ans.

\subsection{Les COMPORTEMENTS VIOLENTS des 3 À 9 ANS}

Dans cette partie, nous abordons les comportements de violence physique et verbale, avec ou sans gestes, les problèmes internalisés et externalisés, les problèmes de socialisation, la durée, la fréquence, le lieu et le moment des comportements violents, de même que la trajectoire qu'ils empruntent. Nous terminons par les pratiques éducatives parentales ayant un effet positif et négatif chez les enfants, les stresseurs familiaux qu'ils affrontent et les facteurs explicatifs de la situation-problème à l'école, selon les perceptions des parents de l'ensemble de l'échantillon.

\subsubsection{La violence physique}

Parmi les points saillants qui se dégagent de ce portrait, la violence physique est bien présente chez les enfants de l'échantillon. À cet effet, trois enfants sur quatre frappent et tapent leurs pairs et la fratrie alors que les deux tiers d'entre eux donnent des coups de pied. De même, trois enfants sur cinq donnent des coups de poing ou se battent, tandis que la moitié d'entre eux mordent. Finalement, deux enfants sur cinq malmènent ou intimident leurs pairs, alors que le quart d'entre eux poussent les autres.

Les garçons de 3 à 6 ans sont portés à frapper et à taper les pairs et la fratrie, de même qu'à donner des coups de pied, davantage que ceux de 6 à 9 ans. Également, pousser est un comportement beaucoup plus répandu chez les enfants plus jeunes que chez les plus vieux. Ce dernier comportement est aussi davantage présent chez les filles de l'échantillon que chez les garçons. Par ailleurs, frapper un enseignant ou les parents ne sont des comportements présents que chez les garçons de 3 à 6 ans, tandis que celui de couper les cheveux n'est présent que chez une seule fille du même groupe d'âge. Les comportements de violence physique des 3 à 9 ans sont présentés au tableau 3.37. 
TABLEAU 3.37

Les comportements de violence physique des 3 à 9 ans $(n=60)$

\begin{tabular}{lcccc}
\hline & \multicolumn{2}{c}{ Garçons } & \multicolumn{2}{c}{ Filles } \\
\cline { 2 - 5 } & $\boldsymbol{N}$ & $\%$ & $\boldsymbol{N}$ & $\%$ \\
\cline { 2 - 5 } & 54 & 90,0 & 6 & 10,0 \\
\hline Frappent et tapent & 44 & 73,0 & 1 & 1,5 \\
\hline Donnent des coups de pied & 39 & 65,0 & 2 & 3,5 \\
\hline Donnent des coups de poing & 35 & 58,0 & 2 & 3,5 \\
\hline Se battent & 35 & 58,0 & 1 & 1,5 \\
\hline Mordent & 31 & 51,5 & 1 & 1,5 \\
\hline Malmènent ou intimident & 24 & 40,0 & 1 & 1,5 \\
\hline Poussent & 13 & 21,5 & 3 & 5,0 \\
\hline Frappent l'enseignant & 5 & 22,0 & & \\
\hline Frappent les parents & 3 & 13,0 & & \\
\hline Coupe les cheveux & \multicolumn{5}{c}{1} & 1,5 \\
\hline
\end{tabular}

\subsubsection{La violence verbale avec ou sans gestes}

La violence verbale avec gestes est bien présente chez les enfants de l'échantillon. Les deux tiers d'entre eux détruisent leurs jouets, ceux des autres, ou même d'autres objets qui leur tombent sous la main, tandis que le tiers des enfants frappent des objets, les lancent comme des projectiles, ou même leur donnent des coups de pied.

Quant à la violence verbale sans geste, près de la moitié des enfants insultent et blâment les autres. De plus, deux sur cinq font des menaces. Finalement, le quart des enfants utilisent un langage vulgaire pouvant porter atteinte à l'intégrité des personnes visées.

Les garçons de 3 à 6 ans sont portés à frapper des objets, à les lancer ou à leur donner des coups de pied, davantage que ceux de 6 à 9 ans. Lancer des objets est un comportement davantage présent chez les filles de l'échantillon que chez les garçons. Par ailleurs, blâmer les autres et les insulter sont des comportements beaucoup plus répandus chez les enfants plus vieux que chez les plus jeunes. Ces deux derniers comportements sont également davantage présents chez les filles de l'échantillon que chez les garçons. Les comportements de violence verbale avec ou sans gestes des 3 à 9 ans sont présentés au tableau 3.38 . 
TABLEAU 3.38

Les comportements de violence verbale avec ou sans gestes des 3 à 9 ans $(n=60)$

\begin{tabular}{lcccc}
\hline & \multicolumn{2}{c}{ Garçons } & \multicolumn{2}{c}{ Filles } \\
\cline { 2 - 5 } & $\boldsymbol{N}$ & $\%$ & $\boldsymbol{N}$ & $\%$ \\
\cline { 2 - 5 } & 54 & 90,0 & 6 & 10,0 \\
\hline Détruisent des objets & 35 & 58,0 & 3 & 5,0 \\
\hline Insultent & 33 & 55,0 & 4 & 6,5 \\
\hline Blâment & 32 & 53,0 & 5 & 8,0 \\
\hline Font des menaces & 23 & 38,0 & 2 & 3,5 \\
\hline Frappent des objets & 19 & 31,5 & 2 & 3,5 \\
\hline Lancent des objets & 18 & 30,0 & 3 & 5,0 \\
\hline Donnent des coups de pied & 18 & 30,0 & 2 & 3,5 \\
aux objets & 13 & 21,5 & 2 & 3,5 \\
\hline Utilisent un langage vulgaire & 13
\end{tabular}

\subsubsection{Les problèmes internalisés}

L'anxiété et l'insécurité ressortent chez plus des trois quarts des enfants de l'échantillon. Les sentiments dépressifs sont également présents chez plus de la moitié d'entre eux. De plus, un peu moins de la moitié des enfants manquent d'estime de soi, tandis que le tiers manquent de confiance en soi. Finalement, un enfant sur six s'isole et quelques-uns se blâment ou entretiennent des idées suicidaires.

Les sentiments dépressifs, le manque d'estime de soi et le manque de confiance en soi sont davantage présents chez les garçons de 3 à 6 ans que chez ceux de 6 à 9 ans. Ces deux dernières caractéristiques, de même que l'anxiété et l'isolement, sont davantage présents chez les filles de l'échantillon que chez les garçons. Par ailleurs, l'anxiété et l'insécurité sont des problèmes davantage présents chez les enfants plus vieux que chez les plus jeunes. Finalement, entretenir des idées suicidaires n'est un problème présent que chez les garçons de l'échantillon, tandis que celui de poser des gestes de violence physique contre soi n'est présent que chez une seule fille de 6 à 9 ans. Les problèmes internalisés des 3 à 9 ans sont présentés au tableau 3.39 . 
TABLEAU 3.39

Les problèmes internalisés des 3 à 9 ans $(n=60)$

\begin{tabular}{lcccc}
\hline & \multicolumn{2}{c}{ Garçons } & \multicolumn{2}{c}{ Filles } \\
\cline { 2 - 5 } & $N$ & $\%$ & $N$ & $\%$ \\
\cline { 2 - 5 } & 54 & 90,0 & 6 & 10,0 \\
\hline Éprouvent de l'anxiété & 45 & 75,0 & 3 & 5,0 \\
\hline Se sentent insécures & 45 & 75,0 & 2 & 3,5 \\
\hline Ont des sentiments dépressifs & 32 & 53,0 & 1 & 1,5 \\
\hline Manquent d'estime de soi & 23 & 38,0 & 5 & 8,0 \\
\hline Manquent de confiance en soi & 19 & 31,5 & 4 & 6,5 \\
\hline S'isolent & 8 & 13,0 & 2 & 3,5 \\
\hline Se blâment & 4 & 6,5 & 1 & 1,5 \\
\hline Entretiennent des idées suicidaires & 3 & 5,0 & & \\
\hline Pose des gestes contre soi & & & 1 & 1,5 \\
\hline
\end{tabular}

\subsubsection{Les problèmes externalisés}

La presque totalité des enfants s'opposent à l'adulte et n'écoutent pas les consignes et les trois quarts d'entre eux chialent. Par ailleurs, les deux tiers des enfants sont aux prises avec de la suractivité, des sautes d'humeur et de l'impulsivité, alors qu'un peu moins de la moitié des enfants font des crises et ressentent de la frustration. De plus, le tiers d'entre eux se laissent influencer négativement par les pairs alors qu'un peu moins du quart font des colères. Enfin, un enfant sur six fait preuve d'impatience.

S'opposer à l'adulte, ne pas écouter les consignes, «chialer», [se plaindre], être aux prises avec de la suractivité, des sautes d'humeur, de l'impulsivité et de la frustration sont des problèmes davantage présents chez les garçons de 6 à 9 ans que chez ceux de 3 à 6 ans. Ne pas écouter les consignes et chialer sont également davantage présents chez les filles de l'échantillon que chez les garçons. Par ailleurs, se faire influencer négativement par les pairs et refuser de se soumettre à l'autorité sont des problèmes uniquement présents chez les enfants plus vieux, tandis que manquer de contrôle de soi, mentir, voler, fuguer et tricher sont uniquement présents chez les garçons de 6 à 9 ans. Les problèmes externalisés des 3 à 9 ans sont présentés au tableau 3.40 . 
Tableau 3.40

Les problèmes externalisés des 3 à 9 ans $(n=60)$

\begin{tabular}{lcccr}
\hline & \multicolumn{2}{c}{ Garçons } & \multicolumn{2}{c}{ Filles } \\
\cline { 2 - 5 } & $\boldsymbol{N}$ & $\%$ & $\boldsymbol{N}$ & $\%$ \\
\cline { 2 - 5 } & 54 & 90,0 & 6 & 10,0 \\
\hline S'opposent à l'adulte & 48 & 80,0 & 5 & 8,0 \\
\hline N'écoutent pas les consignes & 46 & 76,5 & 6 & 10,0 \\
\hline «Chialent» (se plaignent) & 40 & 66,5 & 5 & 8,0 \\
\hline Font preuve de suractivité & 40 & 66,5 & 2 & 3,5 \\
\hline Ont des changements d'humeur & 39 & 65,0 & 2 & 3,5 \\
\hline Font preuve d'impulsivité & 36 & 60,0 & 3 & 5,0 \\
\hline Font des crises & 24 & 40,0 & 2 & 3,5 \\
\hline Éprouvent de la frustration & 23 & 38,0 & 2 & 3,5 \\
\hline Se font influencer & 21 & 35,0 & 1 & 1,5 \\
\hline Font des colères & 13 & 21,5 & 1 & 1,5 \\
\hline Font preuve d'impatience & 8 & 13,0 & 2 & 3,5 \\
\hline Manquent de contrôle de soi & 5 & 13,5 & & \\
\hline Mentent & 5 & 13,5 & & \\
\hline Volent & 5 & 13,5 & & \\
\hline Fuguent & 2 & 3,5 & & \\
\hline Triche & 1 & 1,5 & & \\
\hline
\end{tabular}

\subsubsection{Les problèmes de socialisation}

La moitié des enfants éprouvent de grandes difficultés à fonctionner en groupe. C'est pourquoi ils s'isolent et jouent beaucoup plus souvent seuls qu' avec les autres. De plus, le tiers d'entre eux ont de la difficulté à partager, tandis que le quart des enfants refusent de se plier aux règles. Par ailleurs, un enfant sur six se fait pousser, est l'objet de moqueries ou se fait frapper et taper par des pairs. Finalement, quelques-uns se sentent rejetés et un dernier garçon se fait souvent agacer par les autres.

Les enfants de 3 à 6 ans éprouvent davantage de difficulté à fonctionner en groupe que les 6 à 9 ans. Ce problème est également davantage présent chez les filles de l'échantillon que chez les garçons. Également, les plus jeunes enfants sont davantage que les plus vieux portés à s'isoler, à jouer seuls et à éprouver de la difficulté à partager. Par ailleurs, refuser de se plier aux règles est un problème uniquement présent chez les enfants 
de 3 à 6 ans tandis que se sentir rejeté et se faire agacer ne se rencontrent que chez les garçons de 6 à 9 ans. Les problèmes de socialisation des 3 à 9 ans sont présentés au tableau 3.41.

TABLEAU 3.41

Les problèmes de socialisation des 3 à 9 ans $(n=60)$

\begin{tabular}{lcccc}
\hline & \multicolumn{2}{c}{ Garçons } & \multicolumn{2}{c}{ Filles } \\
\cline { 2 - 5 } & $\boldsymbol{N}$ & $\%$ & $\boldsymbol{N}$ & $\%$ \\
\cline { 2 - 5 } & 54 & 90,0 & 6 & 10,0 \\
\hline Jouent seuls & 27 & 45,0 & 2 & 3,5 \\
\hline Ne fonctionnent pas en groupe & 24 & 40,0 & 4 & 6,5 \\
\hline S'isolent & 23 & 38,0 & 1 & 1,5 \\
\hline Ont de la difficulté à partager & 17 & 28,0 & 2 & 3,5 \\
\hline Refusent de se plier aux règles & 11 & 18,0 & 2 & 3,5 \\
\hline Se font pousser par les autres & 9 & 15,0 & 1 & 1,5 \\
\hline Sont l'objet de moqueries & 8 & 13,0 & 1 & 1,5 \\
\hline Se font frapper et taper & 8 & 13,0 & 1 & 1,5 \\
\hline Se sentent rejetés & 2 & 3,5 & & \\
\hline Se fait agacer & 1 & 1,5 & & \\
\hline
\end{tabular}

\subsection{LA DURÉE ET LA FRÉQUENCE DES COMPORTEMENTS VIOLENTS}

En ce qui a trait à la durée des comportements violents, selon les perceptions parentales, il semble difficile de pouvoir fixer précisément depuis combien de temps ils sont présents, tant chez les enfants de 3 à 6 ans que chez ceux de 6 à 9 ans. Quoi qu'il en soit, les parents déclarent également que ces comportements durent depuis une certaine période de temps, variant de six mois à trois ans et demi.

En ce qui concerne la fréquence des comportements violents, chez un peu moins du quart des enfants, ils surviennent une fois par jour, tandis que chez un autre quart, ils se produisent plusieurs fois par semaine. Chez un enfant sur cinq, ils sont présents une fois par semaine, tandis que pour un peu moins d'un enfant sur six, ces comportements se produisent à raison de plusieurs fois par mois. Chez un enfant sur dix, ces comportements surviennent une fois par mois, alors que chez quelques enfants, ils se produisent de manière épisodique. 
En général, les comportements violents sont beaucoup plus fréquents chez les garçons de 3 à 6 ans que chez ceux de 6 à 9 ans. Également, ils surviennent beaucoup plus fréquemment chez les garçons de l'échantillon que chez les filles. La fréquence des comportements violents des 3 à 9 ans est présentée au tableau 3.42 .

TABleau 3.42

La fréquence des comportements violents des 3 à 9 ans $(n=60)$

\begin{tabular}{lcccc}
\hline & \multicolumn{2}{c}{ Garçons } & \multicolumn{2}{c}{ Filles } \\
\cline { 2 - 5 } & $\boldsymbol{N}$ & $\%$ & $\boldsymbol{N}$ & $\%$ \\
\cline { 2 - 5 } & 54 & 90,0 & 6 & 10,0 \\
\hline Une fois par jour & 12 & 20,0 & & \\
\hline Plusieurs fois par semaine & 14 & 23,0 & 1 & 1,5 \\
\hline Une fois par semaine & 12 & 20,0 & & \\
\hline Plusieurs fois par mois & 5 & 13,5 & 3 & 5,0 \\
\hline Une fois par mois & 6 & 10,0 & & \\
\hline De manière épisodique & 5 & 13,5 & 2 & 3,5 \\
\hline
\end{tabular}

\subsection{LE LIEU ET LE MOMENT DES COMPORTEMENTS VIOLENTS}

En ce qui a trait au lieu des comportements violents, plus des trois quarts des parents déclarent qu'ils surviennent davantage à l'école qu'à la maison. Quant au moment où ils se produisent, le tiers d'entre eux identifient que c'est surtout lors des récréations, lors du dîner et à la fin de la journée scolaire, lorsque la fatigue est plus grande. Par ailleurs, chez le quart des parents qui déclarent que les comportements violents sont davantage présents à la maison qu'à l'école, ils identifient qu'ils surviennent plus précisément le matin, lors de la période des devoirs et le soir.

L'école semble davantage le lieu où s'exerce la violence chez les garçons de 3 à 6 ans que chez ceux de 6 à 9 ans. C'est également à l'école que les comportements violents des filles de l'échantillon surviennent majoritairement. De plus, chez les jeunes garçons, ils se produisent davantage à la fin de l'après-midi qu'à un autre moment de la journée. Cette situation est peut-être attribuable au fait que leur fatigue est plus grande à ce moment précis de la journée. Chez les plus vieux, les comportements violents surviennent plus précisément lors des récréations et de la période du dîner. C'est également lors de ces deux moments, en plus de la fin de l'après-midi, que s'exercent les comportements violents chez les filles de l'échantillon. Par ailleurs, les comportements violents qui surviennent à 
la maison, que ce soit le matin, lors des devoirs ou le soir, ne concernent que des garçons de 6 à 9 ans. Le lieu et le moment des comportements violents des 3 à 9 ans sont présentés au tableau 3.43.

TABLEAU 3.43

Le lieu et le moment des comportements violents des 3 à 9 ans $(n=60)$

\begin{tabular}{|c|c|c|c|c|}
\hline & \multicolumn{2}{|c|}{ Garçons } & \multicolumn{2}{|c|}{ Filles } \\
\hline & $N$ & $\%$ & $N$ & $\%$ \\
\hline & 54 & 90,0 & 6 & 10,0 \\
\hline À l'école & 42 & 70,0 & 5 & 8,0 \\
\hline Lors des récréations & 7 & 11,5 & 2 & 3,5 \\
\hline Lors du dîner & 4 & 6,5 & 1 & 1,5 \\
\hline À la fin de la journée & 7 & 11,5 & 2 & 3,5 \\
\hline À la maison & 12 & 20,0 & 1 & 1,5 \\
\hline Le matin & 3 & 5,0 & & \\
\hline Lors des devoirs & 2 & 3,5 & & \\
\hline Le soir & 2 & 3,5 & & \\
\hline
\end{tabular}

\subsection{LA TRAJECTOIRE DES COMPORTEMENTS VIOLENTS}

Un peu moins du quart des parents reconnaissent qu'ils ont coïncidé avec l'entrée en maternelle/jardin, tandis que le tiers d'entre eux déclarent que c'est avec l'entrée en première année. Quelques autres évoquent une période bien antérieure au début du préscolaire ou du primaire. Pour ces derniers, les comportements ont empiré depuis l'entrée en garderie ou en première année. Finalement, un parent sur cinq déclare que les comportements violents de son enfant se sont améliorés depuis l'entrée au primaire.

L'entrée en maternelle/jardin ainsi qu'en première année sont des périodes qui coïncident davantage que d'autres avec l'émergence des comportements violents, tant chez les garçons de l'échantillon que chez les filles. Toutefois, ces comportements ont davantage empiré depuis la période préscolaire que durant celle de la scolarisation. À cet effet, seuls des parents d'enfants de 6 à 9 ans mentionnent que les comportements violents de leur enfant se sont améliorés depuis le début du primaire. Par ailleurs, chez les filles de l'échantillon, aucun comportement violent n'a coïncidé avec l'entrée en garderie, avant l'entrée au primaire et avec 
l'entrée en troisième année, et ceux-ci ne se sont pas empirés depuis leur première année. La trajectoire des comportements violents chez les 3 à 9 ans est présentée au tableau 3.44.

TABLeau 3.44

La trajectoire des comportements violents chez les 3 à 9 ans $(n=60)$

\begin{tabular}{lrrrr}
\hline & \multicolumn{2}{c}{ Garçons } & \multicolumn{2}{c}{ Filles } \\
\cline { 2 - 5 } & $\boldsymbol{N}$ & $\%$ & $\boldsymbol{N}$ & $\%$ \\
\cline { 2 - 5 } & 54 & 90,0 & 6 & 10,0 \\
\hline Avec l'entrée en maternelle/jardin & 11 & 18,0 & 1 & 1,5 \\
\hline Avec l'entrée à la garderie & 6 & 10,0 & & \\
\hline Avant l'entrée au préscolaire & 3 & 5,0 & 1 & 1,5 \\
\hline Pires depuis le préscolaire & 8 & 13,0 & 1 & 1,5 \\
\hline Avec l'entrée en première année & 21 & 35,0 & 2 & 3,5 \\
\hline Avec l'entrée en deuxième année & 5 & 8,0 & 1 & 1,5 \\
\hline Avec l'entrée en troisième année & 5 & 8,0 & & \\
\hline Avant l'entrée au primaire & 3 & 5,0 & & \\
\hline Pires depuis la première année & 3 & 5,0 & & \\
\hline Mieux aujourd'hui & 10 & 16,5 & 1 & 1,5 \\
\hline
\end{tabular}

\subsection{LES PRATIQUES ÉDUCATIVES PARENTALES AYANT UN EFFET POSITIF}

$\mathrm{Au}$ plan des pratiques éducatives parentales ayant un effet positif chez leur enfant à la maison, les deux tiers des parents mentionnent recourir au dialogue et au retrait. Ces parents mentionnent aussi qu'ils utilisent toujours la même technique. Également, un peu plus de la moitié d'entre eux utilisent la perte de privilèges tandis qu'un peu moins de la moitié ont recours au renforcement positif. Le quart des parents ont recours à la technique 1-2-3. Finalement, un parent sur six déclare maintenir une routine que l'enfant doit suivre et quelques-uns ont recours à des tâches supplémentaires.

Le dialogue est davantage utilisé par les parents des garçons de 6 à 9 ans que chez ceux de 3 à 6 ans. De même, les parents des garçons les plus vieux déclarent davantage que ceux des plus jeunes utiliser toujours la même technique. Également, les parents d'enfants de 3 à 6 ans ont davantage recours au renforcement positif que chez ceux de 6 à 9 ans. Par ailleurs, les parents des filles de l'échantillon utilisent davantage le 
retrait, la perte de privilèges, le renforcement positif et la technique 1-2-3, de même que le recours au maintien d'une routine que les parents des garçons. Enfin, seuls les parents d'enfants de 6 à 9 ans de l'échantillon disent utiliser des tâches supplémentaires. Le recours à ces tâches est toutefois davantage l'affaire des parents de ces garçons que de ceux des filles. Finalement, aucun parent des garçons de 6 à 9 ans n'a recours au maintien d'une routine et aucun parent des filles du même âge n'utilise la technique 1-2-3. Les pratiques éducatives parentales ayant un effet positif chez les 3 à 9 ans selon les parents sont présentées au tableau 3.45.

TABLEAU 3.45

Les pratiques éducatives parentales ayant un effet positif chez les 3 à 9 ans $(n=60)$

\begin{tabular}{lcccc}
\hline & \multicolumn{2}{c}{ Garçons } & \multicolumn{2}{c}{ Filles } \\
\cline { 2 - 5 } & $\boldsymbol{N}$ & $\%$ & $\boldsymbol{N}$ & $\%$ \\
\cline { 2 - 5 } & 54 & 90,0 & 6 & 10,0 \\
\hline Dialogue & 37 & 61,5 & 4 & 6,5 \\
\hline Toujours la même technique & 37 & 61,5 & 3 & 5,0 \\
\hline Retrait & 36 & 60,0 & 5 & 8,0 \\
\hline Perte de privilèges & 30 & 50,0 & 4 & 6,5 \\
\hline Renforcement positif & 26 & 43,0 & 4 & 6,5 \\
\hline Technique 1-2-3 & 14 & 23,0 & 2 & 3,5 \\
\hline Maintien d'une routine & 8 & 13,0 & 2 & 3,5 \\
\hline Tâches supplémentaires & 3 & 5,0 & 1 & 1,5 \\
\hline
\end{tabular}

\subsection{LES PRATIQUES ÉDUCATIVES PARENTALES AYANT UN EFFET NÉGATIF}

Concernant les pratiques éducatives parentales ayant un effet négatif chez leur enfant à la maison, la moitié des parents avouent ne pas toujours intervenir, surtout lorsqu'ils sont fatigués. Un autre tiers d'entre eux crient après leur enfant tandis que la moitié des parents déclarent trop intervenir. De plus, un parent sur cinq admet ne pas avoir de discipline assez stricte, punir de manière abusive ou frapper et taper son enfant. Finalement, chez une minorité d'entre eux, le parent intervient seulement lorsqu'il est à bout, met son enfant à genoux dans un coin, le gâte trop, manque de structure, met du savon dans la bouche, exerce une discipline traumatisante psychologiquement ou frappe des objets devant lui. 
Les parents des garçons de 3 à 6 ans déclarent davantage punir leur enfant de manière abusive que ceux de 6 à 9 ans. De même, les parents des enfants les plus vieux déclarent davantage qu'ils n'ont pas de discipline assez stricte que ceux des plus jeunes. Quant aux parents des filles de l'échantillon, ils admettent davantage que les parents des garçons crier après leur enfant, trop intervenir, la frapper et la taper. Par ailleurs, seuls les parents d'enfants de 6 à 9 ans admettent avoir poussé leur enfant. Également, seuls les parents des garçons de 3 à 9 ans déclarent ne pas avoir de discipline assez stricte et seuls ceux des garçons de 3 à 6 ans déclarent trop gâter leur enfant et manquer de structure. De plus, seuls les parents des garçons de 6 à 9 ans déclarent le retenir par le bras, le punir de manière abusive, lui mettre du savon dans la bouche, exercer une discipline traumatisante psychologiquement et frapper des objets devant lui. Les pratiques éducatives parentales ayant un effet négatif chez les 3 à 9 ans selon les parents sont présentées au tableau 3.46.

TABleau 3.46

Les pratiques éducatives parentales ayant un effet négatif chez les 3 à 9 ans $(n=60)$

\begin{tabular}{lcccc}
\hline & \multicolumn{2}{c}{ Garçons } & \multicolumn{2}{c}{ Filles } \\
\cline { 2 - 5 } & $N$ & $\%$ & $N$ & $\%$ \\
\cline { 2 - 5 } & 54 & 90,0 & 6 & 10,0 \\
\hline N'interviennent pas toujours & 28 & 46,5 & 3 & 5,0 \\
\hline Crient & 20 & 33,0 & 3 & 5,0 \\
\hline Interviennent trop & 14 & 23,0 & 2 & 3,5 \\
\hline N'ont pas de discipline & 11 & 18,0 & & \\
assez stricte & 10 & 16,5 & 1 & 1,5 \\
\hline Punissent de manière abusive & 8 & 13,0 & 2 & 3,5 \\
\hline Frappent et tapent & 5 & 8,0 & 1 & 1,5 \\
\hline Poussent & 1 & 1,5 & 2 & 3,5 \\
\hline Interviennent seulement s'ils & 1 & 1,5 & 1 & 1,5 \\
\hline sont à bout & 1 & 1,5 & & \\
\hline Mettent l'enfant à genoux & 1 & 1,5 & & \\
\hline dans un coin & 1 & 1,5 & & \\
\hline Gâte l'enfant & 1 & 1,5 & & \\
\hline Manque de structure & 1 & 1,5 & & \\
\hline Met du savon dans la bouche & & & \\
\hline $\begin{array}{l}\text { A une discipline psychologiquement } \\
\text { traumatisante }\end{array}$ & 1 & & & \\
\hline Frappe des objets & 1 & & \\
\hline
\end{tabular}




\subsection{LES STRESSEURS FAMILIAUX}

En ce qui concerne les stresseurs familiaux ayant un effet négatif sur les comportements de leur enfant, la moitié des parents rapportent que les conflits intrafamiliaux en constituent la cause principale. Dans le même ordre d'idées, un parent sur dix reconnaît que les disputes, la violence conjugale et la séparation ou le divorce sont responsables de tels comportements. Le peu de relations avec le père constitue un autre stresseur familial responsable, selon le quart des parents, de même que son absence, déclarée par un parent sur sept. Quelques autres parents rapportent que les perturbations que vit leur enfant au retour de la période de garde assumée par le père, de même que son manque d'implication ont un effet négatif sur le comportement de l'enfant. Par ailleurs, le tiers des parents considèrent le manque de temps comme un autre stresseur responsable de comportements violents. À ce manque de temps s'ajoute la difficulté de concilier le travail et la vie familiale chez un parent sur cinq. Enfin, quelques autres parents voient dans l'isolement social, le surcroît de tâches à accomplir ou l'épuisement, la cause principale des comportements violents de leur enfant alors que pour une dernière mère, $c^{\prime}$ est la maladie chronique dont elle est atteinte qui en est responsable.

Les parents des garçons de 3 à 6 ans considèrent davantage que ceux des 6 à 9 ans les conflits intrafamiliaux comme la cause principale des comportements violents de leur enfant. De même, selon les parents des plus jeunes davantage que chez ceux des plus vieux, le peu de relations avec le père, le manque de temps ainsi que la difficulté de concilier le travail avec la vie familiale ont un effet négatif sur les comportements de leur enfant. La difficulté de concilier le travail et la famille est davantage présente chez les parents des filles de l'échantillon, que chez ceux des garçons. Par ailleurs, seuls les parents des garçons de l'échantillon voient dans l'absence du père, l'isolement social, les perturbations au retour de la garde chez le père et son manque d'implication, que ces stresseurs familiaux sont susceptibles d'avoir un effet négatif sur les comportements de l'enfant. Les stresseurs familiaux ayant un effet négatif sur les comportements des 3 à 9 ans selon les parents sont présentés au tableau 3.47. 
TABLEAu 3.47

Les stresseurs familiaux affectant négativement les comportements des 3 à 9 ans $(n=60)$

\begin{tabular}{lcccc}
\hline & \multicolumn{2}{c}{ Garçons } & \multicolumn{2}{c}{ Filles } \\
\cline { 2 - 5 } & $N$ & $\%$ & $N$ & $\%$ \\
\cline { 2 - 5 } & 54 & 90,0 & 6 & 10,0 \\
\hline Conflits intrafamiliaux & 27 & 45,0 & 2 & 3,5 \\
\hline Manque de temps des parents & 17 & 28,0 & 2 & 3,5 \\
\hline Peu de relations avec le père & 16 & 26,5 & 1 & 1,5 \\
\hline Difficulté à concilier travail et famille & 9 & 15,0 & 3 & 5,0 \\
\hline Absence du père & 9 & 15,0 & & \\
\hline Disputes & 6 & 10,0 & 1 & 1,5 \\
\hline Violence conjugale & 6 & 10,0 & 1 & 1,5 \\
\hline Isolement social & 5 & 8,0 & & \\
\hline Séparation ou divorce & 4 & 6,5 & 2 & 3,5 \\
\hline Surcroît de tâches à accomplir & 3 & 5,0 & 1 & 1,5 \\
\hline Épuisement & 3 & 5,0 & 1 & 1,5 \\
\hline Perturbations au retour de la garde & 3 & 5,0 & & \\
\hline Manque d'implication du père & 2 & 3,5 & & \\
\hline Maladie de la mère & 1 & 1,5 & & \\
\hline
\end{tabular}

\subsection{LES FACTEURS EXPLICATIFS DE LA SITUATION-PROBLĖME À L'ÉCOLE}

Au plan des facteurs explicatifs de la situation-problème à l'école, le quart des parents trouvent que l'organisation scolaire est trop rigide. De plus, un parent sur six décrit la période de la récréation comme étant la plus propice à l'émergence de comportements violents en raison du manque de supervision. Dans les mêmes proportions, l'enseignant n'accorde pas suffisamment d'attention aux enfants. Par ailleurs, un parent sur six dénonce l'instabilité du personnel scolaire ou les changements de classe ou d'école qui, semble-t-il, contribuent à perturber l'enfant. Les parents citent aussi la gestion de classe inappropriée, l'étiquetage des enfants par le personnel scolaire et la relation difficile entre l'enseignant et l'enfant parmi les facteurs pouvant contribuer à l'émergence des comportements violents chez leur enfant. Finalement, la trop grande charge de devoirs ou la matière trop difficile, l'absence de défi intellectuel, la discipline trop sévère ou trop permissive sont autant de facteurs pouvant avoir un effet négatif sur les comportements des enfants à l'école. 
Les parents des garçons de 3 à 6 ans considèrent davantage que ceux des 6 à 9 ans le manque de supervision durant la récréation et le changement de personnel scolaire comme des facteurs pouvant expliquer les comportements violents de leur enfant à l'école. Seuls les parents des plus jeunes garçons trouvent qu'une organisation scolaire trop rigide, l'étiquetage des enfants, le manque d'attention de la part de l'enseignant, la gestion de classe inadéquate ou une discipline trop sévère ou trop permissive sont des facteurs explicatifs de la situation-problème de leur enfant à l'école. À l'inverse, seuls les parents des garçons de 6 à 9 ans trouvent qu'une relation difficile entre l'enseignant et l'enfant, la trop grande charge de devoirs et la difficulté de la matière, le manque de stimulation kinesthésique ou l'absence de défi intellectuel peuvent expliquer les comportements violents de leur enfant à l'école. Par ailleurs, seuls les parents des garçons de l'échantillon considèrent le manque de supervision durant la récréation, le changement de personnel scolaire, le changement de classe ou d'école, la trop grande charge de devoirs et la difficulté de la matière, le manque de stimulation kinesthésique ou encore la discipline trop sévère ou trop permissive comme des facteurs explicatifs de la situation-problème de leur enfant à l'école. Les facteurs explicatifs de la situation-problème à l'école chez les 3 à 9 ans selon les parents sont présentés au tableau 3.48.

TABLEAU 3.48

Les facteurs explicatifs de la situation-problème à l'école chez les 3 à 9 ans $(n=60)$

\begin{tabular}{lcccc}
\hline & \multicolumn{2}{c}{ Garçons } & \multicolumn{2}{c}{ Filles } \\
\cline { 2 - 5 } & $N$ & $\%$ & $N$ & $\%$ \\
\cline { 2 - 5 } & 54 & 90,0 & 6 & 10,0 \\
\hline Organisation scolaire trop rigide & 13 & 21,5 & 2 & 3,5 \\
\hline Manque de supervision (récréation) & 10 & 16,5 & & \\
\hline Manque d'attention de l'enseignant & 9 & 15,0 & 1 & 1,5 \\
\hline Changement de personnel scolaire & 9 & 15,0 & & \\
\hline Gestion de classe inadéquate & 7 & 11,5 & 1 & 1,5 \\
\hline Étiquetage des enfants & 6 & 10,0 & 2 & 3,5 \\
\hline Relation enseignant-enfant difficile & 5 & 8,0 & 1 & 1,5 \\
\hline Changement de classe ou d'école & 5 & 8,0 & & \\
\hline Trop de devoirs / Matière difficile & 3 & 5,0 & & \\
\hline Discipline scolaire trop sévère & 2 & 3,5 & & \\
\hline Discipline scolaire trop permissive & 2 & 3,5 & & \\
\hline Manque de stimulation kinesthésique & 2 & 3,5 & & \\
\hline
\end{tabular}


En définitive, quels que soient les facteurs invoqués par les parents pour expliquer les comportements violents de leur enfant à l'école, il leur semble important que ce dernier puisse bénéficier d'une attention personnalisée. Il leur paraît également essentiel que le personnel scolaire travaille avec eux de manière concertée afin d'atteindre des objectifs communs concernant l'enfant. Afin d'y parvenir, le meilleur moyen semble d'instaurer une bonne collaboration entre l'école et les parents.

Le prochain chapitre est entièrement consacré à la présentation des résultats portant sur la collaboration entre l'école et les parents d'enfants de 3 à 9 ans ayant des comportements violents envers leurs pairs au préscolaire et au primaire. 



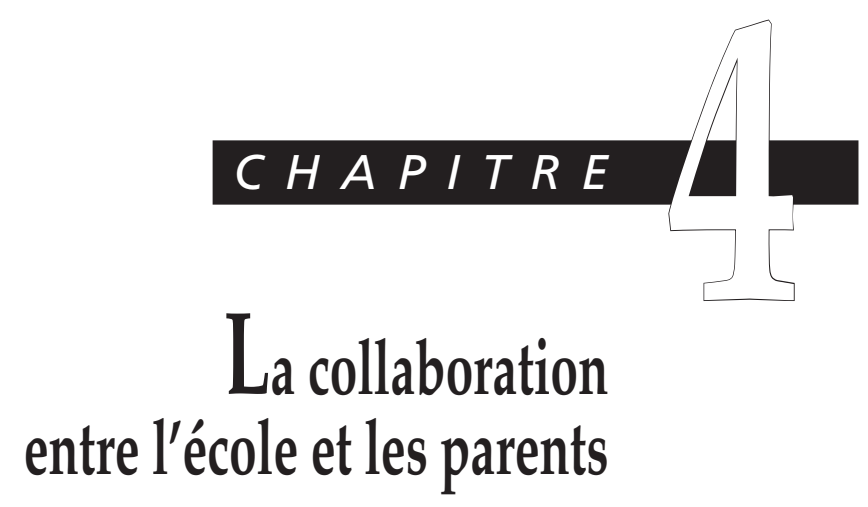



Le quatrième chapitre de cet ouvrage présente l'analyse des résultats portant sur la collaboration entre l'école et les parents d'enfants de 3 à 9 ans ayant des comportements violents envers leurs pairs au préscolaire et au primaire. Cette analyse fait état des perceptions des soixante parents ayant participé à la présente recherche. Dans la première partie, le concept de collaboration fait l'objet d'un rappel théorique. Puis, afin de mieux cerner ce que les parents considèrent comme étant de la collaboration, nous regroupons leurs propos selon deux des sous-concepts qui lui sont étroitement associés, soit l'implication parentale dans la vie scolaire de l'enfant et la communication entre les deux milieux. Dans la deuxième partie, nous abordons un troisième sous-concept, soit celui de la participation au processus de prise de décisions concernant l'enfant, puisqu'il représente un élément central de la collaboration. Puis, les troisième et quatrième parties présentent les facteurs de soutien et les obstacles sous-jacents à la collaboration, en fonction des profils de parents qui collaborent et de ceux qui ne collaborent pas avec l'école. Quant à la cinquième partie, elle aborde d'autres éléments importants de la collaboration à considérer sous l'angle des perceptions que les parents entretiennent face au personnel scolaire et à l'école. Finalement, la sixième partie traite de l'empowerment parental, soit les gestes d'adaptation, d'opposition et de résistance que posent les parents afin d'exercer une plus grande influence, voire un meilleur contrôle sur la situation-problème de leur enfant à l'école.

\section{AU SUJET DU CONCEPT DE COLLABORATION}

Dans la foulée des écrits portant sur la théorie émergente de Straus et Corbin (1990), cette première partie présente les unités de sens qui se dégagent des propos recueillis auprès des parents et les liens que nous établissons avec les principaux écrits publiés sur le sujet ces dernières années. Ces résultats illustrent la complexité de la collaboration entre l'école et les parents dans le cadre de la problématique des comportements violents envers les pairs au préscolaire et au primaire.

Les perceptions des parents soulèvent une diversité de facettes. Ces facettes sont exprimées en fonction des dimensions émergeant du concept de collaboration, fondé sur la définition de Christenson et Sheridan (2001, p. 95), à savoir «un cadre de travail dynamique impliquant des styles $\mathrm{d}$ 'interaction collégiaux, interdépendants et égalitaires entre le personnel scolaire et les parents, les deux parties travaillant à l'atteinte de buts communs». 
Les propos des parents sont également analysés sous l'angle de leur implication dans la vie scolaire de leur enfant et de la communication qu'ils entretiennent avec le personnel scolaire. À cet égard, l'implication parentale dans la vie scolaire de l'enfant, selon la typologie d'Epstein (1995), jette un premier éclairage sur les facteurs de soutien et les obstacles sous-jacents à la collaboration entre l'école et les parents. Une telle collaboration se traduit par un travail complémentaire réalisé à partir de buts communs, qui s'appuie sur une communication bidirectionnelle, enrichissante et productive, ainsi que sur une confiance et un soutien mutuels, tel qu'en font foi Adams et Christenson (2000) dans un article scientifique qui a fait école aux États-Unis.

\subsection{L'IMPLICATION PARENTALE DANS LA VIE SCOLAIRE DE L'ENFANT}

Plus des deux tiers des parents de la présente recherche s'impliquent dans la vie scolaire de leur enfant. Ils le font notamment en participant à des activités de bénévolat, en faisant partie de comités de parents, en assistant leur enfant durant la période de devoirs et de leçons, ou même en leur offrant un soutien parental dans toutes tâches scolaires connexes. Également, certains parents participent à des groupes de parentage afin d'améliorer l'efficacité de leurs interventions à la maison auprès de leur enfant. Cette implication rejoint quatre des six types décrits dans la typologie d'Epstein (1995).

L'analyse des données d'entrevue révèle toutefois des distinctions notables entre l'implication des parents vivant en contexte de pauvreté et ceux des classes moyenne et moyenne-supérieure. Ainsi, les parents s'impliquent de manières différentes dans la vie scolaire de leur enfant. Aux dires de McCarthey (2000), ces différents types d'implication bénéficient tout autant à l'enfant. C'est pourquoi, lorsqu'ils s'avèrent utiles à la compréhension du phénomène, certains résultats du présent chapitre sont présentés en fonction de deux regroupements, soit celui des parents vivant en contexte de pauvreté et celui des parents des classes moyenne et moyenne-supérieure.

\subsubsection{Les activités de bénévolat à l'école}

Seulement trois parents de l'échantillon vivant en contexte de pauvreté s'impliquent dans des activités de bénévolat à l'école: Présentement, je fais du bénévolat à la garderie scolaire (06); Moi, j'essaie d'aider le plus que je peux. Quand ils me demandent de faire quelque chose, si je peux, je vais le faire (112). 
Quant aux parents des classes moyenne et moyenne-supérieure, près du tiers d'entre eux s'impliquent dans de telles activités, faisant suite à l'invitation de l'école. De ce nombre, deux sont des grands-mères qui agissent en remplacement de la mère qui travaille et qui n'a pas le temps d'y participer: Ma mère y va à ma place. Elle s'implique beaucoup (05).

Six de ces dix familles collaborent avec l'école. De plus, une mère déclare qu'elle collabore uniquement avec la nouvelle école, où elle sent que son fils a été bien accueilli. Par ailleurs, trois parents des classes moyenne et moyenne-supérieure affirment ne pas avoir le temps de faire du bénévolat à l'école en raison de leur travail et deux autres critiquent cette manière de fonctionner. Ces deux derniers parents entretiennent une relation conflictuelle avec l'école: C'est toujours de faire du bénévolat avec eux (52).

La principale activité de bénévolat des parents à l'école consiste à accompagner le groupe d'élèves dont fait partie leur enfant, lors des sorties scolaires: Je vais parfois aux sorties même si je dois faire garder le bébé. Je veux montrer à ma fille que je suis là pour elle (107).

Les autres activités consistent à aider le personnel scolaire lors des repas communautaires: Je suis allée préparer les pommes de terre, les servir (20).

De plus, les parents s'impliquent en effectuant de la surveillance aux heures de dîner et en prenant en charge des tâches inhérentes à la préparation et la réalisation d'activités culturelles et sportives: J'ai pas le temps de faire plus, sauf les olympiades une fois par année (106).

\subsubsection{L'appartenance à un comité de parents}

Un autre type d'implication parentale dans la vie scolaire de l'enfant consiste à faire partie d'un comité de parents. Toutefois, aucun parent vivant en contexte de pauvreté ne fait partie du comité d'école, bien qu'au moins un déclare vouloir le faire éventuellement: Je voudrais faire partie $d u$ comité d'école (45).

Également, parmi les six parents de la classe moyenne, une seule mère fait partie du conseil d'administration de la garderie scolaire alors qu'elle est en conflit avec l'école. Elle justifie sa participation en se déclarant satisfaite des services que son fils y reçoit. À l'inverse, une autre mère qui éprouve des tensions avec l'école n'est pas favorable à l'idée de participer à de tels comités: Disons que je ne suis pas portée à m'impliquer dans des comités à cette école-là en particulier (111). 
Par ailleurs, trois parents de la classe moyenne-supérieure mentionnent que le manque de temps les empêche de faire partie du comité d'école ou d'un autre comité de parents. À ceux qui mentionnent le manque de temps s'ajoutent les deux autres parents qui se font remplacer par leur mère, pour un total de huit parents justifiant le fait qu'ils ne s'impliquent pas pour cette même raison: J'ai essayé de faire partie de l'association de parents, mais je n'ai pas le temps (01).

\subsubsection{L'aide aux devoirs et aux leçons à la maison}

Le sixième des parents de l'échantillon s'impliquent dans l'aide aux devoirs et aux leçons à la maison. Sur ce nombre, un sur trois vit en contexte de pauvreté tandis qu'un parent sur sept fait partie de la classe moyenne ou moyenne-supérieure.

L'aide à la maison peut également consister à prendre connaissance des tâches que l'enfant doit réaliser ou à apporter son soutien en relation avec les tâches scolaires connexes. À titre d'exemple, une mère fait du rattrapage avec son fils, en révisant avec lui des concepts abordés au cours de l'année scolaire précédente.

\subsubsection{La participation à un groupe de parentage}

La participation à un groupe de parentage est une forme d'implication parentale dans la vie scolaire qui bénéficie indirectement à l'enfant. À cet effet, le tiers des parents de l'échantillon participent à de tels groupes, en faisant suite ou non à l'invitation de l'école: L'école m'envoie à des cours pour les comportements (88).

Trois parents vivant en contexte de pauvreté indiquent que l'école a fait pression sur eux pour qu'ils participent à un groupe de parentage. Ces pressions visent à ce que les pratiques éducatives parentales soient davantage efficaces à la maison tout en restant conformes à celles de l'école. Cette demande établit une frontière claire entre le rôle de l'école et celui des parents (Christenson et Sheridan, 2001). Par ailleurs, la conception de l'implication parentale dans la vie scolaire de l'enfant semble davantage hiérarchique dans le cas des parents vivant dans un contexte de pauvreté (Claes et Comeau, 1996; Comeau et Salomon, 1994): Ils veulent que je prenne plus de temps avec lui à la maison, mais pas en classe ni dans la cour d'école (97). 
Dans de très rares cas, soit lorsque l'école ne dispose pas de ressources suffisantes, le parent peut être invité à venir soutenir son enfant directement en classe: L'école m'a demandé de venir enseigner à mon enfant lorsqu'elle avait de la difficulté (46). Les types d'implication parentale dans la vie scolaire de l'enfant selon le milieu socioéconomique sont présentés au tableau 4.1.

\section{Tableau 4.1}

\section{Les types d'implication parentale dans la vie scolaire de l'enfant selon le milieu socioéconomique $(n=60)$}

\begin{tabular}{|c|c|c|c|c|}
\hline \multirow[b]{3}{*}{$\begin{array}{l}\text { Parents vivant en contexte } \\
\text { de pauvreté }\end{array}$} & \multicolumn{2}{|c|}{ Mères } & \multicolumn{2}{|c|}{ Pères } \\
\hline & $N$ & $\%$ & $N$ & $\%$ \\
\hline & 22 & 96,0 & 1 & 4,0 \\
\hline S'impliquent & 16 & 69,5 & 1 & 4,0 \\
\hline $\begin{array}{l}\text { Participent à un groupe } \\
\text { de parentage }\end{array}$ & 7 & 30,5 & & \\
\hline $\begin{array}{l}\text { Aident aux devoirs } \\
\text { et aux leçons à la maison }\end{array}$ & 6 & 26,0 & 1 & 4,0 \\
\hline $\begin{array}{l}\text { Participent à des activités } \\
\text { de bénévolat }\end{array}$ & 3 & 13,0 & & \\
\hline \multicolumn{5}{|l|}{ Font partie d'un comité de parents } \\
\hline & \multicolumn{2}{|c|}{ Mères } & \multicolumn{2}{|c|}{ Pères } \\
\hline & $N$ & $\%$ & $N$ & $\%$ \\
\hline $\begin{array}{l}\text { Parents des classes moyenne } \\
\text { et supérieure }\end{array}$ & 32 & 86,5 & 5 & 13,5 \\
\hline S'impliquent & 23 & 62,0 & 3 & 8,0 \\
\hline $\begin{array}{l}\text { Participent à des activités } \\
\text { de bénévolat }\end{array}$ & 10 & 27,0 & 1 & 3,0 \\
\hline Font partie d'un comité de parents & 5 & 13,5 & 1 & 3,0 \\
\hline $\begin{array}{l}\text { Aident aux devoirs } \\
\text { et aux leçons à la maison }\end{array}$ & 4 & 11,0 & 1 & 3,0 \\
\hline $\begin{array}{l}\text { Participent à un groupe } \\
\text { de parentage }\end{array}$ & 4 & 11,0 & & \\
\hline
\end{tabular}

\subsection{L'IMPLICATION PARENTALE SELON TROIS VARIABLES SOCIODÉMOGRAPHIQUES}

Pour près des trois quarts des parents de l'échantillon, il demeure capital de s'impliquer dans la vie scolaire de leur enfant. Des distinctions sont toutefois observables selon trois variables sociodémographiques, telles 
que l'âge de l'enfant, le niveau de scolarité des parents et le milieu socioéconomique des familles. L'analyse des résultats selon ces variables permet de mieux comprendre certains enjeux relatifs à la collaboration entre les milieux scolaire et familial en regard de la problématique des comportements violents au préscolaire et au primaire.

\subsubsection{L'âge de l'enfant}

L'âge de l'enfant semble être un facteur déterminant puisque les parents ont tendance à s'impliquer davantage dans la vie scolaire lorsque celui-ci est au début de son processus de scolarisation et de socialisation (Deslandes, 2001; Eccles et Harold, 1993; Epstein, 1995; Montandon, 1996; OCDE, 1997).

Les données de la présente recherche se situent dans le sens des écrits puisqu'au préscolaire, les trois quarts des parents qui s'impliquent ont un enfant de 3 à 4 ans, alors que plus de huit parents sur dix ont un enfant de 4 à 6 ans. Au primaire, sur le nombre de parents qui s'impliquent, plus de six parents sur dix ont un enfant de 6 à 8 ans et le tiers d'entre eux ont un enfant de 8 à 9 ans. L'implication parentale selon le nombre d'enfants par groupe d'âge est présentée au tableau 4.2.

TABleau 4.2

L'implication parentale selon le nombre d'enfants par groupe d'âge $(n=43)$

\begin{tabular}{lcrccc}
\hline \multirow{2}{*}{$\begin{array}{l}\text { Nombre } \\
\text { d'enfants }\end{array}$} & Âge & $\boldsymbol{N}$ & $\%$ & $\boldsymbol{N}$ & $\%$ \\
\hline & & 39 & 91,5 & 4 & 8,5 \\
\hline 4 & 3 à 4 ans & 3 & 75,0 & & \\
\hline 7 & 4 à 5 ans & 6 & 86,0 & & \\
\hline 12 & 5 à 6 ans & 10 & 83,5 & & \\
\hline 12 & 6 à 7 ans & 8 & 66,5 & 2 & 16,5 \\
\hline 11 & 7 à 8 ans & 7 & 63,5 & 2 & 16,5 \\
\hline 14 & 8 à 9 ans & 5 & 36,0 & & \\
\hline
\end{tabular}




\subsubsection{Le niveau de scolarité des parents}

Le niveau de scolarité des parents joue un rôle très important puisque plus il est élevé, plus ils ont tendance à s'impliquer dans la vie scolaire de leur enfant. À ce sujet, les parents qui détiennent une formation de niveau universitaire sont davantage conscients de l'importance de soutenir leur enfant dans son processus de scolarisation et de socialisation que les moins scolarisés (Pourtois et Desmet, 1997).

Les parents les plus scolarisés affichent davantage d'esprit critique et font davantage preuve que les autres d'une attitude de consommateurs de services à l'égard du milieu scolaire (de Singly, 2000; Dubet, 1997; Dubet et Martuccelli, 1996; Hirschhon, 2001; Pourtois et Desmet, 1997; Vincent et Tomlinson, 1997; Van Zanten et Duru-Bellat, 1999). Le consumérisme les ouvre à une implication active ainsi qu'à un empowerment accru (Conseil de la famille et de l'enfance, 2000) qui, par ailleurs, peut s'avérer une source de conflits avec l'école. Une telle attitude peut se traduire par des tensions, voire par une relation conflictuelle entre l'école et les parents, en raison d'échanges qui correspondent moins à de la collaboration qu'à de la méfiance et à de l'opposition entre eux (Perrenoud, 2001a, 2001b).

On observe aussi un fossé entre l'école et certains parents moins scolarisés. À ce sujet, les trois quarts des parents qui vivent en contexte de pauvreté, soit majoritairement des jeunes mères de familles monoparentales, détiennent au plus un diplôme d'études secondaires. Ces mères se sentent souvent incompétentes ou peu compétentes pour aider leur enfant dans le processus de scolarisation et de socialisation (Epstein, 1992). Face à ce sentiment, elles ont tendance à transférer la compétence aux enseignants, qui peuvent les percevoir selon des cadres de référence hiérarchiques (Kohl, Lengua et McMahon, 2000; Marcon, 1999). Le fossé entre l'école et ces mères peut générer de grandes frustrations chez elles: Il se fait bûcher dessus à l'école à cause de ce système de points. Ça n'a pas de bon sens. Il en a ben d'autres systèmes qui ne vont pas tous les dévaloriser, leur taper sur la personnalité et les discriminer comme ça (11.1); Des fois, l'école ne respecte pas le parent et sa manière de faire avec son enfant. Ils nous impliquent seulement parce que la loi l'oblige (112).

Les données de la présente recherche se situent dans le sens des écrits puisque la moitié des parents qui s'impliquent dans la vie scolaire de leur enfant détiennent un diplôme d'études élémentaires, tandis qu'un peu plus de la moitié détiennent un diplôme d'études secondaires. Par ailleurs, les trois quarts de ceux qui s'impliquent ont un diplôme d'études collégiales ou universitaire. L'implication parentale selon le nombre de parents par niveau de scolarité est présentée au tableau 4.3. 
TABLEAU 4.3

L'implication parentale selon le nombre de parents par niveau de scolarité $(n=43)$

\begin{tabular}{llrcrc}
\hline \multirow{2}{*}{$\begin{array}{l}\text { Nombre } \\
\text { d'enfants }\end{array}$} & Niveau & \multicolumn{2}{c}{ Mères } & \multicolumn{3}{c}{ Pères } \\
\cline { 3 - 6 } & de scolarité & $N$ & $\%$ & $N$ & $\%$ \\
\hline 2 & Élémentaire & 39 & 91,5 & 4 & 8,5 \\
\hline 24 & Secondaire & 13 & 54,0 & 1 & 4,0 \\
\hline 16 & Collégial & 12 & 75,0 & 1 & 6,0 \\
\hline 17 & Universitaire & 13 & 76,5 & 2 & 12,0 \\
\hline $1^{*}$ & $\mathrm{n} / \mathrm{d}$ & 0 & 0,0 & 0 & 0,0 \\
\hline
\end{tabular}

* Le parent dont le niveau de scolarité n'est pas disponible ne s'implique pas dans la vie scolaire de son enfant.

\subsubsection{Le milieu socioéconomique des familles}

Si le niveau de scolarité des parents constitue un important facteur prédicteur de l'implication parentale dans la vie scolaire de leur enfant (Dubet et Martuccelli, 1996 ; Epstein, 1995 ; Grolnick, Benjet, Kurowski et Apostoleris, 1997), le milieu socioéconomique des familles peut parfois le surpasser (Kohl, Lengua et McMahon, 2000).

Les données de la présente recherche se situent en partie dans le sens des écrits puisque les parents de la classe supérieure s'impliquent légèrement en plus grand nombre dans la vie scolaire de leur enfant que ceux des classes pauvre, moyenne et moyenne-supérieure. Par ailleurs, tous les parents de l'échantillon vivant sous le seuil de la pauvreté s'impliquent dans la vie scolaire de leur enfant. L'hypothèse pouvant expliquer cette situation est que ces parents n'occupent pas d'emploi à temps plein et qu'ils ont, par conséquent, davantage de temps disponible pour s'impliquer. L'implication parentale selon le nombre de familles par milieu socioéconomique est présentée au tableau 4.4. 
TABLEAU 4.4

L'implication parentale selon le nombre de familles par milieu socioéconomique $(n=43)$

\begin{tabular}{llcccc}
\hline \multirow{2}{*}{$\begin{array}{l}\text { Nombre } \\
\text { d'enfants }\end{array}$} & $\begin{array}{l}\text { Milieu } \\
\text { socioéconomique }\end{array}$ & $\boldsymbol{N}$ & $\%$ & $\boldsymbol{N}$ & $\%$ \\
\cline { 3 - 6 } & & 39 & 91,5 & 4 & 8,5 \\
\hline 11 & $\begin{array}{l}\text { Sous le seuil } \\
\text { de la pauvreté }\end{array}$ & 10 & 91,0 & 1 & 9,0 \\
\hline 12 & Classe pauvre & 6 & 50,0 & & \\
\hline 12 & Classe moyenne & 7 & 58,5 & 1 & 8,5 \\
\hline 12 & $\begin{array}{l}\text { Classe } \\
\text { moyenne-supérieure }\end{array}$ & 7 & 58,5 & 1 & 8,5 \\
\hline 13 & Classe supérieure & 9 & 69,0 & 1 & 7,5 \\
\hline
\end{tabular}

\subsubsection{En résumé}

Plus des trois quarts des parents de l'échantillon considèrent que l'école les invite à s'impliquer dans quatre des six types d'activités décrits dans la typologie d'Epstein (1995), soit le bénévolat à l'école, l'appartenance à un comité de parents, l'aide aux devoirs et aux leçons à la maison et la participation à un groupe de parentage.

Lorsqu'on compare le type d'implication parentale dans la vie scolaire selon le milieu socioéconomique des familles, on observe des distinctions notables. En effet, les parents regroupés dans les classes moyenne et moyenne-supérieure s'impliquent davantage dans les activités de bénévolat à l'école et dans des comités de parents que ceux vivant en contexte de pauvreté, qui préfèrent le faire à la maison. La sous-représentation de ces parents dans ces activités va dans le sens des principaux écrits sur le sujet, à savoir que leur absence d'implication à l'école est compensée par leurs propres pratiques ou formes d'engagement auprès de leur enfant à la maison (Dubet et Martuccelli, 1996; Epstein, 1995; Grolnick, Benjet, Kurowski et Apostoleris, 1997; Lareau, 1987; Lytton et Pyryt, 1998; Marcon, 1999 ; Pelco, Jacobson, Ries et Melka, 2000; Raffaele et Knoff, 1999): Si ça a affaire avec mon petit, je vais faire tout ce que je peux, mais si ça a affaire avec l'école, c'est non! S'ils me demandent comme parent pour aller au musée, c'est non! Du «school outing», c'est non! (92).

Quelques éléments militent en faveur de cette prise de position par les familles de l'échantillon vivant en contexte de pauvreté, à savoir que plus des trois quarts d'entre elles sont monoparentales. Selon Grolnick, Benjet, Kurowski et Apostoleris (1997), les mères qui sont chefs d'une famille monoparentale s'impliquent moins à l'école que les parents de familles 
intactes. De plus, Kohl, Lengua et McMahon (2000) maintiennent qu'en raison d'un manque de temps, elles accordent la priorité aux contacts avec l'enseignant plutôt qu'à des activités de bénévolat. Dans le prolongement de ces constats, les familles vivant en contexte de pauvreté de l'échantillon s'impliquent davantage dans l'aide aux devoirs et aux leçons à la maison ainsi que dans des groupes de parentage que ne le font celles des classes moyenne et moyenne-supérieure.

Aux familles vivant en contexte de pauvreté s'ajoutent celles qui vivent en contexte minoritaire pluriethnique. Celles-ci ne maîtrisent bien ni le français, ni l'anglais étant nouvellement arrivées au Canada. Selon McAndrew (2001), l'ethnicité coïncide souvent avec les difficultés socioéconomiques. Toutefois, l'origine ethnoculturelle des parents et la langue parlée à la maison se révèlent des facteurs limitant davantage l'implication parentale à l'école que le milieu socioéconomique (Desimone, 1999; Eccles et Harold, 1993 ; Kohl, Lengua et McMahon, 2000 ; Lareau et Horvat, 1999; Lee Yao, 1993; Rich, 1993). De telles limites chez ces parents font qu'ils se sentent souvent intimidés par le personnel et la structure scolaires, qu'ils considèrent difficiles d'accès (Peña, 2000).

En marge de l'analyse des données par variables sociodémographiques, telles que l'âge des enfants, le niveau de scolarité des parents, le statut, l'origine ethnoculturelle, la langue ou le milieu socioéconomique des familles, l'implication parentale dans la vie scolaire de l'enfant repose sur une communication positive entre les milieux scolaire et familial.

\subsection{LA COMMUNICATION ENTRE LES MILIEUX SCOLAIRE ET FAMILIAL}

La communication s'avère le fondement de la collaboration entre l'école et les parents. Cette communication est d'autant plus importante dans le cas d'enfants ayant des comportements violents au préscolaire et au primaire afin de contrer, voire surmonter, la situation-problème (Adams et Christenson, 2000 ; Christenson et Sheridan, 2001). La distinction établie entre les parents selon le milieu socioéconomique par rapport à l'implication parentale dans la vie scolaire de l'enfant se vérifie également dans la communication entre l'école et les parents.

Le tiers des parents de l'échantillon, soit un peu plus du quart de ceux qui vivent en contexte de pauvreté et presque la moitié de ceux des classes moyenne et moyenne-supérieure, déclarent que la communication avec l'école est bonne: On se parle et on s'écoute (12); La communication entre parent et enseignant, parent et directeur, c'est très bon (96); On a une très bonne communication (102). 
D'après ces parents, le personnel scolaire est disponible pour les aider, ainsi que clarifier et donner de l'information. De plus, ils perçoivent une ouverture et se sentent soutenus par l'école: Quand ils voient un problème, ils s'impliquent pour être certains que le parent soit tenu au courant (24).

Lorsque la communication n'est pas bonne entre l'école et les parents, des tensions s'installent et provoquent de fortes réactions chez ces derniers: L'école dicte aux parents comment agir. On peut pas faire grandchose, nous autres. On est juste des numéros (06); C'est moi qui dois leur courir après comme une malade (92). Une fois la relation tendue, il devient difficile de rétablir la bonne entente et la communication positive entre les deux milieux, à moins d'un travail effectué en ce sens: À la première école, la façon qu'il m' «achalait» [il m'importunait], c'est pas juste. Il me «pageait» [il m'envoyait des messages sur mon téléavertisseur] presqu'à tous les jours, puis j'étais en classe. Je les rappelais tout de suite, c'était du trouble horrible. Puis, je l'ai changé d'école (112).

\subsubsection{La fréquence des contacts au sujet de la situation-problème de l'enfant}

Les trois quarts des parents de l'échantillon mentionnent avoir des contacts fréquents avec le milieu scolaire au sujet de la situation-problème de leur enfant. Toutefois, plus de quatre parents sur cinq des classes moyenne et moyenne-supérieure, comparativement à deux parents sur trois vivant en contexte de pauvreté, considèrent avoir de tels contacts avec le milieu scolaire concernant leur enfant.

Ces contacts fréquents entre l'école et les parents, s'ils reposent sur une communication positive, contribuent à un rapprochement entre les deux milieux. Notamment, ils permettent de mieux connaître l'enfant, de mieux cerner ses difficultés comportementales et, par conséquent, de mieux intervenir lorsqu'elles surviennent: J'ai pris un rendez-vous avec la direction pour parler de moi-même et de mon enfant (74); On ne se cache rien (107).

\subsubsection{La disponibilité et l'ouverture à l'endroit du milieu scolaire}

Pour près du tiers des parents de l'échantillon, la disponibilité et l'ouverture à l'endroit du milieu scolaire sont les éléments essentiels d'une communication positive. À ce sujet, les parents mentionnent que ces éléments constituent les fondements sur lesquels repose leur manière de communiquer avec l'école: J'essaie d'être aussi complémentaire que je peux avec eux (01); Je suis toujours prête à leur parler (09); Je n'ai rien caché. J'ai été honnête avec eux (10); Je leur parle ouvertement (15); Je leur ai dit de m'appeler n'importe quand (110); J'ai même acheté une "pagette» [téléavertisseur] (112). 
À l'inverse, face à la disponibilité et à l'ouverture dont l'école leur témoigne, les parents se sentent plus désireux de collaborer et entretiennent une perception davantage positive à l'endroit du personnel scolaire.

\subsubsection{Les moyens de communication}

Un peu plus du tiers des parents de l'échantillon précisent avoir assisté à des réunions spéciales concernant la situation-problème de leur enfant: Il y a eu des rencontres: les rencontres ordinaires, celles du bulletin et aussi celles pour son plan individuel (20).

En ce qui a trait aux moyens de communication écrits, un parent sur deux déclare privilégier les lettres et encore davantage l'agenda scolaire. Sur ce dernier moyen, la distinction selon le milieu socioéconomique des parents se confirme à savoir que les trois cinquièmes de ceux qui sont regroupés dans les classes moyenne et moyenne-supérieure en mentionnent l'usage régulier, comparativement au cinquième seulement de ceux qui vivent en contexte de pauvreté: C'est moi qui ai demandé pour faire des notes dans l'agenda scolaire à chaque jour parce que des fois, quelque chose arrivait mardi, puis je le savais juste jeudi. J'ai demandé, s'il y avait des problèmes, de l'écrire tout de suite (96); Alors, avec l'agenda scolaire, au moins je peux savoir si ça va bien ou pas (109).

Par ailleurs, les appels téléphoniques à l'école constituent le moyen de communication privilégié par le quart des parents de l'échantillon. Le tiers de ceux qui en font un usage régulier vivent en contexte de pauvreté: La communication est régulière, par téléphone ou par des visites à l'école (10); Lorsqu'il y a eu une situation, le professeur téléphonait pour m'informer qu'elle s'occuperait de mon fils (83).

Également, le tiers des parents de l'échantillon n'hésitent pas à faire une visite à l'école en vue d'obtenir de l'information auprès du personnel scolaire. Il s'agit également d'un moyen de communication davantage privilégié par les parents vivant en contexte de pauvreté: Je $m^{\prime} y$ rends souvent (15); J'ai parlé à la surveillante de l'heure du dîner parce que c'est la gardienne de mon petit le matin (12.1); Je m'informe auprès de l'enseignante comment ça se passe avec mon fils, puisque je fais de la surveillance sur l'heure du dîner (23); Quand j'ai la chance, je vais voir le professeur (102).

Finalement, dans un dernier cas, la garderie scolaire est mise à contribution pour entretenir des échanges verbaux au quotidien: $\grave{A}$ tous les jours lorsque je viens chercher mon petit, si ça va bien, ils me le disent. Si ça va pas bien, ils me disent pourquoi (97). Les dimensions d'une bonne communication entre l'école et les parents selon le milieu socioéconomique sont présentés au tableau 4.5 . 
TABLEAu 4.5

Les dimensions d'une bonne communication entre l'école et les parents selon le milieu socioéconomique $(n=60)$

\begin{tabular}{|c|c|c|c|c|}
\hline \multirow[b]{3}{*}{$\begin{array}{l}\text { Parents vivant en contexte } \\
\text { de pauvreté }\end{array}$} & \multicolumn{2}{|c|}{ Mères } & \multicolumn{2}{|c|}{ Pères } \\
\hline & $N$ & $\%$ & $N$ & $\%$ \\
\hline & 22 & 96,0 & 1 & 4,0 \\
\hline Ont des contacts fréquents & 15 & 65,0 & & \\
\hline Assistent à des réunions spéciales & 10 & 43,5 & & \\
\hline Effectuent des appels téléphoniques & 8 & 35,0 & & \\
\hline Font des visites à l'école & 7 & 30,5 & 1 & 4,0 \\
\hline Ont une bonne communication & 6 & 26,0 & 1 & 4,0 \\
\hline $\begin{array}{l}\text { Sont disponibles et ouverts } \\
\text { face à l'école }\end{array}$ & 6 & 26,0 & 1 & 4,0 \\
\hline $\begin{array}{l}\text { Font un usage régulier } \\
\text { de l'agenda scolaire }\end{array}$ & 5 & 22,0 & & \\
\hline \multicolumn{5}{|l|}{$\begin{array}{l}\text { Entretiennent des échanges } \\
\text { verbaux quotidiens }\end{array}$} \\
\hline & \multicolumn{2}{|c|}{ Mères } & \multicolumn{2}{|c|}{ Pères } \\
\hline & $N$ & $\%$ & $N$ & $\%$ \\
\hline $\begin{array}{l}\text { Parents des classes moyenne et } \\
\text { moyenne supérieure }\end{array}$ & 32 & 86,5 & 5 & 13,5 \\
\hline Ont des contacts fréquents & 29 & 78,0 & 1 & 3,0 \\
\hline $\begin{array}{l}\text { Font un usage régulier de } \\
\text { l'agenda scolaire }\end{array}$ & 26 & 70,0 & 1 & 3,0 \\
\hline Ont une bonne communication & 17 & 46,0 & 1 & 3,0 \\
\hline $\begin{array}{l}\text { Sont disponibles et ouverts } \\
\text { face à l'école }\end{array}$ & 11 & 30,0 & 1 & 3,0 \\
\hline Réunions spéciales & 11 & 30,0 & 1 & 3,0 \\
\hline Font des visites à l'école & 7 & 19,0 & 1 & 3,0 \\
\hline $\begin{array}{l}\text { Effectuent des appels } \\
\text { téléphoniques }\end{array}$ & 6 & 16,0 & 1 & 3,0 \\
\hline $\begin{array}{l}\text { Entretient des échanges } \\
\text { verbaux quotidiens }\end{array}$ & 1 & 3,0 & & \\
\hline
\end{tabular}




\subsubsection{En résumé}

Les moyens de communication directs, tels que la visite à l'école ou l'appel téléphonique, sont davantage privilégiés par les parents vivant en contexte de pauvreté que les moyens indirects ou écrits, tels que les rendez-vous officiels, les lettres et même l'agenda scolaire.

À ce sujet, une mère vivant en contexte de pauvreté dit laisser un message sur la boîte vocale de l'enseignante à tous les jours, alors qu'une autre apprécie recevoir un appel de la secrétaire lorsque son fils quitte l'école: Je l'appelle à tous les matins (85); C'est eux autres qui me téléphonent. Même quand il part de l'école, ils vont m'appeler pour me le dire: "Il vient juste de partir» (94).

\subsection{LES DIFFICULTÉS DE COMMUNICATION}

Selon Christenson et Sheridan (2001), la communication servant d'assise à la collaboration entre l'école et les parents est bidirectionnelle, enrichissante et productive. De plus, elle repose sur une confiance et un soutien mutuels.

Malgré les efforts déployés par certains parents pour y parvenir, de même que les nombreux contacts qu'ils entretiennent avec l'école, il subsiste des difficultés de communication entre les milieux scolaire et familial. Si la fréquence de ces contacts est généralement associée à une bonne communication, elle peut également aller de pair avec les difficultés encourues par l'enfant (Kohl, Lengua et McMahon, 2000; Marcon, 1999; Reynolds, Weissberg et Kasprow, 1992). En ce sens, la teneur des propos échangés au cours de ces contacts peut facilement être génératrice de tensions entre les deux milieux. C'est pourquoi la qualité de la communication et le contenu positif des messages l'emportent sur la quantité des échanges dans l'instauration d'une bonne collaboration entre l'école et les parents (Adams et Christenson, 2000; Reynolds, Weissberg et Kasprow, 1992).

Également, selon certains parents, au manque de communication peut parfois s'ajouter un manque d'écoute et d'ouverture de la part du personnel scolaire.

\subsubsection{Le manque de communication}

Plus du tiers des parents de l'échantillon perçoivent un manque parfois important de communication avec l'école. Dans les mêmes proportions, d'autres expriment un malaise quant au type d'informations échangées. À ce sujet, ces parents déclarent qu'ils ne se sentent pas informés sur des 
aspects importants concernant leur enfant. Par rapport à cette situation, le quart des parents éprouvent un sentiment d'absence de collaboration de la part du personnel scolaire, pouvant parfois mener à un conflit.

Selon un parent sur dix, l'école ne retourne pas les appels téléphoniques ou n'accuse pas réception des lettres qu'on leur envoie: On avait beau demander des choses à l'avance, il n'y avait aucune réponse (01); Finalement, moi, personne ne m'a jamais rappelée (11.1); Je n'ai eu aucune nouvelle de l'école suite à cette démarche (12.1); Quand j'essayais d'appeler le conseil scolaire, il ne retournait pas mes appels (84); Puis, moi, je vais écrire quelque chose après. Ça fait "une couple de fois» [quelques fois] que j'ai rien. J'ai pas de retour (90).

Cette perception peut parfois s'élargir et englober d'autres intervenants associés au milieu scolaire: J'ai tenté de rejoindre le comité de parents, mais on ne m'a jamais rappelée. J'ai laissé des messages à des psychologues qui ne m'ont pas rappelée. Je ne sais pas, j'ai l'impression que j'ai un virus de «Rappelez-moi pas!» (11.1).

Également, certains parents estiment trop long le délai avant qu'on les informe d'un événement fâcheux: La direction a attendu un mois avant de dire qu'il y avait un problème avec mon fils (38); J'ai aucune idée de qu'est-ce qui est arrivé. Ça fait deux jours, puis je le sais toujours pas (92).

D'autres parents se plaignent d'une absence de régularité dans les communications: C'est des choses que j'ai demandé de savoir depuis l'année passée (05); Tellement de parents, puis tellement d'enfants en même temps. Il n'y a presque pas de communication entre la garderie et les parents. Ça devrait être plus organisé. On devrait communiquer avec les parents une fois par mois, pour un bon "update » sur les enfants (11); Qu'il ne suivait pas les consignes, pourquoi je ne l'ai pas su dès le début? Pour la violence, ce fut sur-le-champ! (114).

Quelques autres parents se plaignent d'un filtrage dans la transmission de certaines informations. Il est à noter que cette dernière famille a été l'objet d'un signalement à la Société de l'aide à l'enfance (SAE) et qu'une telle intervention exige de ne pas en informer le parent: Je fais juste lui dire constamment que je veux savoir ce qui se passe. Puis, lui, il pense que je ne devrais pas le savoir, sauf que je veux le savoir, moi! (05); Je suis frustrée parce que la direction ne m'a pas dit que mon enfant allait voir la travailleuse sociale de l'école (12.1); Des fois, ils enlèvent des privilèges à l'école et je n'en suis pas informée (83).

Quelquefois, après différents échanges avec le personnel scolaire, des parents espèrent recevoir une confirmation de ce qui a été dit ou des engagements qui ont été pris. Toutefois, il en résulte souvent de la déception face à cette attente non comblée par l'école: Ils ne m'ont rien confirmé 
au début de l'année. Ils ne m’ont jamais confirmé qu'il a recommencé à voir la psychologue (05); Mon enfant, il faut qu'il prenne une pompe. Mais l'école oublie toujours de la lui donner (102).

Également, quelques parents considèrent que l'école ne transmet pas suffisamment d'informations positives. En ce sens, ils ont l'impression que l'école communique essentiellement avec eux pour leur transmettre des informations négatives: Et puis, dans l'agenda scolaire, j'ai commencé à voir qu'à tous les jours, il y avait quelque chose de mauvais. Jamais, jamais, jamais c'était positif. C'était tout le temps négatif (109).

Cette perception va dans le sens des écrits, à savoir que l'école a tendance à ne rejoindre les parents que lorsque l'enfant éprouve des difficultés (Deslandes, 2001; Marcon, 1999). Lorsque son comportement s'améliore, l'école ne prend pas la peine de le communiquer aux parents: C'est à cause que j'avais tellement de conversations avec la direction quand ça allait mal. J'ai pas eu un seul appel depuis que ça va bien (111).

\subsubsection{Le manque d'écoute et d'ouverture du personnel scolaire}

Selon l'ensemble des parents de l'échantillon, la qualité de la communication avec l'école est très importante. Toutefois, le tiers d'entre eux croient que le personnel scolaire fait parfois preuve d'un manque d'écoute.

Pour quatre parents sur cinq, il est plus difficile d'entretenir une bonne collaboration puisque ce manque d'écoute s'accompagne parfois d'un manque d'ouverture. L'ouverture est définie comme étant la capacité $\mathrm{du}$ personnel scolaire de recevoir des commentaires et des propositions de la part des parents pouvant enclencher un processus de changement tout en remettant en question leur pratique professionnelle établie (Gohier, 1996): J'ai pu discuter seulement une ou deux fois avec l'enseignante parce que j'étais pas d'accord avec son attitude et ses méthodes. Mais par après, elle a refusé d'entendre quoi que ce soit (48).

Des parents notent aussi des difficultés dans les échanges avec la direction d'école: La directrice, c'était quelqu'un qui disait quelque chose, puis qui faisait le contraire. On lui disait quelque chose, ça lui rentrait par une oreille, puis ça lui sortait par l'autre (106).

Une communication déficiente accompagnée d'un manque d'écoute et d'ouverture peut facilement entraîner une confusion dans les perceptions, qui engendre et nourrit les tensions: Je voulais parler à l'enseignante, qui ne voulait rien savoir de moi. Et l'enseignante disait qu'elle ne m'avait pas renvoyée comme ça. Elle a dit: "J'ai cru que vous étiez pressée, que vous vouliez rentrer chez vous, que vous ne vouliez pas me parler de la situation!»(01). 
Certains parents se disent parfois insatisfaits face à la rigidité des positions prises par les enseignants au sujet de leurs pratiques pédagogiques, jugeant qu'elles ne répondent pas toujours adéquatement à leurs demandes et à leurs préoccupations: Le seul feedback que j'ai eu, c'est mon fils qui, en revenant de l'école m'a dit: Maman, la maîtresse $m^{\prime} a$ dit de te dire qu'elle va encore avoir des numéros parce qu'elle ne veut pas changer son système pour personne (11.1); La maîtresse m'a répondu que ma fille était pas assez faible pour une classe d'appui. Point final! (106).

Finalement, dans un cas, la direction d'école a ignoré les propositions d'une mère afin de faciliter leur rencontre: Pour moi, c'est très difficile d'avoir des rencontres le jour. Je suggérais le soir. Mais c'était jamais possible. Donc, je lui ai dit: "Bon, bien, si vous voulez toujours me rencontrer, je vais être à la rencontre de parents. Pourquoi ne pas être là vous autres aussi!» (05). Les dimensions d'une communication difficile entre l'école et les parents sont présentés au tableau 4.6.

\subsubsection{En résumé}

Les trois quarts des parents établissent des contacts fréquents avec le personnel scolaire, relativement à la situation-problème de leur enfant à l'école, soit plus de quatre parents sur cinq des classes moyenne et moyenne-supérieure et de deux sur trois vivant en contexte de pauvreté. Toutefois, la teneur des propos échangés lors de ces contacts peut facilement générer des tensions, car la fréquence des échanges est souvent associée aux difficultés encourues par l'enfant (Kohl, Lengua et McMahon, 2000; Marcon, 1999; Reynolds, Weissberg et Kasprow, 1992). Ainsi, la qualité de l'échange et le contenu positif des messages l'emportent sur la quantité de contacts en vue d'établir une bonne collaboration entre l'école et les parents (Adams et Christenson, 2000; Reynolds, Weissberg et Kasprow, 1992).

En plus d'un manque de communication, certains parents perçoivent un manque d'écoute et d'ouverture de la part du personnel scolaire à l'égard de leurs commentaires et de leurs propositions. Ces manques peuvent $s^{\prime}$ inscrire dans la délimitation de frontières claires avec le milieu familial. Paradoxalement, Christenson et Sheridan (2001) avancent que le maintien de telles frontières est la caractéristique d'une bonne collaboration. $\mathrm{Si}$, dans certains cas, elles peuvent s'avérer positives, ces frontières peuvent également consolider les difficultés de communication existantes entre l'école et les parents et représenter une source de conflits entre eux. Selon certains parents, les difficultés de communication sont souvent propices à l'émergence d'un conflit avec l'école. 
TABleAu 4.6

Les dimensions d'une communication difficile entre l'école et les parents $(n=60)$

\begin{tabular}{|c|c|c|c|c|}
\hline & \multicolumn{2}{|c|}{ Mères } & \multicolumn{2}{|c|}{ Pères } \\
\hline & $N$ & $\%$ & $N$ & $\%$ \\
\hline & 54 & 90,0 & 6 & 10,0 \\
\hline $\begin{array}{l}\text { Notent un manque de } \\
\text { communication }\end{array}$ & 20 & 33,5 & 2 & 3,5 \\
\hline $\begin{array}{l}\text { Ont du malaise sur le type de } \\
\text { renseignements échangés }\end{array}$ & 20 & 33,5 & 1 & 1,5 \\
\hline Se heurtent à un manque d'écoute & 19 & 31,5 & 1 & 1,5 \\
\hline $\begin{array}{l}\text { Se heurtent à un manque } \\
\text { d'ouverture }\end{array}$ & 16 & 26,5 & 1 & 1,5 \\
\hline $\begin{array}{l}\text { Éprouvent un sentiment d'absence } \\
\text { de collaboration }\end{array}$ & 15 & 25,0 & 1 & 1,5 \\
\hline $\begin{array}{l}\text { Ne reçoivent pas de retour } \\
\text { d'appels ou de lettres }\end{array}$ & 7 & 11,5 & 1 & 1,5 \\
\hline $\begin{array}{l}\text { Trouvent trop long le délai avant } \\
\text { qu'on les informe }\end{array}$ & 5 & 8,0 & 1 & 1,5 \\
\hline $\begin{array}{l}\text { Notent une absence de régularité } \\
\text { dans la communication }\end{array}$ & 5 & 8,0 & & \\
\hline $\begin{array}{l}\text { Constatent un filtrage de certaines } \\
\text { informations }\end{array}$ & 3 & 5,0 & 1 & 1,5 \\
\hline $\begin{array}{l}\text { Ne reçoivent pas de confirmation } \\
\text { après les échanges }\end{array}$ & 3 & 5,0 & & \\
\hline $\begin{array}{l}\text { Considèrent que les informations } \\
\text { ne sont pas positives }\end{array}$ & 3 & 5,0 & & \\
\hline $\begin{array}{l}\text { Notent des difficultés dans } \\
\text { les échanges }\end{array}$ & 2 & 3,5 & 1 & 1,5 \\
\hline $\begin{array}{l}\text { Sont insatisfaits face à la rigidité } \\
\text { des positions prises }\end{array}$ & 2 & 3,5 & & \\
\hline $\begin{array}{l}\text { N'apprécie pas que l'école ignore } \\
\text { les propositions }\end{array}$ & 1 & 1,5 & & \\
\hline
\end{tabular}

\subsection{UN COURT BILAN DES RÉSULTATS SUR LA COLLABORATION}

La collaboration entre l'école et les parents se définit comme un travail conjoint en vue d'atteindre des buts communs concernant l'enfant (Christenson et Sheridan, 2001). L'implication parentale dans la vie scolaire de l'enfant et dans les activités de bénévolat à l'école, représente le premier pas vers l'instauration d'une bonne collaboration entre les deux milieux. 
Celle-ci doit reposer sur une communication positive qui implique des échanges bidirectionnels, enrichissants et productifs et qui s'appuie ellemême sur une confiance et un soutien mutuels.

Or, selon leur niveau de scolarité et leur milieu socioéconomique, les parents ne privilégient pas les mêmes types d'implication dans la vie scolaire de leur enfant. Dans la foulée des écrits d'Epstein (1995), les parents des classes moyenne et moyenne-supérieure s'impliquent directement dans les activités à l'école. À l'inverse, les parents vivant en contexte de pauvreté, soit en grande majorité de jeunes mères de famille monoparentale, préfèrent s'impliquer auprès de leur enfant à la maison.

Cette distinction s'applique également aux échanges avec le personnel scolaire. Pour les moyens écrits et formels, tels que les lettres et l'agenda scolaire, la proportion de parents des classes moyenne et moyennesupérieure qui entretiennent de telles communications avec l'école est deux fois plus forte que celle des parents vivant en contexte de pauvreté. Ces derniers semblent davantage privilégier des moyens informels, tels qu'un appel téléphonique et une visite à l'école pour discuter de leur enfant. Ces parents ont leurs propres pratiques d'implication et de communication, voire de collaboration avec l'école.

En résumé, lorsque l'on considère toutes les distinctions qui s'appliquent entre les différentes situations des répondants, force est de constater que la collaboration entre l'école et les parents a davantage de chances de s'articuler au sujet de la situation-problème d'un enfant provenant des classes moyenne et moyenne-supérieure. Nombre d'auteurs ayant publié dans le domaine appuient ce constat (Desimone, 1999; Dubet et Martuccelli, 1996; Johnson, 1994; Nakagawa, 2000).

\section{LA PARTICIPATION DES PARENTS AU PROCESSUS DE PRISE DE DÉCISIONS}

Selon Christenson et Sheridan (2001), en plus d'une communication positive, une bonne collaboration entre l'école et les parents repose sur la prise de décisions communes concernant l'enfant. À ce sujet, trois questions de la grille d'entrevue parentale présentée à l'annexe 1 abordent spécifiquement la participation des parents au processus de prise de décisions concernant la situation-problème de leur enfant à l'école. Ces questions touchent à l'invitation adressée aux parents par l'école à participer à la recherche d'une solution (question 29), l'invitation à participer aux décisions à prendre concernant l'enfant (question 30) et l'influence des parents sur les décisions déjà prises par l'école (question 31). 
Bien que les parents aient tout d'abord répondu oui ou non à ces questions, ils avaient également la possibilité de donner plus de détails en formulant des commentaires propres à fournir un contexte à leur réponse. Par exemple, à la question 30, certains parents qui déclarent ne pas avoir participé aux décisions à prendre concernant leur enfant formulent dans leurs commentaires que c'est parce que l'école ne les a pas invités à le faire: C'étaient les règlements de l'école et du conseil. C'était obligatoire et une fois suspendu, il ne pouvait pas y retourner. Je n'avais rien à dire (82).

\subsection{L'INVITATION AUX PARENTS À PARTICIPER À LA RECHERCHE DE SOLUTIONS}

À la question 29, la moitié des parents de l'échantillon déclarent que l'école sollicite leur participation à la recherche d'une solution au sujet de la situation-problème de leur enfant. L'autre moitié perçoit, à l'inverse, que l'école n'invite pas les parents à participer à la recherche d'une telle solution: Elle nous a informés et puis, sans le dire, ils s'attendent à ce que les parents renforcent les messages de l'école (01).

Certains d'entre eux affirment même que l'école ne les a aucunement impliqués dans le processus décisionnel: Non. C'est pour ça que je n'ai pas aimé ça (15).

Par ailleurs, quelques parents des classes moyenne et moyennesupérieure répondent à la fois positivement et négativement à la question. Pour illustrer cette double perception, une mère dit que l'école ne lui accorde pas le moindre choix, qu'on lui demande des trucs mais qu'une fois suggérés, ils sont ignorés et même critiqués. Un dernier parent abonde dans le même sens lorsqu'il dit qu'en cas de désaccord, l'école ne tient pas compte de ses opinions. L'invitation aux parents à participer à la recherche de solutions est présentée au tableau 4.7. 
TABleau 4.7

L'invitation aux parents à participer à la recherche de solutions $(n=60)$

\begin{tabular}{lrrrr}
\hline & \multicolumn{2}{c}{ Mères } & \multicolumn{2}{c}{ Pères } \\
\cline { 2 - 5 } & $\boldsymbol{N}$ & $\%$ & $\boldsymbol{N}$ & $\%$ \\
\cline { 2 - 5 } & 54 & 90,0 & 6 & 10,0 \\
\hline Sollicite la participation parentale & 28 & 46,5 & 2 & 3,5 \\
\hline $\begin{array}{l}\text { Ne sollicite pas la participation } \\
\text { parentale }\end{array}$ & 26 & 43,0 & 4 & 6,5 \\
\hline N'accorde pas de choix & 4 & 6,5 & & \\
\hline Ignore et critique les suggestions & 4 & 6,5 & & \\
\hline Ne tient pas compte des opinions & 1 & 1,5 & & \\
\hline
\end{tabular}

\subsection{L'INVITATION AUX PARENTS À PARTICIPER AUX DÉCISIONS}

À la question 30, un peu moins de la moitié des parents de l'échantillon répondent que l'école les a invités à participer aux décisions concernant la situation-problème de leur enfant. Â l'inverse, un peu plus de la moitié d'entre eux croient que l'école ne les a pas invités à participer à de telles décisions: Ils nous ont informés des décisions qu'ils avaient prises avant notre arrivée aux rencontres (01).

Plus de la moitié des parents entretiennent la perception de ne pas être partie prenante dans le processus décisionnel. À leurs yeux, l'école leur impose même les décisions: J'ai dit ce que j'en pensais, mais ils n'ont pas voulu changer d'idée (46).

Parmi les parents qui ont la perception de ne pas être invités à participer aux décisions concernant leur enfant, deux sur cinq sont en litige avec l'école sur différentes questions, dont la première concerne la suspension scolaire: C'est eux qui ont décidé quoi faire, puis les trois quarts du temps, c'étaient des suspensions automatiques (97).

Cinq autres litiges avec les parents concernent la volonté de la garderie ou de l'école de renvoyer leur enfant: L'enfant change d'école en septembre. Cette décision a été prise par l'école (09); Quand je voulais inscrire mon enfant à l'école, la direction ne voulait même pas l'avoir (80).

Un troisième terrain de mésentente a trait au placement de l'enfant dans une école ou une classe spéciales, aux termes de la Section 20 de la Loi sur l'éducation de l'enfance en difficulté (Ministère de l'Éducation de l'Ontario, 2001b). À ce sujet, quelques parents s'y opposent fermement. Une mère a même dû consulter un psychologue privé dont l'intervention 
a permis de renverser la décision de placement déjà prise par l'école. Une autre a dû utiliser les services d'un médiateur lors des rencontres à l'école concernant les conditions de placement. En somme, l'ensemble de ces parents s'objectent à toutes formes d'exclusion socioscolaire de leur enfant: C'est comme s'ils voulaient se débarrasser de mon enfant (112).

De plus, près d'un parent sur deux rejette toute intervention à caractère majeur de la part de l'école. Pour cette raison, quelques-uns entretiennent un conflit ouvert avec la direction d'école que fréquente leur enfant. À titre d'exemple, un père de la classe moyenne n'accepte pas que l'école ait procédé à un signalement à la Société de l'aide à l'enfance (SAE) et une mère vivant en contexte de pauvreté se plaint qu'on ait procédé à un tel signalement sans son consentement. De telles interventions de la part de l'école entraînent souvent de très fortes réactions de la part des parents concernés: Je suis fâchée parce que la travailleuse sociale a téléphoné à la SAE. Elle a questionné mon enfant sans ma permission (12.1).

Selon un peu plus du tiers des parents, les règlements de l'école, instaurés en conformité avec le cadre légal face à la violence, sont souvent appliqués sans flexibilité ni souplesse. De plus, la manière qu'a l'école d'appliquer les règlements les confine souvent à un rôle passif qui génère une source de tension importante: Il faut qu'elle le mette dehors pour ne pas avoir de plaintes des autres parents. C'est un drôle de processus de prise de décisions, ça. Ça devient plus une action politique qu'une action pour aider l'enfant. C'est un manque de flexibilité de la part de l'institution (02).

Une autre source de tensions entre l'école et certains parents réside dans la pression qu'exerce le personnel scolaire pour que l'enfant soit médicamenté au Ritalin ${ }^{\circledR}$, malgré de fortes réticences de leur part. Toutefois, il semble que ces réticences concernent plus spécifiquement des parents de la classe moyenne: Ils ont essayé de m'obliger à le mettre sur le Ritalin ${ }^{\circledR}$ (102). L'invitation aux parents à participer aux décisions est présentée au tableau 4.8.

\subsection{L'INFLUENCE DES PARENTS SUR LES DÉCISIONS DÉJÀ PRISES PAR L'ÉCOLE}

La question 31 vise à cerner les perceptions que les parents entretiennent au sujet de leur influence sur les décisions déjà prises par l'école concernant la situation-problème de leur enfant. La forme de pouvoir que les parents exercent par rapport à l'école implique la capacité d'influencer les forces qui affectent la vie de l'enfant ou à développer une capacité à obtenir des effets désirés chez les autres (Pinderhughes, 1995). 
TABLEAU 4.8

L'invitation aux parents à participer aux décisions $(n=60)$

\begin{tabular}{lcccc}
\hline & \multicolumn{2}{c}{ Mères } & \multicolumn{2}{c}{ Pères } \\
\cline { 2 - 5 } & $N$ & $\%$ & $N$ & $\%$ \\
\cline { 2 - 5 } & 54 & 90,0 & 6 & 10,0 \\
\hline $\begin{array}{l}\text { Ne sont pas invités à participer } \\
\text { aux décisions }\end{array}$ & 30 & 50,0 & 2 & 3,5 \\
\hline $\begin{array}{l}\text { Ne se sentent pas partie prenante } \\
\text { des décisions }\end{array}$ & 30 & 50,0 & 2 & 3,5 \\
\hline Se font imposer les décisions & 27 & 45,0 & 1 & 1,5 \\
\hline $\begin{array}{l}\text { Rejettent les interventions } \\
\text { majeures de l'école }\end{array}$ & 27 & 45,0 & 1 & 1,5 \\
\hline $\begin{array}{l}\text { Sont invités à participer } \\
\text { aux décisions }\end{array}$ & 24 & 40,0 & 4 & 6,5 \\
\hline $\begin{array}{l}\text { Trouvent un manque de } \\
\text { flexibilité et de souplesse }\end{array}$ & 24 & 40,0 & 1 & 1,5 \\
\hline Vivent un litige & 23 & 38,0 & 2 & 3,5 \\
\hline $\begin{array}{l}\text { S'objectent à l'exclusion } \\
\text { socioscolaire }\end{array}$ & 23 & 38,0 & 2 & 3,5 \\
\hline$S^{\prime}$ opposent à la suspension scolaire & 17 & 28,0 & 2 & 3,5 \\
\hline S'opposent au Ritalin $^{\circledR}$ & 5 & 8,0 & & \\
\hline S'opposent au renvoi $^{\prime}$ & 5 & 8,0 & & \\
\hline S'opposent au placement $^{\text {Entretiennent un conflit ouvert }}$ & 4 & 6,5 & & \\
\hline
\end{tabular}

Les échanges avec l'école sont pour les parents des occasions de poser des gestes d'empowerment. L'empowerment se définit comme la perception d'exercer un contrôle réel sur les événements de sa vie (Rappaport, 1987). Il se concrétise dans les gestes d'adaptation, d'opposition et de résistance que posent les parents afin d'exercer une telle influence.

Le tiers des parents de l'échantillon ont la perception d'exercer une forme d'influence sur les décisions déjà prises par l'école concernant la situation-problème de leur enfant: Rien n'arrive sans mon accord (10); C'est moi qui ai la décision finale. Je crois que j'ai le pouvoir avec mes enfants, parce que ce sont mes enfants. C'est moi qui dois dire oui ou non, si je suis d'accord. C'est moi qui ai le dernier mot, parce que c'est moi la mère (112). 
On observe toutefois une distinction dans les réponses selon le milieu socioéconomique des familles. À ce titre, deux parents sur cinq des classes moyenne et moyenne-supérieure ont la perception d'exercer une telle influence comparativement au quart de ceux qui vivent en contexte de pauvreté.

Inversement, deux parents sur cinq ne considèrent pas détenir une influence sur les décisions déjà prises par l'école. Plus précisément, le tiers des parents des classes moyenne et moyenne-supérieure et les deux cinquièmes de ceux qui vivent en contexte de pauvreté répondent qu'ils n'exercent pas du tout d'influence. Une mère pauvre dit: C'est pas moi qui décide, c'est l'école, je n'ai pas le choix. Moi, c'est l'école. Je n'ai pas le choix, il faut accepter (105).

Quant au dernier tiers des parents, ils sont indécis: Je sais pas si c'est oui ou non. Comme des fois, ils vont le faire, puis des fois, ils le feront pas. Des fois, ils devraient peut-être toujours prendre nos opinions, puis peut-être faire... essayer ce qu'on dit un peu (102).

Certains expriment qu'ils n'ont pas à exercer une influence sur les décisions déjà prises par l'école parce qu'ils sont d'accord avec la position scolaire. Ils pourraient toutefois avoir éventuellement à le faire et, pour y parvenir, ils font confiance aux bonnes relations qu'ils entretiennent avec le personnel scolaire afin d'obtenir les résultats escomptés: Je crois que j'en aurais du poids. Oui, à cause de mon bon contact avec la directrice (110).

Par ailleurs, un parent sur dix se dit en confiance avec les décisions déjà prises par l'école. Le fait de ne pas éprouver le besoin d'exercer une influence sur de telles décisions peut résulter d'une reconnaissance de l'expertise du personnel scolaire: J'approuve l'aide que mon enfant a besoin (81); Le professeur de cette année a remarqué tout de suite qu'il y avait un problème et il a agi (106).

L'absence d'influence ne représente par un problème pour ces parents, car ils considèrent normal que l'école prenne seule les décisions. En ce sens, les milieux scolaire et familial sont perçus comme fournissant des contributions séparées (Epstein, 1992): Ce n'est pas mon rôle d'influencer l'école. J'ai pas fait d'études. En plus, c'est pas moi qui enseigne (106). L'influence des parents sur les décisions déjà prises par l'école selon le milieu socioéconomique est présentée au tableau 4.9. 
TABLEAU 4.9

L'influence des parents sur les décisions déjà prises par l'école selon le milieu socioéconomique $(n=60)$

\begin{tabular}{|c|c|c|c|c|}
\hline \multirow[b]{3}{*}{$\begin{array}{l}\text { Parents vivant en contexte } \\
\text { de pauvreté }\end{array}$} & \multicolumn{2}{|c|}{ Mères } & \multicolumn{2}{|c|}{ Pères } \\
\hline & $N$ & $\%$ & $N$ & $\%$ \\
\hline & 22 & 96,0 & 1 & 4,0 \\
\hline N'exercent pas d'influence & 8 & 35,0 & & \\
\hline Sont indécis & 8 & 35,0 & & \\
\hline Exercent une forme d'influence & 6 & 27,0 & 1 & 4,0 \\
\hline $\begin{array}{l}\text { Sont d'accord avec la position } \\
\text { de l'école }\end{array}$ & 3 & 13,0 & & \\
\hline $\begin{array}{l}\text { Ont confiance dans les décisions } \\
\text { déjà prises par l'école }\end{array}$ & 3 & 13,0 & 1 & 4,0 \\
\hline \multirow[t]{3}{*}{$\begin{array}{l}\text { Trouvent normal que l'école } \\
\text { décide seule }\end{array}$} & 3 & 13,0 & & \\
\hline & \multicolumn{2}{|c|}{ Mères } & \multicolumn{2}{|c|}{ Pères } \\
\hline & $N$ & $\%$ & $N$ & $\%$ \\
\hline $\begin{array}{l}\text { Parents des classes moyenne } \\
\text { et supérieure }\end{array}$ & 32 & 86,5 & 5 & 13,5 \\
\hline Exercent une forme d'influence & 13 & 35,0 & 1 & 3,0 \\
\hline N'exercent pas d'influence & 12 & 32,5 & 2 & 5,5 \\
\hline Sont indécis & 7 & 22,0 & 2 & 5,5 \\
\hline $\begin{array}{l}\text { Sont d'accord avec la position } \\
\text { de l'école }\end{array}$ & 2 & 5,5 & & \\
\hline $\begin{array}{l}\text { Ont confiance dans les décisions } \\
\text { déjà prises par l'école }\end{array}$ & 2 & 5,5 & & \\
\hline $\begin{array}{l}\text { Trouvent normal que l'école } \\
\text { décide seule }\end{array}$ & 1 & 3,0 & & \\
\hline
\end{tabular}

\subsection{EN RÉSUMÉ}

La distinction sur le plan du milieu socioéconomique des familles quant aux différents aspects de la participation parentale au processus de prise de décisions n'est pas aussi nette que celle qui s'observe au sujet de l'implication parentale dans la vie scolaire de leur enfant ou dans la communication entre les deux milieux. 
Sur le nombre de parents qui déclarent ne pas avoir été invités à participer à la recherche d'une solution (question 29), la moitié se situe dans la classe moyenne et moyenne-supérieure.

Quant au nombre de parents qui déclarent ne pas avoir été invités à participer aux décisions concernant leur enfant (question 30), les deux tiers sont regroupés dans les mêmes milieux socioéconomiques.

Finalement, sur le nombre de parents qui affirment ne pas détenir d'influence sur les décisions déjà prises par l'école (question 31), le tiers se situe dans les mêmes milieux socioéconomiques.

Cette distinction par milieu socioénonomique des familles ne se constate que par rapport à l'influence que les parents croient exercer sur les décisions déjà prises par l'école. Sur ce point, ceux qui vivent en contexte de pauvreté considèrent exercer moins d'influence que les autres. Seul le quart des parents vivant dans un tel contexte, comparativement à deux parents sur cinq des classes moyenne et moyenne-supérieure, croient exercer une telle influence. Pour chacun des autres aspects de la participation parentale au processus de prise de décisions, l'ensemble des parents se répartissent de façon équilibrée entre ces deux réponses.

\section{LA COLLABORATION SELON LE PROFIL DES PARENTS}

Lorsqu'on regroupe les parents de l'échantillon selon qu'ils répondent oui ou non aux trois questions portant sur la participation parentale au processus de prise de décisions, deux profils de parents se dégagent: ceux qui collaborent et ceux qui ne collaborent pas avec l'école. Les parents qui répondent positivement à ces trois questions sont classés dans le profil de collaboration. À l'inverse, ceux qui y répondent négativement font partie $\mathrm{du}$ profil de non-collaboration.

Le profil de collaboration reflète une communication positive entre les milieux scolaire et familial, telle que définie par Christenson et Sheridan (2001). Le profil de non-collaboration révèle plutôt une relation tendue pouvant mener au conflit.

Le profil de collaboration correspond à deux parents sur cinq de l'échantillon. Sur ce nombre, la moitié appartient aux classes moyenne et moyenne-supérieure et l'autre moitié vit en contexte de pauvreté. 
Quant au profil de non-collaboration, il correspond au tiers des parents de l'échantillon. Sur ce nombre, les deux tiers sont regroupés dans les classes moyenne et moyenne-supérieure et l'autre tiers vit en contexte de pauvreté.

En somme, les trois quarts des parents de l'échantillon correspondent à l'un ou l'autre de ces profils. De chacun de ceux-ci émergent des caractéristiques communes qui sont induites à partir des données d'entrevues. Selon le milieu socioéconomique des familles, les deux profils de parents fournissent un apport différent, tel qu'en fait foi l'analyse des données d'entrevue. Les parents des classes moyenne et moyenne-supérieure ont plutôt tendance à élaborer sur leur expérience de vie et à communiquer leurs perceptions à partir de notions abstraites, tandis que ceux qui vivent dans un contexte de pauvreté donnent des exemples de nature anecdotique tirés de leur vécu quotidien.

Le dernier tiers des parents de l'échantillon font preuve d'ambivalence puisqu'ils répondent à la fois positivement et négativement à l'une ou $l^{\prime}$ 'autre des trois questions se rapportant au processus de prise de décisions concernant leur enfant. De ce nombre, six sont regroupés dans les classes moyenne et moyenne-supérieure et trois vivent en contexte de pauvreté.

Un profil relativement flou émerge à partir de l'analyse des caractéristiques propres à ce regroupement, à savoir qu'aucun point commun ne semble se dégager ou ressortir des données d'entrevues. Dans la relation que ces parents entretiennent avec l'école, des éléments parfois positifs, parfois négatifs ressortent. Parmi les éléments positifs, ces parents semblent motivés à collaborer avec l'école. Par ailleurs, parmi les éléments négatifs, ils peuvent être en désaccord avec un enseignant, éprouver une réticence face à l'application d'une mesure disciplinaire ou vivre de la tension avec la direction d'école suite à des paroles échangées, etc.

Bref, l'ambivalence de ces parents illustre la complexité de la collaboration entre l'école et les parents et reflète la grande diversité dans les possibilités de réponses aux questions relatives à la participation des parents au processus de prise de décisions. Pour toutes ces raisons, il semble difficile, voire impossible de circonscrire un troisième profil regroupant les parents ambivalents. Nous n'avons toutefois pas ignoré les données $\mathrm{d}$ 'entrevues recueillies auprès de ces parents puisqu'elles ont fait l'objet d'analyses en fonction de l'âge de l'enfant, du niveau de scolarité des parents et du milieu socioéconomique de la famille.

Les deux profils de parents, soit ceux qui collaborent et ceux qui ne collaborent pas, nous aident à circonscrire l'état de leur relation avec l'école. Ils nous permettent également de répondre à l'une des questions 
secondaires de la présente recherche, à savoir quels éléments constituent un contexte de collaboration et quels sont ceux qui alimentent un contexte de non-collaboration, voire une relation conflictuelle entre l'école et les parents. De plus, l'analyse de ces deux profils de parents permet de faire ressortir des caractéristiques communes, qui sont induites à partir des données d'entrevues. Le profil de collaboration des parents selon le milieu socioéconomique est présenté au tableau 4.10.

TABLEAU 4.10

\section{Le profil de collaboration des parents selon le milieu socioéconomique $(n=60)$}

\begin{tabular}{|c|c|c|c|c|}
\hline \multirow[b]{3}{*}{$\begin{array}{l}\text { Parents vivant en contexte } \\
\text { de pauvreté }\end{array}$} & \multicolumn{2}{|c|}{ Mères } & \multicolumn{2}{|c|}{ Pères } \\
\hline & $N$ & $\%$ & $N$ & $\%$ \\
\hline & 22 & 96,0 & 1 & 4,0 \\
\hline Collaborent avec l'école & 11 & 48,0 & 1 & 4,0 \\
\hline Ne collaborent pas avec l'école & 8 & 35,0 & & \\
\hline \multirow[t]{3}{*}{ Font preuve d'ambivalence } & 3 & 13,0 & & \\
\hline & \multicolumn{2}{|c|}{ Mères } & \multicolumn{2}{|c|}{ Pères } \\
\hline & $N$ & $\%$ & $N$ & $\%$ \\
\hline $\begin{array}{l}\text { Parents des classes moyenne } \\
\text { et supérieure }\end{array}$ & 32 & 86,5 & 5 & 13,5 \\
\hline Font preuve d'ambivalence & 12 & 32,5 & 1 & 2,5 \\
\hline Collaborent avec l'école & 11 & 30,0 & 2 & 5,5 \\
\hline Ne collaborent pas avec l'école & 9 & 24,0 & 2 & 5,5 \\
\hline
\end{tabular}

\subsection{LE PROFIL DES PARENTS QUI COLLABORENT AVEC L'ÉCOLE}

Selon le milieu socioéconomique des familles, les parents qui collaborent avec l'école se distinguent les uns des autres, comme en font foi les données d'entrevue. Ceux des classes moyenne et moyenne-supérieure ont notamment tendance à élaborer sur leur expérience de vie et à communiquer leurs perceptions à partir de notions abstraites, tandis que ceux qui vivent dans un contexte de pauvreté donnent des exemples de nature anecdotique tirés de leur vécu quotidien. Parmi ces derniers, cinq prennent plus souvent la parole que les autres. Par ailleurs, cinq enfants de ce sous-échantillon fréquentent une école ou une classe spéciales à la suite d'un placement, aux termes de la Section 20 de la Loi sur l'éducation de l'enfance en difficulté (Ministère de l'Éducation de l'Ontario, 2001b). 
Quelles caractéristiques sont communes à ces 25 parents? À ce sujet, l'analyse des données d'entrevues révèle qu'en plus de se percevoir comme des partenaires à part entière, ils entretiennent une communication positive avec l'école, reconnaissent l'expertise du personnel scolaire, reconnaissent la complémentarité entre les milieux scolaire et familial, font des constats sur eux-mêmes ainsi que sur leur enfant, utilisent des services d'intervention sociale et perçoivent un travail concerté entre les membres du personnel scolaire. Le profil des parents qui collaborent avec l'école est présenté au tableau 4.11.

Tableau 4.11

Le profil des parents qui collaborent avec l'école $(n=25)$

\begin{tabular}{lcccc}
\hline & \multicolumn{2}{c}{ Mères } & \multicolumn{2}{c}{ Pères } \\
\cline { 2 - 5 } & $N$ & $\%$ & $N$ & $\%$ \\
\cline { 2 - 5 } & 21 & 84,0 & 4 & 16,0 \\
\hline $\begin{array}{l}\text { Entretiennent une communication } \\
\text { positive }\end{array}$ & 21 & 84,0 & 4 & 16,0 \\
\hline Reconnaissent l'expertise de l'école & 21 & 84,0 & 4 & 16,0 \\
\hline Reconnaissent leur complémentarité & 21 & 84,0 & 2 & 8,0 \\
\hline Utilisent des services d'intervention & 21 & 84,5 & 2 & 8,0 \\
sociale & 21 & 84,0 & 1 & 4,0 \\
\hline Font des constats sur eux-mêmes & 12 & 48,0 & 1 & 4,0 \\
\hline Perçoivent un travail concerté & 10 & 40,0 & 1 & 4,0 \\
\hline Font des constats sur leur enfant & & & &
\end{tabular}

\subsubsection{Une communication positive avec l'école}

Tous les parents qui collaborent avec l'école révèlent qu'ils entretiennent une communication positive avec l'école. Les perceptions des parents concernés contribuent à mieux cerner l'état de leur collaboration, selon la définition qu'en donnent Christenson et Sheridan (2001), à savoir un cadre d'échange dynamique où les deux milieux travaillent à l'atteinte de buts communs. À ce titre, une bonne collaboration se traduit par la mise en place d'une communication bidirectionnelle, enrichissante et productive, reposant elle-même sur une confiance et un soutien mutuels. Une telle collaboration se fonde également sur la prise de décisions communes concernant la situation-problème de l'enfant à l'école. 


\subsubsection{La reconnaissance de l'expertise de l'école}

Tous les parents du profil de collaboration reconnaissent l'expertise de l'école. Ces parents font confiance au personnel scolaire et à son analyse de la situation-problème: Eux autres, je peux leur faire confiance (84); Eux autres sont spécialisés dans leurs affaires. Puis, ils savent comment "dealer» avec des enfants difficiles. La façon qu'ils font leurs affaires. Puis, ils ont l'air vraiment qualifiés (112).

Certains parents de ce sous-échantillon apprécient l'ambiance qui règne à l'école, de même que les qualités du personnel scolaire: Le directeur est pas mal bon (06); L'école est sympathique, mais ferme et déterminée (10); Je dois dire que j'aime pratiquement tout ce qu'elle fait. Je la trouve très patiente et douce. Elle ne crie pas (13); Le personnel est strict et je trouve que c'est meilleur. L'école fait très bien. Je trouve que les interventions sont nécessaires (23); Elles sont toutes bien qualifiées. Aux réunions, ils cherchent toujours le positif (43); J'aime l'école que fréquente mon fils en ce moment (58); Ils prennent beaucoup soin de mon fils, ils ont beaucoup de patience (102); La travailleuse sociale, c'est une perle. Elle est vraiment là pour travailler avec ma fille (107).

La confiance des parents agit en faveur d'un travail concerté avec le personnel scolaire et vise l'atteinte de buts communs. En ce sens, ces parents se perçoivent comme des acteurs essentiels et se sentent appuyés dans leurs démarches: En tant que parent, on se dit qu'il faut faire confiance, mais ce n'est pas toujours facile de le faire. Il arrive quelquefois que c'est décourageant quand ça ne va pas. Mais on se sent toujours appuyé (20); Le directeur va dire à mon fils: Tu sais, c'est pas beau ce que t'as fait. Après ça, on dit à mon garçon: Ne copie pas les autres élèves (94).

Quelques parents apprécient les suggestions émanant du personnel scolaire: J'ai appelé quelquefois pour demander des suggestions. Puis je les prends, leurs suggestions (45); J'ai pris ce qui m'était suggéré (82).

Ces suggestions concernent les pratiques éducatives parentales permettant d'améliorer les comportements de leur enfant. Il s'agit d'un soutien direct de la part du personnel scolaire qui correspond à un aspect de la typologie d'Epstein (1995): L'école me donne vraiment des bons trucs avec lui pour nous en sortir (28); Avant, on utilisait toujours les mauvaises techniques. On utilise maintenant leurs techniques et ça nous a sauvés (45); Ils m'ont demandé de tenir mon bout à la maison autant qu'eux à l'école (86).

D'autres parents appuient les décisions déjà prises par l'école concernant leur enfant ainsi que son orientation. Les parents peuvent accepter les ressources d'aide proposées par l'école: Je fais confiance au personnel de l'école pour les décisions puisque c'est eux qui savent ce qui est mieux pour qu'il se comporte bien à l'école, puis après à la maison (37); C'est la directrice de l'autre 
école qui a offert une solution, la Section 20. Elle disait que son école n'avait pas les outils pour régler le problème et qu'il pourrait se faire suspendre pour n'importe quel de ses comportements. Nous avons suivi sa suggestion (45); Mais, l'école, ils travaillent très bien [...]. C'est pas moi qui décide, c'est l'école (105).

Également, quelques parents considèrent que la responsabilité de solutionner la situation-problème de l'enfant incombe à l'école. Dans cette optique, ils peuvent mettre en pratique des suggestions formulées par le personnel scolaire et accepter les ressources d'aide qui leur sont proposées: L'école s'en est occupée et il y a maintenant une travailleuse sociale (86); La directrice a suggéré que nous invitions des petits amis à la maison pour qu'il apprenne à socialiser avec des enfants de son âge. Nous avons invité des petits amis à la maison (114). Les dimensions de la reconnaissance de l'expertise de l'école sont présentés au tableau 4.12.

Tableau 4.12

Les dimensions de la reconnaissance de l'expertise de l'école $(n=25)$

\begin{tabular}{lcccc}
\hline & \multicolumn{2}{c}{ Mères } & \multicolumn{2}{c}{ Pères } \\
\cline { 2 - 5 } & $\boldsymbol{N}$ & $\%$ & $\boldsymbol{N}$ & $\%$ \\
\cline { 2 - 5 } & 21 & 84,0 & 4 & 16,0 \\
\hline Font confiance au personnel & 21 & 84,0 & 4 & 16,0 \\
\hline Apprécient l'ambiance de l'école & 19 & 76,0 & 3 & 12,0 \\
\hline $\begin{array}{l}\text { Apprécient les qualités du } \\
\text { personnel }\end{array}$ & 16 & 64,0 & 2 & 8,0 \\
\hline Se sentent des acteurs essentiels & 11 & 44,0 & 1 & 4,0 \\
\hline $\begin{array}{l}\text { Apprécient les suggestions } \\
\text { formulées }\end{array}$ & 7 & 28,0 & & \\
\hline $\begin{array}{l}\text { Appuient les décisions déjà prises } \\
\text { par l'école }\end{array}$ & 5 & 20,0 & & \\
\hline Mettent les suggestions en pratique & 3 & 12,0 & & \\
\hline Acceptent les ressources proposées & 2 & 8,0 & & \\
\hline
\end{tabular}

\subsubsection{La reconnaissance de la complémentarité entre l'école et les parents}

L'analyse des données d'entrevue révèle que la presque totalité des parents qui collaborent avec l'école reconnaissent la complémentarité entre les milieux scolaire et familial. De plus, les parents de ce sous-échantillon communiquent de manière franche et ouverte avec l'école. L'agenda 
scolaire est le moyen de communication privilégié en vue d'un travail conjoint visant l'atteinte de buts communs (Christenson et Sheridan, 2001). Ces parents s'en servent également pour assurer la constance dans leurs interventions quotidiennes: À chaque jour, on lit le commentaire dans l'agenda scolaire et on peut y répondre (45).

Selon ces parents, une réelle complémentarité s'opère par le biais de l'utilisation quotidienne de l'agenda scolaire. En signant l'agenda, ils attestent avoir pris connaissance de l'information transmise par le personnel enseignant. Par ce moyen de communication, ils peuvent rétroagir et faire part de leurs commentaires à l'enseignant: Avec les notes dans l'agenda scolaire, je suis au courant de tout et c'est bon que mon enfant sache qu'il y a une communication avec les parents qui donne lieu à des conséquences à la maison (62).

L'agenda scolaire devient le moyen privilégié pour une communication bidirectionnelle, enrichissante et productive: J'écris dans l'agenda scolaire si je suis à l'extérieur de la ville ou si quelque chose se passe dans la vie de mon fils (43).

Ce moyen permet d'instaurer une collaboration basée sur la confiance et le soutien mutuels (Christenson et Sheridan, 2001): J'écris si elle est très méchante, le matin avant qu'elle parte pour l'école: C'est de même qu'elle est aujourd'hui, fais attention. Comme ça, elle sait tout de suite de ne pas lui donner de chances (107).

Par conséquent, l'agenda scolaire contribue directement à l'amélioration de la situation-problème de l'enfant: Mon fils le sort maintenant de lui-même. Il en est très fier (20); Elle a fait une bêtise à l'école, elle le sait maintenant. Je suis même plus obligée de lui dire. Elle arrive à la maison avec son agenda scolaire, elle fait ce qu'elle a à faire. Puis, le lendemain, elle est fine à l'école (107).

Par ailleurs, la complémentarité entre les milieux scolaire et familial signifie qu'ils agissent dans le même sens. Il ne s'agit ni d'efforts isolés ni de contributions séparées, où l'un et l'autre agissent en vase clos, mais d'une confiance et d'un soutien mutuels: L'école sait qu'on travaille avec notre fils et que nous sommes disponibles. Ils me communiquent toujours la manière dont se sont passées les journées (37); On s'assoit, moi, le directeur et mon enfant. Puis, on parle de ce qui s'est passé, de ce qu'on va faire (94).

Également, pour certains parents, il est primordial de fonctionner main dans la main avec le personnel scolaire au bénéfice de leur enfant: Il dit: "Si on pousse la situation les deux ensemble, il va avoir hâte de revenir à l'école» (06); On travaille ensemble (10); On essaie de faire les mêmes règlements (112). 
Ces parents se déclarent intéressés à ce qui se passe à l'école afin de pouvoir échanger avec leur enfant: J'aime savoir ce qui se passe avec l'enfant à chaque jour pour que je puisse en parler à la maison (28); Faut aller à l'école pour voir ce qui arrive. Pour demander comment c'est arrivé (105).

De plus, des parents font preuve de cohérence avec l'école en imposant des conséquences à leur enfant en réponse à ses agissements en milieu scolaire: À la maison, mon fils est puni lorsqu'il fait des niaiseries à l'école. Il doit savoir qu'il a fait quelque chose de mal (23); I'ai dit de mettre les commentaires de façon journalière. À chaque fois qu'il faisait quelque chose de mal, de me le mettre dans l'agenda scolaire pour que je prenne les mesures nécessaires à la maison (86); S'il n'a pas eu une bonne journée, il perd sa télé le lendemain matin (114).

Les conséquences aux actes imposées par certains parents à la maison peuvent être adaptées en fonction de l'enfant et de la famille: À la maison, on donne une rétroaction à ce qui s'est passé. On peut lui demander de faire un dessin, d'écrire un petit mot (20); Je donne une récompense à mon fils au début de la semaine et une autre à la fin. Lorsque les récompenses sont trop éloignées, comme à la fin de chaque semaine, ça ne fonctionne pas bien. Il veut juste se rattraper (51); C'est moi qui ai décidé que si à la fin de la semaine, il y avait moins de points que l'enseignante voulait, il se ferait enlever un privilège ou, au contraire, on irait au McDonald (96).

Finalement, cinq parents vivant en contexte de pauvreté apprécient que le personnel scolaire respecte leurs opinions: L'école me respecte énormément. On respecte ce que je dis (10); Ils sont très respectueux (112).

Le respect qu'ils perçoivent les différencie des parents des classes moyenne et moyenne-supérieure. Ce respect se révèle une dimension fondamentale qui les motive à collaborer davantage avec l'école: Le directeur a dit une affaire, puis moi, j'ai dit une autre, puis on a réglé le problème. On a fait les deux, mais à moitié lui, à moitié moi (94).

Ces parents font ressortir la fierté qu'ils éprouvent à l'idée que le personnel scolaire reconnaît leur expertise en tant que parent. Il est à noter que quatre de ces cinq parents reçoivent des services d'intervention sociale et que le dernier parent consulte régulièrement un professionnel de la santé: Des choses que je suggère, je vois qu'ils le prennent au sérieux. Parce que je vois que des affaires que je suggère, ils vont le faire (96). Les dimensions de la reconnaissance de la complémentarité entre les milieux scolaire et familial sont présentées au tableau 4.13. 
TABLEAU 4.13

Les dimensions de la reconnaissance de la complémentarité entre les milieux scolaire et familial $(n=23)$

\begin{tabular}{lcccc}
\hline & \multicolumn{2}{c}{ Mères } & \multicolumn{2}{c}{ Pères } \\
\cline { 2 - 5 } & $N$ & $\%$ & $N$ & $\%$ \\
\cline { 2 - 5 } & 21 & 91,5 & 2 & 8,5 \\
\hline $\begin{array}{l}\text { Communiquent de manière } \\
\text { franche et ouverte }\end{array}$ & 21 & 91,5 & 2 & 8,5 \\
\hline Se soutiennent mutuellement & 11 & 48,0 & & \\
\hline Travaillent ensemble & 10 & 43,5 & & \\
\hline Font preuve de cohérence & 8 & 35,0 & \\
\hline $\begin{array}{l}\text { Adaptent les conséquences } \\
\text { aux actes }\end{array}$ & 5 & 22,0 & & \\
\hline $\begin{array}{l}\text { Apprécient le respect de leurs } \\
\text { opinions }\end{array}$ & 5 & 22,0 & & \\
\hline
\end{tabular}

\subsubsection{Les bienfaits des services d'intervention sociale}

La majorité des parents de ce sous-échantillon utilisent des services d'intervention sociale, à savoir qu'ils consultent des travailleurs sociaux et des psychologues, scolaires ou privés, ou encore des spécialistes de la relation d'aide œuvrant notamment dans des centres communautaires: Mon médecin, c'est lui qui m'avait référée. Ensuite, c'est le centre communautaire qui m'a aidée (13); C'est mieux depuis l'école: il y a la travailleuse sociale et des cours (20).

Ils consultent des professionnels qui leur sont disponibles grâce aux conseils scolaires, mais également grâce à leur ténacité: Je pense que si je n'avais pas été chercher de l'aide, je n'en aurais pas eu, mais ça n'aurait pas été à son avantage à lui [...]. Maintenant, on a le service d'orthophonie et d'ergothérapie. Alors, on dirait qu'on est soudainement entourés de toutes sortes de professionnels. Mais jusqu'à ce que l'école commence, on s'est sentis très seuls (20); Par rapport au TDAH*, j'ai rencontré le psychologue, mais je ne l'ai pas encore fait évaluer (23).

Ces professionnels remplissent un rôle central en appuyant les parents et, par conséquent, leur enfant. Cet appui profite également aux autres membres de la famille. En ce sens, l'intervention sociale les aide à faire face à des difficultés importantes: J'ai un avocat, mais avant de faire mes démarches

* Trouble du déficit de l'attention avec hyperactivité. 
avec lui, je veux avoir de l'aide de l'école, de la travailleuse sociale (102); Elle était là pour mon divorce (107); La travailleuse du centre communautaire était toujours avec moi quand j'allais à l'école (112).

Cette intervention permet d'appuyer le parent sur une base régulière: Oui, il y en a une travailleuse qui vient. Elle vient à chaque semaine, deux fois par semaine (94); Elle m'appelle aussi pour prendre des nouvelles de ma fille et me demander comment ça va (107).

Lorsque les parents se sentent ainsi soutenus et encouragés, en identifiant les succès au quotidien, ils tendent à reprendre courage. Un travail peut ensuite s'effectuer sur le plan des pratiques éducatives parentales: Elle nous a encouragés à continuer à faire ce que nous faisions de bien. Elle nous a donné son support (20); La travailleuse sociale est bien positive. Dans l'ensemble, lorsqu'on discute, c'est positif à la fin. Elle dit ce qui va bien (43).

Ces parents peuvent fournir les efforts nécessaires à l'acquisition de pratiques éducatives susceptibles d'aider leur enfant à surmonter la situation-problème à l'école. Ces pratiques se rapportent au contrôle comportemental: La travailleuse sociale a eu des suggestions pratiques comme la technique 1-2-3. Juste le mauvais renforcement que nous faisions à ses crises, quand on allait s'asseoir avec, c'était bon de nous dire ça (20).

On encourage le renforcement positif de l'enfant et de ses réussites: J'essaie toujours de le féliciter quand il fait quelque chose de bien. On m'a dit que c'est mieux de dire plus souvent ce qu'il a fait de bien que de renoter ce qu'il a fait de mal. C'est plus encourageant (86).

L'intervention sociale peut aider l'enfant à son développement et à l'apprentissage de compétences sociales: Ils l'ont suivi un peu plus avec la travailleuse sociale qui lui donne des outils (86).

Cette intervention a davantage de chances de porter fruit lorsqu'elle relève d'un travail d'équipe: Elle a travaillé directement avec mon fils, de concert avec les conseils du psychologue. Les visites chez le psychologue l'ont également énormément aidé (37).

Cette aide peut être également apportée en classe: Quelqu'un accompagne l'enfant dans la classe pour l'aider (49); Elle est une intervenante dans la classe qui sort mon fils de temps en temps pour le garder sous contrôle (81); Cette année, je le change d'école et la travailleuse sociale va retourner le voir. Espérons que ça va l'aider (82). 
L'intervention sociale auprès de l'enfant permet également de le sortir de son isolement: La travailleuse sociale aide beaucoup les enfants. Elle les prend individuellement. Je vais recommencer à lui faire revoir la travailleuse sociale pour mieux comprendre un peu l'histoire, pour l'aider aussi à comprendre les choses, qu'il n'est pas le seul comme ça (102).

Selon quelques mères de famille monoparentale, le manque de présence et d'influence masculine, qui peut représenter une difficulté accrue chez certains garçons, est pris en considération. L'intervention sociale réalisée par des hommes est vue comme un aspect positif: Mon fils consulte un homme, des fois, à l'école. Il aime bien être avec un homme (28); Elle a trouvé un Grand Frère à mon fils les fins de semaine. Ça lui fait de la compagnie, et il apprend à voir et à comprendre les comportements inacceptables (51).

Trois parents de la classe moyenne nuancent toutefois leurs propos positifs au sujet des bienfaits de l'utilisation de services d'intervention sociale. Ils désirent en bénéficier davantage ou maintenir un certain contrôle sur le soutien apporté à leur enfant: Je voudrais bien travailler seule avec mon fils sans impliquer des étrangers (58); J'aimerais probablement avoir plus d'aide. Je n'ai pas les moyens de me payer de l'aide externe (82); Nous aurions aimé avoir plus d'informations sur l'horaire des rencontres avec mon fils, et aussi sur les résultats (114). Les bienfaits de l'utilisation de services d'intervention sociale sont présentés au tableau 4.14.

TABLEAU 4.14

Les bienfaits de l'utilisation de services d'intervention sociale $(n=23)$

\begin{tabular}{lcccc}
\hline & \multicolumn{3}{c}{ Mères } & \multicolumn{2}{c}{ Pères } \\
\cline { 2 - 5 } & $N$ & $\%$ & $N$ & $\%$ \\
\cline { 2 - 5 } & 21 & 91,5 & 2 & 8,5 \\
\hline $\begin{array}{l}\text { Aident à faire face aux difficultés } \\
\text { quotidiennes }\end{array}$ & 13 & 56,5 & 1 & 4,0 \\
\hline Aident à reprendre courage & 11 & 48,0 & & \\
\hline $\begin{array}{l}\text { Aident au développement social } \\
\text { de l'enfant }\end{array}$ & 8 & 35,0 & & \\
\hline Brisent l'isolement de l'enfant & 5 & 22,0 & & \\
\hline $\begin{array}{l}\text { Apprécient les interventions } \\
\text { masculines }\end{array}$ & 4 & 17,0 & & \\
\hline $\begin{array}{l}\text { Désirent bénéficier davantage } \\
\text { des services }\end{array}$ & 3 & 12,0 & & \\
\hline $\begin{array}{l}\text { Désirent maintenir le contrôle } \\
\text { sur le soutien apporté }\end{array}$ & 3 & 12,0 & & \\
\hline
\end{tabular}




\subsubsection{Les constats des parents portant sur eux-mêmes}

Près de neuf parents sur dix du profil de collaboration font des constats importants sur eux-mêmes. Ces constats portent sur leurs pratiques éducatives parentales, sur leurs difficultés quotidiennes ou sur leur sensibilité en tant que parent.

L'ensemble des parents de ce sous-échantillon réfléchissent à ses pratiques éducatives parentales. Par rapport à ces pratiques, ils éprouvent généralement une sensation de perte de contrôle sur leur enfant: Des fois, c'est moi qui prends un «time-out» dans ma chambre. Il frappe sur la porte pour que je sorte. Je ne le contrôle pas. Heureusement, maintenant il est sur le Ritalin ${ }^{\circledR}$, parce qu'il est fort pour son âge (28).

À l'inverse, cette perception de perte de contrôle aide les parents à reconsidérer leurs pratiques éducatives: Je ne suis pas convaincue de ma façon d'aider. Je suis enseignante. Alors, je reconnais qu'il y a un problème. Il a toujours été très exigeant au niveau de mon attention (20).

Certains parents entretiennent un sentiment négatif à l'égard de leur compétence éducative: Depuis que l'enfant a 2 ans, j'ai eu plein de problèmes avec lui. Je suis allée à plein de groupes de parentage, de soutien, pour essayer plein de techniques qui n'ont pas fonctionné. Probablement que l'école me trouve trop permissive (51).

Dans ce contexte où les parents se voient en train de remettre en question leurs pratiques éducatives, ce cheminement leur apparaît jalonné d'essais et d'erreurs, d'efforts et de dépassements: Avant, on le manipulait parce que lui nous manipulait. On ne se trouvait pas gentils au début, mais l'enfant a besoin de se faire retirer des privilèges (45); Heureusement qu'il a son père qui est plus ferme que moi. Moi, je suis trop fatiguée le soir (114).

Du temps est nécessaire aux milieux scolaire et familial afin de réaliser certains constats: Je n'ai pas reconnu le problème avant qu'il aille à l'école. J'ai pensé qu'il était simplement actif. Il grimpait et il écrivait sur les murs. C'était moins pire ici puisqu'il n'était pas en interaction avec les autres (23); Il était gâté et c'est de ma faute. On voit un an plus tard qu'on a fait une erreur (58).

Un tel parcours parsemé de difficultés quotidiennes touche leur sensibilité de parent et peut éveiller en eux une sensation de souffrance: $C^{\prime}$ est difficile parce que c'est très émotionnel. Il nous faut rester au niveau objectif. On se pose toujours des questions quand son enfant a pleuré pendant plus d'une heure. Ce n'est pas facile pour un parent d'entendre ça (20); Des fois, c'est dur de savoir que ton fils est toujours en troubles (23); Je suis mélangée, puis bouleversée. 
Ça fait six ans que j'ai tout le temps des problèmes ici et là (102); Pendant ce temps-là, c'était vraiment stressant parce que j'allais à l'école. J'avais des problèmes avec elle. Puis, c'est pas ma seule enfant. Des fois, il m'appelait pour les trois. Puis, c'était un temps qui était vraiment difficile (112).

Cette souffrance se voit exacerbée si l'entrée en première année s'avère difficile: Envoyer un enfant à l'école devrait bien aller... C'est le rêve de tous les parents. Mais avec ma fille, c'est difficile (09).

Notamment, certains parents trouvent très ardu le passage du préscolaire au primaire: En première année, ç'a été le traumatisme. J'en avais des hallucinations. Coupez-moi les deux jambes, mais redonnez-moi mes enfants. Je les ai perdus à l'école. On était tellement ensemble avant, et maintenant, on ne l'est plus du tout (12.1).

De telles difficultés entraînent un quotidien à la fois difficile à vivre pour l'enfant et pour le parent: C'est parfois stressant. Les devoirs sont toujours très pénibles à faire le soir. Je n'ai pas toujours la patience qu'il faut. C'est le temps que ça finisse (37); En jardin, la suspension arrivait deux fois par semaine. C'est moi qui souffrais... parce que mon enfant ne voulait pas être à l'école (84).

Des signes d'amélioration chez l'enfant améliorent également le mieux-être des parents: Maintenant, son moral est meilleur. C'est moins pénible pour tout le monde (58); Mon fils est beaucoup plus de bonne humeur. Il est moins agressif le soir. Ça ne le dérange plus, le matin, d'aller à l'école. J'ai moins de misère à l'envoyer (82).

Ce mieux-être génère un sentiment de réussite et de reprise de contrôle chez les parents affrontant depuis longtemps une situation-problème qui semblait insurmontable. Cette perception permet de motiver davantage ces parents à travailler à améliorer la situation. En ce sens, elle les incite à persévérer et à poursuivre les efforts entrepris d'abord dans l'incertitude puis, de plus en plus, dans un contexte de succès: Très positif. Il a changé depuis qu'il va à la nouvelle école. Nous avons une nouvelle vie. Il ne faut surtout pas lâcher (45); Ça, je suis contente. C'est pas stressant. J'ai pas d'appel à chaque jour ou à chaque deux jours (112).

Des parents reconnaissent des étapes et des balises: Cette année, ça commence à se calmer. Je ne sais pas si c'est parce qu'il vieillit (23); Il y a eu un gros changement depuis le début de l'année. C'est plus facile de travailler avec lui. À l'école et à la garderie, ils ont remarqué la différence. À la maison, il y en a une, mais pas autant qu'à l'école. Mon fils a encore des épisodes. Ce n'est plus régulier comme c'était avant (37). 
Deux parents, afin d'améliorer les comportements de leur enfant, réfléchissent à une démarche permettant de mieux l'accompagner à travers ses embûches. Celle-ci les pousse à lui témoigner une plus grande affection: Quand il s'est calmé, je lui ai dit que je l'aimais. Je peux aussi prendre un toutou qu'il aime et je dis au toutou: "Ne le regarde pas, il est fâché et ça va te faire peur.» Mon fils veut alors son toutou pour lui donner des becs. Oui, je lui dis que moi, je vais toujours l'aimer: "une maman, ça aime toujours ses enfants» (43); J'essaie de le faire parler pour savoir ce qu'il a sur le cœur. Si je réussis, je le prends dans mes bras et je lui dis de ne pas s'inquiéter, que moi, je serai toujours là et qu'il n'y a pas de danger qu'il me perde. Je le console du mieux que je peux (86). Les constats des parents portant sur eux-mêmes sont présentés au tableau 4.15 .

TABleau 4.15

Les constats des parents portant sur eux-mêmes $(n=22)$

\begin{tabular}{lcccc}
\hline & \multicolumn{3}{c}{ Mères } & \multicolumn{2}{c}{ Pères } \\
\cline { 2 - 5 } & $N$ & $\%$ & $N$ & $\%$ \\
\cline { 2 - 5 } & 21 & 95,5 & 1 & 4,5 \\
\hline $\begin{array}{l}\text { Ont une sensation de perte } \\
\text { de contrôle sur l'enfant }\end{array}$ & 21 & 95,5 & 1 & 4,5 \\
\hline $\begin{array}{l}\text { Ont un sentiment négatif } \\
\text { de compétence éducative }\end{array}$ & 15 & 68,0 & \\
\hline Ont une sensation de souffrance & 11 & 50,0 & \\
\hline $\begin{array}{l}\text { Trouvent le quotidien difficile } \\
\text { à vivre }\end{array}$ & 5 & 23,0 & \\
\hline $\begin{array}{l}\text { Améliorent leur mieux-être } \\
\text { lorsque l'enfant s'améliore }\end{array}$ & 3 & 13,5 & & \\
\hline Identifient des étapes et des balises & 3 & 13,5 & & \\
\hline $\begin{array}{l}\text { Ont un sentiment de réussite } \\
\text { et de contrôle }\end{array}$ & 3 & 13,5 & \\
\hline $\begin{array}{l}\text { Font davantage de témoignages } \\
\text { d'affection à l'enfant }\end{array}$ & 2 & 9,0 & & \\
\hline
\end{tabular}

\subsubsection{Les constats des parents portant sur la situation-problème}

Les parents de ce sous-échantillon réalisent divers constats sur la situationproblème de leur enfant, de sa souffrance et des enjeux inhérents à l'intervention précoce. À ce sujet, la moitié d'entre eux mentionnent qu'il est important d'intervenir le plus tôt possible afin de minimiser les conséquences de la situation-problème: J'ai l'impression que plus on va avancer, 
plus il y aura des difficultés (20); Il faut l'attraper lorsqu'il est jeune puisqu'il n'en aura peut-être pas besoin lorsqu'il sera vieux. Le fait de prendre en main la situation tôt évitera des problèmes plus tard (23).

Ces parents craignent que, si la situation-problème n'est pas prise en main dans des limites de temps raisonnables, leur enfant ne bascule plus tard dans la délinquance: Je voulais pas que mon petit soit dans la délinquance (06); Peut-être à la longue, mon fils va avoir quelque chose de plus mal, de plus grave. Ils vont chercher quelque chose pour m'aider. Il ne fera pas comme son frère qui a été attrapé par la police (105).

Une mère a même peur que les comportements violents de son fils ne se retournent contre elle: Quand il va me dépasser, mon inquiétude, c'est que s'il continue ses comportements comme ça quand il sera plus grand, qu'il pense que peut-être sa mère sera rendue trop petite (96).

Cette appréhension incite les parents à faire des efforts supplémentaires pour éviter que la situation-problème actuelle ne dégénère: $O n$ essaie de faire des choses qui vont l'aider pour qu'il devienne un jeune qui ne se retrouvera pas dans une "gang» (43). Ces craintes pour l'avenir les motivent à collaborer avec l'école: C'est pour discipliner mes enfants, pour en faire des bons adultes un jour. Moi, je veux juste aider, parce que je veux que mon enfant ait une bonne «job», qu'il soit un bon adulte (112).

C'est parfois l'observation ou la découverte d'une souffrance vécue par l'enfant qui amène les parents à poser des gestes en faveur de sa socialisation: Les fins de semaine, il se retrouve toujours tout seul et il n'a pas d'amis dans le coin. Ça cause un peu de problèmes et c'est triste. C'est pour ça que pour sa fête, nous sommes allés à l'école parce que, sinon, il n'aurait eu personne à inviter (43).

Par ailleurs, certains parents se révèlent critiques face au système d'émulation utilisé par l'école: Avec leur système de récompenses, mon fils est souvent dans le rouge. Il a presque jamais le droit à rien. Il y a un vrai danger d'étiquetage et qu'il ne se fasse plus d'amis (114). Les constats des parents portant sur la situation-problème de leur enfant sont présentés au tableau 4.16. 
Tableau 4.16

\section{Les constats des parents portant sur la situation-problème de leur enfant $(n=11)$}

\begin{tabular}{lcccc}
\hline & \multicolumn{2}{c}{ Mères } & \multicolumn{2}{c}{ Pères } \\
\cline { 2 - 5 } & $N$ & $\%$ & $N$ & $\%$ \\
\cline { 2 - 5 } & 10 & 91,0 & 1 & 9,0 \\
\hline $\begin{array}{l}\text { Trouvent importante } \\
\text { l'intervention précoce }\end{array}$ & 10 & 91,0 & 1 & 9,0 \\
\hline $\begin{array}{l}\text { Craignent la délinquance } \\
\text { dans le futur }\end{array}$ & 6 & 54,5 & 1 & 9,0 \\
\hline $\begin{array}{l}\text { Constatent la souffrance } \\
\text { chez leur enfant }\end{array}$ & 3 & 27,0 & & \\
\hline $\begin{array}{l}\text { Posent des gestes en faveur } \\
\text { de sa socialisation }\end{array}$ & 3 & 27,0 & & \\
\hline $\begin{array}{l}\text { Sont critiques face au système } \\
\text { d'émulation }\end{array}$ & 2 & 18,0 & & \\
\hline Craint pour sa sécurité personnelle & 1 & 9,0 & & \\
\hline
\end{tabular}

\subsubsection{Un travail concerté entre tous les partenaires}

Un cinquième et dernier aspect reconnu par les parents des classes moyenne et moyenne-supérieure de ce sous-échantillon concerne le travail concerté entre tous les partenaires concernés par la situation-problème de l'enfant. Selon eux, cette concertation est essentielle à la collaboration. En ce sens, les parents mentionnent que le personnel scolaire n'hésite pas à mettre son expertise en commun avec la leur: À la garderie, ils ont travaillé fort avec la travailleuse sociale et l'enseignante (37); On travaille vraiment en équipe. L'enseignante me consulte sur tout ce qui concerne mon fils, elle me donne et me demande des conseils (86).

Le travail d'équipe s'effectue dans un esprit de collégialité visant à mobiliser tous les partenaires dans la recherche de solutions afin d'aider l'enfant à surmonter sa situation-problème. En somme, selon les propos de ces parents, le travail conjoint vise l'atteinte de buts communs. Ces éléments de soutien rejoignent la définition de la collaboration de Christenson et Sheridan (2001).

En outre, certains parents dont les enfants fréquentent une école ou une classe spéciales, suite à un placement aux termes de la Section 20 de la Loi sur l'éducation de l'enfance en difficulté (Ministère de l'Éducation de l'Ontario, 2001b), apprécient particulièrement le travail concerté entre les membres du personnel scolaire. Leur compétence est soulignée: Ils se sont occupés de lui. Ils savaient d'où il venait, son environnement. L'école où il était 
avant était dans un quartier défavorisé, donc c'était plus dur. En étant avertis, ils ont pris en considération et ont pris les méthodes nécessaires pour le corriger. Ils n'ont pas lâché, ils ne lâchent pas. Ils persévèrent tout le temps (86).

Des parents notent que le personnel scolaire fait preuve de disponibilité et d'engagement envers leur enfant: L'enseignante, qui est très occupée, nous téléphone aussi les fins de semaine. La directrice appuie toutes ses démarches (20); Si on veut les rencontrer, ils sont toujours disponibles (43); À l'autre école, avant la Section 20, deux professeurs lui ont fourni beaucoup d'aide, beaucoup de temps [...]. On peut parler au personnel de l'école en tout temps. Leur porte est toujours ouverte. On peut téléphoner en tout temps (45).

La compétence du personnel scolaire, en particulier celle de la direction d'école, est appréciée. Les parents soulignent sa vision positive, la mise en perspective des petites réussites, ainsi que la personnalisation des interventions destinées à leur enfant.

Des parents disent également apprécier les qualités personnelles du personnel scolaire, de même que leur capacité d'obtenir les services de professionnels autrement inaccessibles: C'est la directrice qui a obtenu la travailleuse sociale (20); La directrice m'a laissée parler avec un psychologue (23); Nous aimons beaucoup la directrice, qui est calme, claire, diplomate et met les choses en perspective (114). Les dimensions d'un travail concerté entre tous les partenaires sont présentées au tableau 4.17.

TABLEAU 4.17

\section{Les dimensions d'un travail concerté} entre tous les partenaires $(n=13)$

\begin{tabular}{lcccc}
\hline & \multicolumn{2}{c}{ Mères } & \multicolumn{2}{c}{ Pères } \\
\cline { 2 - 5 } & $N$ & $\%$ & $N$ & $\%$ \\
\cline { 2 - 5 } & 10 & 77,0 & 3 & 23,0 \\
\hline $\begin{array}{l}\text { Apprécient la mise en commun } \\
\text { de l'expertise }\end{array}$ & 10 & 77,0 & 3 & 23,0 \\
\hline $\begin{array}{l}\text { Apprécient la disponibilité } \\
\text { et l'engagement }\end{array}$ & 8 & 61,5 & 2 & 15,0 \\
\hline Reconnaissent la compétence & 5 & 38,5 & & \\
\hline $\begin{array}{l}\text { Apprécient les qualités } \\
\text { personnelles }\end{array}$ & 3 & 23,0 & & \\
\hline $\begin{array}{l}\text { Apprécient la capacité à obtenir } \\
\text { des services }\end{array}$ & 3 & 23,0 & & \\
\hline
\end{tabular}




\subsubsection{En résumé}

Les parents qui correspondent au profil de collaboration s'impliquent activement à l'école que fréquente leur enfant. Ils sont conscients de jouer un rôle important à l'intérieur d'un système où ils sont considérés comme partie prenante. Leur participation à une équipe de travail axée sur la recherche de solutions, la mobilisation de ressources et la mise en commun de l'expertise de tous les partenaires leur donnent des occasions de poser des gestes concrets. En tant qu'acteur essentiel, ils exercent un certain pouvoir qui contribue à créer un environnement scolaire et familial favorable à leur enfant.

Par ailleurs, les parents du profil de collaboration reconnaissent l'expertise de l'école, ses suggestions, reconnaissent la complémentarité des milieux scolaire et familial et communiquent de façon bidirectionnelle, enrichissante et productive par le biais de l'agenda scolaire. Certains parents de ce profil mettent l'accent sur le respect que le personnel scolaire leur témoigne, qui se manifeste par l'écoute de leurs commentaires et par la reconnaissance de leur compétence éducative parentale. De plus, ils font des constats importants sur leur situation familiale et sur la situation-problème de leur enfant. Dans la foulée des écrits sur le processus de demande d'aide, la première étape est la prise de conscience d'un problème ou du besoin (Cantin, 1998). Puis, en deuxième lieu, apparaît la décision d'agir en vue de trouver une solution au problème ou une réponse au besoin (Cantin, 1998, p. 36).

En somme, les parents de ce regroupement estiment qu'ils subissent des inconvénients marqués en relation avec la situation-problème de leur enfant à l'école. C'est pourquoi ils n'hésitent pas à recourir à des professionnels pour les aider à contrer, voire surmonter, leurs difficultés quotidiennes. À cet égard, ils bénéficient majoritairement de services d'intervention sociale, qui jouent un rôle déterminant dans la collaboration qu'ils entretiennent avec l'école. Une telle collaboration ne peut d'ailleurs pas s'établir sans un partenariat réel fondé sur une communication positive, et sur la confiance et le respect mutuels: On est des parents, vous autres, vous êtes les professeurs et directeurs. Puis, si on veut s'entendre, puis si il y a un problème, on devrait se respecter, puis essayer de parler ensemble pour voir ce qu'on peut faire (112).

Dans la présente section, nous avons observé des éléments constituant un contexte de collaboration, soit des facteurs de soutien sous-jacents, mais d'importants obstacles sont souvent à l'origine de tensions, voire de conflits, entre l'école et les parents faisant partie du profil de non-collaboration. 


\subsection{LE PROFIL DES PARENTS QUI NE COLLABORENT PAS AVEC L'ÉCOLE}

Dans la section précédente, l'analyse des données d'entrevues visait à circonscrire des éléments constituant un contexte de collaboration entre l'école et les parents, soit les facteurs de soutien qui lui sont sousjacents.

Cette analyse met notamment en lumière l'influence que les parents ont la perception d'exercer sur les décisions déjà prises par l'école concernant la situation-problème de leur enfant. Afin de mieux saisir les éléments constituant un contexte de non-collaboration, nous nous penchons dans la présente section sur les parents ayant les perceptions les plus négatives, soit sur les obstacles sous-jacents à la collaboration avec l'école, qui contribuent à rendre leur relation tendue, voire à la faire dégénérer en situation conflictuelle.

Quelles caractéristiques sont communes à ces 19 parents? Quels sont les éléments constituant un contexte de non-collaboration, qui alimentent leurs tensions et leur conflit avec l'école? À ce sujet, l'analyse des données d'entrevues révèle que ces parents doutent de leur influence sur les décisions déjà prises par l'école, éprouvent un sentiment d'impuissance face à la situation-problème de leur enfant à l'école, considèrent être la cible de qualificatifs et de jugements lourds de sens de la part du personnel scolaire, sentent une volonté de l'école d'exclure leur enfant, perçoivent un désengagement de l'école face à leur enfant, déplorent le manque d'écoute et d'ouverture et trouvent déficiente la communication avec le personnel scolaire. Le profil des parents qui ne collaborent pas avec l'école est présenté au tableau 4.18. 
Tableau 4.18

Le profil des parents qui ne collaborent pas avec l'école $(n=19)$

\begin{tabular}{lcccc}
\hline & \multicolumn{2}{c}{ Mères } & \multicolumn{2}{c}{ Pères } \\
\cline { 2 - 5 } & $N$ & $\%$ & $N$ & $\%$ \\
\cline { 2 - 5 } & 17 & 89,5 & 2 & 10,5 \\
\hline $\begin{array}{l}\text { Doutent de leur influence sur les } \\
\text { décisions déjà prises par l'école }\end{array}$ & 17 & 89,5 & 2 & 10,5 \\
\hline $\begin{array}{l}\text { Éprouvent un sentiment } \\
\text { d'impuissance }\end{array}$ & 13 & 68,5 & 1 & 5,5 \\
\hline $\begin{array}{l}\text { Sont la cible de qualificatifs et } \\
\text { de jugements lourds de sens }\end{array}$ & 12 & 63,0 & 1 & 5,5 \\
\hline $\begin{array}{l}\text { Sentent une volonté d'exclure } \\
\text { leur enfant }\end{array}$ & 10 & 52,5 & 1 & 5,5 \\
\hline $\begin{array}{l}\text { Perçoivent un désengagement } \\
\text { face à leur enfant }\end{array}$ & 10 & 52,5 & & \\
\hline $\begin{array}{l}\text { Déplorent le manque d'écoute } \\
\text { et d'ouverture }\end{array}$ & 8 & 42,0 & & \\
\hline $\begin{array}{l}\text { Trouvent la communication } \\
\text { déficiente }\end{array}$ & 7 & 37,0 & 1 & 5,5 \\
\hline
\end{tabular}

\subsubsection{L'absence d'influence sur les décisions déjà prises par l'école}

L'ensemble des parents du profil de non-collaboration doutent de leur influence sur les décisions déjà prises par l'école. Selon eux, le milieu scolaire ne les implique pas dans les décisions concernant leur enfant. À leurs yeux, de telles décisions devraient être prises en commun. De plus, ils considèrent que, sous une forme ou une autre, l'école les maintient dans un rôle passif, impose ses choix, applique ses règlements sans souplesse ou les force à se plier à ses exigences sans tenir compte des leurs. Ces éléments semblent constituer une source très importante de tensions entre les deux milieux.

Également, l'ensemble des parents se déclarent en conflit avec l'école parce qu'ils sont incapables de renverser des décisions déjà prises par l'école. Face à cette situation, certains d'entre eux dénoncent le caractère unilatéral du fonctionnement de l'institution scolaire: Ils nous ont dit qu'ils n'avaient pas le droit de faire ce qu'ils ont fait. Mais ça a été fait comme ça (44); L'école et moi devons travailler ensemble, mais ce n'est pas ce qui se passe (80); Ils l'ont entrainé debout contre ma volonté. Ils lui ont enlevé la doudou (109).

Les décisions déjà prises par l'école sans consultation des parents se rapportent aux suspensions scolaires imposées en relation avec le cadre légal face à la violence dans les écoles. Elles concernent également le 
placement de l'enfant dans une école et une classe spéciales, aux termes de la Section 20 de la Loi sur l'éducation de l'enfance en difficulté (Ministère de l'Éducation de l'Ontario, 2001b): Ils avaient des papiers. Ils ont dit: «Signez ici. On va faire la demande au conseil." Je n'étais pas vraiment d'accord, mais j'ai signé. J'avais pas le choix. C'était le plan d'intervention ou l'enfant ne va pas à l'école. Après, c'était soit la Section 20 ou rien du tout (108).

De même, la décision de renvoyer l'enfant de la garderie scolaire ou de l'école s'avère à l'origine de tensions importantes: Il était pour se faire renvoyer de la garderie. Ils voulaient un changement dès le lendemain. On a eu une rencontre avec la superviseure puis son patron à elle. Je pleurais, car un premier avis pour lui, puis un premier avis pour un autre enfant, c'est pas la même chose. J'ai commencé à les questionner tous les deux. Il n'a pas été "renvoyé» [suspendu] de la garderie (90).

Quant aux exigences formulées à l'encontre des parents, il s'agit des pressions importantes que l'école exerce sur eux afin que leur enfant soit médicamenté au Ritalin ${ }^{\circledR}$ : L'école dit: Il faut lui donner du Ritalin ${ }^{\circledR}$ ou on va continuer à le suspendre. Je n'étais pas d'accord du tout, du tout, du tout. Mais la raison que je lui en donne, c'est que je veux lui donner une chance à l'école (93).

Ces pressions peuvent nuire à la collaboration. Dans ces circonstances, le parent se perçoit parfois comme la victime de chantage et de menaces: La direction m'a menacée d'imposer trois jours. C'est comme du chantage. Je n'ai pas été impliquée sauf pour la suspension interne. Si je l'acceptais, je perdais la menace des trois jours de suspension (52).

Plus de la moitié des parents perçoivent qu'ils ne détiennent aucune influence sur les décisions déjà prises par l'école au sujet de leur enfant: Aucune. On n'a pas été écouté du tout (44); Aucune. Je n'aurais pas pu les faire changer d'idée. Le régime militaire: ils vous donnent des ordres, vous devez obéir (48); Aucune. Ils imposent. On doit obéir. Ce n'est pas ma définition de la collaboration (52); Aucune. Ils font pas mal plus ce qu'eux autres décident de faire. Ils font pas mal à leur tête. Ils ont des règlements, puis ils les suivent à la lettre (93).

Des parents avancent que malgré les efforts consentis pour accroître leur influence, ils n'y parviennent pas en raison des barrières organisationnelles évoquées par le personnel scolaire: Ils ne veulent pas accepter quoi que ce soit. C'est pas leur manière de faire les choses. Puis, ils ne sont pas intéressés à changer leur routine (97). 
Ils interprètent ces barrières comme de la résistance face au changement: J'en ai déjà discuté avec la directrice, des pratiques, dans la cour d'école. On $m^{\prime} a$ dit qu'on était pour regarder à ça. On ne m'en a jamais pas reparlé (99).

Malgré la perception de n'exercer aucune influence sur les décisions déjà prises par l'école, quelques parents obtiennent des gains à force d'insister, soit des services de professionnels de la part d'ergothérapeutes, d'orthothérapeutes et d'enseignants-ressources chargés de mettre en œuvre un Plan d'enseignement individualisé (PEI).

D'autres doivent toutefois recourir à des menaces afin d'obtenir de tels services: Après avoir fait une scène au conseil scolaire, oui, j'ai eu une petite influence (11.1); On a écrit une lettre au directeur. Après ça, on a dit qu'on était pour appeler au conseil scolaire s'il était pas prêt à nous aider, et c'est là que ça a changé un peu. Le directeur nous est revenu pour nous dire qu'il avait fait une demande pour avoir un psychologue pour notre fils, ce qu'on lui avait déjà demandé (97).

Sans détenir d'influence, deux parents déclarent qu'ils disposent d'une forme de contrôle: C'est moi qui contrôle le dosage du Ritalin ${ }^{\circledR}$ à l'école (48); J'ai dit: O.K., on va essayer parce que je suis vraiment tannée. Mais aux alentours du mois de mai, je vais enlever le Ritalin ${ }^{\circledR}$ (93).

En somme, les parents du profil de non-collaboration vivent une interaction difficile avec le personnel scolaire. L'analyse de leurs commentaires révèle qu'ils ont l'impression de n'exercer aucune influence sur les décisions déjà prises par l'école. Pourtant, leurs propos indiquent qu'ils disposent $d^{\prime}$ 'une certaine forme de contrôle qu'ils utilisent afin de minimiser les effets négatifs des décisions déjà prises par l'école, même sans avoir été consultés et sans avoir la possibilité de les renverser. Les dimensions de l'absence d'influence sur les décisions déjà prises par l'école sont présentées au tableau 4.19. 
TABLEAu 4.19

Les dimensions de l'absence d'influence sur les décisions déjà prises par l'école $(n=19)$

\begin{tabular}{lcccc}
\hline & \multicolumn{2}{c}{ Mères } & \multicolumn{2}{c}{ Pères } \\
\cline { 2 - 5 } & $N$ & $\%$ & $N$ & $\%$ \\
\cline { 2 - 5 } & 17 & 89,5 & 2 & 10,5 \\
\hline $\begin{array}{l}\text { Ne sont pas impliqués dans } \\
\text { les décisions }\end{array}$ & 17 & 89,5 & 2 & 10,5 \\
\hline $\begin{array}{l}\text { Se déclarent en conflit avec } \\
\text { l'école }\end{array}$ & 17 & 89,5 & 2 & 10,5 \\
\hline $\begin{array}{l}\text { Sont incapables de renverser } \\
\text { les décisions déjà prises }\end{array}$ & 17 & 89,5 & 2 & 10,5 \\
\hline $\begin{array}{l}\text { N'exercent aucune influence } \\
\text { sur les décisions }\end{array}$ & 13 & 68,5 & 2 & 5,5 \\
\hline $\begin{array}{l}\text { Ne parviennent pas à accroître } \\
\text { leur influence }\end{array}$ & 9 & 47,5 & & \\
\hline $\begin{array}{l}\text { Obtiennent des gains en insistant } \\
\text { ou par des menaces }\end{array}$ & 7 & 37,0 & & \\
\hline $\begin{array}{l}\text { Disposent d'une certaine forme } \\
\text { de contrôle }\end{array}$ & 2 & 5,5 & & \\
\hline
\end{tabular}

\subsubsection{Le sentiment d'impuissance des parents}

Les trois quarts des parents regroupés dans le profil de non-collaboration éprouvent un sentiment d'impuissance face à la situation-problème de leur enfant à l'école. Chez l'ensemble de ces parents, ce sentiment se traduit par une impression de dépassement, accompagnée de symptômes d'épuisement: Je vois ça comme quelque chose qui ne finira jamais! (44); Ça n'arrête jamais (83); Je pleurais parce que j'en pouvais plus. C'est toujours quelque chose qui va pas à la garderie, c'est toujours lui. J'arrive, il est toujours dans la salle de punitions. Toujours, toujours. On a eu une rencontre, je pleurais. J'étais plus capable de m'arrêter (90); Où est-ce que je m'en vais avec ça? (91); Mais je ne le sais plus, moi, je suis juste tannée (92); J'étais vraiment tanné. J'étais à bout. J'avais plus de ressources. Plus de place à aller. Je savais plus quoi faire! (93); Vraiment, c'est l'enfer! Je sais plus par quel bout prendre ça. J'aimerais que ça cesse. Mais j'ai comme l'impression que ça sera pas demain matin (99).

Ce sentiment d'impuissance peut également s'exprimer par de la déception et du découragement: Je suis déçue. Je me bats pour lui (11); Je suis très découragée par le système scolaire (44); J'étais découragée. J'en pouvais plus de la tension que m'imposait l'école. C'est difficile d'avoir raison sur quelqu'un qui a un diplôme (48). 
Les parents peuvent également ressentir de la solitude et de la frustration: Je me sens seule (09); Au début, j'étais un peu frustrée (12.1); J'étais très frustrée (48); Ça me frustre de voir qu'il se fait toujours blâmer (80); Je voyais un enfant de quatre ans complètement perdu. J'étais frustrée avec le personnel de l'école. C'était pour moi si difficile (108); Moi, j'étais frustrée. J'avais rien de bon à dire de l'école (111).

La tristesse, la honte et l'anxiété sont également des sentiments qu'éprouvent des parents: Ça me fait bien de la peine (11.1); Je dois constamment m'excuser parce que le petit est comme ça. À un moment donné, tu as honte. C'est pas le "fun» se faire dire que son enfant est violent, ou qu'il frappe. Je suis désolée pour les autres enfants. Ça me fait beaucoup de peine. J'en dors plus (91); À chaque fois qu'il passe à travers une épreuve, ça me fait beaucoup de peine (93).

De tels sentiments s'accompagnent parfois d'une perception de perte de contrôle face aux événements: J'aime autant mieux pas rencontrer d'autres parents, parce que je me dis que mon enfant les a peut-être frappés (91); Je peux pas contrôler l'environnement ailleurs. Je peux seulement le contrôler ici. Ça me traumatise. Il en est venu à me donner des coups de pied, des coups de poing, me mordre (109).

Quatre parents ressentent de l'agressivité face à l'absence d'empathie et de compréhension de la situation-problème de la part du personnel scolaire: Je suis sortie de là, je me sentais tellement agressive... (11.1); Une fois, la direction m'a dit de regarder les problèmes de mon fils avec les yeux d'un directeur d'école, pas avec ceux d'un parent. C'est complètement ridicule. Je n'ai pas aimé ça (52).

Ces divers sentiments ressentis par les parents témoignent de leur perte d'empowerment. Cette perte explique pourquoi certains d'entre eux peuvent se sentir incompétents dans l'éducation de leur enfant. Or, le sentiment de compétence éducative parentale est un facteur de soutien important dans la collaboration entre l'école et les parents (Eccles et Harold, 1993; Hoover-Dempsey, Bassler et Brissie, 1992). À l'inverse, l'absence de reconnaissance de la compétence éducative parentale par le personnel scolaire constitue un obstacle majeur à la collaboration.

Par ailleurs, l'absence d'une telle reconnaissance peut donner lieu, chez certains parents de ce sous-échantillon, à un questionnement qui constitue une ouverture face au changement: Ça nous affecte beaucoup. Tu sais, à un moment donné, tu penses comment tu agis. Je sais pas comment faire. Je le sais pas quoi faire (91); On devient démuni en tant que parent, parce que là, tu dis: "D'où est-ce qu'elle vient cette colère-là? " Là, tu regardes ton chez-toi (99). 
Selon Cantin (1998), la perception de la situation-problème ou sa prise de conscience constitue la première étape du processus de demande d'aide. À cet effet, les trois quarts des parents du profil de non-collaboration reçoivent des services $d$ 'intervention sociale, en ont déjà reçu dans le passé ou sont au début d'une démarche en ce sens. Si la majorité des parents recourent à de tels services, ils expriment toutefois des perceptions mitigées envers ceux-ci. Ainsi, certains parents avancent que le travailleur social est la seule personne avec laquelle ils peuvent échanger au sujet de leur vécu.

À l'inverse, une jeune mère remet en question la capacité de l'intervenante sociale à saisir la problématique avec laquelle elle est aux prises, même si la relation d'aide est établie sur de bonnes bases: La travailleuse sociale m'appelle à chaque semaine et nous discutons sur ce qui se passe à la maison et à l'école. Mais elle a tellement de misère à comprendre c'est quoi vraiment, avoir 23 ans et avoir un enfant de 6 ans qui a son père en prison (85). Les dimensions du sentiment d'impuissance des parents sont présentées au tableau 4.20.

TABLEAu 4.20

Les dimensions du sentiment d'impuissance des parents $(n=14)$

\begin{tabular}{lrccc}
\hline & \multicolumn{2}{c}{ Mères } & \multicolumn{2}{c}{ Pères } \\
\cline { 2 - 5 } & $\boldsymbol{N}$ & $\%$ & $\boldsymbol{N}$ & $\%$ \\
\cline { 2 - 5 } & 13 & 93,0 & 1 & 7,0 \\
\hline Sont dépassés et épuisés & 13 & 93,0 & 1 & 7,0 \\
\hline Sont déçus et découragés & 9 & 64,0 & 1 & 7,0 \\
\hline Se sentent seuls et frustrés & 7 & 50,0 & & \\
\hline $\begin{array}{l}\text { Ressentent de la tristesse, } \\
\text { de la honte et de l'anxiété }\end{array}$ & 6 & 43,0 & & \\
\hline $\begin{array}{l}\text { Perdent le contrôle face } \\
\text { aux événements }\end{array}$ & 5 & 36,0 & & \\
\hline $\begin{array}{l}\text { Sont agressifs face à l'absence } \\
\text { d'empathie }\end{array}$ & 4 & 28,5 & & \\
\hline $\begin{array}{l}\text { Se sentent incompétents dans } \\
\text { l'éducation de leur enfant }\end{array}$ & 3 & 21,5 & & \\
\hline $\begin{array}{l}\text { Se questionnent et sont ouverts } \\
\text { au changement }\end{array}$ & 2 & 14,0 & & \\
\hline $\begin{array}{l}\text { Échangent au sujet de leur vécu } \\
\text { Remet en question la capacité } \\
\text { de compréhension } \\
\text { de l'intervenante sociale }\end{array}$ & 2 & 14,0 & & \\
\hline
\end{tabular}




\subsubsection{Des jugements et des qualificatifs lourds de sens}

Les deux tiers des parents du profil de non-collaboration déclarent que le personnel scolaire porte des jugements ou leur attribue des qualificatifs lourds de sens. Ceux-ci s'appliquent à eux, à leur situation familiale ou à leur enfant. Les jugements et les qualificatifs concernent les principaux facteurs de risque inhérents aux comportements violents chez les enfants, tels que la séparation des conjoints, les mauvaises pratiques éducatives parentales, la violence conjugale, etc. Ces facteurs de risque deviennent une source d'étiquetage: C'était une erreur de leur raconter ma situation familiale, car ils ont classé mon fils. On s'attendait à ce qu'il agisse comme ça à cause de la séparation. Le directeur m'a même dit: "Il doit se passer quelque chose à la maison. Il se fait battre ou c'est peut-être la séparation» (11); Le directeur m'a dit que je devais battre mon enfant pour qu'il soit comme ça (44); Ils disent que c'est moi qui est trop tolérante avec lui (48).

L'abus sexuel est également ciblé: L'éducatrice m'avait accusée que vraiment, j'étais pas un bon parent. Elle a fait toutes sortes d'allusions. Elle m'avait demandé: «Est-ce que ton fils couche avec toi le soir? ? (111).

Ces jugements et qualificatifs peuvent également viser les traits de caractère de l'enfant. Celui-ci est alors identifié à la cause des perturbations vécues par le personnel scolaire et les pairs: La maîtresse $m^{\prime} a$ dit que mon enfant était dégueulasse. Elle m'a dit: "Je m'assois le matin en classe avec mon café et puis, je le vois sur sa chaise avec son doigt dans le nez, c'est dégueulasse! » [...]. C'est vraiment comme ça qu'elle me l'a dit, je n'exagère pas (11.1); On m'a dit que c'est le plus tannant des quatre classes de maternelle. Il a été malade deux jours, on m'a dit: "Ç'a été leurs deux meilleures journées. C'est de valeur à dire, c'est nos deux meilleures journées quand y est pas là» (91); Ils m'ont dit que mon fils était un «troublemaker». Que c'était lui la source du problème: «Il faut s'en occuper pour protéger les autres enfants» (97).

Afin de se protéger, voire de survivre dans un tel contexte, ces parents n'hésitent pas à recourir à leur tour à de tels stéréotypes. Par exemple, ils invoquent facilement le jeune âge de l'enseignant, son manque d'expérience, le fait qu'il ne soit pas un parent, pour le discréditer ou amoindrir l'effet de son étiquetage.

De plus, les parents réfutent les allégations qui s'avèrent sans fondement à leurs yeux, d'autant plus lorsqu'elles concernent l'enfant en très bas âge: Quand tu te fais dire par le directeur puis le professeur que ton fils de six ans va être un décrocheur plus tard... Tu peux pas, absolument pas dire ça. Quand l'école m'a dit ça, j'aurais dî les amener en cour (93); Un enfant de trois ans. Veux-tu bien me dire ce qu'il a fait de si mal à cet âge-là? Qu'est-ce qu'un enfant de trois ans est capable de faire de si mal? Trois ans! Là, je te parle pas d'un adolescent... Un enfant de trois ans. Il n'y a rien qu'un enfant de trois 
ans peut faire, qui est mauvais à ce point-là! (109). La nature des jugements et des qualificatifs lourds de sens posés par l'école sont présentés au tableau 4.21.

TABLEAu 4.21

La nature des jugements et les qualificatifs lourds de sens posés par l'école $(n=13)$

\begin{tabular}{lcccc}
\hline & \multicolumn{2}{c}{ Mères } & \multicolumn{3}{c}{ Pères } \\
\cline { 2 - 5 } & $N$ & $\%$ & $N$ & $\%$ \\
\cline { 2 - 5 } & 11 & 89,5 & 2 & 10,5 \\
\hline $\begin{array}{l}\text { Concernent les facteurs de risque } \\
\text { de la violence }\end{array}$ & 11 & 89,5 & 2 & 10,5 \\
\hline $\begin{array}{l}\text { Visent les traits de caractère } \\
\text { de l'enfant }\end{array}$ & 6 & 46,0 & & \\
\hline $\begin{array}{l}\text { Recourent à leur tour à des } \\
\text { stéréotypes }\end{array}$ & 5 & 38,5 & & \\
\hline $\begin{array}{l}\text { S'avèrent des allégations } \\
\text { sans fondement }\end{array}$ & 4 & 31,0 & & \\
\hline
\end{tabular}

\subsubsection{L'exclusion socioscolaire de l'enfant}

Près des deux tiers des parents qui correspondent au profil de noncollaboration sentent une volonté de l'école d'exclure leur enfant dès l'apparition des premiers comportements jugés inadéquats. Cette perception est corroborée par deux autres parents qui sont regroupés dans le profil de collaboration, mais qui ont une image négative de l'ancienne école que fréquentait leur enfant: C'est comme s'ils me menaçaient. Que si je lui donnais pas ça, elle pourrait pas rester à cette école-là (24); Il a été expulsé de la garderie en jardin. Ils voulaient s'en débarrasser. Donc, ils ont monté un bon dossier contre lui (43); Je ne suis pas contente où il est. Mais la direction voulait s'en débarrasser. Donc, le directeur m'a aidée à le changer d'école, mais c'était pour ne plus l'avoir avec eux (44); Quand je voulais inscrire mon fils à l'école, la direction ne voulait même pas l'avoir dans son école (80); Ils ont fait le PEI juste pour l'envoyer en dehors de l'école. La directrice était complètement certaine de pouvoir le renvoyer avec ça. J'ai toujours pensé qu'elle voulait se débarrasser de mon fils et de moi (108).

Pour certains parents, le fait de percevoir une volonté de l'école d'exclure leur enfant constitue un obstacle très important à la collaboration et à la poursuite des échanges. Ceux-ci perçoivent que toutes les actions du personnel scolaire sont dirigées vers l'atteinte de ce but. Face à l'application répétée de mesures disciplinaires sans consultation des parents, 
de même que l'insistance de l'école pour que les parents se plient à ses exigences, il devient ardu pour ces derniers de changer une telle perception lorsqu'elle est présente. Les tensions qui en découlent sont loin de faciliter une résolution de la situation-problème: Ils ont continué à insister pour que mon enfant prenne du Ritalin ${ }^{\circledR}$. Ç'a été fini. Je me suis dit: "Never mind», je le change de place (109).

Afin de contrecarrer la volonté d'exclusion socioscolaire de l'école, des parents ont recours à des services professionnels, tels qu'un psychologue privé. Ceux-ci craignent que les considérations administratives du milieu scolaire ne l'emportent sur le bien-être de leur enfant: Il a fallu qu'on aille se chercher un psychologue en privé. Il a fallu que le psychologue fasse les démarches pour s'assurer du transfert. Sinon, il serait allé directement en Section 20 (50); Ils voulaient le changer d'école tout de suite en partant. Puis, je me sentais pas à l'aise avec ça. Je suis allée en privé pour arrêter ça (111).

Deux autres mères, qui ne disposent pas des ressources financières nécessaires pour engager un tel professionnel, suggèrent l'idée qu'en pareille situation, un médiateur agisse pour faciliter la communication entre l'école et les parents. Les motifs de l'exclusion socioscolaire de l'enfant évoqués par l'école sont présentés au tableau 4.22.

Tableau 4.22

Les motifs de l'exclusion socioscolaire de l'enfant évoqués par l'école $(n=11)$

\begin{tabular}{lcccc}
\hline & \multicolumn{2}{c}{ Mères } & \multicolumn{2}{c}{ Pères } \\
\cline { 2 - 5 } & $\boldsymbol{N}$ & $\%$ & $\boldsymbol{N}$ & $\%$ \\
\cline { 2 - 5 } & 10 & 91,0 & 1 & 9,0 \\
\hline $\begin{array}{l}\text { Monte un dossier dès les premiers } \\
\text { comportements jugés inadéquats }\end{array}$ & 10 & 91,0 & 1 & 9,0 \\
\hline $\begin{array}{l}\text { Applique des mesures } \\
\text { disciplinaires répétitives }\end{array}$ & 7 & 63,5 & & \\
\hline $\begin{array}{l}\text { Tient d'abord compte de } \\
\text { considérations administratives }\end{array}$ & 2 & 18,0 & & \\
\hline
\end{tabular}

\subsubsection{Le désengagement de l'école}

La moitié des parents de ce sous-échantillon entretiennent la perception que l'école n'assume pas toujours ses responsabilités à l'égard de leur enfant: "Je n'ai pas pu vous appeler avant parce que j'avais la grippe.» Franchement! Elle enseignait, elle était à l'école! Elle pouvait m'appeler... (11.1); Je n'apprécie pas qu'ils ne soient pas toujours responsables avec les enfants (88). 
D'autres parents notent que le personnel scolaire a tendance à transférer facilement la responsabilité de l'enfant aux parents: La professeure a dit que c'était pas ses problèmes quand la journée est finie (90); Ils nous envoyaient des lettres qui nous disaient: "C'est pas à moi de faire ça. C'est votre enfant, occupez-vous-en!» (97).

Le désengagement de l'école est surtout perçu par les parents lorsqu'il est question du peu de services professionnels disponibles en milieu scolaire. Cette situation pousse les plus fortunés à se tourner vers le secteur privé pour obtenir de tels services. Fait à noter, les deux tiers des parents de ce profil ayant eu recours à des services professionnels en privé sont regroupés dans les classes moyenne et moyenne-supérieure: Il y a eu une évaluation qui a été faite, c'est tout. Il n'y a pas eu de suivi après ça. C'est à ce moment-là que je suis allée en privé (111). Les dimensions du désengagement de l'école à l'égard de l'enfant selon les parents sont présentées au tableau 4.23.

TABLEAU 4.23

Les dimensions du désengagement de l'école à l'égard de l'enfant $(n=10)$

\begin{tabular}{lcccc}
\hline & \multicolumn{2}{c}{ Mères } & \multicolumn{2}{c}{ Pères } \\
\cline { 2 - 5 } & $N$ & $\%$ & $N$ & $\%$ \\
\cline { 2 - 5 } & 10 & 100,0 & 0 & 0,0 \\
\hline $\begin{array}{l}\text { N'assume pas toujours } \\
\text { ses responsabilités }\end{array}$ & 10 & 100,0 & & \\
\hline $\begin{array}{l}\text { Ne dispose pas suffisamment } \\
\text { de services professionnels }\end{array}$ & 6 & 60,0 & & \\
\hline $\begin{array}{l}\text { Transfère facilement la } \\
\text { responsabilité aux parents }\end{array}$ & 4 & 40,0 & & \\
\hline
\end{tabular}

\subsubsection{Le manque d'écoute et d'ouverture du personnel scolaire}

Deux parents sur cinq de ce sous-échantillon entretiennent la perception que le personnel scolaire manque d'écoute et d'ouverture. Selon eux, certains de ses membres sont incapables d'accepter leurs commentaires, même si ceux-ci sont émis dans l'optique d'enclencher un processus de changement: Priver l'enfant des nécessités, ce n'est pas apprécié. On en a parlé et ça ne se règle pas. On n'a pas été écouté du tout (44); J'ai écrit une note avec un commentaire, on n'a pas eu de nouvelles (91); Toutes les demandes qu'on a fait et pour lesquelles on n'a pas eu de réponse: faire faire un retrait qu'ils n'ont réalisé qu'une fois, donner des avertissements avant chaque récréation, avoir un mentorat avec un plus grand, etc. (97). 
Le manque d'écoute et d'ouverture s'accompagne parfois d'un manque de considération face aux opinions exprimées par les parents: Je pense qu'ils se sentent obligés de m'écouter. Le grand boss du conseil scolaire les a appelés et leur a cogné sur les doigts. C'est juste parce qu'ils se sentent obligés (11.1).

Selon cinq parents, une autre raison pour laquelle l'école ignore leurs suggestions ou les solutions qu'ils proposent est qu'elles dérangent le mode de gestion scolaire même si, individuellement, chaque membre du personnel scolaire fait preuve de bonne volonté. En général, l'inertie décelée ne semble pas provenir des individus, mais du fonctionnement de l'institution scolaire: Ils m'ont dit que j'allais déranger la routine, que c'est pas une chose à faire, que c'est une perte de temps (97); Il y avait un mur entre l'école et nous, même si on était vraiment tous ouverts. Il n'y avait pas de système d'appui pour les parents, une personne qui expliquerait que c'est ça le système. Quelqu'un comme un médiateur (108); Oui, j'ai essayé de lui faire comprendre, mais je pense qu'elle était trop entêtée (109).

Des parents considèrent que le personnel scolaire ne reconnaît pas leur expertise parentale et qu'il nie leur capacité à interpréter les besoins de l'enfant: Elle me disait que c'était impossible. Je lui ai dit que je devais bien connaître mon fils (52); Le directeur, il ne me connaît pas et puis la maîtresse, elle ne me connaît pas non plus (92); Je l'ai aussi dit à son directeur, il ne me croit pas non plus. J'ai dit: "Oui, il veut une journée de vacances. Je le connais!" (97). Les dimensions du manque de d'écoute et d'ouverture du personnel scolaire sont présentées au tableau 4.24.

Tableau 4.24

Les dimensions du manque d'écoute et d'ouverture du personnel scolaire $(n=8)$

\begin{tabular}{lcccc}
\hline & \multicolumn{2}{c}{ Mères } & \multicolumn{2}{c}{ Pères } \\
\cline { 2 - 5 } & $N$ & $\%$ & $N$ & $\%$ \\
\cline { 2 - 5 } & 8 & 100,0 & 0 & 0,0 \\
\hline $\begin{array}{l}\text { Incapables d'accepter les } \\
\text { commentaires }\end{array}$ & 8 & 100,0 & & \\
\hline $\begin{array}{l}\text { Manquent de considérations } \\
\text { face aux opinions }\end{array}$ & 8 & 100,0 & \\
\hline $\begin{array}{l}\text { Ignorent les suggestions ou les } \\
\text { solutions proposées }\end{array}$ & 5 & 62,5 & \\
\hline $\begin{array}{l}\text { Manquent de reconnaissance de } \\
\text { l'expertise parentale }\end{array}$ & 4 & 50,0 & \\
\hline $\begin{array}{l}\text { Nient la capacité d'interpréter les } \\
\text { besoins de l'enfant }\end{array}$ & 4 & 50,0 & & \\
\hline
\end{tabular}




\subsubsection{La communication déficiente entre l'école et les parents}

Selon des parents de ce sous-échantillon, la communication permettant d'avoir une bonne collaboration avec l'école leur semble déficiente: Il a eu une bataille avec un ami, j'ai aucune idée de ce qu'ils ont fait. Ça fait deux jours, puis je le sais pas encore (92).

Cette communication leur semble déficiente parce qu'ils considèrent ne pas recevoir suffisamment d'informations au sujet de ce qui se passe à l'école: Il y a un gros manque de communication (83); J'aimerais ça savoir ce qui se passe à l'école. Qu'est-ce qu'il fait? Qu'est-ce qu'il fait pendant le dîner? Qu'est-ce qu'il fait dehors? J'aimerais ça savoir (92); Ils m'ont dit seulement ce qui était nécessaire. Pas plus. Donner pas beaucoup d'informations aux parents, c'est triste. Il nous faut de l'information précise (108).

Les parents qui n'arrivent pas à obtenir les éclaircissements demandés s'inquiètent de cette situation qui génère chez eux un sentiment de frustration: J'essayais d'expliquer au directeur, mais il n'a pas l'air de vouloir vraiment m'appeler. Il m'écrit juste des notes: Votre fils a fait ça (92); Il fallait que je coure pour pouvoir discuter avec quelqu'un. "Je suis là, moi! Je veux vous parler." Ces gens-là m'ont évitée au maximum. Il fallait vraiment que tu leur tires les vers du nez (109).

Alors que certains parents déplorent le manque d'information, à l'inverse, d'autres considèrent disproportionné le nombre d'appels téléphoniques effectués ou de notes écrites par l'école. Cette perception va dans le sens des écrits, à savoir que l'école a tendance à davantage communiquer avec les parents lorsque l'enfant éprouve des difficultés (Deslandes, 2001; Marcon, 1999): Je reçois trop d'appels de l'école. Elle téléphone de plus en plus souvent pour rien (11); L'année passée, c'était grave. Elle écrivait des choses à chaque jour. Elle m'a même écrit que mon fils se levait trop pour aiguiser son crayon (52); Avoir une meilleure communication avec moi, ça veut dire tout, à part de m'appeler à chaque jour pour dire que mon fils a lancé une efface et de venir le chercher! (85); Ils sont capables d'appeler pour une niaiserie (88); Je me suis fait appeler, je pense, trois ou quatre fois dans la même semaine par le directeur, comme quoi mon fils faisait ci ou ça (93); Ils appelaient pour tout, tout, tout. Ça n'avait plus de bon sens. Chaque petite niaiserie était rapportée. Dire pipi-caca à 4 ans. Tapez-moi pas sur les nerfs avec ça! (111).

Selon les parents, l'école procède parfois au filtrage de certaines informations. Cette pratique s'exerce lorsque le personnel scolaire a des raisons de croire que les parents utilisent des mesures jugées trop coercitives à l'endroit de l'enfant. Même s'il est parfois difficile d'avoir des certitudes et des preuves pouvant confirmer ses doutes, le personnel privilégie la sécurité de l'enfant au détriment d'une communication efficiente avec les parents: L'école ne me communique pas tous les mauvais comportements de ma 
fille. Ils craignent que je la tape. Taper, j'aime pas ça. Je ne veux plus le faire (09); L'école ne me tient pas au courant de toutes les actions parce qu'elle sait que je vais lui en parler et son enseignante l'aime beaucoup. Il lui a dit qu'il avait peur de moi (80). Les dimensions d'une communication déficiente avec l'école sont présentées au tableau 4.25.

TABleau 4.25

Les dimensions d'une communication déficiente avec l'école $(n=8)$

\begin{tabular}{lcccc}
\hline & \multicolumn{2}{c}{ Mères } & \multicolumn{2}{c}{ Pères } \\
\cline { 2 - 5 } & $\boldsymbol{N}$ & $\%$ & $\boldsymbol{N}$ & $\%$ \\
\cline { 2 - 5 } & 7 & 87,5 & 1 & 12,5 \\
\hline $\begin{array}{l}\text { Ne reçoivent pas suffisamment } \\
\text { d'informations }\end{array}$ & 7 & 87,5 & 1 & 12,5 \\
\hline $\begin{array}{l}\text { N'obtiennent pas les } \\
\text { éclaircissements demandés }\end{array}$ & 5 & 62,5 & & \\
\hline Manquent d'informations & 4 & 50,0 & & \\
\hline Reçoivent trop d'informations & 3 & 37,5 & & \\
\hline Reçoivent des informations filtrées & 3 & 37,5 & 1 & 12,5 \\
\hline
\end{tabular}

\subsubsection{En résumé}

Les échanges entre l'école et les parents de ce sous-échantillon traduisent des tensions où le conflit est toujours latent. La base d'un tel conflit se situe au plan de la participation parentale au processus de prise de décisions et à leur incapacité d'influencer celles qui sont déjà prises par l'école. Pour expliquer les divergences entre les deux milieux, Montandon et Perrenoud (1994) avancent que la perspective d'asymétrie de pouvoir, soit la force décisionnelle de l'école, agit au détriment de celle des parents. Or, les trois quarts des parents qui correspondent au profil de non-collaboration appartiennent aux classes moyenne et moyenne-supérieure. De plus, ils détiennent une formation universitaire qui les amène à faire preuve $\mathrm{d}^{\prime}$ esprit critique par rapport au milieu scolaire. Certains parents considèrent qu'ils sont l'objet de jugements et de qualificatifs lourds de sens, perçoivent un désengagement de l'école et sentent une volonté de l'institution d'exclure leur enfant.

Ces enjeux touchent majoritairement des parents d'enfants de six ans et moins des classes moyenne et moyenne-supérieure. Par conséquent, l'analyse des données d'entrevue nous amène à nous interroger sur la problématique de l'exclusion socioscolaire des très jeunes enfants ayant des comportements violents envers leurs pairs au préscolaire, et sur les 
ressources qu'elle requiert. Puisque le jeune âge des enfants représente un facteur de soutien bien documenté quant à l'implication des parents, il n'est pas étonnant que ceux-ci fassent preuve d'esprit critique face aux décisions déjà prises par l'école. D'où le cercle vicieux de la relation conflictuelle qu'ils entretiennent lors de divergences, notamment concernant la suspension scolaire, le placement dans une école et une classe spéciales aux termes de la Section 20 de la Loi sur l'éducation de l'enfance en difficulté (Ministère de l'Éducation de l'Ontario, 2001b) et au sujet du Ritalin ${ }^{\circledR}$. Bref, l'ensemble des parents qui correspondent au profil de non-collaboration se déclarent en conflit avec l'école parce qu'ils contestent les décisions déjà prises par l'école en s'opposant à toute forme d'exclusion socioscolaire et à toute intervention à caractère majeur.

Par ailleurs, la communication déficiente et le manque d'écoute ou d'ouverture de la part du personnel scolaire à l'endroit des commentaires et propositions formulés par les parents de ce sous-échantillon, constituent d'importants obstacles sous-jacents à la collaboration. Ces obstacles alimentent un contexte de non-collaboration, voire une relation conflictuelle entre les deux milieux.

En somme, les deux profils de parents permettent d'explorer les éléments qui constituent un contexte de collaboration et ceux qui alimentent un contexte de non-collaboration. Ces profils permettent également de mieux comprendre les facteurs de soutien et les obstacles sous-jacents à la collaboration. Par ailleurs, l'analyse des données d'entrevues permet de cibler d'autres aspects importants de la collaboration, notamment en ce qui a trait aux perceptions que les parents de l'échantillon entretiennent à l'endroit du personnel scolaire.

\section{LES PERCEPTIONS PARENTALES À L'ENDROIT DU PERSONNEL SCOLAIRE}

La majorité des parents de l'échantillon entretiennent à l'endroit du personnel scolaire des perceptions qui peuvent être à la fois positives et négatives. D'une part, les parents remarquent que certains membres du personnel font leur marque dans le processus de résolution de la situationproblème de leur enfant alors qu'à l'inverse, certains d'entre eux l'alimentent. Ces perceptions visent le personnel enseignant, la direction d'école et le personnel non enseignant: le personnel de la garderie et le personnel de soutien, tels le conducteur d'autobus, la secrétaire et la surveillante du dîner, pour ne nommer que ceux-là. Les perceptions parentales à l'endroit du personnel scolaire sont présentées au tableau 4.26. 
TABLeau 4.26

Les perceptions parentales à l'endroit du personnel scolaire $(n=60)$

\begin{tabular}{lcccc}
\hline & \multicolumn{2}{c}{ Mères } & \multicolumn{2}{c}{ Pères } \\
\cline { 2 - 5 } & $\boldsymbol{N}$ & $\%$ & $\boldsymbol{N}$ & $\%$ \\
\cline { 2 - 5 } & 54 & 90,0 & 6 & 10,0 \\
\hline $\begin{array}{l}\text { Entretiennent des perceptions } \\
\text { positives }\end{array}$ & 40 & 66,0 & 3 & 5,0 \\
\hline $\begin{array}{l}\text { Entretiennent des perceptions } \\
\text { négatives }\end{array}$ & 29 & 48,0 & 2 & 3,5 \\
\hline
\end{tabular}

\subsection{DES MEMBRES DU PERSONNEL SCOLAIRE QUI FONT LEUR MARQUE}

Près des trois quarts des parents de l'échantillon entretiennent des perceptions positives à l'endroit des membres du personnel scolaire. La disponibilité et l'ouverture dont ils font preuve ainsi que les interventions personnalisées destinées à leur enfant constituent l'essence de cette perception positive.

Les parents ont émis un commentaire favorable à l'endroit d'au moins un des membres du personnel scolaire de l'école, qui leur semble faire sa marque dans le processus de résolution de la situation-problème de leur enfant. Les perceptions parentales positives à l'endroit du personnel scolaire sont présentées au tableau 4.27.

Tableau 4.27

Les perceptions parentales positives à l'endroit du personnel scolaire $(n=43)$

\begin{tabular}{lcccc}
\hline & \multicolumn{2}{c}{ Mères } & \multicolumn{2}{c}{ Pères } \\
\cline { 2 - 5 } & $\boldsymbol{N}$ & $\%$ & $\boldsymbol{N}$ & $\%$ \\
\cline { 2 - 5 } & 40 & 93,0 & 3 & 7,0 \\
\hline Apprécient le personnel enseignant & 27 & 63,0 & 2 & 4,5 \\
\hline Apprécient la direction d'école & 18 & 42,0 & 1 & 2,0 \\
\hline $\begin{array}{l}\text { Apprécient le personnel non } \\
\text { enseignant }\end{array}$ & 14 & 32,5 & & \\
\hline
\end{tabular}




\subsubsection{Le personnel enseignant}

Plus des deux tiers des parents qui entretiennent des perceptions positives à l'endroit du personnel enseignant reconnaissent des qualités personnelles à certains membres: Elle est bien encourageante (05); L'enseignante est très gentille (28); Elle est super (32); Elle voit au bien-être des enfants (38); L'enseignante parle toujours de façon calme (37); Elle a une patience d'ange. Elle est très positive (43); Elle l'aime beaucoup (80); Elle est très gentille (105); L'enseignante est très fine (114).

Plus de la moitié des parents de ce sous-échantillon apprécient les membres du personnel enseignant qui se montrent disponibles et ouverts à l'idée d'expérimenter des suggestions de fonctionnement avec leur enfant: J'ai demandé à l'enseignante qu'après l'école, elle me montre les devoirs à faire et ce qu'il doit amener. Elle a collaboré (24); J'ai parlé à l'enseignante concernant les encouragements à lui donner. Après ça, j'ai remarqué qu'elle a fait un effort (47); Son enseignante voulait avoir mes suggestions et qu'est-ce que j'en pensais. Elle me donnait des situations qu'elle faisait en classe, puis elle me demandait qu'est-ce qu'elle pouvait faire ou c'était quoi qu'elle pourrait lui dire de faire (96).

Deux parents sur cinq apprécient les interventions personnalisées qui sont destinées à leur enfant: Elle aide autant les enfants qui sont plus forts que les enfants qui ont plus de difficulté. L'année passée, elle en avait cinq six qui étaient comme ça et elle a travaillé très fort avec toute la "gang». Elle fait beaucoup de choses pour essayer de les organiser, de les encourager. Ça l'a beaucoup aidé (05); Elle a trouvé une assistante qui a donné beaucoup d'attention à ma fille (09); Elle ressortait les aspects positifs de mon fils. L'an passé, l'enseignante lui disait toujours: "Tu as bien fait ça» (90).

Pour beaucoup de parents, l'enseignant représente l'interlocuteur privilégié permettant d'échanger sur la réalité quotidienne que vit leur enfant au préscolaire et au primaire: L'enseignante a beaucoup de pouvoir, car la qualité de la soirée de mon enfant dépend beaucoup d'elle et de ses commentaires dans l'agenda scolaire (15).

Devant les efforts déployés par certains enseignants afin d'améliorer la situation-problème de leur enfant à l'école, des parents se disent conscients qu'il s'agit d'un métier exigeant: Je sais que je suis exigeante avec l'enseignante. Puis, des fois, je me dis que si elle a choisi ce métier-là, à quelque part, c'est parce qu'elle aime les enfants (24). La nature des perceptions parentales positives à l'endroit du personnel enseignant est présentée au tableau 4.28. 
TABLEAU 4.28

\section{La nature des perceptions parentales positives à l'endroit du personnel enseignant $(n=43)$}

\begin{tabular}{lcccc}
\hline & \multicolumn{2}{c}{ Mères } & \multicolumn{2}{c}{ Pères } \\
\cline { 2 - 5 } & $N$ & $\%$ & 3 & $\%$ \\
\cline { 2 - 5 } & 40 & 93,0 & 1 & 2,0 \\
\hline $\begin{array}{l}\text { Reconnaissent les qualités } \\
\text { personnelles }\end{array}$ & 28 & 65,0 & 1 & 2,5 \\
\hline $\begin{array}{l}\text { Apprécient la disponibilité } \\
\text { et l'ouverture }\end{array}$ & 23 & 53,5 & & \\
\hline $\begin{array}{l}\text { Apprécient les interventions } \\
\text { personnalisées }\end{array}$ & 17 & 39,5 & & \\
\hline $\begin{array}{l}\text { Apprécient l'échange } \\
\text { sur la réalité quotidienne }\end{array}$ & 7 & 16,0 & & \\
\hline $\begin{array}{l}\text { Apprécient les efforts pour } \\
\text { améliorer la situation-problème }\end{array}$ & 4 & 9,5 & & \\
\hline
\end{tabular}

\subsubsection{La direction d'école}

Près de la moitié des parents qui entretiennent des perceptions positives à l'endroit de la direction d'école déclarent que c'est en raison du temps qu'elle leur consacre ainsi qu'à leur enfant: Le directeur s'occupe beaucoup de mon fils (06); La directrice prend le temps d'intervenir (10); Elle prend le temps. C'est qu'elle va le faire parler de ses émotions. On a un très bon contact. J'ai des longues discussions avec elle (90).

Près de la moitié des parents de ce sous-échantillon reconnaissent avoir reçu des suggestions appropriées de la part de la direction d'école, qui leur semble alors pleinement jouer son rôle de partenaire dans le processus de résolution de la situation-problème. Les suggestions les plus couramment formulées portent sur des consultations auprès de professionnels de la santé, des services de consultation psychologique ou d'intervention sociale. La direction d'école évoque également la possibilité de placer l'enfant dans une école ou une classe spéciale, aux termes de la Section 20 de la Loi sur l'éducation de l'enfance en difficulté (Ministère de l'Éducation de l'Ontario, 2001b). En plus de leur prodiguer des conseils ou des suggestions de lecture, la direction d'école peut suggérer aux parents d'adhérer à un groupe de parentage. Elle peut aussi suggérer que l'enfant participe à des programmes de développement des compétences sociales: Il m'a demandé de laisser mon enfant participer à un projet-pilote et j'ai évidemment dit oui (12). 
Ces mêmes parents notent que la direction d'école fait la part des choses: La directrice de l'année passée était super. Elle écoutait les deux bords des histoires (32); La directrice a pris en considération qu'il n'avait pas eu son médicament (90).

Des règlements clairs, de même qu'un code de conduite bien compris par près du tiers des parents, les rassurent tout en leur garantissant que les mesures disciplinaires seront appliquées de manière juste et équitable pour tous les enfants: C'est un nouveau système que la direction a installé. Quand les enfants ne respectent pas le code de vie, on est avisé par une fiche bleue. L'enfant et les parents doivent signer la fiche. Puis, il y a une discussion avec le directeur et le professeur. C'est seulement au deuxième avertissement qu'il y a une suspension (106).

Un dernier élément particulièrement apprécié de la part d'un parent sur cinq concerne le fait d'être consulté et impliqué par la direction d'école dans le processus décisionnel concernant son enfant à l'école: Avant d'appliquer une décision, la direction m'appelle pour me consulter. Ils essaient de fonctionner en m'impliquant. En me disant: »Regarde, ça c'est la situation. $Q u^{\prime} e s t-c e$ qu'on fait pour régler ça?» (02); La directrice disait que c'était l'école de la communauté. Elle voyait l'école non pas comme étant la sienne, mais celle des élèves et de leurs parents. Pour ça, c'était une perle. Quand j'ai vu cette directrice-là, le vocabulaire qu'elle avait, j'ai dit que c'était formidable (08). La nature des perceptions parentales positives à l'endroit de la direction d'école est présentée au tableau 4.29.

TABLEAu 4.29

\section{La nature des perceptions parentales positives à l'endroit de la direction d'école $(n=43)$}

\begin{tabular}{lcccc}
\hline & \multicolumn{2}{c}{ Mères } & \multicolumn{2}{c}{ Pères } \\
\cline { 2 - 5 } & $N$ & $\%$ & $N$ & $\%$ \\
\cline { 2 - 5 } & 40 & 93,0 & 3 & 7,0 \\
\hline $\begin{array}{l}\text { Apprécient le temps consacré } \\
\text { au parent et à l'enfant }\end{array}$ & 18 & 42,0 & 1 & 2,5 \\
\hline $\begin{array}{l}\text { Reconnaissent avoir reçu des } \\
\text { suggestions appropriées }\end{array}$ & 18 & 42,0 & 1 & 2,5 \\
\hline $\begin{array}{l}\text { Soulignent la capacité de faire } \\
\text { la part des choses }\end{array}$ & 18 & 42,0 & 1 & 2,5 \\
\hline $\begin{array}{l}\text { Apprécient l'application juste et } \\
\text { équitable des mesures disciplinaires }\end{array}$ & 13 & 30,0 & & \\
\hline $\begin{array}{l}\text { Apprécient être consultés et } \\
\text { impliqués dans les décisions }\end{array}$ & 8 & 18,5 & & \\
\hline
\end{tabular}




\subsubsection{Le personnel non enseignant}

Cinq parents de ce sous-échantillon entretiennent des perceptions positives à l'endroit du personnel non enseignant de l'école. Ces perceptions se rapportent aux liens étroits qui les unissent à certains membres du personnel de soutien. Pour cette raison, ils peuvent tirer avantage de la connaissance que ceux-ci ont du fonctionnement de l'école: C'est dommage à dire. Je pense ça parce que je suis bénévole à l'école. J'appelle la secrétaire, elle me connaît. Elle m'aide. Je connais les rouages (90).

Cet avantage s'applique également aux autres membres du personnel scolaire: Je m'informe auprès de l'enseignante comment ça se passe avec mon enfant puisque je suis gardienne à l'école lors du dîner. Ce n'est pas aussi souvent que j'aimerais, mais la directrice de l'école m'en parle toujours lorsqu'elle me voit (23).

\subsection{DES MEMBRES DU PERSONNEL SCOLAIRE QUI ALIMENTENT LA SITUATION-PROBLÈME}

Si près des trois quarts des parents de l'échantillon entretiennent des perceptions positives à l'endroit des membres du personnel scolaire, la moitié d'entre eux entretiennent néanmoins des perceptions négatives. Ces perceptions se rapportent aux méthodes éducatives, soit à la gestion de classe et aux modes de fonctionnement de l'école. Par ailleurs, des traits de personnalité et des défauts personnels sont également soulignés comme étant propices à alimenter la situation-problème de leur enfant. Les perceptions parentales négatives à l'endroit du personnel scolaire sont présentées au tableau 4.30 .

TABLEAU 4.30

Les perceptions parentales négatives à l'endroit du personnel scolaire $(n=31)$

\begin{tabular}{lcccc}
\hline & \multicolumn{2}{c}{ Mères } & \multicolumn{2}{c}{ Pères } \\
\cline { 2 - 5 } & $N$ & $\%$ & $N$ & $\%$ \\
\cline { 2 - 5 } & 29 & 93,5 & 2 & 6,5 \\
\hline $\begin{array}{l}\text { N'apprécient pas le personnel } \\
\text { enseignant }\end{array}$ & 19 & 64,5 & 1 & 3,0 \\
\hline $\begin{array}{l}\text { N'apprécient pas la direction } \\
\text { d'école }\end{array}$ & 14 & 45,0 & 1 & 3,0 \\
\hline $\begin{array}{l}\text { N'apprécient pas le personnel } \\
\text { non enseignant }\end{array}$ & 4 & 13,0 & & \\
\hline
\end{tabular}




\subsubsection{Le personnel enseignant}

Les deux tiers des perceptions négatives entretenues par les parents à l'endroit du personnel enseignant concernent la gestion de classe. Ces parents n'apprécient pas particulièrement les cas où celle-ci est jugée déficiente ou inappropriée: Dans la classe, il y avait cinq enfants comme mon fils. L'enseignante les a mis les cinq à la même table. Ça ne marchait pas. Après, elle a placé mon fils au fond de la classe. Puis, elle le prenait par le bras pour le sortir dans le passage. Il ne voulait pas sortir. Il se débattait. L'enseignante a alors dit qu'elle n'avait plus le contrôle sur lui (48); C'était la seule prof qui ne pouvait pas mettre tous les enfants en rang, avant d'entrer dans l'école. Les enfants étaient comme des petits poussins qui couraient partout (108); Le prof n'a pas les capacités de gérer la classe (109).

Ce type de difficultés semble surtout présent chez de jeunes enseignants ou chez ceux qui sont au début de leur carrière: Plus les enseignantes étaient jeunes, on dirait que plus elles avaient des problèmes. C'était comme si elles avaient de la difficulté à contrôler mon fils (91).

Plus du tiers des parents de ce sous-échantillon critiquent le manque de flexibilité et de souplesse du personnel enseignant: Des fois, il avait des réactions un peu trop sévères envers lui (01); Son enseignante est très rigide... Je l'avais rencontrée avant que les classes commencent en lui expliquant ses besoins, puis ses difficultés. Selon elle, être juste, ça voulait dire de traiter tous les enfants de la même manière. Mais pour moi, être juste, ça veut dire d'être capable de s'adapter à chaque enfant (04); J'ai toujours dit qu'à sa première année, c'était plate pour mon fils. Pas le droit de bouger et de parler. Il faut écouter et se concentrer. Ça marche pas avec lui! Moi, je peux le faire concentrer sur n'importe quoi parce que je joue avec lui, mais pas l'enseignante. [...] Elle n'a jamais essayé de trouver de nouvelles méthodes (48).

Pour le tiers de ces parents, les enseignants ne comprennent pas leur enfant ou leur famille: Elle n'écoute pas le côté de mon enfant. Elle le juge (12); L'enseignante n'est pas toujours objective envers mon fils (15); Elle m'a écrit une lettre comme quoi mon fils avait des très graves maux d'âme: "Il a des maux d'âme, il devrait être suivi par un psychiatre. [...] La solution finale, c'est le psychiatre. Il faut mettre de côté votre travail, puis faut laisser un expert prendre le dessus». En tout cas, j'ai pas aimé ça du tout de me faire dire ça! (90).

Le quart des parents reprochent au personnel enseignant d'utiliser de mauvaises manières de faire ou d'être avec leur enfant, soit lorsque l'enseignant fait preuve d'impatience, d'intolérance, d'insensibilité ou même de rejet: Lorsque l'enseignante est frustrée, elle se défoule sur mon enfant (47); L'enseignante crie toujours après lui (48); Il a eu une enseignante qui a carrément détruit son estime de soi (52); Elle a crié après mon fils dans le corridor (92); Il faut tout le temps qu'il l'abaisse (93); Je pense que mon fils ne se sent pas aimé. Il 
ne se sent pas valorisé. Puis, ça paraît. Ça paraît dans beaucoup de choses. Dans ses travaux à l'école, ça paraît (99); Des fois, elle repousse mon gars, parce qu'il est beaucoup, disons, actif. Elle le rejette «ben net» [complètement] (102); Je pense qu'elle lui aime pas la "fraise» [face] (109).

Deux autres parents se font critiques à l'endroit des jugements hâtifs que porte le personnel enseignant: Ça lui est arrivé de dire que mon enfant n'avait pas de "lunch» alors qu'il était dans son sac d'école (51).

Finalement, une mère trouve que l'enseignante n'accorde pas suffisamment le droit à l'erreur à son enfant: L'enseignante demande trop la perfection. C'est quand même un enfant, il faut le laisser respirer un peu (86). La nature des perceptions parentales négatives à l'endroit du personnel enseignant est présentée au tableau 4.31.

TAbleau 4.31

\section{La nature des perceptions parentales négatives à l'endroit du personnel enseignant $(n=31)$}

\begin{tabular}{lcccc}
\hline & \multicolumn{2}{c}{ Mères } & \multicolumn{2}{c}{ Pères } \\
\cline { 2 - 5 } & $\boldsymbol{N}$ & $\%$ & $\boldsymbol{N}$ & $\%$ \\
\cline { 2 - 5 } & 29 & 93,5 & 2 & 6,5 \\
\hline $\begin{array}{l}\text { A une gestion de classe déficiente } \\
\text { ou inappropriée }\end{array}$ & 19 & 61,5 & 1 & 3,0 \\
\hline $\begin{array}{l}\text { Manque de flexibilité } \\
\text { et de souplesse }\end{array}$ & 11 & 35,5 & 1 & 3,0 \\
\hline Manque de compréhension & 10 & 32,0 & & \\
\hline $\begin{array}{l}\text { A de mauvaises manières } \\
\text { de faire ou d'être }\end{array}$ & 8 & 26,0 & & \\
\hline Porte des jugements hâtifs & 2 & 6,5 & & \\
\hline $\begin{array}{l}\text { N'accorde pas suffisamment } \\
\text { le droit à l'erreur }\end{array}$ & 1 & 3,0 & & \\
\hline
\end{tabular}

\subsubsection{La direction d'école}

Chez près de la moitié des parents de ce sous-échantillon, les perceptions négatives concernent l'inaction de la direction d'école ou des actions qu' elle pose et qu'ils jugent inappropriées: Elle n'a pas fait grand-chose (09); La direction avait convoqué une rencontre avec sept personnes autour de la table. Ça, c'est des choses, pour nous autres, qui ne sont pas acceptables (14.1); Je n'aime pas la façon de faire du directeur. Il ne s'occupe pas trop des enfants qui ont des problèmes. Il n'a pas une bonne méthode. C'est pour ça que j'ai sorti mon fils de l'école, il n'avait pas assez d'aide. Je trouve qu'il faisait juste poser des questions. 
Il ne faisait rien d'autre pour lui. Il n'avait pas assez de services. L'enfant voyait une travailleuse sociale une fois par mois. C'est bon à rien puisque c'est une affaire urgente, parce que ça grandit. J'aimais pas sa manière de faire (24); Le directeur s'en lavait les mains. Il n'était pas vraiment intéressé à nous aider. Mais aussitôt qu'on lui a écrit une lettre pour lui expliquer nos insatisfactions, c'est là que ç'a changé un peu. Le directeur nous est revenu pour nous dire qu'il avait fait une demande pour avoir un psychologue pour notre fils (97).

Selon un peu plus du tiers des parents de ce sous-échantillon, la direction d'école les juge un peu trop sévèrement, tout en les tenant responsables de la situation-problème de leur enfant: En étant une mère seule, puis parce que j'ai 25 ans, je me fais traiter très mal de ce côté-là. J'ai pourtant une éducation collégiale même si j'ai eu mon enfant à 18 ans. Je trouve que c'est encore plus difficile à cause que je suis une mère seule (05); C'était une erreur de lui raconter ma situation familiale, parce qu'il a classé mon enfant. On s'attendait à ce que mon enfant agisse comme ça à cause de la séparation (11.1); Comme parents, on se fait traiter comme si on est le problème à cause de l'enfant (38).

L'âge de la mère à la naissance de l'enfant concerné ou du premier enfant, son niveau de scolarité, son statut marital, son groupe ethnoculturel, l'absence du père quelle qu'en soit la cause, ne sont que quelques-uns des motifs sur lesquels la direction d'école base son jugement. À ce sujet, il importe de rappeler que neuf mères de l'échantillon ont eu leur premier enfant avant l'âge de 20 ans. De ce nombre, huit familles sont monoparentales et vivent dans un contexte de pauvreté: Je suis monoparentale. Ça fait une grande différence (13); Si j'étais mariée, c'est pas comme ça qu'ils me parleraient. Parce que j'étais jeune, que j'étais une mère seule et aussi parce que je suis noire, je pense qu'ils n'avaient pas beaucoup de respect pour moi. Ça se voyait (112).

Un autre exemple de jugement porté à l'endroit d'un parent concerne le cas d'un enfant qui a été forcé de revenir à la maison en culotte courte peu de temps avant Noël, en raison du vol de son pantalon neuf. En un mois, la mère a effectué une quinzaine d'appels téléphoniques à la direction d'école pour expliquer et régler la situation. Alors qu' elle envisageait d'acheter une paire d'espadrilles à son enfant, elle reçoit un appel de l'enseignante au sujet du mauvais état de celles-ci. Malgré les démarches entreprises pour montrer qu'elle s'occupait bien de son fils, cette mère a été amenée à croire que le personnel scolaire la considérait négligente: Ça me faisait comme petite, petite, petite qu'ils pensaient que j'étais pas une bonne mère, que j'achetais pas d'affaires à mon fils (94).

Afin de contrer l'effet négatif de tels jugements, certains parents font preuve de réticences à transmettre des renseignements concernant leur situation familiale, estimant préférable d'établir une frontière claire avec 
le milieu scolaire: Ce n'est pas important pour moi que l'école sache tout de ma situation familiale. Il y a des choses qui ne les regardent pas (15).

D'après un peu moins du tiers des parents de ce sous-échantillon, la direction d'école manque de compréhension et d'écoute à l'endroit de leur enfant ou de leur famille: Oui, le directeur connaît ma situation familiale, mais il ne s'en préoccupe pas tellement (13); À la maison, je me suis toujours assise pour écouter mon enfant quand ça ne va pas. On dirait qu'il est écœuré de la vie. Il dit qu'à l'école, il n'y a personne qui l'écoute. Il a fallu qu'il fasse une crise cette année pour qu'on l'écoute (21); La directrice n'écoute pas mon enfant (32); J'ai pris le temps d'aller voir la direction pour lui parler des problèmes de mon enfant parce qu'il est hyperactif. Je lui ai dit mes inquiétudes par rapport à ça. Je me sentais frustrée à cause de son manque d'intérêt par rapport au problème de mon enfant. Je voulais éviter d'avoir des problèmes, puisque sa condition a un effet négatif sur l'estime de soi de mon enfant (38).

Également, un parent sur cinq reproche à la direction d'école de ne pas effectuer de suivi après une rencontre: Je pensais que l'école allait $m^{\prime} a p$ peler: "Y a-t-il quelque chose qui a changé depuis la rencontre? Non, rien.» Ça me frustre. On a tous nos moments dans la vie. On passe à travers des choses. Je téléphone, moi, pour dire ce qui en est (05). La direction d'école juge mon enfant trop vite. Je suis déçue. Je me bats pour lui. J'approche le directeur, mais il n'est pas fiable. Il n'est pas au rendez-vous pour mieux aider mon enfant. Il le blâme et ne gère pas adéquatement les situations. Il ne m'offre aucun support. Les problèmes de violence ne se sont pas arrangés suite à nos rencontres. On s'occupe plus des petites incidences (11). La nature des perceptions parentales négatives à l'endroit de la direction d'école est présentée au tableau 4.32.

TABLEAU 4.32

\section{La nature des perceptions parentales négatives à l'endroit de la direction d'école $(n=31)$}

\begin{tabular}{lcccc}
\hline & \multicolumn{2}{c}{ Mères } & \multicolumn{2}{c}{ Pères } \\
\cline { 2 - 5 } & $N$ & $\%$ & $N$ & $\%$ \\
\cline { 2 - 5 } & 29 & 93,5 & 2 & 6,5 \\
\hline $\begin{array}{l}\text { Est inactive ou pose des actions } \\
\text { inappropriées }\end{array}$ & 14 & 45,0 & 1 & 3,0 \\
\hline $\begin{array}{l}\text { Pose des jugements trop sévères } \\
\text { et entretient des préjugés }\end{array}$ & 10 & 33,5 & 1 & 3,0 \\
\hline $\begin{array}{l}\text { Manque de compréhension } \\
\text { et d'écoute }\end{array}$ & 9 & 29,0 & & \\
\hline $\begin{array}{l}\text { N'assure pas de suivi après } \\
\text { les rencontres }\end{array}$ & 6 & 19,5 & & \\
\hline
\end{tabular}




\subsubsection{Le personnel non enseignant}

Quatre parents de ce sous-échantillon entretiennent des perceptions négatives à l'endroit des membres du personnel non enseignant, soit plus précisément envers le personnel de soutien. Ces perceptions concernent des cas où des membres font preuve d'abus verbaux ou physiques à l'endroit de leur enfant: On a vu un très gros changement dans son comportement après que le conducteur l'ait secoué plusieurs fois dans l'autobus. Là, il devenait choqué, écœuré. Il disait qu'il allait le tuer. Comme il est bon pour nous conter des histoires, c'est pour ça que ça a pris du temps avant qu'on réalise ce qui se passait. C'est arrivé un soir qu'il a fait une crise dans l'autobus. Là, mon père a vu le conducteur en train de le secouer. Quand j'ai su ça, j'ai quitté mon travail et suis allée directement au bureau des autobus. Je leur ai dit que c'était la dernière fois que le conducteur d'autobus mettait la main sur mon enfant. Je leur ai dit que si ça se reproduisait, que j'allais l'emmener en cour. Environ un mois et demi plus tard, c'est encore arrivé. C'est là que j'ai explosé. J'ai déposé une plainte policière contre le conducteur d'autobus. Il y a eu une enquête. C'est la seule façon que j'ai eue d'obtenir un autre autobus pour mon fils. Maintenant, il est dans un petit autobus. Il est le dernier ramassé et le premier débarqué. Ce stress-là lui a été enlevé. On dirait que l'école ne comprend pas comment une petite chose peut tout changer. Dans son cas à lui, ça a été de changer de conducteur d'autobus (21).

\subsection{D'AUTRES PERCEPTIONS PARENTALES NÉGATIVES À L'ENDROIT DU MILIEU SCOLAIRE}

Parmi les perceptions négatives qu'entretiennent les parents, les trois quarts concernent le personnel scolaire en général et plus de la moitié se rapportent à l'école en général. Les autres perceptions parentales négatives à l'endroit du milieu scolaire sont présentées au tableau 4.33.

TABLEAU 4.33

\section{D'autres perceptions parentales négatives à l'endroit du milieu scolaire $(n=31)$}

\begin{tabular}{lcccc}
\hline & \multicolumn{2}{c}{ Mères } & \multicolumn{3}{c}{ Pères } \\
\cline { 2 - 5 } & $N$ & $\%$ & $N$ & $\%$ \\
\cline { 2 - 5 } & 29 & 93,5 & 2 & 6,5 \\
\hline Le personnel scolaire en général & 22 & 71,0 & 2 & 6,5 \\
\hline L'école en général & 20 & 54,5 & 1 & 3,0 \\
\hline
\end{tabular}




\subsubsection{Le personnel scolaire en général}

Parmi les perceptions négatives entretenues par les parents, les trois quarts se rapportent au personnel scolaire en général. La majorité de ces perceptions concernent le manque de temps pour s'occuper adéquatement de leur enfant: Il manque de surveillance sur l'heure du dîner, c'est épouvantable (11.1); À 30 élèves, ils n'ont pas d'aide lorsqu'il y a des situations (24); Je comprends que le personnel n'a pas toujours le temps de l'aider, mais ils font leur possible. Au moins, ils travaillent avec lui (58); L'école n'a pas beaucoup de temps à consacrer aux élèves de façon individuelle (80); L'école a trop d'élèves à s'occuper. Ce n'est pas leur problème, mais ils ne font pas grand-chose. Ils sautent tous au drame (88); C'est souvent, souvent quelque chose que tu entends: "On n'a pas le temps.»C'est souvent la réponse: "Il y a trop de coupures, on n'a pas le temps» (111).

Également, les deux tiers des parents de ce sous-échantillon n'apprécient pas la tendance qu'ont certains membres du personnel scolaire à blâmer leur enfant: On la blâme toujours à cause de son passé (12); Il se fait blâmer souvent (80); L'école est portée à le blâmer parce que c'est un enfant difficile. Si un autre enfant dit que mon fils a fait telle chose, on croit tout de suite que c'est lui (85).

Devant un tel blâme, certains parents ont tendance à leur tour à rejeter le blâme sur le personnel scolaire: J'avais essayé de parler à la directrice et au professeur, d'utiliser des méthodes qui préviennent les situations à risque. [...] Je trouve que ça fait une grosse différence. Puis, justement, à ce moment-là, je les blâmais (04).

$\mathrm{Au}$ lieu de blâmer leur enfant, ces parents souhaiteraient que les membres du personnel scolaire s'adaptent davantage aux besoins de leur enfant: Mais quand même, d'essayer de s'adapter aux besoins de l'enfant, pas seulement appliquer des techniques (04); Il faut être attentif à l'enfant. Ça aide l'enfant quand il voit que tu es attentif à lui, au lieu d'être choqué après lui, puis de le blâmer pour ça (24).

Ces mêmes parents vont encore plus loin en exprimant le souhait que le personnel scolaire fasse davantage preuve de chaleur humaine envers l'enfant: Je me dis que lorsque l'enfant fait quelque chose de méchant, il ne faut pas juste regarder la chose, mais son cri du cœur et son appel à l'aide (04); Au lieu de le chicaner, il faut essayer d'avoir un contact humain. C'est important, ça. Il a besoin d'amour, il faut lui montrer qu'on l'aime (24).

Par ailleurs, la moitié des parents reprochent au personnel scolaire son incapacité à reconnaître les qualités personnelles de leur enfant à leur juste valeur: C'est vraiment un bon garçon, mon gars, même si c'est quelqu'un qui prend beaucoup de place. Ça, l'école est pas capable de le reconnaître! (11.1); 
C'est un enfant joyeux et affectueux. C'est vrai qu'il bouge beaucoup, mais c'est parce qu'il a besoin de contacts physiques. Pourquoi l'école ne comprend-elle pas ça? (48); L'école dit que ma fille est violente, mais elle l'est pas. Je sais que c'est pas un ange, mais au moins ça je peux l'admettre (107); Je sais que ma fille aime beaucoup l'attention, mais elle est vraiment intelligente. Jamais l'école ne m'a parlé de ça (112). La nature des perceptions parentales négatives à l'endroit du personnel scolaire en général est présentée au tableau 4.34.

TABLEAU 4.34

\section{La nature des perceptions parentales négatives à l'endroit du personnel scolaire en général $(n=31)$}

\begin{tabular}{lcccc}
\hline & \multicolumn{2}{c}{ Mères } & \multicolumn{2}{c}{ Pères } \\
\cline { 2 - 5 } & $N$ & $\%$ & $N$ & $\%$ \\
\cline { 2 - 5 } & 29 & 93,5 & 2 & 6,5 \\
\hline Manque de temps & 22 & 71,0 & 2 & 6,5 \\
\hline A tendance à blâmer l'enfant & 20 & 64,5 & 1 & 3,0 \\
\hline $\begin{array}{l}\text { Se fait à son tour rejeter } \\
\text { le blâme par les parents }\end{array}$ & 17 & 59,0 & 1 & 3,0 \\
\hline $\begin{array}{l}\text { Est incapable de reconnaître } \\
\text { les qualités de l'enfant }\end{array}$ & 16 & 51,5 & & \\
\hline $\begin{array}{l}\text { Devrait s'adapter davantage } \\
\text { aux besoins de l'enfant }\end{array}$ & 4 & 13,0 & & \\
\hline $\begin{array}{l}\text { Devrait faire davantage preuve } \\
\text { de chaleur humaine }\end{array}$ & 2 & 6,5 & & \\
\hline
\end{tabular}

\subsubsection{L'école en général}

Parmi les parents qui entretiennent des perceptions négatives, la moitié a trait à un manque de ressources de l'école en général. À ce sujet, les parents notent qu'un manque de ressources financières nuit au processus de résolution de la situation-problème de leur enfant: L'école ne nous a pas offert d'aide. Il a fallu que nous en demandions. Je pense que c'est à eux de faire le boulot. À cause du manque de budget, ils ne le font pas (02).

Selon deux parents sur cinq de ce sous-échantillon, plus de ressources financières signifient davantage de possibilités d'offrir des services afin de répondre adéquatement à la demande lorsqu'elle est formulée: Il faudrait offrir plus de services à l'école (09); Je demande des services pour mon fils et je ne les reçois pas (74). 
Par ailleurs, le tiers des parents notent que le manque de ressources humaines spécialisées représente un élément affectant beaucoup la situation-problème de leur enfant: Juste avoir une éducatrice, c'est très difficile dans le système actuel (20); L'école n'a pas de personnel suffisant pour régler le problème (45).

De plus, l'accès à de telles ressources humaines spécialisées lorsqu'elles sont disponibles nécessite de longs mois d'attente: Je trouve dommage que l'école ait pris autant de temps avant de donner des services à mon enfant. Il a besoin d'une approche différente. Il lui faut une aide individuelle. Cela l'aide à fonctionner. Il en a plus besoin que les autres. C'est seulement à la fin de l'année scolaire qu'il a pu en obtenir (82); C'était au moins six mois d'attente. Mais là, il est sur une liste prioritaire (90).

Les interventions et les suivis que les parents entreprennent en sont également affectés: J'ai eu une rencontre avec le centre, mais ils ne m'ont pas encore rappelée. Je n'ai pas assez d'argent pour aller chercher de l'aide externe (82); Il y a eu une évaluation qui a été faite, c'est tout. Il n'y a pas eu de suivi après ça. Il y a eu des recommandations générales de la psychologue, mais pas de réunion comme telle pour dire "O.K., c'est ce qu'on fait» (111). La nature des perceptions parentales négatives à l'endroit de l'école en général est présentée au tableau 4.35 .

TABleau 4.35

La nature des perceptions parentales négatives à l'endroit de l'école en général $(n=31)$

\begin{tabular}{lcccc}
\hline & \multicolumn{2}{c}{ Mères } & \multicolumn{2}{c}{ Pères } \\
\cline { 2 - 5 } & $N$ & $\%$ & $N$ & $\%$ \\
\cline { 2 - 5 } & 29 & 93,5 & 2 & 6,5 \\
\hline Manque de ressources financières & 15 & 48,5 & 1 & 3,0 \\
\hline Manque de services & 12 & 39,0 & 1 & 3,0 \\
\hline $\begin{array}{l}\text { Manque de ressources humaines } \\
\text { spécialisées }\end{array}$ & 10 & 32,0 & 1 & 3,0 \\
\hline $\begin{array}{l}\text { Nécessite une trop longue } \\
\text { période d'attente }\end{array}$ & 8 & 26,0 & & \\
\hline $\begin{array}{l}\text { Manque d'interventions et } \\
\text { de suivis }\end{array}$ & 6 & 19,5 & & \\
\hline
\end{tabular}




\subsection{EN RÉSUMÉ}

Trois parents sur quatre de l'échantillon entretiennent des perceptions positives à l'endroit du personnel scolaire, alors que la moitié d'entre eux entretiennent des perceptions négatives. La moitié des parents perçoivent le personnel enseignant $d^{\prime}$ une manière favorable. Ils apprécient notamment l'attention personnalisée accordée à leur enfant. À l'inverse, un parent sur trois entretient des perceptions négatives à l'endroit du personnel enseignant. La direction d'école apparaît également comme une personneclé pour garantir l'accès à des ressources humaines spécialisées et à des services pouvant être mis à la disposition de l'enfant afin de lui venir en aide. L'ensemble de ces perceptions jettent un éclairage sur les facteurs de soutien ou les obstacles sous-jacents à la collaboration entre l'école et les parents.

Le blâme mutuel ainsi que le transfert de responsabilités d'un milieu à l'autre, concernant la situation-problème de l'enfant, semblent être également un élément important de tensions entre l'école et les parents. La nature des échanges contribue de nouveau à mieux saisir en quoi l'établissement de frontières claires entre les deux milieux peut soit contribuer à la résolution de la situation-problème de l'enfant, soit l'alimenter. À cet égard, le personnel enseignant peut se sentir jugé et ses pratiques pédagogiques remises en question. C'est pourquoi, afin de ne pas être menacé dans son identité et son statut professionnel, il maintient un tel fossé avec les parents (Cifali, 1997; Montandon, 1994).

Quant au parent, il a peur d'être pointé du doigt par le personnel enseignant lors de difficultés (Dubet et Martuccelli, 1996). De telles réticences s'appliquent également à la direction d'école, qui est davantage associée à l'institution, à ses procédures, au pouvoir décisionnel, dans ce que Montandon et Perrenoud (1994) appellent l'asymétrie de pouvoirs entre les milieux scolaire et familial.

Également, pour deux parents sur cinq, le manque de ressources financières et de temps exacerbe une situation-problème déjà porteuse de tensions. À l'asymétrie de pouvoirs s'ajoute un obstacle de taille, soit l'accès à des ressources humaines spécialisées et des services pouvant être mis à la disposition tant de l'enfant que des parents afin de leur venir en aide.

Bref, l'analyse des résultats sur la collaboration entre l'école et les parents appelle à un véritable départage des responsabilités entre les individus et les institutions. Ces résultats incitent à poursuivre l'analyse des contextes difficiles, parsemés de tensions et d'embûches. Tous ces obstacles alimentent la situation-problème de l'enfant, en générant un 
potentiel de conflits entre l'école et les parents. Il appartient à chacun des milieux scolaire et familial de les dépasser afin d'être des acteurs soutenant la collaboration.

\section{LES GESTES D'EMPOWERMENT POSÉS PAR LES PARENTS}

Dans l'idée que les milieux scolaire et familial doivent surmonter des obstacles afin de devenir des partenaires à part entière qui soutiennent la collaboration, nous accordons une attention particulière aux gestes d'empowerment posés par les parents. Ces gestes leur permettent d'exercer une meilleure influence, voire un contrôle sur la situation-problème de leur enfant à l'école.

Les parents peuvent poser trois types de gestes d'empowerment: des gestes d'adaptation, d'opposition et de résistance. Les gestes d'adaptation sont les actions qui visent à maîtriser, à tolérer et à réduire l'impact négatif d'un événement, d'une décision, etc. (Desmarais, Beauregard, Guérette, Hrimech, Lebel, Martineau et Péloquin, 2000). Quant aux gestes d'opposition, ils visent à contrecarrer les décisions déjà prises par l'école, ainsi qu'à mobiliser des ressources pour influencer positivement et améliorer la situation-problème de l'enfant. Enfin, les gestes de résistance sont des actes intentionnels ou des omissions volontaires de la part des parents qui défient les désirs de membres du personnel scolaire (Ashforth et Mael, 1998). Il peut également s'agir de pratiques silencieuses de protestation.

\subsection{LES GESTES D'ADAPTATION, D'OPPOSITION ET DE RÉSISTANCE}

Près de quatre parents sur cinq de l'échantillon posent au moins un geste d'empowerment. Sur ce nombre, 12 parents sont plus actifs que les autres. Parmi eux, neuf ont des enfants de 6 ans et moins. Cinq des 12 parents ont posé plus de six gestes différents d'adaptation, d'opposition ou de résistance. Ils correspondent tous au profil de non-collaboration. Parfois, nous détectons également de l'agressivité dans leurs propos. Ces parents possèdent tous une formation professionnelle spécialisée. Quant aux sept autres parents actifs, ils ont posé cinq gestes différents d'adaptation, d'opposition ou de résistance. Quatre de ces sept parents ont une formation universitaire. 


\subsection{L'ADAPTATION POUR UNE RÉSOLUTION DE LA SITUATION-PROBLÈME}

Parmi les parents qui posent des gestes d'adaptation, deux sur cinq amorcent un processus de résolution de la situation-problème. Afin $d^{\prime} y$ parvenir, le tiers d'entre eux convoquent des rencontres avec le personnel scolaire dans le but de proposer des solutions: Il a fallu que je rassemble cing personnes de l'école pour leur parler de la façon dont j'interviens avec mon fils (85).

$\mathrm{Au}$ sujet de ces rencontres, les parents témoignent de leur perception d'agir sur les choses et les événements, tout en s'appropriant une forme de pouvoir. Ce sentiment d'exercer une influence ou de contrôler la situation-problème est corroboré par des résultats se révélant parfois positifs à la suite de leur action: J'avais suggéré au directeur qu'il demande à l'enseignante qu'après l'école, elle me montre les devoirs à faire. Ils ont collaboré. C'était positif (24).

Les solutions proposées par le tiers des parents varient grandement de l'un à l'autre: isolement de l'enfant, davantage d'attention, repos supplémentaire, retrait de privilèges, davantage de responsabilités, technique 1-2-3, mentorat et renforcement positif: S'il n'écoute pas du tout, il faut l'envoyer au bureau ou m'appeler, un des deux (102).

Les parents proposent d'autres solutions, telles qu'un programme éducatif pour la maison, de l'aide dans la classe, la structuration de petits groupes de récupération, la réduction du nombre de devoirs et l'ajout de tâches ou de matériel pour tenir l'enfant occupé: «Donne-lui quelque chose d'autre à faire après l'école. Laisse-le 10-15 minutes de plus à l'école». Ils ont fini par m'écouter. C'est finalement ce qu'ils ont fait (94).

Les prises de contact avec le personnel scolaire poursuivent divers autres buts. À cet effet, près du tiers des parents désirent discuter d'événements relatifs aux comportements violents de leur enfant ou assurer un suivi suite à ces événements: L'enseignante m'a envoyée un peu dans les fleurs et c'est là qu'on a eu une rencontre avec elle et la directrice. J'avais écrit une lettre à la directrice pour lui expliquer ce qui s'était passé à mon arrivée à l'école (01).

Pour sept parents, les échanges avec le personnel scolaire visent à savoir comment aider son enfant ou à mieux comprendre le quotidien vécu au préscolaire et au primaire. Or, ce même geste favorable à une collaboration peut être empreint d'agressivité de la part du parent, s'il s'inscrit dans une relation conflictuelle: "Je veux des rapports, je veux savoir exactement ce que vous avez essayé. J'attends un rapport complet de chacun de ses agissements» (109). 
Pour six autres, de tels échanges servent à introduire leur enfant à l'école: Quand il a commencé l'école, j'ai dit: «Écoutez, mon enfant, il est, disons, difficile. S'il est bien fâché, il devient agressif. Ça peut lui arriver de lancer des choses» (108).

Ce type de contact peut être établi au début de chaque année scolaire: Je vais toujours rencontrer la maîtresse pour expliquer un petit peu ce qu'il a vécu. Cette année, j'ai même donné la fiche de comportement qu'il avait l'année passée (90).

Lors de ces échanges, les parents peuvent en profiter pour adresser quelques mises en garde qui sont parfois mal interprétées par certains membres du personnel scolaire: Je suis allée voir le directeur pour lui dire que je ne veux pas être mis au courant à la dernière minute ou lorsque ça arrive la troisième fois. Je veux le savoir la première fois! (83).

Ces discussions, lorsqu'elles se situent dans un contexte de collaboration, permettent toutefois d'arriver à des solutions satisfaisantes: J'ai été voir le directeur le lendemain. J'ai dit: "Je m'excuse». La maîtresse, elle, s'est excusée à mon fils. Puis ça s'est bien arrangé (06).

Par ailleurs, à l'instar de l'analyse portant sur les modes de communication entre l'école et les parents vivant en contexte de pauvreté, les gestes $\mathrm{d}^{\prime}$ empowerment de ces derniers revêtent parfois un caractère plus direct: Je ne demandais même pas à le rencontrer, j'allais tout de suite à son bureau (92).

Or, la fréquence des contacts est généralement associée aux difficultés encourues par l'enfant (Adams et Christenson, 2000). Recevoir de nombreux appels téléphoniques et se rendre souvent à l'école pour discuter de la situation-problème de l'enfant risque surtout d'engendrer des mésententes: Elle était fâchée contre moi, bla, bla, bla. Le lendemain, je suis rentrée chez la directrice (108). Au fur et à mesure que la situation dégénère, d'autres acteurs sont impliqués: Mon mari a appris à s'exprimer concernant ses enfants. Ça lui est arrivé de décrocher le téléphone pour dire: «Écoutez, moi, je veux avoir plus de communication sur ce qui se passe à l'école» (99).

Finalement, le dernier geste d'adaptation posé par le tiers des parents de ce sous-échantillon est de consulter un tiers à l'extérieur à l'école, soit un médecin, un psychologue privé ou une intervenante communautaire, dans le cas des familles vivant en contexte de pauvreté. Ces parents s'informent pour mieux connaître la condition de leur enfant et pouvoir l'améliorer. Par la suite, ils utilisent ces avis pour faciliter leurs négociations avec le personnel scolaire ou pour contrecarrer l'expertise des professionnels œuvrant en milieu scolaire: "Je cherche un psychologue parce que je veux le faire évaluer par quelqu'un de plus compétent.» Puis, je sortais des documents et je lui disais: "Madame, ces documents ont été écrits par des psychologues et 
regardez, ils ne sont pas d'accord avec vous » (11.1); J'ai devancé l'école en faisant investiguer les yeux et les oreilles de mon fils (48); Je vais en parler avec mon médecin, puis je vais passer des évaluations de comportement avec lui (102).

Sur le nombre de parents qui ont recours à un tiers, neuf consultent un psychologue privé et une famille bénéficie d'un tel service par le biais d'un centre d'aide à la famille. Pour cinq d'entre eux, le psychologue intervient directement dans le milieu scolaire: J'ai fait venir le psychologue à l'école pour qu'il parle à la directrice et au professeur (01); Avec eux autres, il fallait toujours qu'elle vienne avec moi, parce que je sentais qu'ils me respectaient un peu plus quand elle était là (112). Les gestes d'adaptation posés par les parents sont présentés au tableau 4.36.

TABLEAU 4.36

Les gestes d'adaptation posés par les parents $(n=48)$

\begin{tabular}{lcccc}
\hline & \multicolumn{2}{c}{ Mères } & \multicolumn{2}{c}{ Pères } \\
\cline { 2 - 5 } & $N$ & $\%$ & 4 & $\%$ \\
\cline { 2 - 5 } & 44 & 91,5 & 1 & 2,0 \\
\hline $\begin{array}{l}\text { Amorcent la résolution de la } \\
\text { situation-problème }\end{array}$ & 20 & 41,5 & 2 & 4,0 \\
\hline $\begin{array}{l}\text { Convoquent une rencontre avec } \\
\text { le personnel scolaire }\end{array}$ & 17 & 35,5 & 1 & 2,0 \\
\hline Proposent des solutions & 15 & 31,5 & 1 & 2,0 \\
\hline $\begin{array}{l}\text { Consultent un tiers extérieur } \\
\text { à l'école }\end{array}$ & 14 & 29,0 & 1 & 2,0 \\
\hline $\begin{array}{l}\text { Discutent des comportements } \\
\text { ou assurent un suivi }\end{array}$ & 13 & 27,0 & & \\
\hline $\begin{array}{l}\text { Échangent sur la manière d'aider } \\
\text { et sur le quotidien }\end{array}$ & 7 & 14,5 & & \\
\hline \begin{tabular}{l} 
Introduisent l'enfant à l'école \\
\hline
\end{tabular} & 6 & 12,5 & & \\
\hline
\end{tabular}

\subsection{L'OPPOSITION EN VUE D'UNE MOBILISATION DES RESSOURCES}

Pour deux parents sur cinq de ce sous-échantillon, l'opposition est un moyen d'exercer une influence sur les décisions déjà prises par l'école: J'étais vraiment pas d'accord avec la directrice et on s'est «chicané» [engueulé] (32).

Ces parents s'affirment également dans le but d'obtenir des services pouvant être mis à la disposition de leur enfant afin de leur venir en aide. Lorsque les parents décident de s'opposer aux décisions déjà prises par 
l'école, le ton peut monter d'un cran: Je leur ai dit que jamais plus je me plierai à leur demande. Jamais! (85).

En relation avec de tels échanges conflictuels, les parents peuvent ressentir une importante source d'agressivité et de stress découlant de leur insatisfaction: Je me suis chicanée souvent avec le directeur adjoint. Je lui ai dit que s'il ne cessait pas de me harceler, que j'allais appeler mon avocat [...]. Je me suis retenue souvent de ne pas dire des gros mots au directeur adjoint, pour ne pas mettre de l'huile sur le feu et pour ne pas que l'enfant paye (48).

Treize des 19 parents qui s'opposent aux interventions majeures de l'école font partie du profil de non-collaboration. Leurs relations avec les membres du personnel scolaire sont généralement tendues et empreintes d'émotivité: Je lui ai dit qu'il aurait intérêt à s'occuper du cas de mon fils. Mon amie m'a tapé sur l'épaule en voulant dire «Calme-toi» et j'ai dit: "Elle ne me laisse pas placer un mot!» (11.1).

Dans les cas les plus extrêmes, les négociations avec l'école se font sous le signe de la confrontation: J'ai levé le ton. Je leur ai dit ce que je pensais d'eux autres, qu'ils étaient une "gang» d'incompétents. Je les ai vraiment sur le cœur. Je leur ai dit que je pouvais les amener en cour (93).

Dans la lignée de cette opposition, les gestes d'empowerment posés par les parents sont diversifiés. Ceux-ci effectuent plusieurs actions concrètes en vue d'une mobilisation des ressources et admettent avoir beaucoup de demandes et d'attentes face à l'école. Ils souhaitent bénéficier davantage de suivi suite à leurs rencontres, afin d'exercer une meilleure influence, voire un contrôle sur la situation-problème de leur enfant à l'école. Pour sept parents, il s'agit de documenter les propos et les actions du personnel scolaire: J'avais une petite enregistreuse dans ma poche. Je voulais enregistrer ce qu'ils me disaient pour me protéger moi-même, pour protéger mon fils (93).

Quatre d'entre eux observent l'enfant à son insu à l'école: J'ai passé trois semaines, tous les jours, toutes mes heures de dîner, dans la cour d'école, caché en arrière d'un arbre pour les voir faire, savoir ce qui se passait, savoir si vraiment c'est tout le temps mon fils qui commençait (93).

Trois autres parents composent un dossier accompagné de copies des lettres expédiées, d'une liste des rencontres tenues, des problèmes vécus, des demandes faites et restées sans réponse. Ces dossiers peuvent servir de preuve, voire de caution, lorsque les parents effectuent des démarches à un autre palier administratif.

Quatre parents mentionnent avoir exercé de fortes pressions sur le conseil scolaire pour obtenir davantage de ressources humaines spécialisées et de services de la part de l'école: J'ai insisté pour avoir des services et des ressources. Je n'ai jamais lâché jusqu'à ce que je les obtienne (48); Le conseil scolaire ne retournait pas mes appels. J'ai été obligée d'écrire une lettre (84). 
Par ailleurs, devant toutes ces difficultés à exercer une influence, voire un contrôle sur la situation-problème, un parent sur cinq de ce souséchantillon pose des gestes plus radicaux. Ceux-ci changent leur enfant d'école, de garderie scolaire ou de classe: Je l'ai fait changer d'école. Puis, c'était la plus belle affaire que j'ai faite (92).

Trois d'entre eux privilégient l'enseignement à la maison: C'est arrivé deux fois. L'année passée, il est resté six mois à la maison parce que je l'ai sorti de l'école. C'est moi qui lui ai fait l'école à la maison (24).

Enfin, deux parents qui s'opposent au Ritalin ${ }^{\circledR}$ se résignent finalement à médicamenter leur enfant à la suite des fortes pressions exercées par les membres du personnel scolaire. Ils posent toutefois des gestes d'empowerment dans la mesure où ils contrôlent le dosage et la durée de la prise du médicament: C'est moi qui contrôle le dosage du Ritalin ${ }^{\circledR}$ à l'école (48); On est d'accord pour essayer le Ritalin ${ }^{\circledR}$ pour cinq ou six mois. Après ça, graduellement, on va l'enlever (93). Les gestes d'opposition posés par les parents sont présentés au tableau 4.37 .

TABLEAU 4.37

Les gestes d'opposition posés par les parents $(n=48)$

\begin{tabular}{|c|c|c|c|c|}
\hline & \multicolumn{2}{|c|}{ Mères } & \multicolumn{2}{|c|}{ Pères } \\
\hline & $N$ & $\%$ & $N$ & $\%$ \\
\hline & 44 & 91,5 & 4 & 8,5 \\
\hline $\begin{array}{l}\text { S'opposent aux interventions } \\
\text { majeures de l'école }\end{array}$ & 19 & 39,5 & 1 & 2,0 \\
\hline $\begin{array}{l}\text { Changent leur enfant d'école } \\
\text { ou de garderie }\end{array}$ & 11 & 23,0 & 2 & 4,0 \\
\hline $\begin{array}{l}\begin{array}{l}\text { Documentent les actions et les } \\
\text { propos du personnel scolaire }\end{array} \\
\end{array}$ & 5 & 10,5 & 2 & 4,0 \\
\hline $\begin{array}{l}\text { Exercent des pressions sur } \\
\text { le conseil scolaire }\end{array}$ & 4 & 8,5 & & \\
\hline $\begin{array}{l}\text { Insistent pour obtenir des } \\
\text { ressources et des services }\end{array}$ & 3 & 6,5 & 1 & 2,0 \\
\hline $\begin{array}{l}\text { Observent leur enfant à son insu } \\
\text { à l'école }\end{array}$ & 3 & 6,5 & 1 & 2,0 \\
\hline Composent un dossier formel & 3 & 6,5 & & \\
\hline $\begin{array}{l}\text { Enseignent à leur enfant } \\
\text { à la maison }\end{array}$ & 3 & 6,5 & & \\
\hline Changent leur enfant de classe & 2 & 4,0 & & \\
\hline $\begin{array}{l}\text { Contrôlent le dosage et la durée } \\
\text { du Ritalin }{ }^{\circledR}\end{array}$ & 2 & 4,0 & & \\
\hline
\end{tabular}




\subsection{LA RÉSISTANCE COMME AUTRE OPTION}

Les parents posent des gestes d'empowerment afin de surmonter les obstacles qu'ils perçoivent dans leurs échanges avec l'école. Si, par ces gestes, ils visent, dans l'ensemble à s'adapter à la situation-problème ou à s'opposer aux décisions déjà prises par l'école, certains d'entre eux font aussi preuve de résistance. Le recours à ce type de gestes vise à défier l'autorité du milieu scolaire. Il peut également s'agir de pratiques silencieuses de protestation, qui consistent soit à se conformer aux demandes émanant de l'école ou à les ignorer, soit même à adopter des comportements de fuite pour éviter de vivre des tensions trop importantes, voire d'entrer en conflit avec les membres du personnel scolaire.

Près du tiers des parents de ce sous-échantillon déclarent se conformer aux demandes émanant du personnel scolaire, tout en maintenant leur sentiment d'exercer une influence, voire un contrôle sur la situationproblème de leur enfant à l'école. Pour eux, se conformer constitue un geste d'empowerment (Pinderhughes, 1995). Selon une lecture stratégique de l'action, ils préfèrent se plier aux règles de l'institution pour acheter la paix: Je ne suis pas vraiment portée à chercher à influencer les autres. J'aime faire ce que j'ai à faire et je dis seulement ce que j'ai à dire (12.1); On a juste accepté où ils voulaient l'envoyer (44); Je m'oppose pas à ce qu'ils proposent (111).

Pour certains autres parents, se taire et adopter la position du personnel scolaire permettent de maintenir une bonne collaboration: On a tendance à ne pas en parler parce que ça retombe sur nous. On se fait critiquer (44); J'étais un peu fâchée, puis j'ai pas dit grand-chose, j'ai juste dit «O.K.» (112).

À l'inverse, s'opposer aux décisions déjà prises par l'école risque d'envenimer la situation-problème de l'enfant: On se serait juste engueulé... C'était une perte de temps. J'ai rempli les petits papiers qu'elle voulait (11.1).

Ce désir de conformisme génère toutefois une forme de désengagement chez certains d'entre eux: Ma fille va avoir un psychologue ou une travailleuse sociale. J'ai signé et j'ai dit: "O.K. Faites ce que vous voulez» (95); Ils m'ont demandé ce que j'en pensais. Mais, je ne voulais pas être contre eux, car mon enfant a un problème et c'est comme ça qu'ils règlent ça (98).

Par ailleurs, huit parents ignorent les demandes émanant de l'école: Si j'avais un problème, ça ne regarderait pas l'école et je ne dirais rien (12); On ne voulait plus participer après la première rencontre, ça ne marchait pas (20); Je paye pas pour les fameux poteaux (32); Inquiète-toi pas, j'ai un autre suçon dans ma poche (107). 
Finalement, deux parents adoptent des comportements de fuite: «Vite, viens-t'en, on s'en va chez nous» (91); J'étais plus capable d'en prendre. J'en avais assez entendu. J'ai pris la porte. Je m'en suis retourné travailler, c'était plus payant (93). Les gestes de résistance posés par les parents sont présentés au tableau 4.38 .

TABLEAU 4.38

Les gestes de résistance posés par les parents $(n=48)$

\begin{tabular}{lcccc}
\hline & \multicolumn{2}{c}{ Mères } & \multicolumn{2}{c}{ Pères } \\
\cline { 2 - 5 } & $N$ & $\%$ & $N$ & $\%$ \\
\cline { 2 - 5 } & 44 & 91,5 & 4 & 8,5 \\
\hline $\begin{array}{l}\text { Se conforment aux demandes } \\
\text { de l'école }\end{array}$ & 13 & 27,0 & 1 & 2,0 \\
\hline $\begin{array}{l}\text { Se taisent et adoptent la position } \\
\text { de l'école }\end{array}$ & 13 & 27,0 & 1 & 2,0 \\
\hline $\begin{array}{l}\text { Ignorent les demandes émanant } \\
\text { de l'école }\end{array}$ & 6 & 12,5 & 2 & 4,0 \\
\hline $\begin{array}{l}\text { Se désengagent de la } \\
\text { situation-problème }\end{array}$ & 2 & 4,0 & & \\
\hline $\begin{array}{l}\text { Adoptent des comportements } \\
\text { de fuite }\end{array}$ & 2 & 4,0 & & \\
\hline
\end{tabular}

\subsection{EN RÉSUMÉ}

Quatre parents sur cinq de l'échantillon posent au moins un geste d'empowerment, afin d'exercer une influence, voire un contrôle sur la situationproblème de leur enfant à l'école, que ce soit pour s'adapter, s'opposer ou résister. Concernant ces gestes, l'analyse des données d'entrevues ne révèle aucune distinction par rapport aux variables sociodémographiques relatives à l'enfant, aux parents ou à la famille.

Parmi les gestes d'adaptation posés par les parents, il peut s'agir d'amorcer un processus de résolution de la situation-problème, de convoquer une rencontre, de proposer des solutions, de consulter un tiers à l'extérieur à l'école, de prendre contact avec le personnel scolaire afin de discuter du comportement de l'enfant, d'assurer un suivi suite à ces comportements, d'introduire leur enfant, d'échanger sur la manière de l'aider ou sur sa réalité quotidienne vécue à l'école. 
Parmi les gestes d'opposition, des parents choisissent de contrecarrer les interventions majeures de l'école et de prendre des mesures plus radicales, telles que changer leur enfant d'école, de garderie scolaire ou de classe. Les parents peuvent également documenter les actions et les propos du personnel scolaire et observer l'enfant à son insu à l'école. Ces gestes permettent, dans certains cas, de constituer un dossier formel pouvant servir de preuve, voire de caution, à présenter à un autre palier administratif. Finalement, quelques-uns font des pressions auprès du conseil scolaire et insistent pour obtenir des ressources humaines spécialisées et des services, enseignent à l'enfant à la maison ou se résignent à le médicamenter au Ritalin ${ }^{\circledR}$.

Quant aux gestes de résistance, ils consistent à se conformer aux demandes de l'école, à se taire et à adopter la position du milieu scolaire. Il peut également s'agir d'ignorer les demandes émanant du personnel scolaire, de se désengager de la situation-problème ou même d'adopter des comportements de fuite pour éviter d'entrer en conflit avec le personnel scolaire.

Le geste d'empowerment qui semble toutefois le plus positif consiste à consulter un tiers extérieur à l'école. Selon la majorité des parents de l'échantillon, la consultation d'un tiers permet d'améliorer la situationproblème de l'enfant tout en permettant une collaboration avec l'école dans un esprit d'équipe et un respect mutuel.

Par ailleurs, les parents qui s'impliquent à l'école, soit en faisant partie de comités, soit dans des activités de bénévolat, ont davantage d'occasions de poser des gestes d'empowerment. L'influence, voire le contrôle qu'ils exercent à ces occasions sur la situation-problème de leur enfant à l'école ne peut toutefois pas être concrètement défini à partir de leur discours. Il n'est pas non plus possible d'identifier les gestes ou les actions concrètes qu'ils posent parce qu'ils ne se sont pas prononcés sur le sujet.

Quoi qu'il en soit, le fait de poser de nombreux gestes d'empowerment peut être le signe de tensions entre l'école et les parents. Ces gestes, tout comme la fréquence des contacts, sont généralement associés aux difficultés encourues par l'enfant (Kohl, Lengua et McMahon, 2000; Marcon, 1999; Reynolds, Weissberg et Kasprow, 1992). Par conséquent, ce n'est pas la quantité de gestes posés qui importe, mais le contexte dans lequel ils s'inscrivent. Lorsqu'un parent du profil de collaboration pose un geste d'empowerment, il a davantage de chances de contribuer à la résolution de la situation-problème de son enfant. À l'inverse, le même geste posé par un parent du profil de non-collaboration peut dégénérer en tensions, voire en une relation conflictuelle avec l'école. 
Il n'en demeure pas moins que les parents de l'échantillon sont des acteurs qui agissent dans le but de contribuer à l'amélioration et au mieux-être de leur enfant. À leurs yeux, lorsqu'ils obtiennent des gains, il s'agit d'une réelle collaboration avec l'école. Ces gains prennent une grande importance lorsque les parents réussissent non seulement à se faire entendre, mais également à prendre des décisions communes avec le personnel scolaire, notamment sur la question d'un suivi professionnel, d'un placement dans une école ou une classe spéciale, de la prise de médicament ou d'une suspension scolaire.

Dans le cas de la suspension scolaire, les parents sont toutefois particulièrement préoccupés par les conséquences et les bouleversements qu'elle occasionne dans la vie de leur enfant ainsi que dans leur propre vie; c'est pourquoi le prochain chapitre y est entièrement consacré. 


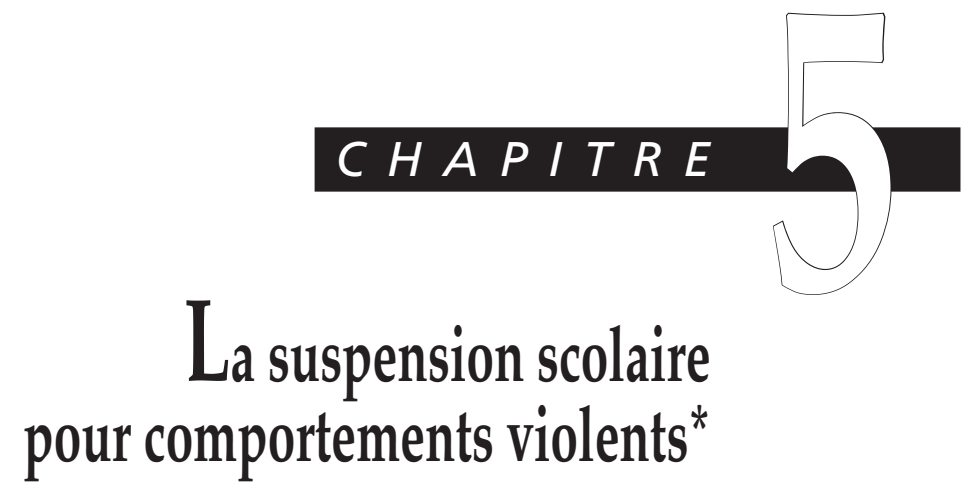

* Des extraits de ce chapitre sont tirés de: M. Paquin et M. Drolet (2004). "Comportements violents chez l'enfant en Ontario: problématique de la suspension scolaire externe, perceptions des parents et alternative possible», Éducation et francophonie, numéro spécial sur la violence à l'école, 32(1), p. 201-233. 

Le cinquième chapitre de cet ouvrage porte sur la suspension scolaire, un enjeu crucial lié à la problématique des enfants ayant des comportements violents envers leurs pairs au préscolaire et au primaire. Nous y présentons les perceptions des parents d'enfants de 3 à 9 ans faisant l'objet d'une suspension scolaire ou d'une possibilité de suspension, en réponse à de tels comportements. Les résultats sont présentés globalement quoique la suspension scolaire soit davantage appliquée chez les 6 à 9 ans que chez les 3 à 6 ans. Ces derniers sont plutôt aux prises avec une possibilité d'application de la mesure disciplinaire. Nous exposons la problématique de la suspension scolaire en traçant l'origine de la mesure disciplinaire, ses avantages et inconvénients, les perceptions que les parents de l'échantillon entretiennent face à son application ainsi qu'une alternative pouvant possiblement être utilisée afin de ne pas entraver le processus de scolarisation et de socialisation de leur enfant.

\section{LA PROBLÉMATIQUE DE LA SUSPENSION SCOLAIRE}

Si l'on s'entend sur le fait qu'au préscolaire et au primaire, les conflits entre les élèves semblent inévitables et que leurs origines tout autant que leurs causes sont multiples, les comportements violents qui en découlent entraînent généralement une conséquence unique: la suspension scolaire. Il y a cependant tout lieu de se demander si cette mesure disciplinaire est appropriée à l'enfant de 3 à 9 ans. Permet-elle de contrer ses comportements violents envers les pairs à l'école ou risque-t-elle, au contraire, d'aggraver la situation-problème en entravant son processus de scolarisation et de socialisation? D' où la dernière question de recherche, à savoir : Quelles sont les mesures disciplinaires appliquées par l'école permettant de contrer les comportements violents des 3 à 9 ans et de ne pas entraver leur processus de scolarisation et de socialisation? Le présent chapitre tente d'apporter des pistes de réponse à cette question.

\subsection{L'ORIGINE DE LA MESURE DISCIPLINAIRE}

Tout semble indiquer que la suspension scolaire est devenue une pratique pédagogique courante particulièrement depuis quarante ans, soit depuis que l'école a cessé de recourir à des châtiments corporels comme mesure disciplinaire, suite à des comportements jugés inappropriés. Largement répandue malgré le manque de connaissances sur ses effets en bas âge, cette mesure disciplinaire est actuellement appliquée non seulement en réponse aux comportements violents, mais également pour des motifs de 
non-respect du code de conduite. Selon Van Neste (1994), ce contexte peut expliquer à lui seul le nombre de plus en plus élevé d'élèves suspendus à répétition, et ce, pour des raisons parfois banales, tant et si bien que la suspension fait régulièrement l'objet de remises en question, sans pour autant être abandonnée.

\subsection{DES DÉFINITIONS}

Avant de poursuivre, il convient de préciser quelques termes nous permettant de comprendre les différences entre l'exclusion et la suspension. L'exclusion est un concept général qui renvoie à l'interdiction faite à un élève de participer aux activités scolaires, à la suite de comportements jugés non appropriés. Elle peut être totale ou partielle. La suspension scolaire est une forme d'exclusion faisant suite à une situation-problème qui demande une intervention rapide. D'une durée prédéterminée, la suspension est une mesure disciplinaire généralement prévue au code de conduite de l'école. Deux formes de suspension scolaire sont actuellement en vigueur dans les écoles canadiennes: la suspension interne et la suspension externe. Lorsqu'un élève fait l'objet d'une suspension interne, il est exclu de la classe pour être relocalisé dans un endroit prévu à cette fin ou, en l'absence d'une telle ressource, être envoyé au bureau de la direction d'école. Quant à l'élève faisant l'objet d'une suspension externe, il est exclu de l'école pour se retrouver en milieu familial. Le retour à l'école fait généralement l'objet d'une procédure administrative impliquant le parent, le personnel enseignant, la direction d'école et l'élève.

\subsection{LES AVANTAGES ET LES INCONVÉNIENTS DE LA SUSPENSION SCOLAIRE}

Que la suspension scolaire soit interne ou externe, ses défenseurs fondent leur argumentation sur la protection de l'ordre et des droits de la majorité des élèves. À ce titre, il est généralement reconnu que les élèves ayant des comportements violents envers leurs pairs au préscolaire et au primaire ont une influence négative sur les relations interpersonnelles (Lauwrence et Hayden, 1995). De plus, ces élèves nuisent au climat d'apprentissage dans la salle de classe et contribuent significativement à l'augmentation du niveau de stress chez les enseignants (Imich, 1994), ce qui prive la majorité des élèves de recevoir un enseignement de qualité. Lors de situations de crise, la suspension scolaire permet alors à l'enseignant et aux élèves de la classe, de même qu'à l'élève suspendu, d'apaiser temporairement les tensions (Garibaldi, Blanchard et Brooks, 1996). 
Quant aux opposants à la suspension scolaire, ils maintiennent que lorsqu'il y a protection des besoins éducationnels et des droits de la majorité des élèves, cette protection doit s'étendre à la minorité d'élèves ayant des comportements violents (Mellard et Seybert, 1996). Ainsi, un tel comportement s'entend non seulement comme une condition perturbatrice pour l'ensemble des élèves, mais également comme une limite au développement scolaire et social de l'élève suspendu (Hayden et Ward, 1996). Berkowitz (1993, p. 122) définit cette limite comme étant «une absence de contrôle interne permettant à l'élève de s'ajuster face aux demandes formulées par l'école et l'absence de compétences permettant à celle-ci d'y répondre adéquatement». Quoi qu'il en soit, dans la grande majorité des cas, la suspension externe est le reflet d'une discipline déficiente et de tensions élevées dans l'univers scolaire (Uchitelle, Bartz et Hillman, 1989).

Même si on peut établir un lien entre l'application de la suspension scolaire et les nouvelles approches de protection et de sécurité préconisées tant par le ministère fédéral de la Justice que par le ministère de l'Éducation de l'Ontario, cette mesure coercitive se révèle rapidement une solution temporaire à un problème beaucoup plus complexe (Hayden, 1994). Il paraît donc crucial que la suspension n'entrave pas le développement socioscolaire des enfants (Conseil canadien de développement social, 2001; Royer, 1995), à plus forte raison s'ils sont aux prises avec une problématique de comportements violents envers leurs pairs au préscolaire et au primaire.

Bien que la suspension scolaire externe soit une mesure dont l'application est dorénavant encadrée par des lois, règlements et politiques tant en Ontario qu'au Canada, il y a tout lieu de se demander si elle s'avère efficace dans sa forme actuelle, c'est-à-dire lorsqu'elle est appliquée de manière uniforme et sans distinction pour l'ensemble des comportements violents des élèves. Il y a également lieu de s'interroger si elle permet aux élèves de 3 à 9 ans de surmonter leur situation-problème, tout en leur permettant de faire l'apprentissage de compétences scolaires et sociales nécessaires au développement de relations plus harmonieuses avec leurs pairs, voire de s'adapter aux multiples exigences de l'école. Finalement, en l'absence de réponses positives, il y a tout lieu de chercher à savoir quelles sont les mesures disciplinaires les plus appropriées à l'enfant de 3 à 9 ans.

\section{LES PERCEPTIONS DES PARENTS FACE À LA SUSPENSION SCOLAIRE EXTERNE}

En dépit de l'entrée en vigueur des nouvelles mesures légales encadrant la suspension scolaire externe en Ontario, le deux tiers des parents de l'échantillon se déclarent en désaccord avec son application en réponse aux 
comportements violents de leur enfant envers ses pairs au préscolaire et au primaire. Parmi les autres parents, un sur cinq s'avère plus ou moins en accord et un sur dix se dit d'accord. Finalement, quelques parents seulement déclarent ne pas avoir d'opinion précise sur le sujet. Aucun enfant de ces derniers n'a toutefois vécu l'application de la mesure disciplinaire, mais seulement, une possibilité que celle-ci soit appliquée éventuellement. Les perceptions des parents face à la suspension scolaire externe sont présentées au tableau 5.1.

TABleau 5.1

Les perceptions des parents face

à la suspension scolaire externe $(n=60)$

\begin{tabular}{lcccc}
\hline & \multicolumn{2}{c}{ Mères } & \multicolumn{3}{c}{ Pères } \\
\cline { 2 - 5 } & $N$ & $\%$ & $N$ & $\%$ \\
\cline { 2 - 5 } & 54 & 90,0 & 6 & 10,0 \\
\hline Sont en désaccord & 34 & 56,5 & 5 & 8,5 \\
\hline Sont plus ou moins en accord & 10 & 16,5 & 1 & 1,5 \\
\hline Sont d'accord & 6 & 10,0 & & \\
\hline N'ont pas d'opinion précise & 4 & 6,5 & & \\
\hline
\end{tabular}

\subsection{LES MOTIFS DU DÉSACCORD}

Tous les parents qui se déclarent en désaccord avec l'application de la suspension scolaire externe croient que cette mesure disciplinaire ne contribue pas à améliorer les comportements violents de leur enfant. Au contraire, ils laissent même entendre qu'elle suscite l'effet inverse: Je ne pense pas que le système qu'ils ont, de punir constamment les enfants et de les rejeter, non, ça ne donne rien, tant qu'à moi, ça empire la situation (11); Tout ce que ça cause, c'est que le problème progresse plus vite et devient plus gros (48); Ça fait juste escalader (51); Ça peut juste être pire (112).

La majorité des parents de ce sous-échantillon mentionnent que le fait de ne pas avoir été consultés avant que l'école prenne la décision de suspendre leur enfant à l'externe s'avère des plus inappropriés puisqu'une telle décision désorganise le milieu familial: L'école t'envoie un papier qui mentionne la suspension et c'est fini. T'as même pas le temps de faire des plans (24); L'école l'a fait sans même m'en parler (47); Ils m'ont appelé pour dire de venir chercher mon enfant immédiatement puisqu'il était suspendu (74); Mon enfant m'a dit qu'il était suspendu pour trois jours lorsqu'il est arrivé à la maison (85). 
En ce sens, la manière dont la suspension scolaire externe a été annoncée au quart des parents a contribué à leur faire ressentir un grand inconfort, de même qu'à causer un réel tort moral à leur enfant: Dans le contexte dans lequel ç'a été présenté, c'était un brin intimidant parce que, je peux comprendre que même si l'éducation de l'enfant c'est notre responsabilité, je me suis sentie comme poignée au collet (06); Le directeur a dit que mon enfant ne pourrait plus venir à l'école [...] à sa première journée d'école. C'était un gros coup à prendre pour mon enfant. Il était très traumatisé. Il s'est mis en dessous d'un bureau et il a commencé à pleurer (44); La suspension a été très dure pour mon enfant... (62); Un enfant de trois ans, veux-tu bien me dire ce qu'il a fait de si mal pour le rejeter comme ça (109); À quatre ans, c'est plus parce qu'ils veulent se débarrasser d'elle (112).

Une fois l'annonce faite, même après que les parents ont manifesté leur désaccord avec la décision de suspendre leur enfant à l'externe, l'école n'a pas respecté leur opinion dans le quart des cas: J'ai donné mon avis et on s'en est foutu comme de l'an quarante (11); Ils m'ont averti par téléphone. J'ai dit ce que j'en pensais mais ils n'ont pas voulu changer d'idée (46); La direction a maintenu la suspension d'une journée malgré mon désaccord (52); On m'a pas donné le choix (74).

Seulement trois parents de ce sous-échantillon ont réussi à transformer la suspension externe en suspension interne: Mon enfant a eu une menace de suspension externe parce qu'il ne voulait pas coopérer avec ses pairs et les adultes. Mais il ne l'a pas eue, parce que je n'étais pas d'accord avec cette option. Il a eu seulement une suspension interne (10); Il se faisait suspendre à l'externe. Ils m'ont demandé ce que j'en pensais. Comme je n'étais pas d'accord, elle a retiré mon enfant de la classe et l'a suspendu à l'interne (51).

De plus, malgré le désaccord qu'avaient exprimé quatre parents, l'école a utilisé des menaces pour maintenir l'application de la suspension scolaire externe: Pour me faire accepter la suspension d'une journée, la direction $m^{\prime}$ a menacée d'imposer trois jours au prochain comportement inacceptable de mon fils. C'est comme du chantage. Finalement, ils font ce qu'ils veulent et nous font des menaces si on ne l'accepte pas (52); Ils m'ont dit, soit tu acceptes qu'on le suspende, soit on le «renvoie»! (109).

Pour un peu moins du tiers des parents, le fait que la décision de suspendre à l'externe est imposée par l'école, qu'elle va à l'encontre de leur volonté et qu'il est impossible de la renverser leur donne un sentiment d'impuissance et d'incompréhension: On peut pas faire grand-chose, nous autres. On est juste des numéros (06); On n'a pas de chances les parents. Ils ne nous écoutent pas (48); Ils m'ont envoyé une feuille où il était écrit que mon fils était suspendu. Si je ne la signais pas, ils l'auraient suspendu de toute manière (85); La direction d'école, y comprennent pas les parents (96). 
Par ailleurs, quatre parents sur cinq de ce sous-échantillon ne croient pas à l'efficacité de la suspension scolaire externe telle qu'elle est appliquée actuellement dans les écoles, puisque la mesure disciplinaire ne permet pas de s'attaquer à la source du problème: Ma fille a un problème de «parlage» [...]. La suspension externe est frustrante parce qu'elle représente l'incompréhension de ses problèmes réels (09); Bien, tout ce qu'ils font, c'est suspendre, suspendre, suspendre, parce qu'ils trouvent pas d'autre solution. Ils n'essaient pas de résoudre le problème en profondeur (112). Au contraire, plus de la moitié des parents jugent que son application à répétition s'avère des plus inefficaces: C'est tout le temps les mêmes qui sont suspendus, ceux qui ont des problèmes. À quoi ça rime de faire ça? Ça donne aucun résultat (10.1); L'école suspend trop souvent mon enfant. Des semaines de temps que ça dure, et ça n'améliore pas la situation (51).

Selon plus de la moitié des parents, la suspension scolaire externe s'avère toujours très dommageable au plan de la scolarisation de leur enfant: L'enfant est exclu des apprentissages (10); La suspension accélère les problèmes d'estime de soi de l'enfant et ses difficultés d'apprentissage dans les matières scolaires (48); Les élèves prennent de plus en plus de retard et l'enseignante s'en lave les mains (52); Si les enfants sont déjà en arrière à cause de leur comportement, ça les met encore plus en arrière (85). Ces parents soutiennent également qu'elle s'avère très dommageable au plan de la socialisation de l'enfant: L'isolement de mon enfant amène un manque au niveau de son apprentissage social (11); Le fait de suspendre l'enfant, ça représente la première étape pour qu'il se sente exclu et qu'il n'aille pas dans le droit chemin plus tard (48); La suspension a été néfaste parce que la première chose, il a été retiré de ses amis. Sans explication (109).

La moitié des parents pensent que leur enfant est trop jeune pour en saisir toute la portée. Douze de ces 19 parents ont un enfant âgé de 6 ans ou moins: À son âge, il n'a vraiment pas compris (04); Mon enfant ne comprend pas les conséquences de ses actions. Une journée sans aller à l'école n'est pas assez rapide suite à un mauvais comportement. Il y a trop d'attente entre l'action et la punition (22); Mon enfant ne sait même pas ce que ça veut dire être suspendu (92); Ça lui passe par-dessus la tête, parce qu'il ne s'en rappelle même pas! (110).

Pour deux parents sur cinq, au lieu d'avoir un effet punitif, la mesure disciplinaire apparaît même plaisante aux yeux de leur enfant: C'est une journée de vacances avec sa maman (01); Je suis contre, parce que mon enfant est heureux de cette situation (13); Il pense que c'est une journée de congé (50); $C^{\prime} e s t$ amusant parce qu'il n'est pas obligé de faire ses travaux scolaires (58); Pour lui, rester à la maison, c'est une "game». Et il aime ça jouer (81); Ça lui fait plaisir de pas y aller (91). 
Pour garder une certaine crédibilité aux yeux de leur enfant, selon le tiers des parents, la mesure disciplinaire leur apparaît injustifiée lorsqu'elle est appliquée pour des motifs banals: Des retenues, ça c'est normal, mais pas te faire suspendre de l'école pour des petites choses (102); Je veux dire pas si c'est juste l'attitude ou juste verbal. Non, je trouve qu'il y a d'autres façons que celle de suspendre (106); Suspendre juste parce qu'elle n'écoute pas toujours, là, c'est pas correct (112).

Pour le tiers des parents, cette situation peut même encourager la récidive des comportements violents chez leur enfant: Elle n'a qu'à péter une crise pour pouvoir venir à la maison (09); Ça risque d'avoir l'effet contraire et d'encourager les mauvais comportements (11); Il sait quoi faire pour avoir une suspension (51); Je pense que ça va amplifier ses comportements plus qu'autre chose [...]. En lui indiquant la porte de sortie, il va l'utiliser plus souvent. Ça va devenir une façon d'aller à la maison (90); Il sait que s'il fait un mauvais coup, je viendrai le chercher (97).

Pour contrer le risque de récidive inhérent à l'effet positif de la suspension scolaire externe, le quart des parents proposent que l'école applique des mesures disciplinaires à l'interne qui soient contraignantes: Il me semble qu'il aurait pu avoir d'autre chose comme des excuses, s'arranger pour qu'il ait un peu honte (12); Relire le code de conduite de l'école, faire de la lecture supplémentaire et un peu de nettoyage (96); Une suspension pour moi, c'est... des travaux forcés. Ça a l'air ridicule, mais ils pourraient ramasser, faire du ménage à l'école (97); Lui enlever une activité spéciale: ça, ça peut faire mal (106).

Deux autres parents proposent d'appliquer des mesures positives: Je leur ai demandé de lui donner une tâche qui l'aiderait à se sentir revalorisé, comme de laver les tableaux (21); Je leur ai proposé de montrer quelques notes de piano à mon fils. Il adore la musique. Je leur ai dit que ça pouvait être une forme de motivation. L'école a un piano dans le gymnase qui ne sert jamais. Ils n'ont jamais mis cette solution en pratique (48).

Un parent sur cinq de ce sous-échantillon se résigne malgré sa vive opposition à accepter la suspension scolaire externe parce que l'école l'a informé qu'il s'agit d'une loi qui doit être appliquée de manière uniforme et sans distinction pour l'ensemble des élèves: Même si c'était un événement isolé, elle devait quand même appliquer la politique (01); On donne une suspension. On dit que c'est la loi, qu'il faut le faire (12); L'école m'a dit qu'il était suspendu parce que c'était les règlements de l'école et du conseil. C'était obligatoire et ils ne pouvaient pas faire autrement (83); Ils devaient appliquer la suspension pour respecter la loi de l'Ontario (112).

Pour que la suspension scolaire externe soit mieux acceptée, des parents soutiennent qu'il serait important que l'école applique un système de mesures disciplinaires graduelles où elle ne serait utilisée qu'en dernier 
ressort: Le directeur aurait pu utiliser d'autres techniques avant d'en arriver là. Ça aurait été plus d'ouvrage pour lui. C'est facile de renvoyer (12); Sans avertissement, c'est vraiment une mesure exagérée (52).

Près d'un parent sur cinq considère que la suspension externe représente un transfert de responsabilité du milieu scolaire vers le milieu familial: Le problème se passe à l'école et il devrait rester à l'école (12); Je trouve que l'enseignante ne fait pas sa «job». On devrait lui couper son salaire lorsqu'elle met l'enfant en dehors de la classe. Elle serait bien plus motivée pour le garder (48). D'une certaine manière, ce transfert exige à ces parents de transférer à leur tour leurs responsabilités parentales à une tierce partie, ce qui ne se fait pas sans heurt: Il se ramasse chez une gardienne et c'est $d u$ «je-m'enfoutisme» (10); Comment je fais, moi, comme parent, pour exiger de ma gardienne qu'elle exige de mon enfant qu'il fasse ses choses? (21); Si ça ne va pas à l'école, ça veut pas dire que ça va aller mieux chez la gardienne (109).

D'autres parents soulignent aussi comme inconvénient que la suspension externe leur occasionne davantage de désagréments à eux qu'à leur enfant, car cette mesure disciplinaire les oblige à s'absenter du travail et les force à modifier un horaire déjà chargé: C'est moi qui manque une journée de travail (01); La suspension ne punit pas l'enfant, mais plutôt les parents qui ne peuvent pas aller travailler (13); Des fois, c'est un problème de savoir qui de nous deux va prendre la journée «off» [de congé]. Des fois, on est mal pris! (96); Ça n'aide pas les parents, ça, c'est sûr et certain quand tu travailles (97); C'est un problème parce que c'est encore un changement de routine (111).

Ces parents ne trouvent pas leur compte avec la suspension scolaire externe, car ils évaluent qu' elle profite davantage à l'école: Ça aide à l'interne, les professeurs et la direction, de faire une suspension. Ils ont la paix pendant qu'il n'est pas là. Mais, à part eux qui ont des avantages clairs et marqués, je ne pense pas que pour l'enfant, c'est un avantage (11.1); Ça rend plus service à l'école qu'à nous (44); En attendant qu'on trouve un moyen, je l'ai sorti de la classe pendant un mois parce que ça n'allait pas. Et c'était au grand soulagement des enseignants et de la direction (51).

Sur le plan des attitudes de leur enfant, quelques parents ont observé que la mesure disciplinaire contribue à les démotiver tout en favorisant leur désintérêt pour l'école: Mon enfant ne voulait pas retourner à l'école après la suspension (09); C'est absolument zéro sur l'enfant, car il ne veut plus retourner à l'école. De mon côté, je dois travailler deux fois plus fort pour l'encourager à y retourner. Ce n'est pas envisageable qu'un enfant soit «dropout» en première année! (48).

Afin de faire face à la situation, quelques parents adoptent d'abord des comportements de résistance. Puis, ils s'y adaptent en maintenant un certain contrôle: J'ai résisté autant que j'ai pu à ce qu'ils voulaient me faire faire. 
Finalement, j'ai accepté de donner du Ritalin ${ }^{\circledR}$ à mon fils pour qu'il soit réintégré en classe. Mais, je veux pas lui en donner trop. Je veux pas le rendre zombie. C'est moi qui contrôle le dosage à l'école. Ils n'ont rien en surplus (48); On est d'accord pour essayer le Ritalin ${ }^{\circledR}$ pour cinq ou six mois. Après ça, graduellement, on va l'enlever (93).

À ces difficultés, s'ajoutent les problèmes de communication de toute nature que des parents disent avoir éprouvés avec le personnel scolaire: J'aurais aimé savoir qu'il y avait des problèmes avant que ça devienne assez grave, qu'ils le mettent à la porte (97).

Un parent sur dix note que l'école est aux prises avec un besoin criant de ressources humaines spécialisées qu'ils pourraient consulter en situation de crise, et ce, avant d'envisager la suspension scolaire externe: Il devrait y avoir un système où un psychologue intervient tout de suite. Il y a vraiment un manque de personnel à ce niveau-là (85); Ils n'ont pas de conseillère. Ils n'ont personne pour lui parler puis l'aider (97).

En raison du manque de personnel spécialisé œuvrant au préscolaire et au primaire, ces parents trouvent que la réinsertion de leur enfant en salle de classe, suite à la suspension scolaire externe, est interminable: Le retour à l'école a été très lent. Ma fille allait à l'école trois heures et demie par jour seulement, pendant trois semaines (09).

Finalement, pour d'autres parents, le retour en classe suite à la suspension scolaire externe ne change rien à la situation-problème de leur enfant, qui demeure entière: Depuis le retour à l'école, c'est comme... tout est à recommencer (91). Les motifs des parents en désaccord avec la suspension scolaire externe sont présentés au tableau 5.2.

TABLEAU 5.2

\section{Les motifs des parents en désaccord avec la suspension scolaire externe $(n=39)$}

\begin{tabular}{lcccc}
\hline & \multicolumn{2}{c}{ Mères } & \multicolumn{2}{c}{ Pères } \\
\cline { 2 - 5 } & $N$ & $\%$ & $N$ & $\%$ \\
\cline { 2 - 5 } & 34 & 87,0 & 5 & 13,0 \\
\hline $\begin{array}{l}\text { Croient qu'elle n'améliore pas } \\
\text { les comportements violents }\end{array}$ & 34 & 87,0 & 5 & 13,0 \\
\hline $\begin{array}{l}\text { N'ont pas été consultés avant } \\
\text { son application }\end{array}$ & 32 & 82,0 & 4 & 10,5 \\
\hline Ne croient pas à son efficacité & 29 & 74,5 & 3 & 7,5 \\
\hline $\begin{array}{l}\text { Croient qu'elle ne règle pas la } \\
\text { situation-problème à la source }\end{array}$ & 29 & 74,5 & 3 & 7,5 \\
\hline
\end{tabular}


Tableau 5.2 (suite)

\section{Les motifs des parents en désaccord avec la suspension scolaire externe $(n=39)$}

\begin{tabular}{|c|c|c|c|c|}
\hline & \multicolumn{2}{|c|}{ Mères } & \multicolumn{2}{|c|}{ Pères } \\
\hline & $N$ & $\%$ & $N$ & $\%$ \\
\hline $\begin{array}{l}\text { Croient que l'application } \\
\text { à répétition est inefficace }\end{array}$ & 23 & 59,0 & 3 & 7,5 \\
\hline $\begin{array}{l}\text { Croient qu'elle s'avère } \\
\text { dommageable à la scolarisation }\end{array}$ & 18 & 46,0 & 3 & 7,5 \\
\hline $\begin{array}{l}\text { Croient qu'elle s'avère } \\
\text { dommageable à la socialisation }\end{array}$ & 18 & 46,0 & 3 & 7,5 \\
\hline Trouvent leur enfant trop jeune & 17 & 43,5 & 2 & 5,0 \\
\hline $\begin{array}{l}\text { Pensent qu'elle apparaît plaisante } \\
\text { aux yeux de l'enfant }\end{array}$ & 15 & 38,5 & 1 & 2,5 \\
\hline $\begin{array}{l}\text { Pensent qu'elle est injustifiée } \\
\text { pour des motifs banals }\end{array}$ & 12 & 31,0 & 2 & 5,0 \\
\hline $\begin{array}{l}\text { Considèrent qu'elle encourage } \\
\text { la récidive chez l'enfant }\end{array}$ & 11 & 28,0 & 1 & 2,5 \\
\hline $\begin{array}{l}\text { Ressentent de l'impuissance } \\
\text { et de l'incompréhension }\end{array}$ & 11 & 28,0 & & \\
\hline $\begin{array}{l}\text { N’ont pas été respectés } \\
\text { dans leur opinion }\end{array}$ & 9 & 23,0 & 1 & 2,5 \\
\hline $\begin{array}{l}\text { Éprouvent de l'inconfort lors } \\
\text { de son annonce }\end{array}$ & 8 & 20,5 & 1 & 2,5 \\
\hline $\begin{array}{l}\text { Trouvent qu'elle crée un tort } \\
\text { moral à l'enfant }\end{array}$ & 8 & 20,5 & & \\
\hline $\begin{array}{l}\text { Proposent l'application } \\
\text { de mesures contraignantes }\end{array}$ & 7 & 18,0 & 2 & 5,0 \\
\hline $\begin{array}{l}\text { Se résignent à l'accepter malgré } \\
\text { leur vive opposition }\end{array}$ & 7 & 18,0 & 1 & 2,5 \\
\hline $\begin{array}{l}\text { Pensent qu'elle devrait être utilisée } \\
\text { en dernier ressort }\end{array}$ & 6 & 15,5 & 1 & 2,5 \\
\hline $\begin{array}{l}\text { Considèrent qu'elle est un transfert } \\
\text { de responsabilités }\end{array}$ & 6 & 15,5 & 1 & 2,5 \\
\hline $\begin{array}{l}\text { Sont obligés à leur tour d'effectuer } \\
\text { un tel transfert }\end{array}$ & 6 & 15,5 & & \\
\hline $\begin{array}{l}\text { Évaluent qu'elle profite davantage } \\
\text { au personnel scolaire }\end{array}$ & 6 & 15,5 & & \\
\hline $\begin{array}{l}\text { Observent qu'elle démotive et } \\
\text { crée un désintérêt chez l'enfant }\end{array}$ & 6 & 15,5 & & \\
\hline $\begin{array}{l}\text { Trouvent qu'elle leur occasionne } \\
\text { des désagréments }\end{array}$ & 4 & 10,5 & 2 & 5,0 \\
\hline
\end{tabular}




\section{TABLEAU 5.2 (suite) \\ Les motifs des parents en désaccord avec la suspension scolaire externe $(n=39)$}

\begin{tabular}{lcccc}
\hline & \multicolumn{2}{c}{ Mères } & \multicolumn{2}{c}{ Pères } \\
\cline { 2 - 5 } & $N$ & $\%$ & $N$ & $\%$ \\
\cline { 2 - 5 } $\begin{array}{l}\text { Adoptent des comportements } \\
\text { de résistance }\end{array}$ & 4 & 10,5 & 1 & 2,5 \\
\hline $\begin{array}{l}\text { S'adaptent à la situation en } \\
\text { maintenant un contrôle }\end{array}$ & 4 & 10,5 & 1 & 2,5 \\
\hline $\begin{array}{l}\text { Éprouvent des problèmes } \\
\text { de communication }\end{array}$ & 4 & 10,5 & 1 & 2,5 \\
\hline $\begin{array}{l}\text { Se sont fait menacer pour } \\
\text { l'accepter }\end{array}$ & 4 & 10,5 & & \\
\hline $\begin{array}{l}\text { Notent le besoin de ressources } \\
\text { humaines spécialisées }\end{array}$ & 4 & 10,5 & & \\
\hline $\begin{array}{l}\text { Trouvent que la réinsertion } \\
\text { en classe est interminable }\end{array}$ & 4 & 10,5 & & \\
\hline $\begin{array}{l}\text { Ont réussi à la transformer } \\
\text { en suspension interne }\end{array}$ & 3 & 7,5 & & \\
\hline $\begin{array}{l}\text { Croient davantage à des } \\
\text { mesures positives }\end{array}$ & 2 & 5,0 & \\
\hline $\begin{array}{l}\text { Trouvent que le retour en classe } \\
\text { ne règle rien }\end{array}$ & 2 & 5,0 & \\
\hline
\end{tabular}

\subsection{L'AMBIVALENCE CHEZ LES PLUS OU MOINS EN ACCORD}

Bien qu'ils soient plus ou moins en accord avec la suspension externe, un peu plus de la moitié des parents évaluent que, dans le meilleur intérêt de leur enfant, il vaut mieux ne pas s'opposer aux décisions déjà prises par l'école: Au téléphone, la direction a dit que ça ne pouvait plus continuer comme ça et que la suspension s'en venait. J'ai dit que j'étais d'accord. J'avais pas tellement le choix (28); Ils m'ont demandé ce que j'en pensais, mais je ne voulais pas être contre eux, car mon enfant a un problème et c'est comme ça qu'ils règlent ça (98).

Un peu plus de la moitié des parents de ce sous-échantillon croient qu'elle serait peut-être appropriée, mais pas à un jeune âge. Trois de ces six parents ont un enfant de 6 ans et moins: On ne devrait pas utiliser ça avant la première ou la deuxième année, et seulement dans les cas sérieux (20); Je trouve qu'à 9 ans, c'est trop jeune pour suspendre (28); $\grave{A}$ son âge, dans le moment, ça ne lui aide pas du tout (98); Non, pas du tout. Pas à 4 ans. Peut-être plus tard, mais pas à 4 ans (108). 
À l'inverse, un peu moins du tiers des parents semblent croire que plus la suspension scolaire externe survient jeune, plus elle a des chances de fonctionner avec l'enfant: Je pense que plus jeune, ça peut avoir un effet parce qu'ils ont peur de ça (37); À son âge, je crois que ça marche parce qu'il n'a pas refait ce qu'il avait fait. Plus tard, ils sont plus contents qu'autre chose (82).

Selon près de la moitié des parents de ce sous-échantillon, cette mesure pourrait convenir davantage à certains enfants qu'à d'autres, tout dépendant de leur tempérament et de leur personnalité: Peut-être pour certains enfants plus sensibles, une suspension devient comme un désastre dans son monde à lui. Mais pour mon enfant, ce n'était aucunement un désastre (02); Pour être efficace, ça dépend du caractère de l'enfant (05); Ça peut avoir un effet, car il adore aller à l'école. En ce moment, c'est un atout qu'on a réussi à garder. Je dois dire qu'à ce niveau-là, c'est valable (20).

Par ailleurs, deux parents se sont laissé convaincre par la direction d'école que cette mesure disciplinaire n'était pas mauvaise en soi pour l'enfant: Le soir, j'ai parlé à la directrice qui m'a dit de ne pas m'en faire avec ça (05); La directrice m'a rassurée que ça fait partie du développement de l'enfant et qu'il n'y a rien de problématique là-dedans (108). Les motifs des parents plus ou moins en accord avec la suspension scolaire externe sont présentés au tableau 5.3.

TABleAu 5.3

Les motifs des parents plus ou moins en accord avec la suspension scolaire externe $(n=11)$

\begin{tabular}{lcccc}
\hline & \multicolumn{2}{c}{ Mères } & \multicolumn{2}{c}{ Pères } \\
\cline { 2 - 5 } & $N$ & $\%$ & $N$ & $\%$ \\
\cline { 2 - 5 } & 10 & 91,0 & 1 & 9,0 \\
\hline $\begin{array}{l}\text { Pensent qu'il vaut mieux ne pas } \\
\text { s'y opposer }\end{array}$ & 5 & 45,5 & 1 & 9,0 \\
\hline $\begin{array}{l}\text { Croient qu'elle peut être } \\
\text { appropriée, mais plus tard }\end{array}$ & 5 & 45,5 & 1 & 9,0 \\
\hline $\begin{array}{l}\text { Trouvent qu'elle convient mieux } \\
\text { à certains enfants }\end{array}$ & 4 & 36,5 & 1 & 9,0 \\
\hline $\begin{array}{l}\text { Croient qu'elle a des chances } \\
\text { de fonctionner en bas âge }\end{array}$ & 2 & 18,0 & 1 & 9,0 \\
\hline $\begin{array}{l}\text { Se sont laissé convaincre qu'elle } \\
\text { n'est pas mauvaise en soi }\end{array}$ & 2 & 18,0 & & \\
\hline
\end{tabular}




\section{3. ÊtRE D'ACCORD TOUT EN FORMULANT CERTAINS SOUHAITS}

Parmi les parents qui se disent $\mathrm{d}^{\prime}$ accord avec la suspension scolaire externe, la moitié d'entre eux croient qu'elle peut contribuer à responsabiliser leur enfant face aux conséquences de ses gestes: Il y a des coups de pied pour lesquels l'enfant doit subir la responsabilité (07); Il doit subir très jeune les conséquences de ses mauvais comportements. Ça l'aidera à devenir un meilleur adulte (23); Dans le fond, la suspension, c'est l'enfant qui doit la payer. C'est là où la suspension, oui, je suis d'accord avec ça. Oui, je peux comprendre que vous voulez donner une leçon, mais ce ne doit pas être le parent qui la paie. Ce doit être l'enfant (99).

Le tiers des parents de ce sous-échantillon pensent que la suspension scolaire externe peut s'avérer l'élément déclencheur leur permettant d'amorcer un processus de résolution de la situation-problème: Ça $m^{\prime} a$ indiqué qu'il était temps que je me réveille, que ça me pousse à faire quelque chose (12.1); Il y a plusieurs solutions à un problème, puis la changer d'école c'était juste une des solutions, puis c'était une bonne solution (114).

Bien que ces parents se disent d'accord avec l'application de la mesure disciplinaire, le tiers d'entre eux souhaiteraient avoir une meilleure connaissance de ses effets à long terme: J'aimerais ça savoir si ça va l'aider tant que ça (14); Je ne sais pas si ça va faire de quoi. J'ai aucune idée, comme j'aimerais ça faire des rencontres, en parler (99). Les motifs des parents en accord avec la suspension scolaire externe sont présentés au tableau 5.4.

TABleaU 5.4

Les motifs des parents en accord avec la suspension scolaire externe $(n=6)$

\begin{tabular}{lcccc}
\hline & \multicolumn{2}{c}{ Mères } & \multicolumn{2}{c}{ Pères } \\
\cline { 2 - 5 } & $N$ & $\%$ & $N$ & $\%$ \\
\cline { 2 - 5 } & 6 & 100,0 & 0 & 0,0 \\
\hline $\begin{array}{l}\text { Croient qu'elle peut } \\
\text { responsabiliser l'enfant }\end{array}$ & 3 & 50,0 & & \\
\hline $\begin{array}{l}\text { Pensent qu'elle peut résoudre } \\
\text { la situation-problème }\end{array}$ & 2 & 33,5 & & \\
\hline $\begin{array}{l}\text { Souhaiteraient mieux connaître } \\
\text { ses effets à long terme }\end{array}$ & 2 & 33,5 & & \\
\hline
\end{tabular}




\subsection{NE PAS AVOIR D'OPINION PRÉCISE SUR LE SUJET}

Tous les parents n'ayant pas d'opinion précise au sujet de la suspension scolaire expliquent que c'est parce qu'ils ne l'ont pas encore expérimentée: Je n'ai jamais connu la suspension externe, mais seulement une possibilité, parce qu'il a un comportement pas mal agressif. Je ne peux pas vraiment dire si ça aiderait ou pas (86); Aucune idée. J'ai pas de commentaires parce que j'ai jamais été obligée de sévir à propos de ça (107).

Bien qu'ils n'aient pas d'opinion précise au sujet de cette mesure, la moitié des parents de ce sous-échantillon trouvent primordial d'être consultés avant qu'elle ne soit mise en application: Je comprends qu'ils suivent certaines étapes. Je donnerais mon opinion quand même. Je suivrais les indications de la suspension, mais j'essaierais de parler avec l'enseignante et le directeur, en espérant que ça n'arrive plus (30); Même s'ils ont des règles à suivre, l'école devrait toujours me demander mon opinion avant de passer à l'action (32). Les motifs des parents n'ayant pas d'opinion précise sur la suspension scolaire externe sont présentés au tableau 5.5.

Tableau 5.5

Les motifs des parents qui n'ont pas d'opinion précise sur la suspension scolaire externe $(n=4)$

\begin{tabular}{lcccc}
\hline & \multicolumn{2}{c}{ Mères } & \multicolumn{2}{c}{ Pères } \\
\cline { 2 - 5 } & $\boldsymbol{N}$ & $\%$ & $\boldsymbol{N}$ & $\%$ \\
\cline { 2 - 5 } & 4 & 100,0 & 0 & 0,0 \\
\hline Ne l'ont pas encore expérimentée & 4 & 100,0 & & \\
\hline $\begin{array}{l}\text { Trouvent primordial d'être } \\
\text { consultés avant l'application }\end{array}$ & 2 & 50,0 & & \\
\hline
\end{tabular}

\section{UNE ALTERNATIVE À LA SUSPENSION SCOLAIRE EXTERNE}

Un peu plus des deux tiers des parents de la présente recherche ne croient pas que la suspension scolaire externe dans sa forme actuelle puisse s'avérer une mesure disciplinaire appropriée pour contrer les comportements violents de leur enfant. Selon eux, son application comporte davantage d'effets négatifs que positifs et se révèle dommageable au plan de la scolarisation et de la socialisation de leur enfant. 
Ces résultats se situent dans le prolongement d'études qui concluent à des appréhensions similaires chez les parents (Blyth and Milner, 1993; Hayden, 1994; Royer, Bitaudeau et Saint-Laurent, 1993). De même, à l'instar des conclusions de l'étude de Dupper (1994), la suspension scolaire externe contribue à générer un contexte conflictuel entre l'école et les parents lorsque ces derniers s'y opposent. Pour toutes ces raisons, les parents semblent privilégier la suspension scolaire interne (Farner, 1996; Guindon, 1992; Stage, 1997; Sullivan, 1989), ainsi que d'autres mesures disciplinaires mieux adaptées à la situation-problème de leur enfant.

\subsection{LA SUSPENSION SCOLAIRE INTERNE}

Plus de quatre parents sur cinq de notre échantillon privilégient la suspension scolaire interne, afin de ne pas entraver le processus de scolarisation et de socialisation de leur enfant: Moi, je me dis que, trois jours de suspension interne, ce serait plus efficace parce qu'il irait à l'école. Puis, il se ferait pas enlever le privilège de voir ses amis (05); Ça serait mieux une suspension interne où ils lui expliqueraient la situation (50); Il devrait y avoir des suspensions internes à l'école, à un moment où l'enfant peut réfléchir, comme à la récréation ou sur l'heure du dîner (74); La solution, ce serait la suspension scolaire interne. Dans ce cas-ci, il y a beaucoup de choses qui pourraient être essayées (90); Les suspensions devraient se passer à l'école, à l'interne (96).

Toutefois, selon le quart des parents, pour être efficace, la suspension interne ne devrait pas être appliquée seule, mais s'accompagner d'activités de rattrapage scolaire: Je suis d'accord avec la suspension interne, mais pas dans le bureau du directeur adjoint, parce qu'il perd son temps. Quand il n'est pas en classe, il manque tous les apprentissages. Pourquoi mon fils n'est-il pas installé dans un petit groupe de récupération quand ça va mal? (48); Tu sais, comme lui faire refaire les travaux qu'il manque dans la classe ou quelque chose comme ça (102).

Ces parents ajoutent qu' elle devrait aussi inclure des activités de prise de conscience: Moi, je pense qu'un enfant, quand il fait quelque chose de mal, il faut le faire parler pour voir pourquoi il a fait ça, puis trouver une solution avec lui (11); Ça dépend de la cause de la suspension. Si l'enfant a besoin de socialiser, la solution n'est pas de l'isoler, mais de lui parler (96). Les perceptions des parents face à la suspension scolaire interne sont présentés au tableau 5.6. 
TABLEAU 5.6

Les perceptions des parents

face à la suspension scolaire interne $(n=60)$

\begin{tabular}{lcccc}
\hline & \multicolumn{2}{c}{ Mères } & \multicolumn{2}{c}{ Pères } \\
\cline { 2 - 5 } & $\boldsymbol{N}$ & $\%$ & $\boldsymbol{N}$ & $\%$ \\
\cline { 2 - 5 } & 54 & 90,0 & 6 & 10,0 \\
\hline $\begin{array}{l}\text { Privilégient la suspension } \\
\text { scolaire interne }\end{array}$ & 49 & 81,5 & 3 & 5,0 \\
\hline $\begin{array}{l}\text { Croient qu'elle devrait } \\
\text { s'accompagner d'activités } \\
\text { de rattrapage scolaire }\end{array}$ & 15 & 25,0 & 2 & 3,5 \\
\hline $\begin{array}{l}\text { Croient qu'elle devrait inclure } \\
\text { des activités de prise }\end{array}$ & 13 & 21,5 & 2 & 3,5 \\
\hline \begin{tabular}{l} 
de conscience \\
\hline
\end{tabular} & & & & \\
\hline
\end{tabular}

\subsection{DES MESURES DISCIPLINAIRES ADAPTÉES À CHAQUE SITUATION-PROBLÈME}

Plus des deux tiers des parents estiment que l'école devrait tenter de déterminer, cas par cas, le moyen le plus efficace de contrer les comportements violents de leur enfant tout en adaptant des conséquences à l'acte selon son tempérament et sa personnalité: L'école devrait plutôt enlever quelque chose que l'enfant aime ou supprimer des privilèges (58); Il faudrait avoir un système après l'école pendant une heure pour qu'ils se rattrapent, en se sentant responsables de leurs actions (85); Je dirais d'enlever des récréations. Mon fils est un petit garçon qui aime aller dehors. Ce serait bon de lui faire perdre ce privilège pour qu'il comprenne (92).

Un peu plus de la moitié des parents croient que l'école devrait privilégier l'utilisation de moyens qui semblent bien fonctionner avec leur enfant: Il a eu une fiche de comportement et il a passé la récréation à trouver par lui-même pourquoi ça n'allait pas, d'après la méthode Hibou (12.1); Je trouve que c'est la technique 1-2-3 qui fonctionne le mieux avec lui (13); Le conseil de coopération, c'est magique. Ça fait diminuer ses comportements (90).

Finalement, un peu plus du tiers des parents estiment que le milieu scolaire aurait avantage à transposer à l'école certaines mesures disciplinaires qu'ils appliquent à la maison et qui donnent de bons résultats avec leur enfant: Ce que je trouverais important, c'est que les interventions soient concertées. Qu'on puisse le faire à l'école, ce qu'on fait à la maison. Ça, je pense que quand c'est travaillé d'une façon comme ça, on a plus de chances 
que ça marche (14.1); J'utilise l'avertissement, j'annonce une conséquence, puis j'attends comme une minute, deux minutes. Si ça marche pas, j'agis... C'est ça qui marche le mieux (108).

\subsection{LES PARENTS, DES PARTENAIRES DANS LE PROCESSUS DE PRISE DE DÉCISIONS}

Au lieu d'appliquer systématiquement la suspension scolaire, qu'elle soit interne ou externe, un peu moins du quart des parents proposent qu'on les consulte et qu' on les implique dans les décisions à prendre concernant leur enfant: Qu'ils essayent de fonctionner un peu plus en impliquant les parents (02); Ce qu'il faut faire, c'est de trouver une manière de fonctionner avec l'enfant, en collaboration avec les parents, pour savoir exactement quoi faire quand ça ne va pas à l'école (48); S'il y avait plus de comités parents-direction [...]. Quelque chose comme un vrai partenariat. En tant que parent, j'irais chercher la voix des autres parents qui sont peut-être pas capables de parler comme moi (99). Les perceptions des parents face aux autres mesures disciplinaires sont présentées au tableau 5.7.

TABleau 5.7

Les perceptions des parents

face aux autres mesures disciplinaires $(n=60)$

\begin{tabular}{lcccc}
\hline & \multicolumn{2}{c}{ Mères } & \multicolumn{2}{c}{ Pères } \\
\cline { 2 - 5 } & $N$ & $\%$ & $N$ & $\%$ \\
\cline { 2 - 5 } & 54 & 90,0 & 6 & 10,0 \\
\hline $\begin{array}{l}\text { Estiment qu'il faut identifier } \\
\text { le moyen le plus efficace }\end{array}$ & 37 & 61,5 & 4 & 6,5 \\
\hline $\begin{array}{l}\text { Suggèrent que l'école utilise } \\
\text { des moyens qui fonctionnent bien }\end{array}$ & 30 & 50,0 & 3 & 5,0 \\
\hline $\begin{array}{l}\text { Croient que l'école devrait } \\
\text { transposer certaines mesures } \\
\text { appliquées à la maison }\end{array}$ & 19 & 31,5 & 2 & 3,5 \\
\hline $\begin{array}{l}\text { Proposent qu'on les consulte } \\
\text { avant d'imposer les décisions }\end{array}$ & 13 & 21,5 & 1 & 1,5 \\
\hline $\begin{array}{l}\text { Proposent qu'on les implique } \\
\text { dans les décisions }\end{array}$ & 13 & 21,5 & 1 & 1,5 \\
\hline
\end{tabular}

En définitive, l'application de mesures disciplinaires personnalisées et la prise de décisions communes concernant la situation-problème de l'enfant nécessitent davantage d'ouverture et de souplesse que ne le permet 
le cadre actuel de la Loi sur la sécurité dans les écoles (Ministère de l'Éducation de l'Ontario, 2000) et celui du Code de conduite provincial (Ministère de l'Éducation de l'Ontario, 2001a). 


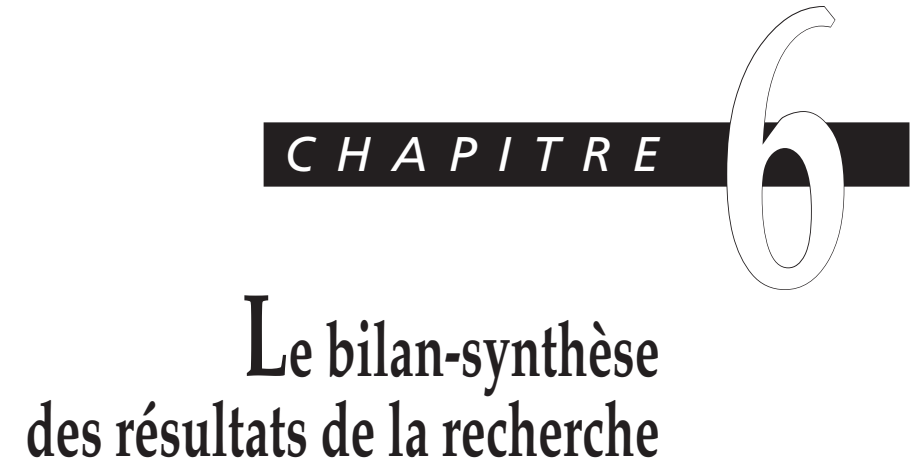



Le sixième chapitre de cet ouvrage présente le bilan-synthèse des résultats de la recherche, portant notamment sur le portrait des enfants de l'échantillon et celui de leurs parents, l'état de la collaboration entre l'école et les parents, les facteurs de soutien et les obstacles sous-jacents à la collaboration, les gestes d'empowerment posés par les parents, ainsi que leurs perceptions sur la suspension scolaire et les autres mesures disciplinaires appliquées en réponse aux comportements violents de leur enfant envers ses pairs au préscolaire et au primaire. Finalement, nous proposons des pistes éventuelles d'intervention et de recherche. Celles-ci ont notamment été puisées parmi les suggestions formulées par les parents.

\section{LE PORTRAIT DES ENFANTS}

L'échantillon d'enfants se compose de six filles et de 54 garçons. La répartition entre les sexes, soit 10\% de filles et $90 \%$ de garçons, correspond à la situation généralement décrite dans les écrits sur le sujet, à savoir que les comportements violents correspondent à une problématique davantage présente chez le sexe masculin (Kauffman, 1997). Un peu plus du tiers $\mathrm{d}^{\prime}$ entre eux sont enfants uniques alors qu'un peu moins du tiers sont des benjamins. Le statut de l'enfant dans les familles de l'échantillon est une variable dont il faudrait tenir compte dans les recherches éventuelles puisque les cadets semblent beaucoup moins concernés par la problématique des comportements violents au préscolaire et au primaire, du moins dans la présente recherche. Par ailleurs, les trois quarts des enfants de l'échantillon font preuve de violence physique, en frappant leurs pairs, tandis que les deux tiers font preuve de violence verbale avec gestes, en frappant sur des objets, ou de violence verbale sans geste, en insultant leurs pairs. Ces comportements violents étaient présents au moment de leur entrée en première année et amenaient neuf enfants sur dix de l'échantillon à être suivis par un travailleur social ou par un psychologue. Dans les mêmes proportions, ces comportements s'accompagnent d'une opposition à l'adulte. De plus, dans la majorité des cas, les enfants n'écoutent pas les consignes. Quatre enfants sur cinq éprouvent de l'anxiété et sont insécures. Également, plus de la moitié d'entre eux éprouvent des sentiments dépressifs ainsi que des problèmes de socialisation, tandis que deux enfants sur cinq manquent d'estime de soi ou de confiance en soi. De plus, le tiers d'entre eux éprouvent d'importantes difficultés d'apprentissage. Finalement, le quart des enfants de l'échantillon sont aux prises avec le Trouble du déficit de l'attention avec hyperactivité (TDAH), pour lequel ils ont obtenu médicalement une prescription de Ritalin ${ }^{\circledR}$. Le bilan-synthèse du portrait des enfants est présenté au tableau 6.1. 
Tableau 6.1

Le bilan-synthèse du portrait des enfants $(n=60)$

\begin{tabular}{|c|c|c|c|c|}
\hline & \multicolumn{2}{|c|}{ Garçons } & \multicolumn{2}{|c|}{ Filles } \\
\hline & $N$ & $\%$ & $N$ & $\%$ \\
\hline & 54 & 90,0 & 6 & 10,0 \\
\hline $\begin{array}{l}\text { Sont suivis par un travailleur } \\
\text { social ou un psychologue }\end{array}$ & 48 & 80,0 & 6 & 10,0 \\
\hline S'opposent à l'adulte & 48 & 80,0 & 5 & 8,0 \\
\hline N'écoutent pas les consignes & 46 & 76,5 & 6 & 10,0 \\
\hline $\begin{array}{l}\text { Éprouvent de l'anxiété et } \\
\text { se sentent insécures }\end{array}$ & 45 & 75,0 & 3 & 5,0 \\
\hline Font preuve de violence physique & 44 & 73,0 & 1 & 1,5 \\
\hline $\begin{array}{l}\text { Sont des enfants uniques ou } \\
\text { des benjamins }\end{array}$ & 36 & 60,0 & 3 & 5,0 \\
\hline $\begin{array}{l}\text { Font preuve de violence verbale } \\
\text { avec gestes }\end{array}$ & 35 & 58,0 & 3 & 5,0 \\
\hline $\begin{array}{l}\text { Font preuve de violence verbale } \\
\text { sans geste }\end{array}$ & 33 & 55,0 & 4 & 6,5 \\
\hline Ont des sentiments dépressifs & 32 & 53,0 & 1 & 1,5 \\
\hline Ne fonctionnent pas en groupe & 24 & 40,0 & 4 & 6,5 \\
\hline $\begin{array}{l}\text { Manquent d'estime de soi et } \\
\text { de confiance en soi }\end{array}$ & 23 & 38,0 & 5 & 8,0 \\
\hline $\begin{array}{l}\text { Éprouvent des difficultés } \\
\text { d'apprentissage }\end{array}$ & 21 & 35,0 & 2 & 3,5 \\
\hline $\begin{array}{l}\text { Avaient des comportements } \\
\text { violents dès la } 1^{\text {re }} \text { année }\end{array}$ & 21 & 35,0 & 2 & 3,5 \\
\hline $\begin{array}{l}\text { Ont le Trouble du déficit de l'attention } \\
\text { avec hyperactivité (TDAH) }\end{array}$ & 14 & 24,0 & 1 & 1,5 \\
\hline $\begin{array}{l}\text { Trouvent la transition difficile entre la } \\
\text { maternelle et la } 1^{\text {re }} \text { année }\end{array}$ & 13 & 21,5 & 2 & 3,5 \\
\hline Sont médicamentés au Ritalin ${ }^{\circledR}$ & 12 & 20,0 & 1 & 1,5 \\
\hline
\end{tabular}

\section{LE PORTRAIT DES PARENTS}

L'échantillon de parents se compose de 54 mères (dont une grand-mère) et de six pères. Les deux tiers d'entre eux possèdent un diplôme d'études secondaires ou collégiales et trois sur cinq travaillent à temps plein. La moitié des parents sont âgés de 25 à 34 ans. Enfin, le tiers des familles de l'échantillon vivent dans un contexte de pauvreté alors que les deux tiers font partie des classes moyenne et moyenne-supérieure. 
Les deux tiers des parents déclarent, afin de contrer les comportements violents de leur enfant en milieu familial, qu'ils recourent à des pratiques éducatives ayant un effet positif, notamment en faisant appel au dialogue, au retrait et à la perte de privilèges. L'analyse des données d'entrevues va dans le même sens que les résultats de recherches de Trudelle et Montambault (1994), menées au moyen d'outils psychométriques, à savoir que les parents de l'échantillon manifestent généralement un important sentiment de compétence éducative. Seulement le tiers d'entre eux déclarent utiliser des pratiques éducatives ayant un effet négatif, notamment en criant après leur enfant. En outre, un parent sur cinq déclare ne pas avoir une discipline assez stricte ou trop intervenir. Quant à la régularité des interventions, la moitié d'entre eux affirment ne pas toujours intervenir, notamment lorsqu'ils sont fatigués. Ces faibles pourcentages, par rapport à ceux dont il est question dans les principaux écrits sur le sujet, soulèvent toute la difficulté d'avoir accès aux parents d'enfants ayant des comportements violents.

Par ailleurs, l'importance accordée aux pratiques éducatives parentales (Day, Golench, MacDougall et Beals-Gonzales, 1995; Tarabulsy et Tessier, 1996; Tremblay, 2000) occulte parfois les conditions de vie et les stresseurs qu' affrontent les familles et qui affectent à leur tour les enfants. À cet égard, la moitié des parents de l'échantillon rapportent que les événements stressants que vit leur famille amplifient les comportements violents de leur enfant. Par ordre décroissant d'importance pour expliquer leur situation-problème, se trouvent les conflits intrafamiliaux, le manque de temps des parents, l'absence ou le peu de relations avec le père et la difficulté de concilier le travail et la famille.

Les stresseurs liés à l'école peuvent également expliquer les comportements violents de l'enfant. Si l'entrée à la maternelle constitue une étape importante dans sa vie, il ne faut pas sous-estimer l'entrée en $1^{\text {re }}$ année, qui l'est encore plus, et ce, en raison du changement de routine occasionné par le processus de scolarisation. À ces facteurs explicatifs s'ajoutent l'organisation scolaire trop rigide, le manque de supervision, particulièrement lors des récréations, le manque d'attention accordée à l'enfant par le personnel enseignant, de même que l'instabilité du personnel scolaire. Le bilan-synthèse du portrait des parents est présenté au tableau 6.2. 
Tableau 6.2

Le bilan-synthèse du portrait des parents $(n=60)$

\begin{tabular}{lcccc}
\hline & \multicolumn{2}{c}{ Mères } & \multicolumn{2}{c}{ Pères } \\
\cline { 2 - 5 } & $N$ & $\%$ & $N$ & $\%$ \\
\cline { 2 - 5 } & 54 & 90,0 & 6 & 10,0 \\
\hline $\begin{array}{l}\text { Ont des pratiques éducatives } \\
\text { ayant un effet positif }\end{array}$ & 37 & 61,5 & 4 & 6,5 \\
\hline $\begin{array}{l}\text { Possèdent un diplôme secondaire } \\
\text { ou collégial }\end{array}$ & 37 & 61,5 & 3 & 5,0 \\
\hline Travaillent à temps plein & 33 & 55,0 & 4 & 6,5 \\
\hline $\begin{array}{l}\text { Font partie de la classe } \\
\text { moyenne ou moyenne supérieure }\end{array}$ & 32 & 53,5 & 5 & 8,5 \\
\hline Sont âgés de 25 à 34 ans & 29 & 48,5 & 1 & 1,5 \\
\hline $\begin{array}{l}\text { N'interviennent pas toujours } \\
\text { lorsqu'ils sont fatigués }\end{array}$ & 28 & 46,5 & 3 & 5,0 \\
\hline $\begin{array}{l}\text { Vivent des événements stressants } \\
\text { dans la famille }\end{array}$ & 27 & 45,0 & 2 & 3,5 \\
\hline Vivent dans un contexte de pauvreté & 22 & 36,5 & 1 & 1,5 \\
\hline $\begin{array}{l}\text { Ont des pratiques éducatives } \\
\text { ayant un effet négatif }\end{array}$ & 20 & 33,5 & 3 & 5,0 \\
\hline Interviennent trop & 14 & 23,0 & 2 & 3,5 \\
\hline N'ont pas de discipline assez stricte & 11 & 18,0 & & \\
\hline
\end{tabular}

\section{LA COLLABORATION ENTRE L'ÉCOLE ET LES PARENTS}

Selon les plus récents écrits en matière de prévention et d'intervention précoces, les parents s'avèrent des partenaires-clés pour permettre au milieu scolaire de contrer les comportements violents de leur enfant. La Fédération nationale des conseillers scolaires francophones (2003) en arrive au même constat. Toutefois, selon bon nombre d'auteurs, l'enjeu stratégique pour y parvenir semble reposer sur une bonne collaboration entre les milieux scolaire et familial. La collaboration se définit comme un cadre de travail où l'école et les parents œuvrent à l'atteinte de buts communs, à savoir contrer les comportements violents chez l'enfant. Une telle collaboration s'appuie sur une communication bidirectionnelle, enrichissante et productive, de même que sur une confiance et un soutien mutuels. Elle implique également la prise de décisions communes (Christenson et Sheridan, 2001). 
La collaboration entre l'école et les parents se distingue de l'implication parentale (Epstein, 1995) en ce qu'elle fait appel au «rôle que joue les parents dans l'éducation de leur enfant à l'école et à la maison» (Deslandes, 2001, p. 256). À ce sujet, Epstein (1995) cible six types d'implication parentale dans la vie scolaire de leur enfant, soit la participation à des activités de bénévolat à l'école, l'aide aux devoirs et aux leçons à la maison, l'appartenance à des comités de parents et à des groupes de parentage, le soutien parental à toutes autres tâches scolaires connexes et la présence de l'école dans la vie de la communauté.

En ce qui a trait aux parents de l'échantillon, un peu plus des deux tiers d'entre eux considèrent que l'école les a encouragés à s'impliquer dans au moins un des quatre premiers types. Selon Deslandes (1999), l'implication des parents dans le processus de scolarisation et de socialisation de leur enfant représente le premier pas pour mettre en place une bonne collaboration entre l'école et les parents. Il est à noter que 16 des 19 parents de l'échantillon ayant des enfants âgés de 3 à 6 ans s'impliquent sous une forme ou une autre. De plus, 25 des 33 parents qui possèdent un diplôme collégial ou universitaire sont impliqués dans la vie scolaire de leur enfant.

Un autre constat émerge des résultats de la présente recherche, à savoir que la communication est considérée comme le fondement de la collaboration. Ainsi, les trois quarts des parents de l'échantillon mentionnent avoir des contacts fréquents avec l'école au sujet de la situation-problème de leur enfant. L'agenda pour les uns, des contacts plus informels pour les autres se révèlent des moyens privilégiés de communication. Or, la teneur des propos échangés entre l'école et les parents peut engendrer des tensions, car la fréquence des contacts est généralement associée aux difficultés encourues par l'enfant (Marcon, 1999; Kohl, Lengua et McMahon, 2000 ; Reynolds, Weissberg et Kasprow, 1992). La qualité de la relation et le contenu positif des messages l'emportent ainsi sur la quantité d'échanges comme prémisses à l'établissement d'une bonne collaboration entre l'école et les parents (Adams et Christenson, 2000).

Le tiers des parents déclarent avoir éprouvé des difficultés de communication au cours de ces échanges. Pour les uns, il s'agit d'un manque d'écoute de la part du personnel scolaire. Pour d'autres, de telles difficultés de communication dégénèrent en des situations conflictuelles. Les parents qui se déclarent en conflit avec l'école associent souvent le manque d'écoute et d'ouverture du personnel scolaire à leur difficulté d'établir un processus de résolution de la situation-problème de leur enfant. Une telle difficulté peut également s'inscrire dans une délimitation de frontières 
claires entre les milieux scolaire et familial. Paradoxalement, le maintien de telles frontières est décrit comme étant la caractéristique d'une bonne collaboration (Christenson et Sheridan, 2001).

Les difficultés de communication, quelles qu'en soient la teneur et l'ampleur, semblent être d'importants obstacles à la collaboration. En observant les interactions entre les intervenants œuvrant dans le domaine de la réadaptation et les parents, Bouchard et Kalubi (2003) constatent la présence de nombreuses difficultés de communication entre ces deux milieux. Déterminer ensemble les intérêts de l'enfant, échanger sur les cadres de références, reconnaître «les peurs respectives » et "saisir la portée complémentaire des savoirs» (Bouchard et Kalubi, 2003, p. 14) s'avèrent des pistes à transférer à la problématique des enfants ayant des comportements violents au préscolaire et au primaire.

Par ailleurs, une bonne collaboration entre l'école et les parents repose sur la prise de décisions communes concernant la situation-problème de l'enfant à l'école (Christenson et Sheridan, 2001). La participation des parents aux décisions concernant leur enfant paraît être un élément crucial permettant de contrer leurs comportements violents au préscolaire et au primaire. Toutefois, ce constat reflète les perceptions des parents sans que le point de vue émanant du personnel scolaire ne vienne les nuancer. Ces perceptions représentent néanmoins des pistes éventuelles à considérer en intervention précoce.

Les résultats de la présente recherche portant sur la participation parentale au processus de prise de décisions concernant leur enfant montrent un clivage très net. Par exemple, concernant la participation des parents aux décisions, un peu plus de la moitié des parents déclarent que l'école les invite à y participer, tandis que l'autre moitié pensent, à l'inverse, que l'école ne les y invite pas. Les sujets de litiges visent généralement les interventions à caractère majeur préconisées par l'école, telles que prescrire du Ritalin ${ }^{\circledR}$ à l'enfant ou recourir à diverses formes d'exclusion socioscolaire, pour ne nommer que celles-là.

Sur la question de l'influence que les parents croient exercer à l'école, les avis sont plus partagés. Les parents croient en effet avoir de l'influence sur certains points précis, mais peu au niveau de l'ensemble de la situation-problème de leur enfant. Quant aux décisions déjà prises par l'école, le tiers des parents croient exercer une influence qui permet de les renverser alors que plus d'un autre tiers d'entre eux considèrent qu'il n'en exerce aucune. Ce clivage met en relief la complexité des échanges et des enjeux liés à la collaboration entre l'école et les parents d'enfants ayant 
des comportements violents au préscolaire et au primaire. D'un autre côté, il ouvre la porte à une nouvelle analyse par profil de parents, soit selon qu'ils collaborent ou non avec l'école.

Le profil de collaboration regroupe deux parents sur cinq de l'échantillon, alors que le profil de non-collaboration concerne le tiers d'entre eux. Ce dernier profil englobe également les parents qui se déclarent en conflit avec l'école. Le bilan-synthèse de la collaboration entre l'école et les parents est présenté au tableau 6.3.

TABLEAu 6.3

Le bilan-synthèse de la collaboration entre l'école et les parents $(n=60)$

\begin{tabular}{lcccc}
\hline & \multicolumn{2}{c}{ Mères } & \multicolumn{2}{c}{ Pères } \\
\cline { 2 - 5 } & $N$ & $\%$ & $N$ & $\%$ \\
\cline { 2 - 5 } & 54 & 90,0 & 6 & 10,0 \\
\hline $\begin{array}{l}\text { Ont des contacts fréquents } \\
\text { avec le milieu scolaire }\end{array}$ & 44 & 73,5 & 1 & 1,5 \\
\hline $\begin{array}{l}\text { S'impliquent dans la vie scolaire } \\
\text { de leur enfant }\end{array}$ & 39 & 65,0 & 4 & 6,5 \\
\hline $\begin{array}{l}\text { Participent aux décisions suite } \\
\text { à l'invitation de l'école }\end{array}$ & 28 & 46,5 & 2 & 3,5 \\
\hline $\begin{array}{l}\text { Ne sont pas invités à participer } \\
\text { aux décisions }\end{array}$ & 26 & 43,0 & 4 & 6,5 \\
\hline $\begin{array}{l}\text { Utilisent régulièrement l'agenda } \\
\text { scolaire pour communiquer }\end{array}$ & 26 & 43,5 & 1 & 1,5 \\
\hline Collaborent avec l'école & 22 & 36,5 & 3 & 5,0 \\
\hline $\begin{array}{l}\text { Ont de l'influence sur les décisions } \\
\text { déjà prises par l'école }\end{array}$ & 21 & 35,0 & 1 & 1,5 \\
\hline $\begin{array}{l}\text { N'ont pas d'influence sur les } \\
\text { décisions déjà prises par l'école }\end{array}$ & 20 & 33,5 & 2 & 3,5 \\
\hline $\begin{array}{l}\text { Éprouvent des difficultés } \\
\text { de communication }\end{array}$ & 20 & 33,5 & 2 & 3,5 \\
\hline $\begin{array}{l}\text { Éprouvent du malaise sur } \\
\text { les informations échangées }\end{array}$ & 20 & 33,5 & 1 & 1,5 \\
\hline Ne collaborent pas avec l'école & 17 & 28,5 & 2 & 3,5 \\
\hline Se déclarent en conflit avec l'école & 17 & 28,5 & 2 & 3,5 \\
\hline
\end{tabular}




\section{LA COLLABORATION SELON LE MILIEU SOCIOÉCONOMIQUE}

Sur certaines données sociodémographiques, telles que l'âge de l'enfant, le niveau de scolarité des parents et le milieu socioéconomique des familles, nous observons des différences quant à la collaboration entre les milieux scolaire et familial (Dubet et Martuccelli, 1996; Epstein, 1995; Grolnick, Benjet, Kurowski et Apostoleris, 1997; Lareau, 1987; Marcon, 1999; Pelco, Jacobson, Ries et Melka, 2000; Raffaele et Knoff, 1999). Par exemple, dans la foulée des écrits sur l'implication parentale dans la vie scolaire de l'enfant, la participation aux activités de bénévolat à l'école est davantage liée aux caractéristiques des parents qui font partie de la classe moyenne et moyenne-supérieure. Quant aux parents vivant en contexte de pauvreté, soit en grande majorité des mères de famille monoparentale, ils préfèrent s'impliquer plus directement auprès de leur enfant à la maison qu'à l'école.

Dans le même ordre d'idées, concernant la communication entre l'école et les parents, nous pouvons établir des distinctions en fonction du milieu socioéconomique. Pour le mode écrit et formel, tel que l'agenda scolaire, deux fois plus de parents regroupés dans les classes moyenne et moyenne-supérieure que de parents vivant en contexte de pauvreté l'utilisent. Ces derniers semblent privilégier des moyens plus informels pour entrer en contact avec le personnel scolaire, tels qu'effectuer une visite ou un appel téléphonique à l'école pour s'informer de leur enfant. Ils possèdent leurs propres pratiques de communication et formes d'implication parentale auprès de leur enfant à la maison, qui compensent pour leur absence à l'école.

Quant aux trois différents aspects de la participation parentale au processus de prise de décisions, les distinctions en fonction du milieu socioéconomique s'amenuisent, notamment quant aux décisions déjà prises par l'école. À ce chapitre, les parents vivant en contexte de pauvreté considèrent moins que les autres exercer une influence sur de telles décisions. Seulement le quart des parents vivant en contexte de pauvreté, comparativement aux deux cinquièmes de ceux qui sont regroupés dans les classes moyenne et moyenne-supérieure, croient exercer une telle influence.

Ce constat va dans le sens du consumérisme puisqu'une attitude de consommateurs de services s'est développée chez les parents les plus scolarisés et à plus hauts revenus (Ballion, dans Hirschhorn, 2001; de Singly, 2000; Dubet, 1997; Dubet et Martuccelli, 1996; Van Zanten et Duru-Bellat, 1999; Pourtois et Desmet, 1997; Vincent et Tomlinson, 1997). Cette attitude laisse place à une plus grande implication de la part de ces 
parents (Conseil de la famille et de l'enfance, 2000). Le consumérisme peut alors devenir une source potentielle de conflits et d'évaluation du monde scolaire (Perrenoud, 2001 ; 2002). Le bilan-synthèse de la collaboration selon le milieu socioéconomique est présenté au tableau 6.4.

TABLEAU 6.4

Le bilan-synthèse de la collaboration selon le milieu socioéconomique $(n=60)$

\begin{tabular}{|c|c|c|c|c|}
\hline \multirow[b]{3}{*}{$\begin{array}{l}\text { Parents vivant en contexte } \\
\text { de pauvreté }\end{array}$} & \multicolumn{2}{|c|}{ Mères } & \multicolumn{2}{|c|}{ Pères } \\
\hline & $N$ & $\%$ & $N$ & $\%$ \\
\hline & 22 & 96,0 & 1 & 4,0 \\
\hline $\begin{array}{l}\text { Effectuent des appels } \\
\text { téléphoniques }\end{array}$ & 8 & 35,0 & & \\
\hline Font des visites à l'école & 7 & 30,5 & 1 & 4,0 \\
\hline $\begin{array}{l}\text { Font un usage régulier } \\
\text { de l'agenda scolaire }\end{array}$ & 5 & 22,0 & & \\
\hline $\begin{array}{l}\text { Exercent une forme } \\
\text { d'influence }\end{array}$ & 5 & 22,0 & 1 & 4,0 \\
\hline \multirow[t]{3}{*}{$\begin{array}{l}\text { Participent à des activités } \\
\text { de bénévolat }\end{array}$} & 3 & 13,0 & & \\
\hline & \multicolumn{2}{|c|}{ Mères } & \multicolumn{2}{|c|}{ Pères } \\
\hline & $N$ & $\%$ & $N$ & $\%$ \\
\hline $\begin{array}{l}\text { Parents des classes moyenne } \\
\text { et supérieure }\end{array}$ & 32 & 86,5 & 5 & 13,5 \\
\hline $\begin{array}{l}\text { Font un usage régulier } \\
\text { de l'agenda scolaire }\end{array}$ & 26 & 70,0 & 1 & 3,0 \\
\hline $\begin{array}{l}\text { Exercent une forme } \\
\text { d'influence }\end{array}$ & 13 & 35,0 & 1 & 3,0 \\
\hline $\begin{array}{l}\text { Participent à des activités } \\
\text { de bénévolat }\end{array}$ & 10 & 27,0 & 1 & 3,0 \\
\hline Font des visites à l'école & 7 & 19,0 & 1 & 3,0 \\
\hline $\begin{array}{l}\text { Effectuent des appels } \\
\text { téléphoniques }\end{array}$ & 6 & 16,0 & 1 & 3,0 \\
\hline
\end{tabular}




\section{LES FACTEURS DE SOUTIEN SOUS-JACENTS À LA COLLABORATION}

En fonction du regroupement des parents qui collaborent avec l'école, il devient plus aisé de répondre à l'une des questions secondaires de la présente recherche, à savoir: Quels sont les facteurs de soutien sous-jacents à la collaboration entre l'école et les parents?

À ce sujet, l'ensemble des 25 parents faisant partie du profil de collaboration entretient une perception positive à l'endroit du personnel scolaire, en émettant au moins un commentaire favorable. Ceux-ci déclarent notamment apprécier les qualités personnelles de l'enseignant, sa disponibilité et son ouverture, de même que l'attention personnalisée qu'il accorde à leur enfant.

Une bonne communication avec le personnel scolaire est un autre important facteur de soutien permettant d'atteindre des objectifs communs. Cette communication s'articule autour d'échanges quotidiens, notamment par le biais de l'agenda scolaire. De plus, des pratiques éducatives concertées et un plan d'action où les parents assurent à la maison un suivi par rapport aux événements et aux interventions ayant eu lieu à l'école, procurent un soutien mutuel. Ces pratiques traduisent une bonne collaboration, telle que définie par Christenson et Sheridan (2001).

Parmi les autres facteurs de soutien qui caractérisent les parents de ce profil, ceux-ci reconnaissent l'expertise du personnel scolaire et font des constats importants face à leur situation familiale et à leur quotidien. Certains de ces constats se rapportent à leur enfant et à son avenir. Dans la foulée des écrits sur le processus de demande d'aide (Cantin, 1998), ils perçoivent un besoin d'agir afin de trouver une solution à court ou à moyen termes. Les parents de ce profil considèrent qu'ils subissent des inconvénients marqués par rapport à la situation-problème de leur enfant à l'école. Ils en sont affectés au quotidien, ce qui les rend plus ouverts à collaborer avec le personnel scolaire afin de trouver des solutions.

La majorité des parents de ce sous-échantillon utilisent des services d'intervention sociale, à savoir qu'ils consultent des travailleurs sociaux et des psychologues, scolaires ou privés, de même que des spécialistes de la relation d'aide œuvrant notamment dans des centres communautaires. Ces professionnels remplissent un rôle crucial en appuyant les parents et, par conséquent, leur enfant. Cet appui bénéficie également aux autres membres de la famille. En ce sens, l'intervention sociale les aide à faire face à des difficultés quotidiennes importantes. 
Chez ces parents, l'intervention précoce auprès des enfants joue un rôle très important. Plusieurs d'entre eux nomment les enjeux reconnus et associés à ce type d'intervention, notamment que les comportements violents de leur enfant peuvent être un facteur prédicteur de délinquance et de criminalité, tant à l'adolescence qu'à l'âge adulte (Farrington, 2000; Kosterman, Graham, Hawkins, Catalano et Herrenkohl, 2001; Stormont, 2000 ; Tremblay, 2000). Le bilan-synthèse des facteurs de soutien sous-jacents à la collaboration est présenté au tableau 6.5.

TABleau 6.5

Le bilan-synthèse des facteurs de soutien sous-jacents à la collaboration $(n=25)$

\begin{tabular}{lcccc}
\hline & \multicolumn{2}{c}{ Mères } & \multicolumn{2}{c}{ Pères } \\
\cline { 2 - 5 } & $N$ & $\%$ & $N$ & $\%$ \\
\cline { 2 - 5 } & 21 & 84,0 & 4 & 16,0 \\
\hline $\begin{array}{l}\text { Entretiennent une perception } \\
\text { positive de l'enseignant }\end{array}$ & 21 & 84,0 & 4 & 16,0 \\
\hline $\begin{array}{l}\text { Entretiennent une bonne } \\
\text { communication avec l'école }\end{array}$ & 21 & 84,0 & 4 & 16,0 \\
\hline $\begin{array}{l}\text { Reconnaissent l'expertise } \\
\text { du personnel scolaire }\end{array}$ & 21 & 84,0 & 4 & 16,0 \\
\hline $\begin{array}{l}\text { Reconnaissent leur } \\
\text { complémentarité }\end{array}$ & 21 & 84,0 & 2 & 8,0 \\
\hline $\begin{array}{l}\text { Utilisent des services } \\
\text { d'intervention sociale }\end{array}$ & 21 & 84,0 & 2 & 8,0 \\
\hline $\begin{array}{l}\text { Font des constats sur eux-mêmes } \\
\text { et sur leur enfant }\end{array}$ & 21 & 84,0 & 1 & 4,0 \\
\hline
\end{tabular}

\section{LES OBSTACLES SOUS-JACENTS À LA COLLABORATION}

En fonction du regroupement des parents qui ne collaborent pas avec l'école, il devient également aisé de répondre à une autre des questions secondaires de la recherche à savoir: Quels sont les obstacles sous-jacents à la collaboration entre l'école et les parents?

À ce sujet, l'ensemble des 19 parents faisant partie du profil de noncollaboration entretiennent une perception négative à l'endroit du personnel scolaire et émet au moins un commentaire défavorable. Notamment, ils disent avoir beaucoup de difficultés à accepter que le personnel scolaire 
prenne seul les décisions concernant leur enfant, sans même les consulter. Le fait d'être écarté du processus de prise de décisions semble l'élément qui alimente le plus les tensions, voire le conflit avec l'école. De plus, la grande majorité des parents de ce profil constatent que le manque de ressources et le manque de temps affectent beaucoup la situation-problème de leur enfant à l'école.

Montandon et Perrenoud (1994) avancent que la perspective d'asymétrie de pouvoir, soit la force décisionnelle de l'école, agit au détriment de celle des parents. Si ces derniers perçoivent qu'ils exercent peu ou pas d'influence, voire de contrôle sur la situation-problème de leur enfant à l'école, ils auront tendance à se retirer du processus décisionnel. Ce retrait met en relief la complexité des échanges et des enjeux liés à la collaboration entre l'école et les parents d'enfants ayant des comportements violents au préscolaire et au primaire.

Par ailleurs, les trois quarts des parents de ce sous-échantillon éprouvent un sentiment d'impuissance. Ils se disent touchés par la situation-problème de leur enfant, que ce soit par rapport à leurs émotions ou à une sensation de perte de contrôle sur leur réalité (Le Bossé, 1996; Rappaport, 1987).

D'autres obstacles sous-jacents sont considérés comme d'importants freins à la collaboration entre les milieux scolaire et familial. À cet effet, certains parents du profil de non-collaboration jugent parfois recevoir des jugements et des qualificatifs lourds de sens, voient un désengagement de la part de l'école à l'égard de leur enfant et sentent une volonté de l'institution de l'exclure du processus de scolarisation et de socialisation. Ces enjeux rejoignent, en très grande majorité, les parents d'enfants de 6 ans et moins, regroupés dans les classes moyenne et moyenne-supérieure.

Ce constat conduit à nous interroger au sujet des retombées de l'exclusion socioscolaire des enfants d'âge préscolaire ayant des comportements violents. Face à la lourdeur de cette problématique et aux ressources d'intervention qu'elle requiert, la collaboration entre l'école et les parents d'un jeune enfant s'avère d'autant plus nécessaire (Deslandes, 2001 ; Eccles et Harold, 1991; Epstein, 1995; Organisation de coopération et de développement économiques, 1997; Montandon, 1996). L'implication accrue des parents en raison du jeune âge de l'enfant explique ainsi leur perspective davantage critique face aux décisions que prend l'école sans les consulter. 
D'après d'autres parents, les stéréotypes auxquels le personnel scolaire recourt et qui concernent les facteurs de risque de la violence représentent un autre obstacle important à la collaboration. Selon eux, le personnel scolaire juge un peu trop facilement les parents et n'hésite pas à utiliser ces stéréotypes comme prétexte pour définir la situation-problème, voire pour diminuer leur contribution parentale. Par exemple, l'âge de la mère à la naissance de l'enfant concerné ou du premier enfant, son niveau de scolarité, son statut marital, son groupe ethnoculturel, l'absence du père quelle qu'en soit la cause, ne sont que quelques-uns des motifs sur lesquels le milieu scolaire base son jugement à son endroit.

Afin de se protéger, voire de survivre dans un tel contexte, ces mères n'hésitent pas à leur tour à recourir à de tels stéréotypes. Par exemple, elles invoquent facilement le jeune âge de l'enseignant, son manque d'expérience, le fait qu'il ne soit pas un parent, pour le discréditer ou amoindrir l'effet de son étiquetage. Le recours à de tels stéréotypes par les parents vise également la direction d'école, qui est la personne-clé associée à l'institution, à ses procédures, à son pouvoir décisionnel ainsi qu'à l'accès aux ressources et aux services pouvant être mis à la disposition de leur enfant, afin de lui venir en aide.

Or, selon Christenson et Sheridan (2001), le blâme mutuel participe activement au litige. À cet effet, autant le parent a peur d'être montré du doigt lors de difficultés (Dubet et Martuccelli, 1996), autant les enseignants développent une réaction de défense face à l'éventualité que l'on remette en question leur pratique professionnelle. Tous ces éléments contribuent à maintenir une distance, voire un fossé entre eux (Cifali, 1997; Montandon, 1994). Pour surmonter ces obstacles, il importe de dépasser l'individualisation des enjeux afin de pouvoir mieux les contextualiser (Montandon et Perrenoud, 1994). Le bilan-synthèse des obstacles sous-jacents à la collaboration est présenté au tableau 6.6. 
TABLEAU 6.6

Le bilan-synthèse des obstacles

sous-jacents à la collaboration $(n=19)$

\begin{tabular}{lcccc}
\hline & \multicolumn{2}{c}{ Mères } & \multicolumn{2}{c}{ Pères } \\
\cline { 2 - 5 } & $N$ & $\%$ & 2 & $\%$ \\
\cline { 2 - 5 } & 17 & 89,5 & 2 & 10,5 \\
\hline $\begin{array}{l}\text { Entretiennent une perception } \\
\text { négative du personnel scolaire }\end{array}$ & 17 & 89,5 & 2 & 10,5 \\
\hline $\begin{array}{l}\text { Ne sont pas impliqués dans } \\
\text { les décisions }\end{array}$ & 17 & 89,5 & 2 & 10,5 \\
\hline $\begin{array}{l}\text { Ne sont pas consultés par le } \\
\text { personnel scolaire }\end{array}$ & 17 & 89,5 & 2 & 10,5 \\
\hline $\begin{array}{l}\text { Constatent un manque de } \\
\text { ressources et de temps }\end{array}$ & 17 & 89,5 & 1 & 5,5 \\
\hline $\begin{array}{l}\text { Éprouvent un sentiment } \\
\text { d'impuissance }\end{array}$ & 13 & 68,5 & & 5,5 \\
\hline $\begin{array}{l}\text { Reçoivent des qualificatifs et } \\
\text { jugements lourds de sens }\end{array}$ & 12 & 63,0 & 1 & 10,5 \\
\hline $\begin{array}{l}\text { Concernent les facteurs de risque } \\
\text { de la violence }\end{array}$ & 11 & 58,0 & 2 & 5,5 \\
\hline $\begin{array}{l}\text { Sentent une volonté de l'école } \\
\text { d'exclure leur enfant }\end{array}$ & 10 & 52,5 & 1 & \\
\hline $\begin{array}{l}\text { Perçoivent un désengagement } \\
\text { face à leur enfant }\end{array}$ & 10 & 52,5 & & \\
\hline $\begin{array}{l}\text { Recourent à leur tour à des } \\
\text { stéréotypes }\end{array}$ & 5 & 26,5 & & \\
\hline
\end{tabular}

\section{LES GESTES D'EMPOWERMENT POSÉS PAR LES PARENTS}

Un autre aspect du questionnement de la présente recherche porte sur l'empowerment parental. La troisième question secondaire de recherche se formule de la manière suivante: Quels sont les gestes d'empowerment posés par les parents leur permettant d'exercer une influence, voire un contrôle, sur la situation-problème de leur enfant à l'école?

L'empowerment se définit comme le sentiment ou la perception d'exercer une influence, voire un contrôle sur les événements de sa vie (Rappaport, 1987). Les gestes posés par les parents qui découlent de 
ce sentiment ou de cette perception peuvent être de plusieurs types. Notamment, il peut s'agir de gestes d'adaptation et d'opposition qui visent à influencer positivement la situation-problème de l'enfant, ainsi qu'à mobiliser des ressources à mettre à sa disposition afin de lui venir en aide. Il peut également s'agir de gestes de résistance, qui consistent en des actes intentionnels ou des omissions volontaires de la part des parents, visant à défier les désirs des membres du personnel scolaire (Ashforth et Mael, 1998). Les gestes d'empowerment peuvent également consister en des pratiques silencieuses de protestation.

Quatre parents sur cinq posent au moins un geste d'empowerment en vue d'exercer une influence, voire un contrôle sur la situation-problème de leur enfant à l'école. Ces gestes consistent principalement à amorcer la résolution de la situation-problème, à s'opposer aux interventions majeures de l'école, à convoquer une rencontre avec le personnel scolaire, à proposer des solutions, à consulter un tiers à l'extérieur à l'école, à discuter des comportements ou assurer un suivi, à se conformer aux demandes de l'école, à se taire et adopter la position de l'école et à changer l'enfant d'école ou de garderie.

Par rapport à certaines variables sociodémographiques relatives à l'enfant ou à sa famille, aucune analyse ne révèle de distinctions quant aux gestes d'empowerment posés par les parents. Toutefois, si la très grande majorité des parents s'avèrent actifs et impliqués, il importe de souligner que ce sont les parents d'enfants âgés de 6 ans et moins qui le sont le plus. Ces derniers posent au moins cinq gestes d'empowerment différents. Le jeune âge des enfants favorise l'implication accrue de ces parents, en suscitant une plus grande compréhension critique de sa situation-problème. Dans la foulée des écrits portant sur la communication, les parents qui posent de nombreux gestes d'empowerment peuvent indiquer qu'ils éprouvent de grandes difficultés et tensions avec l'école. Par conséquent, ce n'est pas la quantité de gestes posés qui importe, mais le contexte dans lequel ils s'inscrivent. Par exemple, dans un contexte de collaboration, un geste posé par un parent peut contribuer à résoudre la situation-problème alors que, dans un contexte de non-collaboration, le même geste risque de la faire dégénérer en relation conflictuelle avec l'école.

En définitive, pour les parents qui se perçoivent comme des acteurs à part entière, la collaboration avec l'école leur procure des occasions de poser des gestes d'empowerment. Le bilan-synthèse des gestes d'empowerment posés par les parents est présenté au tableau 6.7. 
TABleau 6.7

Le bilan-synthèse des gestes d'empowerment posés par les parents $(n=48)$

\begin{tabular}{lcccc}
\hline & \multicolumn{2}{c}{ Mères } & \multicolumn{2}{c}{ Pères } \\
\cline { 2 - 5 } & $N$ & $\%$ & $N$ & $\%$ \\
\cline { 2 - 5 } & 44 & 91,5 & 4 & 8,5 \\
\hline $\begin{array}{l}\text { Amorcent la résolution de la } \\
\text { situation-problème }\end{array}$ & 20 & 41,5 & 1 & 2,0 \\
\hline $\begin{array}{l}\text { S'opposent aux interventions } \\
\text { majeures de l'école }\end{array}$ & 19 & 39,5 & 1 & 2,0 \\
\hline $\begin{array}{l}\text { Convoquent une rencontre avec } \\
\text { le personnel scolaire }\end{array}$ & 17 & 35,5 & 2 & 4,0 \\
\hline $\begin{array}{l}\text { Proposent des solutions } \\
\text { Consultent un tiers à l'extérieur }\end{array}$ & 15 & 31,5 & 1 & 2,0 \\
\hline de l'école & 14 & 29,0 & 1 & 2,0 \\
\hline $\begin{array}{l}\text { Discutent des comportements } \\
\text { ou assurent un suivi }\end{array}$ & 13 & 27,0 & 1 & 2,0 \\
\hline $\begin{array}{l}\text { Se conforment aux demandes } \\
\text { de l'école }\end{array}$ & 13 & 27,0 & 1 & 2,0 \\
\hline $\begin{array}{l}\text { Se taisent et adoptent la position } \\
\text { de l'école }\end{array}$ & 13 & 27,0 & 1 & 2,0 \\
\hline $\begin{array}{l}\text { Changent l'enfant d'école ou } \\
\text { de garderie }\end{array}$ & 11 & 23,0 & 2 & 4,0 \\
\hline
\end{tabular}




\section{LES PERCEPTIONS DES PARENTS FACE À LA SUSPENSION SCOLAIRE EXTERNE}

Au sujet de la suspension scolaire externe, les deux tiers des parents de l'échantillon ne semblent pas se rallier à l'idée qu'elle soit une mesure disciplinaire appropriée à l'enfant de 3 à 9 ans. D'une part, les parents croient qu'elle n'améliore pas les comportements violents, étant même contraire au but recherché, car elle encourage la récidive chez l'enfant. D'autre part, ils ne constatent aucun effet positif suite à son application à répétition. Aussi, les parents déclarent que, loin de régler la situation-problème à la source, la suspension scolaire externe paraît même agréable aux yeux de leur enfant, pour qui elle représente une occasion de se trouver en milieu familial avec eux.

Par ailleurs, le tiers des parents de l'échantillon considèrent que cette mesure disciplinaire est très dommageable, au point de vue tant de la scolarisation que de la socialisation de leur enfant. Ces propos rejoignent les conclusions des études menées par Fasko, Grubb et Osborne (1997), Hayden et Ward (1996) et Royer (1995). De plus, ils la considèrent inefficace chez les enfants en bas âge et craignent fort qu'elle soit un facteur d'exclusion socioscolaire. Ces résultats se situent dans le prolongement d'études qui concluaient à des appréhensions similaires chez les parents (Hayden, 1994; Blyth and Milner, 1993; Royer, Bitaudeau et Saint-Laurent, 1993).

Le quart des parents pensent que l'application de cette mesure est injustifiée pour des motifs banals, car elle perd alors toute crédibilité aux yeux de l'enfant. Selon eux, elle ne devrait être appliquée que de manière graduelle, et ce, lorsque la gravité de la situation-problème l'exige. Dans tous les cas, les parents expriment le souhait d'être consultés avant que l'école ne décide de son application. De plus, ils veulent être partie prenante dans la décision de l'appliquer ou non. Ces propos rejoignent ceux de l'étude de Dupper (1994), qui conclut que la suspension scolaire externe contribue à générer un contexte conflictuel entre l'école et les parents, surtout lorsque ces derniers ne sont pas consultés avant son application ou qu'ils s'y opposent une fois la décision déjà prise par l'école. Le bilansynthèse des perceptions des parents face à la suspension scolaire externe est présenté au tableau 6.8. 
TABleau 6.8

Le bilan-synthèse des perceptions des parents

face à la suspension scolaire externe $(n=60)$

\begin{tabular}{lcccc}
\hline & \multicolumn{2}{c}{ Mères } & \multicolumn{2}{c}{ Pères } \\
\cline { 2 - 5 } & $N$ & $\%$ & $N$ & $\%$ \\
\cline { 2 - 5 } & 54 & 90,0 & 6 & 10,0 \\
\hline $\begin{array}{l}\text { Croient qu'elle n'est pas } \\
\text { appropriée à leur enfant }\end{array}$ & 34 & 56,5 & 5 & 8,5 \\
\hline $\begin{array}{l}\text { Croient qu'elle n'améliore pas } \\
\text { les comportements }\end{array}$ & 34 & 56,5 & 5 & 8,5 \\
\hline $\begin{array}{l}\text { Souhaitent être consultés avant } \\
\text { son application }\end{array}$ & 32 & 53,5 & 4 & 6,5 \\
\hline $\begin{array}{l}\text { Ne croient pas à son efficacité } \\
\text { en bas âge }\end{array}$ & 29 & 48,5 & 3 & 5,0 \\
\hline $\begin{array}{l}\text { Croient qu'elle ne règle pas la } \\
\text { situation-problème }\end{array}$ & 29 & 48,5 & 3 & 5,0 \\
\hline $\begin{array}{l}\text { Croient que l'application à } \\
\text { répétition est inefficace }\end{array}$ & 23 & 38,5 & 3 & 5,0 \\
\hline $\begin{array}{l}\text { Croient qu'elle s'avère } \\
\text { dommageable à l'apprentissage }\end{array}$ & 18 & 30,0 & 3 & 5,0 \\
\hline $\begin{array}{l}\text { Pensent qu'elle paraît agréable } \\
\text { aux yeux de l'enfant }\end{array}$ & 15 & 25,0 & 1 & 1,5 \\
\hline $\begin{array}{l}\text { Souhaitent être partie prenante } \\
\text { dans les décisions }\end{array}$ & 13 & 21,5 & 1 & 1,5 \\
\hline $\begin{array}{l}\text { Pensent qu'elle est injustifiée } \\
\text { pour des motifs banals }\end{array}$ & 12 & 20,0 & 2 & 3,5 \\
\hline $\begin{array}{l}\text { Considèrent qu'elle encourage } \\
\text { la récidive chez l'enfant }\end{array}$ & 11 & 18,5 & 1 & 1,5 \\
\hline
\end{tabular}

\section{LES PERCEPTIONS DES PARENTS FACE AUX AUTRES MESURES DISCIPLINAIRES}

Pour les autres mesures disciplinaires, neuf parents sur dix de l'échantillon semblent privilégier la suspension interne (Stage, 1997; Farner, 1996; Guindon, 1992 ; Sullivan, 1989). Toutefois, selon le quart d'entre eux, pour être efficace, elle devrait non pas être appliquée seule, mais s'accompagner d'activités de rattrapage scolaire et d'activités de prise de conscience (Vanderslice, 1999). 
Par ailleurs, plus des deux tiers des parents estiment que l'école devrait tenter de déterminer, cas par cas, le moyen le plus efficace de contrer les comportements violents de leur enfant, tout en adaptant les conséquences à l'acte selon son tempérament et sa personnalité. Â ce titre, un peu plus de la moitié des parents croient que l'école devrait privilégier le recours à des mesures qui semblent bien fonctionner avec leur enfant. Finalement, un peu plus du tiers des parents estiment que l'école aurait avantage à transposer certaines mesures disciplinaires qu'ils appliquent à la maison et qui donnent de bons résultats avec leur enfant.

Face à cette situation, il y a tout lieu pour l'école d'évaluer dans quelle mesure la suspension scolaire externe représente la meilleure solution pour permettre à l'élève de contrer ses comportements violents envers ses pairs au préscolaire et au primaire. Si on privilégie cette mesure disciplinaire, elle devrait s'accompagner d'un dispositif permettant aux parents de jouer adéquatement leur rôle.

En ce sens, le milieu scolaire ne doit jamais perdre de vue que toute conséquence à l'acte faisant l'objet d'un transfert en milieu familial n'a d'impact éducatif que si on sauvegarde la valeur de formation qui lui est sous-jacente (Van Neste, 1994). Si l'école privilégie la suspension interne, elle ne doit pas oublier que les conséquences ne sont pas moins graves de maintenir l'enfant violent en milieu scolaire si aucun encadrement ne lui est offert (Hartman et Stage, 2000) puisque celui-ci risque d'être retardé sur le plan des apprentissages (Feucht, 1998), en plus de faire possiblement l'objet de harcèlement et de rejet de la part de ses pairs (Blyth et Milner, 1993).

Par ailleurs, en amont de l'application de telles mesures disciplinaires, il existe des programmes de prévention des comportements violents axés sur des stratégies de résolution pacifique de conflits (Garibaldi, Blanchard et Brooks, 1996; Turcotte, Lamonde et Lindsay, 2002), ainsi que des programmes de développement des compétences sociales (Bélanger, Bowen et Rondeau, 1999; Bowen, Rondeau, Rajotte et Bélanger, 2000; Couture, 2001; Royer, Morand, Desbiens, Moisan et Bitaudeau, 2000; Saint-Laurent, Giasson, Simard, Dionne et Royer, 1995). L'argument justifiant le foisonnement de ces nouveaux programmes, depuis les années 1990, se fonde sur le fait que les élèves ayant de tels comportements envers leurs pairs au préscolaire et au primaire ne possèdent vraisemblablement pas les compétences sociales requises (Masten et Coatsworth, 1998) pour évoluer harmonieusement en milieu scolaire. L'école aurait ainsi tout avantage à accroître l'utilisation de tels programmes puisque ceux-ci semblent donner des résultats prometteurs. Le bilan-synthèse des perceptions des parents face aux autres mesures disciplinaires est présenté au tableau 6.9. 
TABleau 6.9

Le bilan-synthèse des perceptions des parents face aux autres mesures disciplinaires $(n=60)$

\begin{tabular}{lcccc}
\hline & \multicolumn{2}{c}{ Mères } & \multicolumn{2}{c}{ Pères } \\
\cline { 2 - 5 } & $N$ & $\%$ & $N$ & $\%$ \\
\cline { 2 - 5 } & 54 & 90,0 & 6 & 10,0 \\
\hline $\begin{array}{l}\text { Privilégient la suspension } \\
\text { scolaire interne }\end{array}$ & 49 & 81,5 & 3 & 5,0 \\
\hline $\begin{array}{l}\text { Estiment qu'il faut déterminer } \\
\text { la mesure la plus efficace }\end{array}$ & 37 & 61,5 & 4 & 6,5 \\
\hline $\begin{array}{l}\text { Suggèrent que l'école utilise } \\
\text { des mesures qui fonctionnent }\end{array}$ & 30 & 50,0 & 3 & 5,0 \\
\hline $\begin{array}{l}\text { Croient que l'école devrait } \\
\text { transposer certaines mesures qui } \\
\text { fonctionnent bien en milieu familial }\end{array}$ & 19 & 31,5 & 2 & 3,5 \\
\hline $\begin{array}{l}\text { Croient qu'elles doivent } \\
\text { s'accompagner d'activités } \\
\text { de rattrapage scolaire }\end{array}$ & 15 & 25,0 & 2 & 3,5 \\
\hline $\begin{array}{l}\text { Croient qu'elles doivent } \\
\text { inclure des activités } \\
\text { de prises de conscience }\end{array}$ & 13 & 21,5 & 2 & 3,5 \\
\hline
\end{tabular}

\section{LES ÉVENTUELLES PISTES D'INTERVENTION ET DE RECHERCHE}

Cette recherche a permis aux parents d'exprimer leurs perceptions au sujet de la situation-problème de leur enfant à l'école. Notamment, tout au long des entrevues, ils ont pu exprimer leurs besoins sous différentes formes. Nous traduisons ces besoins en pistes éventuelles pour l'intervention et la recherche.

Comme première piste $d$ 'intervention, tant les parents du profil de collaboration que ceux du profil de non-collaboration reconnaissent le besoin de pouvoir compter sur une personne-ressource (travailleur social, psychologue scolaire, animateur de pastorale, etc.) qui leur fournisse un soutien et un appui indispensables lors d'échanges avec l'école. Puisque le geste d'empowerment qui semble valoriser le plus le rôle du parent s'avère être la contribution d'un tiers, une telle ressource ne pourrait que mieux faciliter sa communication avec l'école: Il nous faudrait quelqu'un comme un médiateur. Quelqu'un qui pourrait donner de la formation. Un système d'appui pour les parents. Comme une personne qui expliquerait que c'est ça le processus 
(108). La personne-ressource devient alors un pont permettant de donner la parole à chaque partie. Elle permet également de reconnaître tant les apports et les savoirs de l'école que ceux des parents, le tout au bénéfice de l'enfant. Cette recommandation va également dans le sens des conclusions de Bouchard et Kalubi (2003).

Une deuxième piste d'intervention a trait à la mise en place d'un réseau de soutien informel. À ce sujet, au moment où la souffrance est palpable, lorsque les parents éprouvent des difficultés à tous les plans, que les enfants ont les comportements les plus violents et que la relation avec l'école dégénère en conflit, les parents réclament un soutien inconditionnel de la part de leur réseau informel. Ce soutien agit à plusieurs niveaux. Pour les uns, il s'agit de briser l'isolement social, de dédramatiser la situationproblème, d'avoir des trucs de "parenting" pour passer à travers et finir par avoir le dessus (108). Pour d'autres, il leur permet d'être rassurés tout en ne faisant pas l'objet de jugements de valeurs ou préjugés: Je pense que le plus gros, c'est que tu peux conter tes histoires. Dire ce qu'il a fait. Juste pour te vider. Puis tu sais que l'autre dira pas que ça n'a pas d'allure! (111). Ce soutien peut provenir du voisinage, de la parenté, des parents d'autres enfants affrontant les mêmes embûches: X est le meilleur ami de mon fils. Donc, on a décidé de faire des activités avec ses parents, de temps en temps. Comme aller au musée, ou faire un souper chez nous ou chez eux... C'est très très positif et je trouve vraiment bon de pouvoir m'asseoir et de parler avec eux (108). Bien qu'un réseau existant permet de maximiser les facteurs de soutien déjà en action (Daumas-Saab, 2001), d'autres moyens peuvent également être mis à contribution, sous la forme d'un forum de discussion sur Internet ou même d'un groupe de parentage, organisé ou non par l'école.

Enfin, dans un esprit de formation, une troisième piste d'intervention concerne la mise en œuvre et l'évaluation d'un programme de suspension scolaire interne. À cet effet, un tel programme, comprenant des activités de rattrapage scolaire et des activités de conscientisation, ne pourrait que mieux permettre à l'enfant de surmonter sa situation-problème à l'école. Dans cet esprit, le fait de suspendre l'enfant pour motif de comportements violents envers ses pairs au préscolaire et au primaire devrait lui permettre de vivre différemment son processus de scolarisation et de socialisation plutôt que d'en interrompre le cours.

Quant aux pistes de recherche, elles concernent les nouvelles réalités familiales. Une première piste porte sur l'étude de la transformation des conditions de travail des parents (Higgins et Duxbury, 2002), afin de mieux connaître et comprendre leurs impacts sur les conditions de vie des enfants, leur quotidien, l'éducation, la santé, etc. À ce sujet, la conciliation travailfamille semble constituer un des thèmes dominants dans la recherche menée actuellement en sciences humaines et sociales. 
Une deuxième piste de recherche concerne l'importance de mieux connaître et comprendre la valeur que le personnel scolaire (direction d'école, enseignants et personnel non enseignant, tels que les travailleurs sociaux scolaires, les psychologues scolaires, les enseignants-ressources, etc.) accorde aux pratiques d'inclusion socioscolaire, aux mécanismes d'insertion à l'environnement socioscolaire, ainsi qu'aux mesures d'intégration et de réinsertion faisant suite à toutes formes d'exclusion socioscolaire de l'enfant. Une telle étude permettrait d'analyser les conditions de la pratique enseignante ayant cours non seulement à l'éducation préscolaire, mais également au primaire puisque l'entrée en première année constitue une transition importante qui, si elle n'est pas réussie, peut entraîner des effets qui perdurent au-delà des premières années de scolarisation. Selon Deslandes et Jacques (2004), cette transition est perçue comme une période de vulnérabilité chez l'enfant au cours de laquelle il développe des attitudes, des comportements et des compétences qui ont un rôle capital dans son développement et sa réussite socioscolaires futurs.

Par ailleurs, les comportements anxieux et dépressifs ainsi que la souffrance morale observés chez les enfants de l'échantillon nous amènent à penser qu'ils jouent un rôle crucial dans la production de violence au préscolaire et au primaire. Ces constats rejoignent les propos de Hayden et Ward (1996) qui concluent à l'importance d'intervenir plus efficacement auprès des enfants ayant des problèmes internalisés. À cet égard, le manque de ressources humaines spécialisées œuvrant au préscolaire et au primaire, ainsi que le foisonnement de nouvelles lois et réglementations concernant la sécurité dans les écoles, font en sorte que le milieu scolaire ne s'attarde qu'aux gestes observables de la violence, soit sur le plan des problèmes externalisés. De plus, les constats portant sur les compétences éducatives des parents et l'acquisition des compétences sociales chez les enfants occultent l'analyse de leur situation-problème, en raison de l'importance accordée aux conditions de vie familiale. Face à cette problématique, une troisième piste de recherche concerne l'importance de mieux connaître et comprendre l'effet des comportements internalisés de l'enfant sur la production de violence au préscolaire et au primaire afin, éventuellement, de mieux pouvoir agir sur cette problématique.

En terminant, tous les projets d'intervention et de recherche menés dans le champ de la prévention de la violence au préscolaire et au primaire ne pourront que contribuer au mieux-être des enfants, de leur famille, de l'école, de la communauté, voire de toute une société. 
Conclusion 

Dans cet ouvrage basé sur les résultats d'une recherche, nous avons exposé les perceptions des parents d'enfants de 3 à 9 ans ayant des comportements violents envers leurs pairs au préscolaire et au primaire. D'une part, cette recherche visait à mieux connaître et comprendre la teneur de leurs comportements et les facteurs explicatifs de leur situation-problème. D'autre part, elle s'est intéressée à la collaboration entre l'école et les parents, soit aux facteurs de soutien ou aux obstacles sous-jacents, de même qu'aux gestes d'empowerment posés par les parents afin d'exercer une influence, voire un contrôle sur la situation-problème de leur enfant à l'école. Finalement, elle visait à recueillir les perceptions parentales face à la suspension scolaire et aux autres mesures disciplinaires appliquées par l'école en réponse aux comportements violents de leur enfant, et ce, en relation avec les récentes législations fédérales et provinciales encadrant la violence en milieu scolaire.

Pour faire partie de l'échantillon, les enfants devaient avoir fait preuve de comportements violents envers leurs pairs au préscolaire et au primaire et avoir été l'objet d'une suspension ou d'une possibilité de suspension scolaire, au sens de la Loi sur la sécurité dans les écoles (Ministère de l'Éducation de l'Ontario, 2000) et du Code de conduite provincial (Ministère de l'Éducation de l'Ontario, 2001a). L'échantillon a été constitué de volontaires ayant accepté de réaliser une entrevue téléphonique. Le recrutement des parents s'est fait auprès des deux conseils scolaires de langue française, dans les médias et auprès d'organismes communautaires francophones œuvrant dans la région d'Ottawa, soit le Centre psychosocial pour enfants et familles et le Centre de ressources de la Basse-ville. Ces entrevues téléphoniques ont duré de trente minutes à deux heures. La moitié d'entre elles étaient structurées et l'autre moitié, semi-structurées, le tout en fonction de la disponibilité des parents. Elles ont été menées entre juin 2000 et juin 2002.

L'analyse des données d'entrevues visait à répondre aux questions de recherche, principale et secondaires, auxquelles nous nous sommes intéressées, à savoir: Quels sont les défis et les enjeux de la collaboration entre l'école et les parents d'enfants de 3 et 9 ans afin de contrer leurs comportements violents envers leurs pairs au préscolaire et au primaire? Quel est le portrait des enfants de 3 à 9 ans ayant des comportements violents envers leurs pairs à l'école et de leurs parents? Quels sont les éléments constituant un contexte de collaboration et de non-collaboration entre l'école et les parents? Quels sont les facteurs de soutien et les obstacles sous-jacents? Quels sont les gestes d'empowerment posés par les parents leur permettant d'exercer une influence, voire un contrôle sur la situationproblème de leur enfant à l'école? Quelles sont les mesures disciplinaires 
appliquées par l'école permettant de contrer les comportements violents des 3 à 9 ans et de ne pas entraver leur processus de scolarisation et de socialisation?

Afin d'apporter des pistes de réponses à ces questions, nous avons consacré le premier chapitre de cet ouvrage à la mise en contexte de l'étude. Ce chapitre a débuté avec la problématique des enfants ayant des comportements violents envers leurs pairs au préscolaire et au primaire. Au départ, nous avons exposé la complexité de cette problématique et les facteurs de risque, suivis du cadre légal relatif au contrôle de la violence dans les écoles, notamment celui qui est propre au milieu scolaire de langue française en Ontario.

Quant au deuxième chapitre, nous l'avons consacré à la collaboration entre l'école et les parents. Nous avons établi une distinction entre les concepts d'implication et de collaboration parentales. Par la suite, nous avons circonscrit les facteurs de soutien et les obstacles sous-jacents à la collaboration. Puis, nous avons présenté la définition d'un autre concept-clé, soit celui d'empowerment, qui génère des gestes d'adaptation, d'opposition et de résistance posés par les parents afin d'exercer une influence, voire un contrôle sur la situation-problème de leur enfant à l'école.

Le troisième chapitre a présenté le portrait des enfants violents et celui de leurs parents. Nous avons également décrit la méthodologie de recherche. Nous avons présenté l'analyse qualitative des données selon le regroupement des enfants de 3 à 6 ans et de leurs parents, puis selon celui des enfants de 6 à 9 ans et de leurs parents. Ces regroupements tiennent compte des deux importantes transitions qui surviennent dans la vie de l'enfant au cours de cette période, soit celle entre la maison/garderie et le préscolaire, puis celle entre le préscolaire et le primaire. L'analyse des données a fait notamment ressortir les perceptions parentales face aux comportements violents de leur enfant, leurs pratiques éducatives, les stresseurs familiaux qu'ils affrontent, ainsi que les facteurs explicatifs de la situation-problème de leur enfant à l'école.

Quant au quatrième chapitre de l'ouvrage, nous l'avons entièrement consacré à l'analyse des données portant sur la collaboration entre l'école et les parents. Nous avons examiné les perceptions des parents dans le but de mieux cerner les trois concepts sous-jacents, soit l'implication parentale, la communication entre les deux milieux ainsi que le processus de prise de décisions communes concernant l'enfant. Nous avons circonscrit certains facteurs de soutien et obstacles sous-jacents à cette collaboration ainsi que les gestes d'empowerment posés par les parents. Nous avons présenté certains résultats en fonction du milieu socioéconomique des familles, ainsi 
qu'en fonction de l'âge de l'enfant et du niveau de scolarité des parents, puisque ces variables s'avèrent de précieux indicateurs de la collaboration entre les milieux scolaire et familial.

Le cinquième chapitre a été consacré à la suspension scolaire, qui représente un enjeu crucial lié à la problématique des enfants ayant des comportements violents envers leurs pairs au préscolaire et au primaire. Dans ce chapitre, nous avons notamment fait le point sur les perceptions que les parents entretiennent face à l'application de cette mesure disciplinaire en réponse à de tels comportements. Après avoir exposé les principaux constats, nous avons présenté une alternative à la suspension scolaire externe, soit la suspension interne, de même que des suggestions d'application de mesures disciplinaires adaptées à chaque situationproblème, le tout dans l'optique que les parents soient des collaborateurs, voire des partenaires à part entière dans le processus de prise de décisions concernant leur enfant.

Enfin, dans le sixième chapitre, nous avons dressé le bilan-synthèse des résultats de la recherche, dont les principales caractéristiques des enfants et de leurs parents. Par la suite, nous avons enchaîné avec les principaux constats qui se dégagent de l'analyse des données au sujet de la collaboration entre l'école et les parents, des facteurs de soutien et des obstacles sous-jacents à cette collaboration, des gestes d'empowerment posés par les parents, ainsi que de leur perception de la suspension scolaire comme mesure disciplinaire appliquée en réponse aux comportements violents de leur enfant envers leurs pairs au préscolaire et au primaire. Pour terminer, nous avons proposé des pistes éventuelles d'intervention et de recherches.

Face à l'importance de la problématique de la violence au préscolaire et au primaire et au regard des principaux constats qui se dégagent de la présente recherche, il importe que toutes les instances soient mises à contribution, à savoir: les parents, les enfants, l'école, les services sociaux et le milieu communautaire. Sans véritable collaboration, voire partenariat entre ces instances, toutes tentatives pour contrer les comportements violents des enfants au préscolaire et au primaire sont vouées à l'échec.

Bref, les préoccupations des parents d'enfants ayant des comportements violents au préscolaire et au primaire nous interpellent en raison de leur détresse. Leurs propos sont dérangeants. Accepter d'être à leur écoute afin qu'ils puissent exprimer leurs besoins n'est pas chose facile. À ce titre, s'assurer de la collaboration indéfectible des parents représente un défi de taille pour l'école. Cette collaboration repose sur des enjeux qui dépassent largement le cadre de la bonne entente. Toutefois, le fait de donner suite 
aux propos des parents, constamment en quête de solutions, ne peut que contribuer au mieux-être des enfants, de leur famille, de l'école, de la communauté, voire de toute la société.

En définitive, la collaboration entre l'école et les parents est une préoccupation relativement nouvelle, même si ses modalités ont toujours fait l'objet de nombreux questionnements. Dans ce contexe, il y a fort à parier que les prochaines années donneront lieu à des transformations importantes dans leur rapport, puisque ces concepts tendent à être dorénavant inclus au contenu de programmes de prévention, d'intervention, de recherche et de formation. Nous espérons que cet ouvrage contribuera positivement à cette tendance. 
Bibliographie 

Adams, K.S. et S.L. Christenson (2000). «Trust and the family-school relationship examination of parent-teacher differences in elementary and secondary grades », Journal of School Psychology, 38(5), p. 477-497.

Adams, R. (1996). Social Work and Empowerment, Londres, Macmillan.

Akande, A. (2001). «A way of being: A program for aggression control of male children », Early Child Development and Care, 167, p. 127-148.

Akkari, A. (1999). «Participation des parents au processus de scolarisation: recherches, modèles d'action et débats théoriques", Journal of Educational Thought, 33(3), p. 231-253.

Algozzine, B. et P. Kay (2002). Preventing Problem Behaviors: A Handbook of Successful Prevention Strategies, Thousands Oaks, Corwin.

American Psychiatric Association (1996). The Diagnostic and Statistical Manual of Mental Disorders, 4 éd., MINI DSM-IV, Critères diagnostiques (Washington, 1994), traduction française par J.-D. Guelfi, Paris, Masson.

Anatrella, T. (1995). «Les enfants violents à l'école primaire: qui sont-ils et que deviennent-ils?», dans Y. L'Abbé (dir.), Violence chez les jeunes, Montréal, Sciences et Culture, p. 37-49.

Angelino, I. et C. Meyer (2001). La prévention des difficultés éducatives et sociales, Paris, Dunod.

Armstrong, J., J. Caron et M. Guimond (1992). Programme de formation de parents d'enfants désobéissants, Montréal, Arcamon.

Ashforth, B.E. et F.A. Mael (1998). «The power of resistance: Sustaining valued identities», dans R.M. Kramer et M.A. Neale (dir.), Power and Influence in Organizations, Thousand Oaks, Sage, p. 89-119.

Astor, R.A. (1995). «School violence: A blueprint for elementary school interventions», School Work in Education, 17(2), p. 101-115.

Baudier, A. et B. Céleste (2000). Le développement affectif et social du jeune enfant: un exposé clair et précis qui rend compte de la complémentarité des approches théoriques, Paris, Nathan.

Baumrind, D. (1991). «Parenting styles and adolescent development», dans R.M. Lerner, A.C. Petersen et J. Brooks-Gunn (dir.), Encyclopedia of Adolescence, New York, Garland, 2, p. 726-758.

Bélanger, J., F. Bowen et N. Rondeau (1999). «Évaluation d'un programme visant le développement de la compétence sociale à la maternelle», Revue canadienne de santé mentale communautaire, 18(1), p. 77-104.

Bender, W.N. et J.K. Smith (1990). «Classroom behavior of children and adolescents with learning disabilities: A meta-analysis», Journal of Learning Disabilities, 23(5), p. 298-305.

Bennett, K. et D.R. Offord (2001). «Conduct disorder: Can it be prevented?», Current Opinion in Psychiatry, 14, p. 333-337. 
Berg, I.K. et S. Kelly (2001). Des solutions à inventer dans les services à l'enfance, Québec, EDISEM.

Berger Hepworth, E. (1991). «Parent involvement: Yesterday and today», The Elementary School Journal, 91(3), p. 209-219.

Berkowitz, L. (1993). Aggression: Its Causes, Consequences and Control, New York, McGraw-Hill.

Bishop, S.J. et Rothbaum, F. (1992). «Parents' acceptance of control needs and preschoolers' social behaviour: A longitudinal study», Canadian Journal of Behaviour Science, 24(2), p. 171-185.

Blyth, E. et J. Milner (1993). «Exclusion from school: A first step in exclusion from society?», Children and Society, 7(3), p. 255-268.

Boivin, M. (1996). «Le rôle des pairs dans le développement des problèmes intériorisés à l'enfance», dans R. Tessier, G.M. Tarabulsy et M.A. Provost (dir.), Relations sociales entre les enfants, Sainte-Foy, Presses de l'Université du Québec, p. 45-78.

Bouchard, C., M. Clarkson et R. Tessier (1996). «Méthodes», dans C. Lavallée, M. Clarkson et L. Chénard (dir.), Conduites à caractère violent dans la résolution de conflits entre proches, Montréal, Santé Québec, p. 7-20.

Bouchard, C. et R. Tessier (1996). «Conduites à caractère violent à l'endroit des enfants», dans C. Lavallée, M. Clarkson et L. Chénard (dir.), Conduites à caractère violent dans la résolution de conflits entre proches, Montréal, Santé Québec, p. 21-76.

Bouchard, J.-M. et J.-C. Kalubi (2003). «Les difficultés de communication entre intervenants et parents d'enfants vivant avec des incapacités », Éducation et francophonie, 31(1), <www. acelf.ca/revue/sommaire.phd?id=4>, consulté le 22 novembre 2005.

Bouchard, J.-M., D. Pelchat et P. Boudreault (1996a). «Les relations entre parents et intervenants: perspectives théoriques», Apprentissage et socialisation, 17(1-2), p. 21-34.

Bouchard, J.-M., D. Pelchat et P. Boudreault (1996b). «Les parents et les intervenants, où en sont leurs relations?», Apprentissage et socialisation, 17(3), p. 41-48.

Bourassa, C. et D. Turcotte (1998). «Les expériences familiales et sociales des enfants exposés à la violence conjugale: des observations tirées de leurs propos », Intervention, $\mathrm{n}^{\mathrm{o}} 107, \mathrm{p} .7-18$.

Boutin, G. et F. Le Cren (1998). «Le partenariat en éducation: un défi à relever», dans R. Pallascio, L. Julien et G. Gosselin (dir.), Le partenariat en éducation: pour mieux vivre ensemble!, Montréal, Éditions Nouvelles, p. 111-134.

Bowen, F., N. Desbiens, N. Rondeau et I. Ouimet (2000). «La prévention de la violence et de l'intimidation en milieu scolaire», dans F. Vitaro et C. Gagnon (dir.), Prévention des problèmes d'adaptation (tome II): Les problèmes externalisés, Sainte-Foy, Presses de l'Université du Québec, p. 165-214. 
Bowen, F., N. Rondeau, N. Rajotte et J. Bélanger (2000). «Évaluation d'un programme de prévention de la violence au premier cycle du primaire», Revue des sciences de l'éducation, 26(1), p. 173-196.

Breton, M. (1998). Insertion: résultats concrets de l'empowerment, Montréal, Institut de recherche pour le développement social des jeunes (texte inédit).

Breton, M. (1994). «Relating competence-promotion and empowerment», Journal of Progressive Human Services, 5(1), p. 27-44.

Bronfenbrenner, U. (1986). «Ecology of the family as a context for human development: Research perspectives», Development Psychology, 22, p. 723-742.

Bullis, M., H.M. Walker et J.R. Sprague (2001). «A promise unfulfilled: Social skills training with at-risk and antisocial children and youth», Exceptionality, 9(1-2), p. 67-90.

Burke, J., R. Loeber et B. Birmaher (2002). «Oppositional defiant disorder and conduct disorder: A review of the past 10 years », American Journal of Child and Adolescent Psychiatry, 41(1), p. 1275-1293.

Cameron, G. et S. Cadell (1997). «Empowering in prevention programs for disadvantaged children and families », Empowerment Practice in Social Work: Developing Richer Conceptual Foundations, Faculty of Social Work, Toronto, University of Toronto.

Cantin, S. (1998). «Quinze offres d'aide pour une demande», Intervention, $\mathrm{n}^{\circ} 106$, p. 35-44.

Carrington, P.J. (1999). «Trends in youth crime in Canada, 1977-1996», Canadian Journal of Criminology, 41(1), p. 1-32.

Cavell, T.A. (2000). Working with Parents of Aggressive Children: A Practitioner's Guide, Washington, American Psychological Association.

Centre de recherche et d'intervention sur la réussite scolaire (2002). «La prévention des conduites violentes en milieu scolaire: évaluer pour développer de meilleures pratiques », Bulletin, Sainte-Foy, Université Laval.

Centre de recherche et d'intervention sur la réussite scolaire (2001). «L'efficacité des programmes de prévention du décrochage scolaire », Bulletin, Sainte-Foy, Université Laval.

Centre de recherche et d'intervention sur la réussite scolaire (1993). «La réussite scolaire et la collaboration entre l'école et la famille», Bulletin, Sainte-Foy, Université Laval.

Centre national d'information sur la violence dans la famille (1997). Les jeunes et la violence, Ottawa, Santé Canada.

Charlot, B. et J.-C. Émin (2001). Violence à l'école. État des savoirs, Paris, Bordas/ VUEF.

Christenson, S.L., J.A. Hirsch et C.M. HurIey (1997). «Families with aggressive children and adolescents», dans A. Goldstein et J.-C. Conoley (dir.), School Violence Intervention: A Practical Handbook, New York, Guilford, p. 325-365. 
Christenson, S.L., T. Rounds et M.J. Franklin (1992). «Home-school collaboration: Effects, issues and opportunities», dans S.L. Christenson et J.-C. Conoley (dir.), Home-School Collaboration: Enhancing Children's Academic and Social Competence, Bethesda (Maryland), The National Association of School Psychologists, p. 19-52.

Christenson, S.L. et S.M. Sheridan (2001). Schools and Families: Creating Essential Connections for Learning, New York, Guilford.

Cifali, M. (1997). «Entretien avec Mireille Cifali», réalisé par F. Jacquet-Francillon, Recherche et formation, $\mathrm{n}^{\mathrm{o}} 25, \mathrm{p} .69-83$.

Claes, M. et J. Comeau (1996). «L'école et la famille: deux mondes?», Lien social et politiques - RIAC, $\mathrm{n}^{\circ} 35, \mathrm{p} .75-85$.

Cloutier, R., L. Champoux et C. Jacques (1997). «La pauvreté des familles et le fonctionnement du service de garde: un point de vue comparatif», dans F.V. Tochon (dir.), Éduquer avant l'école: l'intervention préscolaire en milieux défavorisés et pluriethniques, Montréal, Presses de l’Université de Montréal, p. 65-90.

Cloutier, R. et A. Renaud (1990). Psychologie de l'enfant, Montréal, Gaëtan Morin.

Cohen, M.B. (1998). «Perceptions of power in client/ worker relationships», Families in Society, 79(4), p. 433-442.

Comeau, J. et A. Salomon (1994). «Les relations école-famille: les difficultés d'une coopération», dans P. Durning et J.-P. Pourtois (dir.), Éducation et famille, Bruxelles, De Boeck, p. 206-217.

Conseil canadien de développement social (2001). Le progrès des enfants au Canada, Ottawa, Conseil canadien de développement social.

Conseil de développement de la recherche sur la famille du Québec (2002). "Qu'attend-on des parents d'aujourd'hui?», Bulletin de liaison, numéro spécial, p. 4-8.

Conseil de la famille et de l'enfance du Québec (2000). Pour une plus grande complicité entre les familles et les écoles, Québec, Conseil de la famille et de l'enfance du Québec.

Conseil national du bien-être social (2002). Profil de pauvreté 1999: seuils de faible revenu après les impôts, Ottawa, Statistique Canada, $\mathrm{n}^{\mathrm{o}} 117$.

Couture-Côté, C. (2001). Les clés pour grandir, présentation dans le cadre du cours «Enfance et difficulté», École de service social, Ottawa, Université d'Ottawa.

Cowie, R. (2000). «Aggressive and bullying behaviour in children and adolescents», dans G. Boswell (dir.), Violent Children and Adolescents: Asking the Question Why?, Londres, Whurr, p. 138-150.

Crowell, D.R. (1987). «Childhood aggression and violence: Contemporary issues», dans D.H. Crowell, I.M. Evans et C.R. O'Donnell (dir.), Childhood Aggression and Violence: Sources of Influence, Prevention and Control, New York, Plenum, p. 75-94. 
Crozier, M. et E. Friedberg (1977). L'acteur et le système: les contraintes de l'action collective, Paris, Seuil.

Cullingford, C. et M. Morrison (1999). «Relationship between parents and schools: A case study», Educational Review, 51(3), p. 253-262.

Dangel, R. et R. Polster (1984). Parent Training: Foundations of Research and Practice, New York, Guilford.

Danyluk, V. (1995). «La violence chez les jeunes: compréhension et intervention», dans Y. L'Abbé (dir.), La violence chez les jeunes: compréhension et intervention, Montréal, Sciences et Culture, p. 15-23.

Darling, N. et L. Steinberg (1993). «Parenting style as context: An integrative model», Psychological Bulletin, 113(3), p. 487-496.

Daumas-Saab, C. (2001). «Intervention en groupe auprès de parents d'enfants défiant l'autorité parentale», Intervention, 111, p. 58-66.

Day, D.M., C.A. Golench, J. MacDougall et C.A. Beals-Gonzales (1995). La prévention de la violence à l'école au Canada: résultats d'une étude nationale des politiques et programmes, Ottawa, Solliciteur général du Canada.

Debarbieux, É. (2001). «Le savant, le politique et la violence: vers une communauté scientifique européenne sur la violence à l'école», dans É. Debarbieux et C. Blaya (dir.), La violence en milieu scolaire: dix approches en Europe, Paris, ESF, p. 9-24.

Desimone, L. (1999). «Linking parent involvement with student achievement: Do race and income matter?», The Journal of Educational Research, 93(1), p. 11-30.

De Singly, F. (2000). «L'école et la famille», dans A. Van Zanten (dir.), L'école, l'état des savoirs, Paris, La Découverte, p. 271-279.

De Singly, F. (1997). «La mobilisation familiale pour le capital scolaire», dans F. Dubet (dir.), École, familles: le malentendu, Paris, Textuel, p. 45-58.

Deslandes, R. (2001). «L'environnement scolaire», dans M. Hamel, L. Blanchet et C. Martin (dir.), 6-12-17, Nous serons bien mieux!: les déterminants de la santé et du bien-être des enfants d'âge scolaire, Québec, Publications du Québec, p. 251-287.

Deslandes, R. (1999). «Une visée partenariale dans les relations entre l'école et les familles: complémentarité de trois cadres conceptuels », La revue internationale de l'éducation familiale, 3(1-2), p. 31-42.

Deslandes, R., P. Bouchard et J.-C. St-Amant (1998). «Family variables as predictors of school achievement: Sex differences in Quebec adolescents», Canadian Journal of Education, 23(4), p. 390-404.

Deslandes, R. et L. Morin (2002). Rôle des parents dans les apprentissages des enfants: attentes des parents et attentes des enseignants, texte publié sur le site Web de la $6^{\mathrm{e}}$ Communication à la $6^{\mathrm{e}}$ Biennale, Paris, 3-7 juillet, Rubrique recherche $n^{0} 165$, <www.inrp.fr/Acces / Biennale/6biennale/Contrib/affich.php ?\&m ode=long\&NUM=165>, consulté le 22 novembre 2005 . 
Deslandes, R. et M. Jacques (2004). «Relations famille-école et l'ajustement du comportement socioscolaire de l'enfant à l'éducation préscolaire», Éducation et Francophonie, 32(1), p. 172-200, <www.acelf.ca/ revue/sommaire.php?id=2>, consulté le 22 novembre 2005.

Deslandes, R. et É. Royer (1994). «Style parental, participation parentale dans le suivi scolaire et réussite scolaire», Service social, 43(2), p. 63-80.

Desmarais, D., F. Beauregard, D. Guérette, M. Hrimech, Y. Lebel, P. Martineau et S. Péloquin (2000). Détresse psychologique et insertion sociale des jeunes adultes: un portrait complexe, une responsabilité collective, Sainte-Foy, Publications du Québec.

Drolet, M. et A. Charpentier (1999). «L'empowerment psychologique et l'intervention familiale individualisée: démarche de conceptualisation et pistes pour la pratique», Revue canadienne de service social, 16(1), p. 19-34.

Drolet, M. et M. Paquin (à paraître). «Building collaboration between school and parents: Issues for school social worker and parents whose young children exhibit violent behavior at school», European Journal of Social Work, <www. tandf.co.uk/journals/online/1369-1487.asp>.

Drolet, M. et M. Paquin avec la collaboration de M. Soutyrine, R. Hasan et C. Ringuet (2003). Pour contrer la violence chez les enfants de 3 à 9 ans: une collaboration entre les écoles et parents concernés, occasions d'empowerment pour les parents, Rapport technique, texte inédit remis au Centre national de prévention du crime, Justice Canada, 205 p.

Drolet, M., M. Paquin et M. Soutyrine (2004). «Donner la parole aux parents de jeunes enfants présentant des conduites violentes à l'école: pour une pratique d'inclusion en intervention familiale», dans C. Lacharité et G. Pronovost (dir.) avec la collaboration de É. Coutu, Comprendre la famille, Québec, Presses de l'Université du Québec, p. 109-130.

Dubet, F. (1997). École, familles: le malentendu, Paris, Textuel.

Dubet, F. (1994). Sociologie de l'expérience, Paris, Seuil.

Dubet, F. et D. Martuccelli (1996). À l'école: sociologie de l'expérience scolaire, Paris, Seuil.

Duhamel-Maples, M. (1996). «La violence en milieu scolaire: un défi pour intervenants et intervenantes", Reflets, Revue ontaroise d'intervention sociale et communautaire, 2(1), p. 40-57.

Dumont, M., M.A. Provost et M. Dubé (1990). «La compétence sociale: une approche multivariée», dans M.A. Provost (dir.), Le développement social des enfants, Montréal, Agence d'ARC, p. 13-55.

Dunst, C., C. Trivette et C. Johanson (1994). «Parent-professional collaboration and partnerships», Chapter 1: «Methods, strategies and practices», Supporting and Strengthening Families, Cambridge, Brookline, p. 197-211.

Dupper, D.R. (1994). «Reducing out-of-school suspensions: A survey of attitudes and barriers », Social Work in Education, 16(2), p. 115-123. 
Dupper, D.R. et L.A. Bosch (1996). «Reasons for school suspensions», Journal for a Just and Caring Education, 2(2), p. 140-150.

Duxbury, L., C. Higgins et D. Coghill (2003). Témoignages canadiens : à la recherche de la conciliation travail-vie personnelle, Ottawa, Développement des ressources humaines Canada.

Dye, J.S. (1989). «Parental involvement in curriculum matters», Educational Research, 31(1), p. 20-35.

Eccles, J.S. et R. Harold (1993). «Parent-school involvement during the early adolescent years », Teachers College Record, 94(3), p. 568-587.

Ehrensaft, E. et M. Tousignant (2004). «L'écologie humaine et sociale de la résilience», dans CRAES-CRIPS (dir.), La résilience en action, passeport pour la santé: faire face aux difficultés et construire..., Lyon, p. 9-10, <www.craes-crips.org/ publications / publication.asp?an=2004>, consulté le 22 novembre 2005.

Eisenberg, N., I.K. Guthrie, B.C. Murphy et S.A. Shepard (1999). «Consistency and development of prosocial dispositions: A longitudinal study», Child Development, 70(6), p. 1360-1372.

Epstein, J.L. (2001). School-Family and Community Partnerships: Preparing Educators and Improving Schools, Boulder, Westview.

Epstein, J.L. (1995). «School/family/community partnerships: Caring for the children we share», Phi Delta Kappan, 76, p. 701-712.

Epstein, J.L. (1992). «School and family partnerships», dans M. Atkins (dir.), Encyclopedia of Educational Research, New York, MacMillan, p. 1139-1151.

Epstein, J.L. et S.L. Dauber (1991). «School programs and teacher practices of parent involvement in inner-city elementary and middle schools», The Elementary School Journal, 91(3), p. 289-305.

Epstein, J.L. et M.G. Sanders (1998). «What we learned from international studies of school-family community partnership», Childhood Education, 74(6), p. 392-394.

Evans, I.M., A. Okifuji, L. Engler et K. Bromley (1993). «Home-school communication in the treatment of childhood behavior problems", Child and Family Behavior Therapy, 15(2), p. 37-60.

Evans, I.M. et A.D. Scheuer (1987). «Analyzing response relationships in childhood aggression: The clinical perspective», dans D.H. Cromwell, I.M. Evans et C.R. O'Donnell (dir.), Childhood Aggression and Violence: Sources of Influence, Prevention and Control, New York, Plenum, p. 75-94.

Fabes, R.A., N. Eisenberg, S. Jones, M. Smith, I.K. Guthrie, R. Poulin, S.A. Shepard et J. Friedman (1999). «Regulation, emotionality and preschoolers, socially competent peer interactions », Child Development, 70(2), p. 432-442.

Farmer, T.W., E.M.Z. Farmer et D.M. Gut (1999). «Implications of social development research for school-based intervention for aggressive youth with EBD», Journal of Emotional and Behavioral Disorders, 7(3), p. 130-136. 
Farner, C.D. (1996). «Proactive alternatives to school suspension», Journal of Emotional and Behavioral Problems, 5(1), p. 47-51.

Farrington, D.P. (2000). «Adolescent violence: Findings and implications from the Cambridge study», dans G. Boswell (dir.), Violent Children and Adolescents: Asking the Question Why?, Londres, Whurr, p. 19-35.

Fasko, D., D.J. Grubb et J.S. Osborne (1997). «Suspensions of students with and without disabilities: A comparative study », Research in the Schools, 4(1), p. $45-50$.

Fédération nationale des conseillères et conseillers scolaires francophones (2003). Tu peux compter sur moi, Ottawa, FNCSF et CFORP.

Feucht, J.E. (1998). «Reading, writing, arithmetic and suspensions », NASSP Bulletin, 82, p. $14-15$.

Feuerstein, A. (2000). «School characteristics and parent involvement: Influences on participation in children's schools», The Journal of Educational Research, 94, p. 29-40.

Flanagan, C. (1999). Early Socialisation: Sociability and Attachment, Londres, Routledge.

Fortin, A. (1999). «La famille, premier et ultime recours», dans F. Dumont, S. Langlois et Y. Martin (dir.), Traité des problèmes sociaux, Sainte-Foy, Presses de l’Université Laval, p. 947-962.

Fortin, L. (2002). «Violence et problèmes de comportement», Enquête sociale et de santé auprès des enfants et des adolescents québécois 1999, Québec, Institut de la statistique du Québec, p. 451-470.

Fortin, L. et H. Mercier (1994). «Liens entre la participation des parents à l'école et les comportements de leur enfant en classe du primaire», Revue des sciences de l'éducation, 20(4), p. 513-527.

Fortin, L., J. Toupin, R. Pauzé, M. Déry et H. Mercier (1996). «Variables associées à la compétence scolaire des adolescents en troubles de comportement», Scientia paedagogica experimentalis, 33(2), p. 245-268.

Freedman, J.L. (2002), Media Violence and Its Effect on Aggression: Assessing the Scientific Evidence, Toronto, University of Toronto Press.

Funk, W. (2001). «La violence à l'école en Allemagne: un état des lieux», dans É. Debarbieux et C. Blaya (dir.), La violence en milieu scolaire: dix approches en Europe, Paris, ESF, p. 25-41.

Gagnon, C. et F. Vitaro (2000). «La prévention du trouble des conduites, avec centration sur les comportements violents», dans F. Vitaro et C. Gagnon (dir.), Prévention des problèmes d'adaptation chez les enfants et les adolescents (tome II). Les problèmes externalisés, Sainte-Foy, Presses de l’Université du Québec, p. 231-290.

Gareau, M. et D. Sawatzky (1995). «Parents and schools working together: A qualitative study of parent-school collaboration », The Alberta Journal of Educational Research, 61(4), p. 462-273. 
Garibaldi, A.M, L. Blanchard et S. Brooks (1996). «Conflict resolution training, teacher effectiveness and student suspension: The impact of a health and safety initiative in the New Orleans public schools ", Journal of Negro Education, 65(4), p. 408-413.

Gavin, K.M. et D.B. Greenfield (1998). «A comparison of levels of involvement for parents with at-risk African American kindergarten children in classrooms with high versus low teacher encouragement », Journal of Black Psychology, 24(4), p. 403-417.

Gaziel, H.H. et M.M. Warnet (1996). «Attitudes et intérêts des parents à l'égard de l'école: impacts sur les résultats scolaires», Sciences de l'éducation pour l'ère nouvelle, 29(3), p. 77-93.

Georgiou, S.N. (1999). «Parental attributions as predictors of involvement and influences on child achievement», British Journal of Educational Psychology, 69 , p. 409-429.

Gettinger, M. et K. Waters-Guetschow (1998). «Parental involvement in schools: Parent and teacher perceptions of roles, efficacy and opportunities », Journal of Research and Development in Education, 32(1), p. 38-52.

Glasman, D. (1994). «Les familles défavorisées face à l'école», dans P. Durning et J.-P. Pourtois (dir.), Éducation et famille, Bruxelles, De Boeck, p. 218-234.

Gohier, C. (1996). «Identité professionnelle et formation des maîtres: le pourquoi, le quoi et le comment», L'éducation en recherche, les nouveaux défis, Montréal, Université du Québec à Montréal, p. 241-249.

Gorman, T. (1998). «Social class and parental attitudes toward education. Resistance and conformity to schooling in the family», Journal of Contemporary Ethnography, 27(1), p. 10-44.

Goupil, G. (1997a). Communications et relations entre l'école et la famille, Montréal, Chenelière/McGraw-Hill.

Goupil, G. (1997b). Les élèves en difficulté d'adaptation et d'apprentissage, Montréal, Gaëtan Morin.

Griffith, J. (1998). "The relation of school structure and social environment to parent involvement in elementary schools», The Elementary School Journal, 99(1), p. 53-80.

Grolnick, W.S., C. Benjet, C.O. Kurowski et N.H. Apostoleris (1997). «Predictors of parent involvement in children's schooling ", Journal of Educational Psychology, 89(3), p. 538-548.

Grolnick, W.S., S.T. Gurland, G.K. Jacob et W. Decourcey (2002). «The development of self-determination in middle childhood and adolescence», dans A. Wigfield et J.S. Eccles (dir.), Development of Achievement Motivation, San Diego, Academic Press, p. 148-174.

Grolnick, W.S. et M.L. Slowiaczek (1994). «Parents' involvement in children's schooling: A multidimensional conceptualization and motivational model», Child Development, 65(1), p. 237-252. 
Guindon, J. (1992). Developing an In-school Suspension Program in an Elementary School as an Alternative to Home-bound Suspension, Practicum Report, Fort Lauderdale, Nova University.

Gutiérrez, L.M., K.A. DeLois et L. GlenMaye (1995). «Understanding empowerment practice: Building on practitioner based knowledge», Families in Society, 76(9), p. 534-544.

Gutiérrez, L.M. (1994). «Understanding the empowerment process: Does consciousness make a difference? », Social Work Research, 19(4), p. 229-237.

Hamel, P. (2001). «Action collective et nouvelle culture politique», dans H. Dorvil et R. Mayer (dir.), Problèmes sociaux (tome II): Études de cas et interventions sociales, Sainte-Foy, Presses de l'Université du Québec, p. 429-446.

Hartman, R. et S.A. Stage (2000). «The relationship between social information processing and in-school suspension for students with behavioral disorders », Behavioral Disorders, 25(3), p. 183-195.

Hasan, R., M. Drolet et M. Paquin (2003). «Les conduites violentes chez les 3 à 6 ans: comprendre pour mieux intervenir », Reflet, Revue ontaroise d'intervention sociale et communautaire, 9(1), p. 150-177.

Hayden, C. (1994). «Primary age children excluded from school: A multi agency focus for concern», Children and Society, 8(3), p. 257-273.

Hayden, C. et D. Ward (1996). «Faces behind the figures: Interviews with children excluded from primary school», Children and Society, 10(4), p. 255-266.

Hébert, J. (2001). «La violence à l'école: une analyse complémentaire», dans H. Dorvil et R. Mayer (dir.), Problèmes sociaux (tome II): Études de cas et interventions sociales, Sainte-Foy, Presses de l'Université du Québec, p. 155-185.

Herrenkohl, T.I., B. Huang, R. Kosterman, J.D. Hawkins, R.F. Catalano et B.H. Smith (2001). «A comparison of social development processes leading to violent behavior in late adolescence for childhood initiators and adolescent initiators of violence", Journal of Research in Crime and Delinquency, 38(1), p. 45-63.

Higgins, C. et L. Duxbury (2002). Enquête nationale sur le conflit entre le travail et la vie personnelle, Ottawa, Santé Canada.

Hirschhorn, M. (2001). «Consumérisme scolaire et démocratie», dans R. Boudon, N. Bulle et M. Cherkaoui (dir.), École et société: les paradoxes de la démocratie, Paris, Presses universitaires de France, p. 81-98.

Hoff, L.A. (1994). Les questions relatives à la violence: un guide de formation interdisciplinaire à l'intention des professionnels de la santé, Ottawa, Santé Canada.

Hoover-Dempsey, K.V., O. Bassler et J. Brissie (1992). «Explorations in parent-school relations », Journal of Educational Research, 85(5), p. 287-294.

Huberman, M.A. et M.B. Miles (1991). Analyse des données qualitatives: recueil de nouvelles méthodes, Bruxelles, De Boeck. 
Huesmann, L.R., J. Moise-Titus, C.-L. Podolski et L.D. Eron (2003). «Longitudinal relations between children's exposure to TV violence and their aggressive and violent behavior in young adulthood: 1977-1992 », Developmental Psychology, 39(2), p. 201-221.

Imich, A.J. (1994). «Exclusions from school: Current trends and issues », Educational Research, 36(1), p. 3-11.

Institut Vanier de la famille (2000). Profil des familles II, Ottawa, IVF.

Jeffrey, D. et C. Simard (2000). Enseigner et punir, Sainte-Foy, Presses de l'Université Laval, p. 38-42.

Jodelet, D. (1997). «Représentations sociales: un domaine en expansion», Les représentations sociales, Paris, Presses universitaires de France, p. 47-78.

Johnson, G.M. (1994). «Family characteristics and parental school involvement», Family Therapy, 21(1), p. 25-33.

Juneau, J. et L.-P. Boucher (2004). «Le déficit de l'attention et de l'hyperactivité (TDA/H) et les comportements violents des jeunes en milieu scolaire», Éducation et francophonie, 32(1), p. 38-53, <www.acelf.ca/revue/sommaire. phd ?id=2>, consulté le 22 novembre 2005.

Jutras, S. (1999). «Difficultés vécues dans de nouvelles structures familiales: état des recherches récentes », dans J. Alary, S. Jutras, Y. Gauthier et J. Goudreau (dir.), Familles en transformation: récits de pratiques en santé mentale, Boucherville, Gaëtan Morin, p. 1-15.

Kauffman, J.M. (1997). Characteristics of Emotional and Behavioral Disorders of Children and Youth, 6e éd., Upper Saddle River (N.J.), Merrill, Prentice-Hall.

Kay, P., M. Fitzgerald et S. McConaughy (2002). «Building effective parent-teacher partnerships», dans B. Algozzine et P. Kay (dir.), Preventing Problem Behaviors: A Handbook of Successful Prevention Strategies, Thousands Oaks, Corwin, p. 104-125.

Keith, T.Z., P.B. Keith, K.J. Quirk, J. Sperduto, S. Santillo et S. Killings (1998). «Longitudinal effects of parent involvement on high school grades: Similarities and differences across gender and ethnic groups », Journal of School Psychology, 36(3), p. 335-363.

Kieffer, C.H. (1984). "Citizen empowerment: A developmental perspective», Prevention in Human Services, 3(2-3), p. 9-36.

Kinnear, K.L. (1995). Violent Children: A Reference Handbook, Santa Barbara, ABCCLIO.

Kirkey, S. (2002). "Sleepless school children face serious health risks», Ottawa Citizen, 11 nov., p. 1-2.

Kohl, G.O., L.J. Lengua et R.J. McMahon (2000). «Parent involvement in school conceptualizing multiple dimensions and their relations with family and demography risk factors ", Journal of School Psychology, 38(6), p. 501-523. 
Kosterman, R., J.W. Graham, J.D. Hawkins, R.F. Catalano et T.I. Herrenkohl (2001). "Childhood risk factors for persistence of violence in the transition to adulthood: A social development perspective», Violence and Victims, 16(4), p. 355-369.

Kratzer, L. et S. Hodgins (1997). «Adult outcomes of child conduct problems: A cohort study», Journal of Abnormal Child Psychology, 25, p. 65-81.

L'Abbé, Y. (1995). La violence chez les jeunes: compréhension et intervention, Montréal, Sciences et Culture.

Ladd, G.W. (1999). «Peer relationships and social competence during early and middle childhood», Annual Review of Psychology, 50, p. 333-359.

Lafrenière, P.J. et J.E. Dumas (1996). «L'anxiété et le retrait social en période préscolaire: Un lien avec les relations parent-enfant», dans G.M. Tarabulsy et R. Tessier (dir.), Le développement émotionnel et social de l'enfant, Sainte-Foy, Presses de l'Université du Québec, p. 33-67.

Lahire, B. (1995). Tableaux de familles: heurs et malheurs scolaires en milieux populaires, France, Seuil/Gallimard.

Landy, S. et K.K. Tam (1996). «Yes, parenting does make a difference to the development of children in Canada», Growing Up in Canada: National Longitudinal Survey of Children and Youth, Ottawa, Développement des ressources humaines Canada, p. 103-118.

Lasnier, F. (2000). Réussir la formation par compétences, Montréal, Guérin.

Lareau, A. (1987). «Social class differences in family-school relationships: The importance of cultural capital», Sociology of Education, 60, p. 73-85.

Lareau, A. et E. McNamara-Horvat (1999). «Moments of social inclusion and exclusion: Race class and cultural capital in family-school relationships», Sociology of Education, 72, p. 37-53.

Larose, F., J. Bédard, J. et B. Terrisse (2002). «Les représentations des compétences attendues ou détenues par les élèves lors de leur transition au secondaire: enjeux dans un contexte de réforme curriculaire», Revue suisse des sciences de l'éducation, 24(2), p. 19-31.

Lauwrence, B. et C. Hayden (1995). Primary School Children Excluded from School: Numbers, Characteristics, Reasons and Circumstances, paper presented at the European Conference on Educational Research, Bath (R.-U.).

Lawler, E. et Y. Jeongkoo (1995). «Structural power and emotional processes in negociation ", Negotiation as a Social Process, Thousand Oaks, Sage.

Lazard, A. et F. Slostad (1999). «How to overcome obstacles to parent-teacher partnerships », Clearing House, 72(4), p. 206-210.

Leblanc, M. (1999). «La délinquance des adolescents», dans F. Dumont, S. Langlois et Y. Martin (dir.), Traité des problèmes sociaux, Sainte-Foy, Presses de l'Université Laval, p. 279-299. 
Le Bossé, Y. (1996). «Empowerment et pratiques sociales: illustration du potentiel d'une utopie prise au sérieux», Nouvelles pratiques sociales, 9(1), p. 127-147.

Le Bossé, Y. (1995). Étude exploratoire du phénomène de l'empowerment, thèse de doctorat, Sainte-Foy, Université Laval.

L'Écuyer, R. (1985). «L'analyse de contenu: notion et étapes », dans J.-P. Deslauriers (dir.), La recherche qualitative: résurgence et convergences, Chicoutimi, Université du Québec à Chicoutimi, p. 65-88.

Lee, J. (1994). The Empowerment Approach to Social Work Practice, New York, Columbia University Press.

Lee Yao, E. (1993). «Strategies for working effectively with Asian immigrant parents », Families in Schools in a Pluralistic Society, Albany, State University of New York Press, p. 149-156.

Lemay, M. (1995). «Les enfants violents à l'école primaire: qui sont-ils et que deviennent-ils? », dans Y. L'Abbé (dir.), La violence chez les jeunes: compréhension et intervention, Montréal, Sciences et culture, p. 27-35.

Lemonick, M.D. (2002). «Germany's Columbine», Time, Canadian Edition, 6 mai, p. 26-27.

Loeber, R., D.P. Farrington, M. Stouthhammer-Loeber et W.B. Van Kammen (1998). Antisocial Behavior and Mental Health Problems: Explanatory Factors in Childhood and Adolescence, Mahwah, Lawrence Erlbaum.

Loeber, R. et D.P. Farrington (2000). «Young children who commit crime: Epidemiology, development, risk factors, early interventions and policy implication », Development and Psychopathology, 12, p. 737-762.

Lorrain, J.-L. (1999). Les violences scolaires, Paris, Presses universitaires de France.

Lytton, H. et M. Pyryt (1998). «Predictors of achievement in basic skills: A Canadian effective schools study», Canadian Journal of Education, 23(3), p. 281-301.

Marcon, R.A. (1999). «Positive relationships between parent school involvement and public school inner-city preschoolers' development and academic performance», School Psychology Review, 28(3), p. 395-412.

Martichoux, J. (2000). Violence des jeunes: les parents sont-ils démissionnaires?, Issyles-Moulineaux, Prat.

Martin, J., L. Tett et H. Kay (1999). «Developing collaborative partnerships: Limits and possibilities for schools, parents and community education», International Studies in Sociology of Education, 9(1), p. 59-75.

Masten, A.S. et J.D. Coatsworth (1998). «The development of competence in favorable and unfavorable environments: Lessons from research on successful children », American Psychologist, 53(2), p. 205-220.

Mayer, R., F. Ouellet, M.-C. Saint-Jacques et D. Turcotte (2000). Méthodes de recherche en intervention sociale, Montréal, Gaëtan Morin.

McAndrew, M. (2001). Immigration et diversités à l'école, Montréal, Presses de l'Université de Montréal. 
McCain, M.N. et J.-F. Mustard (1999). Inverser la véritable fuite des cerveaux: étude sur la petite enfance, Toronto, Publications de l'Ontario.

McCarthey, S.J. (2000). «Home-school connections: A review of the literature», The Journal of Educational Research, 93(3), p. 145-153.

Mellard, D. et L. Seybert (1996). Voices about School Suspension, Expulsion and Learning, Final Report, Topeka, Kansas State Board of Education.

Ministère de la Justice du Canada (2002). La Loi sur le système de justice pénale pour les adolescents: une nouvelle loi, une nouvelle approche, document d'information, Ottawa, Gouvernement du Canada.

Ministère de la Justice du Canada (1998). La stratégie fédérale en matière de justice pour les jeunes, document d'information, Ottawa, Gouvernement du Canada.

Ministère de l'Éducation de l'Ontario (2001a). Écoles de l'Ontario: code de conduite, Toronto, Gouvernement de l'Ontario.

Ministère de l'Éducation de l'Ontario (2001b). Éducation de l'enfance en difficulté: guide pour les éducatrices et éducateurs, Partie A: Lois et politiques, Toronto, Gouvernement de l'Ontario.

Ministère de l'Éducation de l'Ontario (2001c). En bref: participation des parents à la vie des écoles, Toronto, Gouvernement de l'Ontario.

Ministère de l'Éducation de l'Ontario (2001d). Rapport aux conseils d'école sur l'éducation, Toronto, Gouvernement de l'Ontario.

Ministère de l'Éducation de l'Ontario (2001e). Les programmes de discipline rigide, document d'information, Toronto, Gouvernement de l'Ontario.

Ministère de l'Éducation de l'Ontario (2000). L'Ontario fixe des normes pour accrôttre la sécurité dans les écoles, document d'information, Toronto, Gouvernement de l'Ontario.

Ministère de l'Éducation et de la Formation de l'Ontario (1994). Pour des écoles sans violence: une politique, Toronto, Gouvernement de l'Ontario.

Ministère de l'Éducation du Québec (1992). Interprétation des définitions des élèves handicapés ou en difficulté d'adaptation ou d'apprentissage, Québec, Gouvernement du Québec.

Mishna, F. (2003). «Learning disabilities and bullying: Double jeopardy », Journal of Learning Disabilities, 36(4), p. 336-347.

Montandon, C. (1996). «Les relations des parents avec l'école», Lien social et politiques - RIAC, nº 35, p. 63-73.

Montandon, C. (1994). «Les relations parents-enseignants dans l'école primaire: de quelques causes d'incompréhension mutuelle», dans P. Duming et J.-P. Pourtois (dir.), Éducation et famille, Bruxelles, De Boeck, p. 189-205.

Montandon, C. et P. Perrenoud (1994). Entre parents et enseignants: un dialogue impossible?», Paris, Lang.

Moreau, M. et L. Léonard (1993). Empowerment II : Snapshots of the Structural Approach in Action, Ottawa, Bibliothèque nationale du Canada. 
Nagin, D.S. et R.E. Tremblay (2001). «Parental and early childhood predictors of persistent physical aggression in boys from kindergarten to high school», Archives of General Psychiatry, 58, p. 389-394.

Nagin, D.S. et R.E. Tremblay (1999). «Trajectories of boys' physical aggression, opposition and hyperactivity on the path to physically violent and nonviolent juvenile delinquency », Child Development, 70(5), p. 1181-1196.

Nakagawa, K. (2000). «Unthreading the ties that bind: Questioning the discourse of parent involvement», Education Policy, 14(4), p. 443-472.

Normandeau, S. et I. Nadon (2000). «La participation des parents à la vie scolaire d'enfants de deuxième année», Revue des sciences de l'éducation, 26(1), p. 151-172.

Nugent, J.K. (1992). «L'impact du père sur le développement du bébé dans la première année critique de la vie», dans E.J. Anthony et C. Chiland (dir.), L'enfant dans sa famille: le développement en péril, Paris, Presses universitaires de France, p. 153-183.

Offord, D.R. et E.L. Lipman (1996). «Emotional and behavioural problems», Growing Up in Canada: national Longitudinal Survey of Children and Youth, Ottawa, Développement des ressources humaines Canada, p. 119-126.

Ohsako, T. (1997). «Tackling school violence worldwide: A comparative perspective of basic issues and challenges », Violence at School, Global Issues and Interventions, Paris, United Nations Educational, Scientific and Cultural Organization, p. 7-19.

Organisation de coopération et de développement économiques (1997). Les parents partenaires de l'école, Paris, Centre pour la recherche sur l'innovation dans l'enseignement.

Oser, E. et A. Bandura (1990). «Mechanisms governing empowerment effects: A self-efficacy analysis», Journal of Personality and Social Psychology, 58(3), p. 472-496.

Palermo, G.B. (1994). The Faces of Violence, Springfield, Charles C. Thomas.

Paquin, M. (2004). «Liminaire: la violence en milieu scolaire: une problématique qui concerne la famille, l'école et la communauté, voire la société», Éducation et francophonie, numéro spécial sur la violence à l'école, 32(1), p. 1-14, <www. acelf.ca/revue/32-1-2004/index.html>.

Paquin, M. et M. Drolet (2000). «Les conceptions contemporaines de l'enfance à risque: commentaires critiques", L'enfance et la jeunesse à risque, Ottawa, Conseil des ministres de l'éducation du Canada, p. 8, 35.

Paquin, M. et M. Drolet (2004). «Comportements violents chez l'enfant en Ontario: problématique de la suspension scolaire externe, perceptions des parents et alternative possible », Éducation et francophonie, numéro spécial sur la violence à l'école, 32(1), p. 201-223, <www.acelf.ca/ revue/32-1-2004/index.html>. 
Paquin, M. et M. Drolet (2005). «Travail de collaboration entre les parents et les autres intervenants », dans L. Massé, N. Desbiens et C. Lanaris (dir.), Les troubles de comportement à l'école: prévention, évaluation et intervention, Boucherville, Gaëtan Boucher éditeur, p. 297-307.

Paquin, M., S. Papillon et R. Rousseau (1996). «Effets d'un programme d'assistance éducative auprès de parents ayant un enfant en difficulté scolaire», Apprentissage et socialisation, 17(1-2), p. 91-104.

Parsloe, P. (1995). «The concept of empowerment in social work practice», Hong Kong Journal of Social Work, 29(1), p. 1-11.

Patterson, G.R. (1992). Antisocial Boys, Eugene, Castalia.

Paulson, S.E. (1994). «Relations of parenting style and parental involvement with ninth-grade students' achievement», Journal of Early Adolescence, 14(2), p. 250-267.

Pearl, D. (1987). «Familial, peer and television influences on violent behaviour», dans D.H. Cromwell, I.M. Evans et C.R. O'Donnell (dir.), Childhood Aggression and Violence: Sources of Influence, Prevention and Control, New York, Plenum, p. 231-247.

Pelco, L.E., L. Jacobson, R.R. Ries et S. Melka (2000). «Perspectives and practices in family-school partnerships: A national survey of schools psychologists », School Psychology Review, 29(2), p. 235-250.

Peña, D. (2000). «Parent involvement: Influencing factors and implications », The Journal of Educational Research, 94(1), p. 42-54.

Perrenoud, P. (2001a). «Exigences excessives des parents et attitudes défensives des enseignants: un cercle vicieux», Résonances, $n^{\circ} 7$, p. 3-6, <www.unige.ch/ fapse/SSE / teachers/perrenoud/php_main/php_2001/2001_05 .html>.

Perrenoud, P. (2001b). Quelques conditions d'un partenariat durable entre les parents et les enseignants, <www.unige.ch/fapse/SSE/teachers/perrenoud/php_main /php_2002/2002_04.html>, consulté le 22 novembre 2005.

Phillips-Smith, E., C.M. Connell, G. Wright, M. Sizer, J.M. Norman, A. Hurley et S.N. Walker (1997). «An ecological model of home, school and community partnerships: Implications for research and practice», Journal of Educational and Psychological Consultation, 8(4), p. 339-360.

Pinderhughes, E. (1995). «Empowering diverse population: Family practice in the 21st century », Families in Society, 76(3), p. 131-140.

Potvin, P., R. Deslandes, P. Beaulieu, D. Marcotte, L. Fortin, É. Royer et D. Leclerc (1999). «Risque d'abandon scolaire, style parental et participation parentale au suivi scolaire», Revue canadienne de l'éducation, 24(3), p. 441-453.

Pourtois, J.-P. et H. Desmet (1997). «Les relations famille-école: un point de vue partenarial», dans F.V. Tochon (dir.), Éduquer avant l'école, Bruxelles, De Boeck.

Provost, M.A. (1990). Le développement social des enfants, Montréal, Agence d'ARC. 
Prêteur, Y., O. Lescarret et M. de Léonardis (1998). «Family education, child-parent interactions and child development », European Journal of Psychology of Education, 13(3), p. 461-474.

Quéniart, A. (2001). «Le désengagement paternel: un phénomène social aux multiples visages», dans H. Dorvil et R. Mayer (dir.), Problèmes sociaux (tome II): Études de cas et interventions sociales, Sainte-Foy, Presses de l'Université du Québec, p. 81-102.

Quintero, E. (1999). «The new faces of head start: Learning from culturally diverse families », Early Education and Development, 10(3), p. 475-497.

Raffaele, L.M. et H.M. Knoff (1999). «Improving home-school collaboration with disadvantaged families: Organizational principles, perspectives and approaches », School Psychology Review, 28(3), p. 448-466.

Rappaport, J. (1987). «Terms of empowerment/exemplars of prevention: Toward a theory for community psychology", The Journal of Contemporary Human Services, 76(3), p. 131-140.

Reid, J.B., J.M. Eddy, R.A. Fetrow et M. Stoolmiller (1999). «Description and immediate impacts of a preventive intervention for conduct problems », American Journal of Community Psychology, 27(4), p. 483-517.

Reynolds, A.J. (1999). «Educational success in high-risk settings: Contributions of the Chicago longitudinal study», Journal of School Psychology, 37(4), p. 345-354.

Reynolds, A.J. (1992). «Comparing measures of parental involvement and their effects on academic achievement», Early Childhood Research Quarterly, 7(3), p. 441-462.

Reynolds, A.J. et H. Walberg (1992). «A structural model of science achievement and attitude: An extension to high school», Journal of Educational Psychology, 84 , p. 371-382.

Reynolds, A.J., R. Weissberg et W. Kasprow (1992). «Prediction of early social and academic adjustement of children from the inner city», American Journal of Community Psychology, 20(5), p. 599-624.

Rich, D. (1993). «Building the bridge to reach minority parents: Education infrastructure supporting success for all children ", Families in Schools in a Pluralistic Society, Albany, State University of New York Press, p. 235-244.

Rich, J.M. (1992). «Predicting and controlling school violence», Contemporary Education, 64, p. 35-39.

Rooney, R.H. (1992). Strategies for Work with Involuntary Clients, New York, Columbia University Press.

Royer, É. (1995). «Behavior disorders, suspension and social skills: Punishment is not education », Therapeutic Care and Education, 4, p. 32-36.

Royer, É., I. Bitaudeau et L. Saint-Laurent (1993). «Être suspendu de l'école à 14 ans: pratiques et prévention de l'exclusion scolaire», Revue canadienne de psychoéducation, 22, p. 57-69. 
Royer, É., S. Moisan, L. Saint-Laurent, J. Giasson et A. Boisclair (1992). «Abandon scolaire: causes et remèdes ", Vie pédagogique, 80, p. 14-18.

Royer, É., C. Morand, N. Desbiens, S. Moisan et I. Bitaudeau (2000). «Fonctionnement familial et compétence sociale des élèves en difficulté de comportement à l'école secondaire», Revue des sciences de l'éducation, 36(1), p. 219-236.

Royer, É., L. Saint-Laurent, I. Bitaudeau et S. Moisan (1995). «Réussite scolaire et collaboration entre l'école et la famille», Éduquer et former: théories et pratiques, 1, p. 23-34.

Rubin, K.H. (1996). «La prédiction du comportement parental: les influences du contexte, des facteurs psychosociaux et des croyances des parents», dans G.M. Tarabulsy et R. Tessier (dir.), Le développement émotionnel et social de l'enfant, Sainte-Foy, Presses de l'Université du Québec, p. 11-32.

Saint-Jacques, M.-C., S. Drapeau et R. Cloutier (2001). «La prévention des problèmes d'adaptation chez les jeunes de familles séparées ou recomposées», dans F. Vitaro et C. Gagnon (dir.), Prévention des problèmes d'adaptation chez les enfants et les adolescents (tome 1): Les problèmes internalisés, Sainte-Foy, Presses de l’Université du Québec, p. 353-388.

Saint-Laurent, L., J. Giasson, C. Simard, J.J. Dionne et É. Royer (1995), Programme d'intervention auprès d'élèves à risque: une nouvelle option éducative, Montréal, Gaëtan Morin.

Saint-Laurent, L., É. Royer, M. Hébert et L. Tardif (1994). «Enquête sur la collaboration famille-école», Revue canadienne de l'éducation, 19(3), p. 270-286.

Salomon, A. et J. Comeau (1998). «La participation des parents à l'école primaire trente ans après: un objectif encore à atteindre», International Review of Education, 44(2-3), p. 251-267.

Saravanamuttoo, H. (2001). «How Ontario will spend its federal allocation under the children's agenda", Bulletin de l'Association des travailleurs sociaux de l'Ontario. Section de l'Est, 27(3), p. 11-14.

Schick, A. (2002). «Behavioural and emotional differences between children of divorce and children from intact families: Clinical significance and mediating processes », Swiss Journal of Psychology, 61(1), p. 5-14.

Schiele, B. et L. Boucher (1997). «L'exposition scientifique: une manière de représenter la science», dans D. Jodelet (dir.), Les représentations sociales, Paris, Presses universitaires de France, p. 429-447.

Schreiber, E.H. et K.N. Schreiber (2002). "A study of parents of violent children», Psychological Report, 90(1), p. 101-104.

Sécurité publique et Protection civile Canada (2005). L'incidence de l'intimidation au Canada, <www.eric.ca/sasslf/web/iquebec/fdc/themes/pafa/ tmp979591484.htm>, consulté le 22 novembre 2005.

Sheridan, S., T. Kratochwill et S. Elliott (1990). «Behavioral consultation with parents and teachers: Delivering treatment for socially withdrawn children at home and school», School Psychology Review, 19(1), p. 33-52. 
Stage, S.A. (1997). «A preliminary investigation of the relationship between inschool suspension and the disruptive classroom behavior of students with behavioral disorders», Behavioral Disorders, 23(1), p. 57-76.

Steinberg, L., J.D. Elmen et N.S. Mounts (1989). «Authoritative parenting, psychosocial maturity and academic success among adolescents », Child Development, 60, p. 1424-1436.

Stormont, M. (2000). «Early child risk factors for externalizing and internalizing behaviours: A 5-year follow forward assessment», Journal of Early Intervention, 23(3), p. 180-190.

Stratégie nationale pour la prévention du crime (2002). <www.psepc-sppcc.qc.ca/ prq/cp/ncps-fr.asp >, consulté le 22 novembre 2005.

Straus, A. et J. Corbin (1990). Basics of Qualitative Research, Grounded Theory Procedures and Techniques, Newbury Park, Sage.

Straus, M.A. et R.J. Gelles (1990). Physical Violence in American Families: Risk Factors and Adaptations to Violence in 8,145 Families, Piscataway (N.J.), Transaction Publishers.

Sullivan, J.S. (1989). «Elements of a successful in-school suspension program», NASSP Bulletin, 73, p. 32-38.

Tarabulsy, G.M. et R. Tessier (1996). Le développement émotionnel et social de l'enfant, Sainte-Foy, Presses de l’Université du Québec.

Tessier, R., G.M. Tarabulsy et M.A. Provost (1996). Relations sociales entre les enfants, Sainte-Foy, Presses de l’Université du Québec.

Tremblay, D.-G. (2003). «Articulation emploi-famille et temps de travail», Bulletin du Conseil de développement de la recherche sur la famille du Québec, 4(1), p. 2-5.

Tremblay, R.E. (2000). «The origins of youth violence», Isuma, p. 19-24.

Tremblay, R.E. (1995). «Les enfants violents à l'école primaire: qui sont-ils et que deviennent-ils? », dans Y. L'Abbé (dir.), La violence chez les jeunes : compréhension et intervention, Montréal, Sciences et culture, p. 129-148.

Tremblay, R.E., B. Boulerice, P.W. Harden, P. McDuff, D. Pérusse, R.O. Pihl et M. Zoccolillo (1996). «Do children in Canada become more aggressive as they approach adolescence?», Growing Up in Canada: national Longitudinal Survey of Children and Youth, Ottawa, Développement des ressources humaines Canada, p. 127-137.

Trépanier, J. (1999). «La justice des mineurs au Canada: remises en question à la fin d'un siècle», Criminologie, 32(2), p. 7-35.

Trethewey, A. (1997). «Resistance, identity and empowerment: A postmodem feminist analysis for clients in human service organization », Communication Monographs, 64, p. 281-301.

Trudelle, D. et E. Montambault (1994). «Le sentiment de compétence éducative parentale chez des parents d'enfants d'âge préscolaire présentant des problèmes de comportement», Service social, 43(2), p. 47-80. 
Turcotte, D., G. Lamonde et J. Lindsay (2002). La prévention de la violence à l'école primaire par la promotion des comportements pacifiques: évaluation du programme Les ambassadeurs et ambassadrices de la paix, Centre de recherche interdisciplinaire sur la violence familiale et la violence faite aux femmes, Collection études et analyses, Sainte-Foy, Université Laval.

Turcotte, D., A. St-Amand, A. Beaudoin et L. Champoux (2001). «Évaluation d'une démarche d'intervention pour les enfants d'âge préscolaire présentant des problèmes de comportement», Intervention, $\mathrm{n}^{\mathrm{o}} 113$, p. 27-37.

Uchitelle, S., D. Bartz et L. Hillman (1989). «Strategies for reducing suspensions », Urban Education, 24(2), p. 163-176.

Vanderslice, R. (1999). Developing Effective In-school Suspension Programs, paper presented at the Annual Meeting of Women in Educational Leadership, Bicton, Western Australia.

Van Neste, M. (1994). «La réussite éducative et la suspension de l'élève: un antagonisme irréconciliable? », Vie pédagogique, 80, p. 25-28.

Van Zanten, A. (2001). L'école de la périphérie: scolarité et ségrégation en banlieue, Paris, Presses universitaires de France.

Van Zanten, A. et M. Duru-Bellat (1999). Sociologie de l'école, Paris, Armand Colin.

Vatz Laaroussi, M. (1996). «Les nouveaux partenariats famille-école au Québec: l'extériorité comme stratégie de survie des familles défavorisées? », Lien social et politiques - RlAC, $\mathrm{n}^{\circ} 35$, p. 87-97.

Verlinden, S., M. Hersen et J. Thomas (2000). «Risk factors in school shootings », Clinical Psychology Review, 20(1), p. 3-56.

Vézina, A., R. Bradet, M. Lord, D. Pelletier et M. Thibault (1995). Diagnostic et traitement de l'enfant en danger, Paris, L'Harmattan.

Viau, R. (1999). «Catégorisation des facteurs environnementaux qui influencent la motivation des élèves en contexte scolaire», Apprentissage et socialisation, 19(2), p. 65-79.

Viau, Y. (2001). Le Canada renouvelle son système de justice pour les jeunes, Actes du Symposium international sur la jeunesse, Hull, Ville de Hull, p. 40-43.

Vincent, C. (1996). «Parent empowerment? Collective action and inaction in education », Oxford Review of Education, 22(4), p. 465-482.

Vincent, C. et S. Tomlinson (1997). «Home-school relationships: The swarming of the disciplinary mechanisms?», British Educational Research Journal, 23(3), p. 361-377.

Vitaro, F. et C. Gagnon (2000). Prévention des problèmes d'adaptation chez les enfants et les adolescents (tome II): Les problèmes externalisés, Sainte-Foy, Presses de l’Université du Québec. 
Webster-Stratton, C. (1998). «Preventing conduct problems in head start children: Strengthening parenting competencies», Journal of Consulting and Clinical Psychology, 66(5), p. 715-730.

Webster-Stratton, C. (1993). «Strategies for helping early school-aged children with oppositional defiant and conduct disorders: The importance of home-school partnerships», School Psychology Review, 22(3), p. 437-457.

Webster-Stratton, C., J.B. Reid et M. Hammond (2001). «Preventing conduct problems, promoting social competence: A parent and teacher training partnership in head start», Journal of Clinical Child Psychology, 30(3), p. 283302.

Zelman, G. et J. Waterman (1998). «Understanding the impact of parent-school involvement on children's educational outcomes", Journal of Educational Research, 91(6), p. 370-380.

Zimmerman, M.A. (1995). «Psychological empowerment: Issues and illustrations», American Journal of Community Psychology, 23(5), p. 581-599.

Zimmerman, M.A. et J. Rappaport (1988). «Citizen participation, perceived control and psychological empowerment », American Journal of Community Psychology, 16(5), p. 725-750. 



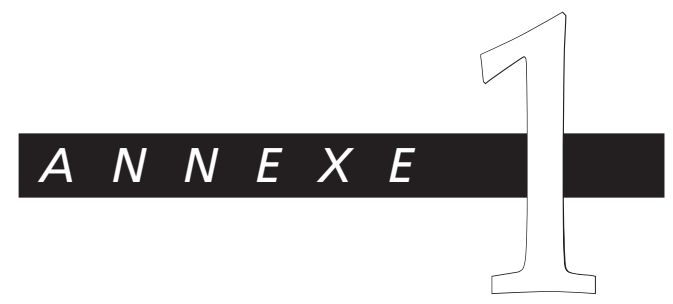

La grille d'entrevue parentale 



\section{PARTIE 1}

\section{CERNER LES PERCEPTIONS DES PARENTS AU SUJET} DES COMPORTEMENTS VIOLENTS DE LEUR ENFANT ENVERS LEURS PAIRS AU PRÉSCOLAIRE ET AU PRIMAIRE

1. Votre enfant présente-t-il l'une ou l'autre des caractéristiques suivantes?
$\square$ n'écoute pas (attention)
$\square$ n'est pas capable de se concentrer pour faire une
$\square$ change d'humeur facilement (variable) tâche
$\square$ grogne (chiale)
$\square$ agit en bébé (maturité)
$\square$ s'oppose (conteste)
$\square$ réplique (avoir le dernier mot)
$\square$ est anxieux (insécure)
$\square$ est impulsif (prompt)
$\square$ est déprimé (triste)
$\square$ autre
$\square$ bouge beaucoup (hyperactif)

2. De quelle manière s'est conduit votre enfant à l'école (avec ses pairs)?

$\square$ insulte

$\square$ malmène et intimide

$\square$ blâme

$\square$ menace de frapper ou d'envoyer quelque chose par la tête

$\square$ lance, casse ou donne des coups de pied sur un objet $\square$ frappe, mord

$\square$ donne des coups de pied

$\square$ donne des coups de poing

$\square$ se bat avec les autres

$\square$ détruit ses propres choses ou celles des autres

$\square$ autre

3. À quelle fréquence se produisent ces comportements?

$\square 1$ fois par jour

$\square 1$ fois par semaine

$\square$ plusieurs fois par semaine

$\square 1$ fois par mois $\square$ plusieurs fois par mois

$\square 1$ fois par saison

$\square$ autre 
4. Lequel (lesquels) de ces comportements a (ont) mené à une suspension ou une possibilité de suspension scolaire?

5. Depuis quand votre enfant se comporte-t-il de cette manière à l'école?

6. Est-ce que votre enfant se comporte de la même manière à la maison ou dans l'entourage qu'à l'école?

7. Qu'est-ce qui est pareil?

8. Qu'est-ce qui est différent?

9. Se comportait-il de cette manière avant son entrée à l'école?

10. Y a-t-il autre chose que vous aimeriez nous dire au sujet des comportements violents de votre enfant envers ses pairs à l'école?

\section{PARTIE 2}

\section{DÉCRIRE LA PROBLÉMATIQUE DES PARENTS D'ENFANTS DE 3 À 9 ANS AYANT DES COMPORTEMENTS VIOLENTS ENVERS LEURS PAIRS AU PRÉSCOLAIRE ET AU PRIMAIRE}

11. Selon vous, pourquoi cela ne va pas à l'école?

Est-ce en raison de:

$\square$ séparation ou divorce

$\square$ perte d'emploi d'un parent

$\square$ problèmes de santé

$\square$ relations difficiles au sein de la famille (avec ses frères et sœurs, ses beaux-parents)

$\square$ relations difficiles entre votre enfant et son enseignant(e) $\square$ relations difficiles entre votre enfant et ses pairs

$\square$ naissance d'un autre enfant

$\square$ déménagement(s)

$\square$ autre

12. Pouvez-vous nous dire autre chose sur votre situation familiale? 


\section{PARTIE 3}

\section{EXPLORER LES PRATIQUES ÉDUCATIVES PARENTALES} AYANT UN EFFET POSITIF ET NÉGATIF SUR LES COMPORTEMENTS VIOLENTS DE LEUR ENFANT

13. Si vous avez répondu que votre enfant a des comportements violents à la maison, que faites-vous quand ça se produit? Donnez un exemple.

14. Si vous avez répondu que votre enfant n'a pas de comportements violents à la maison, que faites-vous pour que ça marche bien? Donnez un exemple.

15. Quelles sont vos pratiques éducatives qui ont un effet positif sur les comportements violents de votre enfant?

16. Inversement, quelles sont vos pratiques éducatives qui ont un effet négatif sur les comportements violents de votre enfant?

17. Est-ce que cela vous arrive de ne rien faire parce que vous êtes fatigué ou pour une autre raison?

18. Faites-vous quelque chose seulement lorsque vous êtes de mauvaise humeur ou à bout?

19. Pouvez-vous nous dire autre chose sur les pratiques éducatives que vous utilisez ou avez utilisées jusqu'à maintenant (manières de faire) lorsque votre enfant a des comportements violents à la maison? 


\section{PARTIE 4}

\section{TRACER LA TRAJECTOIRE DE LA COLLABORATION ENTRE L'ÉCOLE ET LES PARENTS, AINSI QUE LES FACTEURS DE SOUTIEN ET LES OBSTACLES SOUS-JACENTS}

20. L'école communique-t-elle ou a-t-elle déjà communiqué avec vous pour vous faire part des bons comportements de votre enfant?

$\square$ oui

$\square 1$ fois par semaine

$\square$ plusieurs fois par semaine

$\square 1$ fois par mois

$\square$ plusieurs fois par mois

$\square 1$ fois dans l'étape

$\square$ autre

$\square$ non

21. L'école communique-t-elle ou a-t-elle déjà communiqué avec vous pour vous faire part des comportements violents de votre enfant?

$\square$ oui

$\square 1$ fois par semaine

$\square$ plusieurs fois par semaine

$\square 1$ fois par mois

$\square$ plusieurs fois par mois

$\square 1$ fois dans l'étape

$\square$ autre

$\square$ non

22. De quelle manière cela s'est-il passé?

23. Pensez-vous que l'école connaît et comprend votre situation familiale?

24. Si oui, percevez-vous que l'école réalise l'impact de votre situation sur votre vie familiale?

25. Percevez-vous que l'école reconnaît vos qualités en tant que parent? 
26. Qu'avez-vous déjà fait pour qu'il y ait une collaboration avec l'école?

27. Pouvez-vous nous dire autre chose sur la collaboration avec l'école?

\section{PARTIE 5}

CERNER les PERCEPTIONS DES PARENTS AU SUJET DE L'INFLUENCE, VOIRE LE CONTRÔLE, QU'ILS EXERCENT SUR LA SITUATION-PROBLÈME DE LEUR ENFANT À L'ÉCOLE

28. Avant d'arriver à l'étape du plan d'enseignement individualisé (PEI), l'école vous a-t-elle consulté sur la manière d'intervenir auprès de votre enfant?

29. L'école vous a-t-elle invité à participer à la recherche d'une solution?

30. L'école vous a-t-elle invité à participer aux décisions à prendre concernant votre enfant?

31. Avez-vous eu une influence sur les décisions déjà prises par l'école concernant votre enfant? Donnez un exemple.

32. Avant de suspendre ou d'envisager la possibilité de suspendre votre enfant à l'externe, l'école vous a-t-elle demandé votre avis?

$\square$ oui $\quad \square$ non $\quad \square$ plus ou moins

33. Si oui, l'école a-t-elle tenu compte de votre avis?

$\square$ oui $\square$ non $\square$ plus ou moins

34. Comment cela s'est-il passé? 


\section{PARTIE 6}

DÉGAGER LES OCCASIONS PERMETTANT AUX PARENTS DE POSER DES GESTES D'EMPOWERMENT POUR AMÉLIORER LEUR SITUATION FAMILIALE ET LA SITUATION-PROBLĖME DE LEUR ENFANT À L'ÉCOLE

35. Pouvez-vous identifier des gestes concrets que vous avez posés qui ont permis d'améliorer votre situation familiale ou celle de votre enfant à l'école?

36. Connaissez-vous d'autres parents qui ont un enfant ayant des comportements violents avec qui vous pouvez en parler?

$\square$ oui $\square$ non $\square$ plus ou moins

37. Si oui, parmi ces parents, y en a-t-il dont l'enfant fréquente la même école que le vôtre?

38. Qu'est-ce que ces échanges vous apportent?

39. Y a-t-il d'autres personnes ou des professionnels avec qui vous pouvez parler de votre situation?

40. Y a-t-il d'autres choses qui permettraient d'améliorer votre situation et celle de votre enfant à l'école? 


\section{PARTIE 7}

CERNER LES PERCEPTIONS DES PARENTS AU SUJET DE LA SUSPENSION SCOLAIRE ET DES AUTRES MESURES DISCIPLINAIRES APPLIQUÉES PAR L'ÉCOLE EN RÉPONSE AUX COMPORTEMENTS VIOLENTS DE LEUR ENFANT ENVERS SES PAIRS AU PRÉSCOLAIRE ET AU PRIMAIRE

41. Lorsque votre enfant a des comportements violents à l'école, de quelle manière l'école intervient-elle ou est-elle intervenue?
$\square$ avertissement verbal système de jetons
$\square$ nom au tableau
$\square$ perte de privilèges
$\square$ nom au fichier
$\square$ retenue pendant la récréation
$\square$ isolement dans la classe (time $\square$ retenue après la classe out)
$\square$ isolement lors de la période du dîner
$\square$ devoir supplémentaire
$\square$ note dans l'agenda scolaire
$\square$ fiche de réflexion
$\square$ rappel du code de conduite
$\square$ fiche de comportement
$\square$ note dans le bulletin
$\square$ conseil de coopération (cercle magique)
$\square$ appel téléphonique aux parents
$\square$ retrait de la classe
$\square$ rencontre à l'école
$\square$ visite au bureau de la $\square$ suspension scolaire (interne ) direction $\square$ suspension scolaire (externe)
$\square$ autre__

42. Pensez-vous que la suspension ou la possibilité de suspension scolaire externe peut aider votre enfant?

43. Y a-t-il ou y a-t-il eu des interventions qui s'adressaient à votre enfant que vous avez appréciées de la part... et pourquoi ? de l'enseignant? de la direction d'école? du travailleur social? autre (préciser)? 
44. Inversement, y a-t-il ou y a-t-il eu des interventions qui s'adressaient à votre enfant que vous n'avez pas appréciées de la part... et pourquoi?

de l'enseignant?

de la direction d'école?

du travailleur social?

autre (préciser)?

45. Pouvez-vous nous dire autre chose sur la manière d'intervenir de l'école?

\section{PARTIE 8}

\section{DONNÉES SOCIODÉMOGRAPHIQUES}

46. Sexe de l'enfant

garçon

$\square$ fille

47. Répondant pour l'entrevue

48. Statut familial

$\square$ Père et mère

$\square$ Mère biologique

(famille monoparentale)

Fréquence des visites chez le père

$\square$ Père biologique (famille monoparentale)

Fréquence des visites chez la mère

$\square$ Famille reconstituée de la mère

Fréquence des visites chez le père

Fréquence des visites chez la mère du père

Fréquence des visites chez le père de la mère

Fréquence des visites chez la mère du père

Liens avec la mère Liens avec le père 
Tuteurs

Famille d'accueil

Autre

49. Âge des parents

$\square$ moins de 20 ans

$\square$ entre 20 et 24

$\square$ entre 25 et 29

$\square$ entre 30 et 34

$\square$ entre 35 et 39

50. Revenu familial annuel

$\square$ moins de $20000 \$$

$\square$ entre $20000 \$$ et $24999 \$$

$\square$ entre $25000 \$$ et $29999 \$$

$\square$ entre 30000 \$ et $34999 \$$

$\square$ entre $35000 \$$ et $39999 \$$

51. Occupation de la mère

$\square$ à la maison

$\square$ travailleuse

$\square$ chômeuse

52. Niveau de scolarité de la mère

$\square$ élémentaire

$\square$ secondaire

53. Occupation du père

$\square$ à la maison

$\square$ travailleur

$\square$ chômeur
Liens avec la mère Liens avec le père Liens avec la mère Liens avec le père

$\square$ entre 40 et 44

$\square$ entre 45 et 49

$\square$ entre 50 et 54

$\square 55$ et plus $\square$ entre 40000 \$ et 44999 \$

$\square$ entre $45000 \$$ et $49999 \$$

$\square$ entre $50000 \$$ et $59999 \$$

$\square$ entre $60000 \$$ et $74999 \$$

$\square 75000$ \$ et plus $\square$ prestation de derniers recours

$\square$ autre

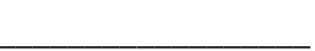


54. Niveau de scolarité du père

$\square$ élémentaire

$\square$ secondaire

55. Origine ethnique de la mère

canadienne-française

56. Origine ethnique du père

canadienne-française

57. Langue parlée à la maison

$\square$ français

$\square$ anglais $\square$ collège

université

autre

autre

$\square$ autre

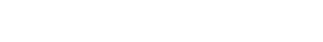

58. Nombre d'enfants dans la famille autres que l'enfant concerné

$\square$ frères

$\square$ sœurs

$\square$ aucun

59. Âge des enfants de la famille

$\square$ moins de 5 ans

$\square$ entre 5 et 9 ans

$\square$ entre 10 et 14 ans $\square$ demi-frères

$\square$ demi-sœurs

$\square$ entre 15 et 19 ans

$\square$ entre 20 et 24 ans

$\square$ plus de 25 ans

60. Âge de l'enfant concerné

$\square$ 3-4 ans

4-5 ans

5-6 ans $\square$ 6-7 ans

$7-8$ ans

8-9 ans

61. Rang occupé dans la famille

$\square$ aîné

entre et sur $\square$ unique (nombre total d'enfants)

$\square$ cadet 
62. Niveau scolaire

$\square$ préscolaire (garderie)

$\square 2^{\mathrm{e}}$ année

$\square$ jardin

$\square 3^{\mathrm{e}}$ année

$\square$ maternelle

$\square$ autre

$\square 1^{\text {re }}$ année

63. Antécédent scolaire

$\square$ dépistage précoce et continu

$\square$ suivi par un travailleur social

$\square$ difficultés d'apprentissage scolaire

$\square$ suivi par un psychologue privé

$\square$ PEI

$\square$ école ou classe spéciales (placement en Section 20)

$\square$ suivi par un psychologue scolaire

$\square$ référé au Centre psychosocial

$\square$ doublement et redoublement

$\square$ autre suivi

$\square$ suivi par un enseignantressource

64. Antécédent médical

$\square$ problème de santé

$\square$ handicap

$\square$ médication

$\square$ autre

65. Autres renseignements pertinents 



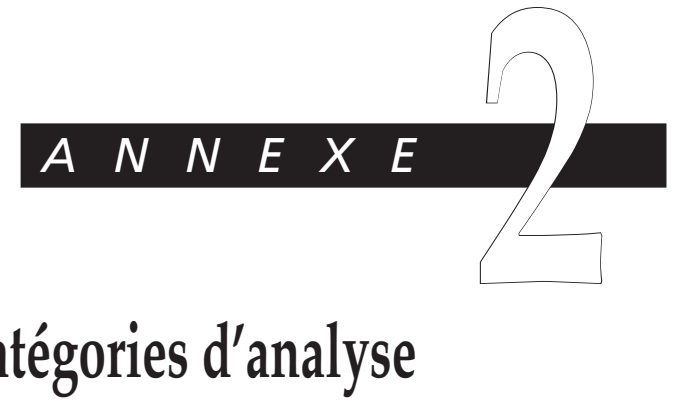

A. L'ENFANT

B. LES PARENTS ET LA FAMILLE

C. L'ÉCOLE 



\section{A. L'ENFANT}

\section{CARActéristiques PERSONNELles}

\section{FACTEURS DÉVELOPPEMENTAUX (Gagnon et Vitaro, 2000)}

\subsection{Tempérament}

Problèmes externalisés

Grognon, agité, impulsif, obstiné, immature, influençable. Tendance à mentir, ne pas écouter, à être agressif, colérique (crises), impatient, d'humeur variable, irritable, imprévisible, frustré, etc.

\section{Problèmes internalisés}

Anxieux, dépressif, renfrogné, méfiant, réservé, introverti, etc.

Attitudes et traits positifs

Heureux, démonstratif, sociable, calme, mature, curieux, confiant, honnête, sensible, s'affirme, fait preuve de leadership, a le sens de la justice, aime faire rire (clown), est intelligent, appliqué, etc.

\subsection{Maturation physique et sexuelle}

Description des caractéristiques et évolution dans le temps.

\section{COMPORTEMENTS DE L'ENFANT (Gagnon et Vitaro, 2000)}

\subsection{Opposition à l'adulte}

Cherche à provoquer, désobéit, cherche à avoir le dernier mot (réplique), conteste, etc.

\subsection{Besoins particuliers}

Besoin d'attention, de sécurité, de s'identifier à ses pairs, etc.

\subsection{Estime de soi/confiance en soi}

\subsection{Relations dans la fratrie}

\subsection{Relations avec les pairs}

\subsection{Comportements à l'école}

Attention/Concentration avec ou sans hyperactivité

Difficultés à se concentrer, tendance à oublier ou perdre des objets ou, à l'inverse, bonne capacité de concentration, etc. 


\begin{abstract}
Apprentissage
Difficulté d'apprentissage, trouble du langage, rendement scolaire, perception du parent du rendement scolaire, raison qui diminue les difficultés d'apprentissage (p. ex.: Plan d'enseignement individualisé (PEI), encadrement, aide individuelle), etc.

Attitudes face à l'école

Favorables / défavorables, intérêt / désintérêt face au processus de scolarisation, motivation, etc.
\end{abstract}

\title{
2.7. Autres comportements
}

Idées suicidaires, vol, fugue, etc.

\section{TYPES DE COMPORTEMENTS VIOLENTS (Straus et Gelles, 1990)}

\section{VIOLENCE VERBALE SANS GESTES}

Insulte, blâme, intimide, menace de frapper ou de lancer des choses, etc.

\section{VIOLENCE VERBALE AVEC GESTES}

Détruit ses choses ou de celles des autres, lance, casse ou donne des coups de pied sur des objets (devant les autres), crache, etc.

\section{VIOLENCE PHYSIQUE}

Malmène (pousse, secoue ou bouscule), lance ou casse des objets (à la personne), frappe (avec ou sans objet), mord, donne des coups de pied, donne des coups de poing, se bat, utilise une arme blanche ou un objet contondant, brûle autrui, pince, etc.

\section{HISTORIQUE DES COMPORTEMENTS VIOLENTS}

\section{FRÉQUENCE ET LIEUX DES COMPORTEMENTS VIOLENTS}

\subsection{Fréquence}

Quotidienne, hebdomadaire (un épisode, plusieurs épisodes), mensuelle (un épisode, plusieurs épisodes), saisonnière (un épisode, plusieurs épisodes), isolée, autre, etc. 


\subsection{Lieux où surviennent les comportements violents}

À la maison, à l'école, dans l'entourage (différents et similaires).

\section{TRAJECTOIRE DES COMPORTEMENTS VIOLENTS}

\subsection{Période précédant l'entrée scolaire}

Présence ou absence de comportements violents.

\subsection{Présence des comportements violents}

À la maison (famille qui n'envoie pas son enfant à la garderie ou période précédant l'entrée à la garderie), à la garderie, à la maternelle, au jardin, en première année, en deuxième année, en troisième année et plus.

\section{RAISONS OU CAUSES EXPLIQUANT LA PRÉSENCE DES COMPORTEMENTS VIOLENTS SELON LE PARENT}

\section{RAISONS OU CAUSES SE RAPPORTANT À L'ENFANT}

Mauvaise gestion du stress, dépression, handicap physique (p. ex.: surdité), changements, besoin d'aide individuelle, médication, racisme.

Se rapportant aux «compétences sociales »: problèmes de socialisation, relations difficiles avec les pairs, se faire accepter par les pairs, approche de festivités, peur du rejet, etc. Se rapportant à ses fréquentations (pairs qui ont une mauvaise influence, fréquentation d'enfants plus âgés, violence ou provocation de la part des pairs, défense d'un ami, etc.

\section{RAISONS OU CAUSES SE RAPPORTANT À LA FAMILLE}

Fréquence et type de contacts avec les parents: Famille monoparentale, reconstituée ou recomposée, nouveau conjoint de l'un des parents, difficultés financières, parent(s) qui travaille(nt) le soir, absence d'un modèle masculin, parent(s) agressif(s), décès, dépression du parent, rejet de l'enfant par la famille, ennui du parent séparé, visites de l'enfant chez le parent séparé, conflits entre les parents, relations difficiles avec les parents et/ou la fratrie, favoritisme au sein de la famille, parent qui manque de temps pour répondre aux besoins de l'enfant, etc. 


\section{RAISONS OU CAUSES SE RAPPORTANT À L'ÉCOLE}

N'aime pas l'école, mauvaise gestion de classe par l'enseignant, absences répétées de l'enseignant, grand groupe, somme et niveau de difficulté des devoirs, changements d'école, valeurs de l'école qui diffèrent des valeurs familiales, changement d'enseignant, changement de direction, relations difficiles avec l'enseignant, etc.

\section{AUTRES RAISONS OU CAUSES}

Influence de la télévision, des jeux vidéo, abus verbal, physique ou sexuel, etc.

\section{RAISONS OU CAUSES EXPLIQUANT LA DIMINUTION DES COMPORTEMENTS VIOLENTS SELON LE PARENT}

\section{RAISONS OU CAUSES SE RAPPORTANT À L'ENFANT}

Médication: Nouvelle ou augmentation de la posologie, inscription à une activité récréative, aller au lit plus tôt, etc.

\section{RAISONS OU CAUSES SE RAPPORTANT À LA FAMILLE}

Voit son père plus régulièrement, séparation des parents, etc.

\section{RAISONS OU CAUSES SE RAPPORTANT À L'ÉCOLE}

Changement d'enseignant, pupitre individuel, classe spéciale, relation de confiance avec le personnel scolaire, valorisation de l'enfant, période de sommeil dans l'après-midi (école), etc.

\section{AUTRES RAISONS OU CAUSES}

Impact du suivi professionnel, etc.

\section{DONNÉES SOCIODÉMOGRAPHIQUES DE L'ENFANT}

\section{DONNÉES GÉNÉRALES}

\subsection{Sexe}

1.2. Âge de l'enfant concerné
9-10 ans

Autre 
1.3. Rang occupé dans la famille

$\square$ Aîné

$\square$ Cadet

$\square$ Unique

$\square$ Benjamin

1.4. Niveau préscolaire et scolaire

$\square$ Garderie

$\square$ Deuxième année

$\square$ Maternelle

$\square$ Troisième année

$\square$ Jardin

$\square$ Autre

$\square$ Première année

1.5. Origine ethnique de l'enfant

$\square$ Canadienne-française

$\square$ Française

$\square$ Canadienne-anglaise

$\square$ Chinoise

$\square$ Haïtienne

$\square$ Finlandaise

Africaine

Marocaine

$\square$ Espagnole

$\square$ Salvadorienne

\section{ANTÉCÉDENTS SCOLAIRES}

2.1. Dépistage précoce et continu

2.2. Référé au Centre psychosocial

2.3. Suivi par un professionnel

$\square$ Enseignant-ressource

$\square$ Pédiatre

$\square$ Éducateur

$\square$ Pédopsychiatre

$\square$ Travailleur social

$\square$ Stagiaire

$\square$ Psychologue privé

$\square$ Psychoéducateur

$\square$ Psychologue scolaire

$\square$ Médiateur

$\square$ Orthopédagogue

$\square$ Étudiant

$\square$ Orthothérapeute

$\square$ Autre

Ergothérapeute

2.4. Doublement et redoublement

2.5. Plan d'enseignement individualisé (PEI)

2.6. Section 20 (Placement dans une école ou classe spéciales)

2.7. Autre 


\section{ANTÉCÉDENTS MÉDICAUX}

\subsection{Problème de santé et handicaps}

Séquelles de la prématurité

$\square$ Allergies

$\square$ Asthme

$\square$ Maniaco-dépression

$\square$ Problèmes neurologiques

$\square$ Problèmes physiques de nature psychosomatique

$\square$ Syndrome Gilles de la Tourette

$\square$ Autre

\subsection{Médication}

$\square$ Ritalin $^{\circledR}$

$\square$ Anti-dépresseurs

$\square$ Ventalin ${ }^{\circledR} /$ Flovent $^{\circledR}$ (Asthme)

3.3. Troubles divers: DSM-IV (APA, 1996)

Troubles du langage

$\square$ Dyslexie

$\square$ Orthophonie

Difficultés d'apprentissage

$\square$ Légères

Troubles de comportement

$$
\text { Légers }
$$

Motricité

Fine $\square$ Développement langagier

Autre

$\square$ Sévères
Épipen (allergies sévères)

Autre 


\section{B. LES PARENTS ET LA FAMILLE}

\section{Problématique des parents et de la situation familiale}

\section{DIFFICUlTÉS RELIÉES AU COUPLE}

Séparation: Temporaire, multiples, permanente, violence conjugale, etc. Divorce.

\section{DIFFICULTÉS RELIÉES AU CONTEXTE FAMILIAL}

Famille monoparentale, reconstituée ou recomposée, relations familiales tendues, dysfonctionnements intrafamiliaux ou problèmes de gestion familiale, etc.

3. DIFFICULTÉS RELIÉES À LA RELATION ET À LA DISPONIBILITÉ AVEC L'ENFANT

Déresponsabilisation envers l'enfant, manque de temps pour répondre aux besoins familiaux, naissance d'un autre enfant, adoption (type), mesure familiale et/ou mesure de protection légale, etc.

4. DIFFICULTÉS RELIÉES À LA SITUATION ÉCONOMIQUE ET PROFESSIONNELLE DES PARENTS

Sans emploi, en recherche d'emploi, perte d'emploi, etc.

5. PROBLÈMES DE SANTÉ

\subsection{Santé mentale}

5.2. Santé physique

6. DÉMÉNAGEMENTS

7. DÉCÈS

Parents, grands-parents, proches parents, amis, fratrie, etc. 


\section{Compétences éducatives parentales (Trudelle et Montambault, 1994; TREMblay et al., 1996)}

\section{ATTITUDES AYANT UN IMPACT POSITIF}

Cohérence parentale (similarités entre ce qui est dit et ce qui est fait), consensus parental (les parents s'entendent entre eux), expressions affectueuses nombreuses, écoute attentive, respect du rythme de l'enfant, compréhension de l'enfant, ouverture, empathie, valorisation des comportements prosociaux, etc.

\section{ATTITUDES AYANT UN IMPACT NÉGATIF}

Incohérence parentale (différence entre ce qui est dit et ce qui est fait), manque ou absence de consensus parental, manque dans la manifestation de marques d'affection, perceptions et/ou attributions négatives des comportements de l'enfant (p. ex.: l'enfant agit ainsi car il ne veut pas: s'améliorer, écouter, changer), surprotection, modèle parental inadéquat (fais ce que je dis, pas ce que je fais), fermeture, antipathie, dévalorisation des comportements prosociaux, etc.

\section{PRATIQUES AYANT UN IMPACT POSITIF (Vézina et al., 1995)}

Présence de discipline et d'encadrement, constance dans l'application de la routine et dans les interventions, contrôle de soi avant l'intervention, présence de limites efficaces par rapport aux comportements violents, disputes des parents en l'absence de l'enfant, interventions face aux comportements inadéquats, activités parents/enfant, encouragements, discussions franches et ouvertes, utilisation de l'humour, renforcement des comportements adéquats, reprise de l'enfant seul et non devant autrui, technique 1-2-3, perte de privilèges, présence de routines, explications brèves et concrètes, ignorance des comportements inadéquats, environnement calme, contacts physiques (toucher), tonalité acceptable de la voix, distractions, périodes de réflexion, inscription à des activités récréatives, présence d'animaux, périodes de répit, utilisation du jeu, enseignement à la maison, contact visuel, enseignement de comportements prosociaux, sensibilisation aux conséquences de ses actes, donner des responsabilités (tâches d'écriture), instrument de défoulement (batterie, punching bag), utilisation de stratégies démocratiques, etc. 


\section{PRATIQUES AYANT UN IMPACT NÉGATIF (Vézina et al., 1995)}

Inconstance dans l'application de la discipline et de l'encadrement (parfois intervient, parfois n'intervient pas), absence de discipline et d'encadrement, perte de contrôle de soi (hausser la voix ou crier), inconstance dans l'imposition de limites efficaces par rapport aux comportements violents, échange de nature coercitive et punitive, conséquences disproportionnées par rapport aux actions posées, disputes des parents devant l'enfant, renforcement des comportements non adéquats, inconsistance dans l'application de la routine (parfois technique 1-2-3, parfois pertes de privilèges), absence de routine, dénigrement de l'enfant, ne permet pas à l'enfant de faire des choix, consignes longues et abstraites, ignorance de l'enfant, environnement bruyant, utilisation de la force physique, absence de fermeté dans le ton de la voix, présence de consignes autoritaires, utilisation de stratégies permissives ou de laisser-faire, plusieurs essais répétés sans succès, etc.

\section{EMPOWERMENT PARENTAL (ADAMS, 1996; Cameron et Cadell, 1997; Le Bossé, 1996; RAPPAPORT, 1987; VINCENT, 1996)}

\section{PERCEPTION ET SENTIMENTS DES PARENTS D'EXERCER DE L'INFLUENCE, VOIRE DU CONTRÔLE, À LA MAISON}

\subsection{Vit des réussites}

Maîtrise de soi (contrôle interne), sentiment de compétence (efficacité personnelle), le lien parent/enfant est rétabli (résolutions de la situation-problème), réponses positives de l'enfant aux demandes du parent, le parent a un sentiment de contrôle sur la situation familiale, le parent a plus de temps de qualité à consacrer à l'enfant, diminution ou cessation des comportements violents.

\subsection{Vit des échecs}

Perte de contrôle de soi (interne), sentiment d'incompétence, le lien parent/enfant est rompu, réponses négatives de l'enfant face aux demandes du parent, perte de contrôle sur la situation familiale, le parent vit de l'épuisement, sentiment d'impuissance, sentiment de culpabilité, volonté de procéder au placement de l'enfant, maintien des comportements 
violents, augmentation de la fréquence ou de l'amplitude des comportements violents, désobéissance, augmentation des interventions coercitives par le parent.

\subsection{Ne sait pas}

\section{PERCEPTION ET SENTIMENTS DES PARENTS D'EXERCER} DE L'INFLUENCE, VOIRE DU CONTRÔLE, À L'ÉCOLE

\subsection{Vit des réussites}

Maîtrise de soi (contrôle interne), sentiment de compétence (efficacité personnelle), le parent a un sentiment de contrôle sur la situation scolaire, exerce de l'influence sur les décisions concernant l'enfant.

\subsection{Vit des échecs}

Perte de contrôle de soi (interne), sentiment d'incompétence, perte d'emprise sur la situation scolaire, sentiment d'impuissance, sentiment de culpabilité, n'exerce pas d'influence sur les décisions concernant l'enfant.

\subsection{Ne sait pas}

\section{GESTES D'EMPOWERMENT DES PARENTS}

DANS LA FAMILLE (Breton, 1998; Pinderhughes, 1995)

\subsection{Prise de conscience}

S'interroge sur la situation-problème de l'enfant, sur ses capacités parentales et familiales.

3.2. Prise de décisions concernant sa situation familiale (actions concrètes)

Cesse de travailler, demande du répit, obtient un emploi stable.

3.3. Pistes de solutions apportées par le parent pour améliorer sa situation familiale future

\section{GESTES D'EMPOWERMENT DES PARENTS À L'ÉCOLE} (Breton, 1998; Pinderhughes, 1995)

\subsection{Prend sa place et s'affirme}

Discute, participe aux rencontres, donne son point de vue, dit le fond de sa pensée, etc. 


\subsection{Propose des solutions}

Besoins individuels (pupitre individuel, diminution de la somme des devoirs, période de repos en après-midi, surveillance accrue aux heures de récréation, petit groupe d'élèves, changement de classe, rencontre le soir, stage pratique, placement en Section 20, méthodes d'interventions (technique 1-2-3, renforcement positif, jeu, accessoires de défoulement, projet avec l'université, etc.), sources de motivation (piano, club d'échecs), soutien à l'enseignement (le parent offre d'enseigner à la maison et d'aider en classe), gestion scolaire (évaluation de l'enseignant par les élèves, remplaçant régulier), etc.

\subsection{Prend des mesures, des actions concrètes, des initiatives}

S'oppose aux décisions déjà prises par l'école, demande des explications, convoque ou exige une rencontre, porte plainte, dénonce la situation, fait des menaces, utilise un tiers parti (enfant, parent ou professionnel), cite des articles de loi, retire l'enfant de l'école (déménagement, changement de conseil scolaire), fait l'école à la maison, etc.

\subsection{Résistance (Ashforth et Mael, 1998; Rooney, 1992)}

Change de sujet durant une discussion, menace de recourir à un avocat, refuse de mettre en application les conseils reçus, refuse de s'impliquer dans la situation-problème de l'enfant à l'école, refuse de donner son opinion, refuse de demander de l'aide, refuse l'aide proposée, refuse de parler de sa situation familiale, met un terme à la collaboration (quitte une rencontre), s'abstient de contrarier le personnel scolaire, absence de geste de collaboration avec l'école.

\subsection{Adaptation (Zimmerman, 1995)}

Mobilise des ressources formelles, informelles et communautaires afin d'améliorer ses conditions de vie.

\subsection{Protection et survie (Dubet et Martuccelli, 1996; Vatz-Laaroussi, 1996)}

\section{SUIVIS PROFESSIONNELS (Zimmerman, 1995)}

\subsection{Demande d'aide et description des suivis}

Demande d'aide professionnelle, suivi auprès d'un professionnel (travailleur social, psychologue, pédopsychiatre, pédiatre, médecin, enseignant-ressource, etc.), application des conseils reçus par un professionnel, données sociodémographiques: référé, suivi, PEI, etc. 
5.2. Perception des autres professionnels et impacts positifs et négatifs des suivis

Du travailleur social, du psychologue, du pédopsychiatre, du pédiatre, du médecin, de l'enseignant-ressource, etc.

6. RÉSEAUX (Zimmerman, 1995)

6.1. Réseau informel et impacts positifs et négatifs

Échanges avec la famille, le voisinage, démarches pour obtenir de l'information auprès des amis, du voisinage et de la famille. Parents qui n'ont pas de contacts avec des membres du réseau informel et qui en voudraient.

6.2. Réseau formel et impacts positifs et négatifs

Échanges avec des membres d'un groupe de soutien ou d'un organisme communautaire, démarches pour obtenir de l'information auprès des membres du réseau formel.

\section{COMPRÉHENSION-CRITIQUE DU PARENT}

\section{(Pinderhughes, 1995)}

\subsection{Externe à la situation familiale}

Capacité d'analyser la situation familiale, définition de comportement violent qui diffère de celle de l'école, seuil de tolérance différent face aux comportements inadéquats de l'enfant, etc.

\subsection{Face à l'école que fréquente l'enfant}

Capacité d'analyser la situation à l'école, manque de communication entre le personnel scolaire, etc.

8. LUTTE COLLECTIVE (Breton, 1994; Gutiérrez, 1994; Lee, 1994; Moreau et Léonard, 1993)

Actions concrètes collectives visant un changement social.

\section{DONNÉES SOCIODÉMOGRAPHIQUES DES PARENTS}

\section{NIVEAU DE SCOLARITÉ}

\subsection{Mère}

$\square$ Élémentaire (ou primaire) $\square$ Collège (ou cégep)

$\square$ Secondaire $\quad \square$ Université 
1.2. Père

$\square$ Élémentaire (ou primaire) $\square$ Collège (ou cégep)

$\square$ Secondaire $\quad \square$ Université

2. MILIEU SOCIOÉCONOMIQUE (REVENU FAMILIAL ANNUEL)

2.1. Sous le seuil de la pauvreté $\square$ Moins de $19999 \$$

2.2. Classe

$\square$ Entre $20000 \$$ et $24999 \$ \quad \square$ Entre $30000 \$$ et $34999 \$$

$\square$ Entre $25000 \$$ et $29999 \$$

2.3. Classe moyenne

$\square$ Entre $35000 \$$ et $39999 \$ \quad \square$ Entre $45000 \$$ et $49999 \$$

$\square$ Entre $40000 \$$ et $44999 \$$

2.4. Classe moyenne-supérieure

$\square$ Entre $50000 \$$ et $59999 \$ \quad \square$ Entre $70000 \$$ et $74999 \$$

$\square$ Entre $60000 \$$ et $69999 \$$

2.5. Classe supérieure

Plus de $75000 \$$

2.6. Non disponible

3. STATUT

\subsection{Civil}

$\square$ Marié

$\square$ Divorcé

$\square$ Conjoint de fait

$\square$ Célibataire

$\square$ Séparé

\subsection{Familial}

$\square$ Monoparental

$\square$ Reconstitué de la mère

$\square$ Reconstitué du père

$\square$ Recomposé de la mère

$\square$ Recomposé du père

$\square$ Autre

\subsection{Décès d'un parent}

$\square$ Veuve

Veuf

\subsection{Orientation sexuelle}

Hétérosexuel

Bisexuel

Homosexuel 


\section{ORIGINE ETHNIQUE}

\section{5. ÂGE DES PARENTS}

\subsection{Mère:}

$\square$ Moins de 20 ans

$\square$ Entre 20 et 24 ans

$\square$ Entre 25 et 29 ans

$\square$ Entre 30 et 34 ans

$\square$ Entre 35 et 39 ans

\subsection{Père}

Moins de 20 ans

$\square$ Entre 20 et 24 ans

$\square$ Entre 25 et 29 ans

$\square$ Entre 30 et 34 ans

$\square$ Entre 35 et 39 ans $\square$ Entre 40 et 44 ans

$\square$ Entre 45 et 49 ans

$\square$ Entre 50 et 54 ans

$\square 9$ ans et plus

$\square$ Entre 40 et 44 ans

$\square$ Entre 45 et 49 ans

$\square$ Entre 50 et 54 ans

$\square 9$ ans et plus

\section{PARENTS}

$\square$ Mère

Mère adoptive

$\square$ Père adoptif

Père

$\square$ Grand-mère

$\square$ Grand-père

\section{LANGUE PARLÉE À LA MAISON}

Français

Anglais
Autre

Préciser: 


\section{L'ÉCOLE}

\section{INFORMATION TRANSMISE AUX PARENTS}

\section{TYPE D'INFORMATION TRANSMISE}

Bons et mauvais comportements de l'enfant, état de santé, oubli fait par l'enfant, rendement scolaire, etc.

2. MODE ET FRÉQUENCE DE TRANSMISSION DE L'INFORMATION

3. PERCEPTIONS DES PARENTS AU SUJET DE L'INFORMATION TRANSMISE PAR L'ÉCOLE CONCERNANT LES COMPORTEMENTS VIOLENTS DE L'ENFANT

En accord ou en désaccord par rapport à l'information transmise par l'école concernant les comportements violents de l'enfant, jugements et qualificatifs attribués par les parents au sujet de ces échanges, avec qui le parent communique davantage ou moins, comparaison de la qualité des échanges d'information avec les années précédentes ou entre les membres du personnel scolaire (en roulement ou non) versus le parent, personne qui communique avec le parent, etc.

\section{ATtITUDES, GESTES ET ACTIONS CONCRÈTES POSÉS} PAR L'ÉCOLE ET PAR LE PERSONNEL SCOLAIRE ENVERS LE PARENT (EPSTEIN, 1995)

\section{DE COLLABORATION}

1.1. Invitation à participer aux réunions, aux décisions, aux activités, aux tâches et aux responsabilités scolaires

1.2. Incitation à donner son opinion, à proposer des solutions, à participer aux décisions

Concernant la suspension scolaire interne et externe, le placement en Section 20, le Plan d'enseignement individualisé (PEI), la discipline et l'encadrement scolaires, la médication, etc.

1.3. Proposition de solutions aux parents

1.4. Invitation à s'impliquer en milieu scolaire et/ou familial 


\subsection{Donne des outils aux parents}

Livres, documentation, techniques, etc.

1.6. Prend une entente avec le parent

1.7. Apporte du soutien aux parents

\section{DE NON-COLLABORATION}

Harcèlement, imposition, refuse que le parent participe aux comités, aux réunions, pas de retours d'appels, opposition, prend des décisions sans consulter le parent. Tout ce qui est à l'opposé du point précédent (p. ex.: n'invite pas, n'incite pas, etc.).

\section{AtTITUDES, gESTES ET ACTIONS CONCRÈTES POSÉS PAR LE PARENT ENVERS L'ÉCOLE ET LE PERSONNEL SCOLAIRE (Christenson et SHERIDAN, 2001)}

\section{DE COLLABORATION}

\subsection{Démontre une ouverture}

Honnêteté, transparence, disponibilité, transmission d'attentes claires envers l'école, etc.

1.2. Participation aux réunions, aux décisions, aux activités, aux tâches et aux responsabilités scolaires, implication à l'école

1.3. Implication dans les décisions concernant l'enfant

1.4. Communique avec le personnel scolaire de sa propre initiative dans un objectif de collaboration

Par lettre, par téléphone, prend un rendez-vous, va porter l'enfant à l'école afin de rencontrer le personnel scolaire, demande une rencontre pour parler de son enfant, envoie de l'information écrite (évaluation, documentation, diagnostic, etc.), visites à l'école, etc.

\section{DE NON-COLLABORATION}




\section{Perceptions des parents envers l'École}

\section{AU SUJET DU RÔLE DU PERSONNEL SCOLAIRE (OU DU RÔLE QU'IL DEVRAIT JOUER)}

\subsection{Positives}

Sont considérés comme les experts, ils apportent de l'aide aux parents en lien avec la situation-problème de l'enfant, répondent aux attentes du parent, etc.

\subsection{Négatives}

Ils imposent leur expertise aux parents, abusent de leur pouvoir envers les parents, se déresponsabilisent de la situationproblème de l'enfant, ne répondent pas aux attentes du parent.

\section{AU SUJET DE LA COLLABORATION AVEC L'ÉCOLE}

Chaleureuse et supportante / absence de chaleur et de support, bonne et excellente / mauvaise et conflictuelle, suffisante et essentielle / insuffisante et non nécessaire, utile / inutile, volontaire / obligée, ouverte / fermée, etc.

\section{3. À L'ENDROIT DU PERSONNEL SCOLAIRE}

De l'enseignant, de la direction d'école, de la direction de la garderie scolaire, du surveillant, du conducteur d'autobus, etc.

\section{4. À L'ÉGARD DE L'INSTITUTION SCOLAIRE EN GÉNÉRAL}

\section{L'ÉCOLE PERÇUE PAR LES PARENTS}

\section{AU SUJET DE LA SITUATION FAMILIALE}

Compréhension / incompréhension familiale; respect / non-respect des attitudes parentales, respect / non-respect du rôle parental, présence d'écoute / absence d'écoute, etc.

\section{AU SUJET DU RÔLE DES PARENTS}

Sont considérés comme les experts, des partenaires, des applicateurs de tâches, se font consulter par devoir (loi), leur rôle n'est pas reconnu, etc. 


\section{L'ÉCOLE PERÇUE PAR L'ENFANT -}

\section{Perceptions du code de conduite}

1. COMPORTEMENTS VIOLENTS ENTRAÎNANT LA SUSPENSION OU LA POSSIBILITÉ DE SUSPENSION SCOLAIRE

2. APPLICATION DE LA SUSPENSION OU DE LA POSSIBILITÉ DE SUSPENSION SCOLAIRE (MÉO, 2001a)

Type de suspension scolaire: interne ou externe, nombre de jours de suspension, manière dont les événements se sont déroulés, exigences particulières de l'école au départ et au retour de la suspension scolaire, etc.

3. PERCEPTIONS DES PARENTS DES INTERVENTIONS DE L'ÉCOLE SUITE À DES INCIDENTS MINEURS

3.1. Retrait de la classe (bureau du directeur)

En accord, plus ou moins en accord, en désaccord, pas d'opinion
$\square$ Impacts positifs
Impacts négatifs

4. PERCEPTIONS DES PARENTS DES INTERVENTIONS DE L'ÉCOLE SUITE À DES INCIDENTS GRAVES (MÉO, 2000; MÉFO, 1994)

\subsection{Suspension scolaire interne}

En accord, plus ou moins en accord, en désaccord, pas d'opinion
Impacts positifs
Impacts négatifs

4.2. Suspension scolaire externe

En accord, plus ou moins en accord, en désaccord, pas d'opinion 


\section{Impacts positifs}

Absence de l'enfant perturbateur en classe, information du parent de l'agir de son enfant, interventions du parent auprès de son enfant, prise de conscience de l'enfant de la gravité de ses comportements, cessation des comportements, l'enfant vit de l'ennui à l'égard des pairs et de l'école, etc.

\section{Impacts négatifs}

Récompense de l'enfant: Congé scolaire, journée avec son parent, démotivation de l'enfant face à l'école (trajectoire vers le décrochage scolaire), diminution et/ou perte de l'estime de soi de l'enfant, transposition du problème scolaire en milieu familial, retard au niveau scolaire, accumulation de surplus de devoirs, situation davantage pénalisante pour le parent que pour l'enfant: absence du travail, surplus de tâches à la maison, démarche pour trouver une gardienne, conséquences disproportionnées face aux comportements, perte d'emploi, etc.

\subsection{Renvoi et placement en Section 20 (MÉO, 2001b)}

En accord, plus ou moins en accord, en désaccord, pas d'opinion
Impacts positifs
Impacts négatifs

\section{ALTERNATIVE PROPOSÉE PAR LE PARENT À LA SUSPENSION SCOLAIRE EXTERNE}

\subsection{Suspension interne}

\subsection{Rattrapage scolaire et activités de conscientisation}

\subsection{Autres}

Souhaits, suggestions, solutions ou actions concrètes; pistes de réflexions (philosophiques ou pratiques) touchant l'enfant, les autres enfants, le personnel scolaire ou l'école, les professionnels, l'entourage, la communauté, la société, etc. 



\section{Notices biographiques}

Marie DROLET, Ph. D., détient un doctorat en service social de l'Université Laval. Travailleuse sociale de carrière, elle est professeure, ainsi que responsable des études supérieures à l'École de service social de l'Université d'Ottawa. Elle y enseigne les problématiques touchant les enfants et les jeunes en difficulté, de même que l'intervention auprès des individus et des familles. Ayant toujours œuvré dans le champ de la prévention, ses derniers travaux concernent la collaboration entre les parents et les écoles, leurs démarches d'appropriation du pouvoir et de médiation avec divers milieux sociaux, ainsi que les changements au sein de la pratique sociale. Elle est chercheure sénior au Centre de recherche sur les services communautaires de l'Université d'Ottawa, membre individuel de l'Association canadienne des écoles de service social et membre de l'Ordre professionnel des travailleurs sociaux du Québec.

mdrolet@uottawa.ca

Maryse PAQUIN, Ph. D., détient un postdoctorat et un doctorat en éducation de l'Université du Québec à Trois-Rivières, conjointement avec l'Université du Québec à Montréal. Elle est professeure à la Faculté d'éducation de l'Université d'Ottawa. Son champ d'enseignement et de recherche est la didactique des sciences sociales. En relation avec ce champ, elle porte un intérêt particulier à la question de la prévention des comportements violents au préscolaire et au primaire dans les écoles francophones de la région d'Ottawa. Elle fait partie du Comité scientifique de la Conférence mondiale sur la violence à l'école et du comité de rédaction de la Revue internationale de la violence scolaire. Elle est membre fondatrice de l'Observatoire international de la violence à l'école et membre de l'Observatoire canadien de la prévention de la violence à l'école.

maryse.paquin@uottawa.ca 



\section{PARTICULARITÉS DES OUVRAGES DE LA COLLECTION ÉDUCATION-RECHERCHE}

La collection Éducation-Recherche présente les nouvelles orientations en éducation par le biais de résultats de recherche, et de réflexions théoriques et pratiques. Des outils de formation et d'intervention ainsi que des stratégies d'enseignement et d'apprentissage sont également présentés lorsqu'ils ont été validés, implantés et évalués dans le cadre de recherches. Les ouvrages à caractère scientifique doivent décrire une démarche rigoureuse de recherche et d'analyse ainsi que les résultats obtenus.

Afin d'assurer la rigueur scientifique des textes publiés, chacun d'eux est soumis à un processus d'arbitrage avec comité de lecture et évaluations externes. De plus, les délais de publication sont réduits au minimum afin de conserver l'actualité et l'à-propos des articles, des recherches et des études réalisés par les chercheurs et chercheures. Chaque texte est évalué par deux arbitres: un membre du comité de lecture de la collection et un spécialiste du domaine. Ces évaluations portent sur la pertinence du document et sur sa qualité scientifique (cohérence entre la problématique, les objectifs et la démarche méthodologique; profondeur des analyses; pertinence des conclusions...).

\section{Membres du comité de lecture}

Jean Archambault (Université de Montréal), Paul Boudreault (UQO), Jean-François Boutin (UQAR-Antenne de Lévis), Jacques Chevrier (UQO), Christine Couture (UQAC), Colette Deaudelin (Université de Sherbrooke), Godeliève Debeurme (Université de Sherbrooke), Serge Desgagné (Université Laval), Louise Dupuy-Walker (UQAM), Moussadak Ettayebi (Université Laval), Diane Gauthier (UQAC), Claude Genest (UQTR), Jacinthe Giroux (UQAM), Abdelkrim Hasni (Université de Sherbrooke), France Henri (Téluq), Gaby Hsab (UQAM), Philippe Jonnaert (UQAM), Jean-Claude Kalubi (Université de Sherbrooke), Carol Landry (Université Laval), Frédéric Legault (UQAM), Marie-Françoise Legendre (Université de Montréal), Daniel Martin (UQAT), Pierre Mongeau (UQAM), Denise Normand-Guérette (UQAM), Florian Péloquin (Cégep de Lanaudière), Denis Rhéaume (INRS), Jeanne Richer (Cégep de Trois-Rivières), MarieCarmen Rico de Sotelo (UQAM), Magali Robitaille (chercheure en éducation), Anne Roy (chercheure en éducation), Ghislain Samson (Université de Sherbrooke), Lorraine Savoie-Zajc (UQO), Noëlle Sorin (UQTR), Hassane Squalli (Université de Sherbrooke), Carole St-Jarre (chercheure en éducation), Lise St-Pierre (Université de Sherbrooke), Marjolaine St-Pierre (UQAM), Gilles Thibert (UQAM), Michèle Vinet (Université de Sherbrooke), Suzanne Vincent (Université Laval).

\section{Personnes qui ont arbitré des chapitres de cet ouvrage}

Carlos Hernàndez-Blasi (Université Jacques-Premier, Espagne), Jacques Boisvert (Cégep Saint-Jean-sur-Richelieu), Rolande Deslandes (UQTR), Louise Dupuy-Walker (UQAM), Catherine Gosselin (UQAM), Monique L'Hostie (UQAC), Catherine Meyor (UQAM), Jocelyne Morin (UQAM), Michèle Venet (Université de Sherbrooke). 